\title{
Material Geometry the Periodic Table and Chemistry
}

\author{
Markos Georgallides \\ ${ }^{1}$ larnaca (Expelled From Famagusta Town Occupied By The Barbaric Turks Aug-1974), Cyprus \\ Civil-Structural Engineer (Natua), Athens
}

\begin{abstract}
Point in E-Geometry, which is nothing and dimensionless, can be derived from the addition of a Positive (+)and a Negative (-) number while Material point has dimension, the Segment $d s=[\oplus 9$, and originates in the same way. Adding it to numbers i.e. Monads, creates Primary Particles, the Rest-Gravity constituent and the Atoms of Periodic System in Planck's Space-Level. Monads are Spinning because of the inner ElectromagneticWaves E,P which create External Spin and again the Inner Electromagnetic Waves $E, P$ continuing this eternal Cycle. Now is shown the, Why and How the Spinning of Particles and the Way and the Place conserved.

Euclidean-Geometry elucidated the definitions of Geometry-content \{Point, Segment, Straight Line, Plane, Volume Space [S] Anti-space [AS] ,Sub-space [SS] ,Cave, Space-Anti-Space Mechanism of the Six-TriplePoints -Line, that produces and transfers Points of Spaces, Anti-Spaces and Sub-Spaces in a Common Inertial Sub-Space, the Field [MFMF] and Particles] and the same for Material-Geometry which describes the Space and and Energy beyond Plank's length -level [ $G$-Length 3,969.10- 62] , to Point $L v=e i .(N \pi 2) b=10^{-} N=-\infty$ $m \rightarrow 0$.

Energy monads presuppose Energy-Space Base which is the cause of Spaces existence and the motion of particles. This Energy-Space Base, MFMF = Medium-Field of Material-Fragments is the Material-Geometry in the way used in Mechanics and Physics. Property for Doubling Energy in the same Space is encountered in Stationary wave where Energy is proportional to angular velocity, w. Now is shown the How MaterialFragments are built-in E-Geometry and the Why follow its moulds. The Special Problems of E-geometry consist the, Mould Quantization of Euclidean Geometry in it to become $\rightarrow$ Monad, through mould of Space -Anti-space in itself, which is the material dipole in inner monad Structure as is the Electromagnetic cycloidal field. The Unification of Space and Energy becomes through [STPL] Geometrical Mould Mechanism of the minimum Energy-Quanta to Monads $\rightarrow$ Particles, Anti-particles, Bosons, Gravity-Force, Gravity-Field, Photons, Dark Matter, and Dark-Energy, consisting the Material Dipoles in inner monad Structures , with light velocity and continually formulating the ,Zero $\rightarrow$ Discrete ,Particles-Atoms-Elements $\rightarrow$ Infinite Geometrical Universe. Conservation of Energy, as Torsional momentum, is obtained by Dynamical stability of Nucleus-Wheel-Rims.
\end{abstract}

Keywords: Material Geometry, Energy Particles, Periodic Table and Chemistry. MaterialGeometry the Periodic Table and Chemistry.

\section{Periodic motion.}

\section{General}

Harmonic Periodic motion $\mathrm{x}(\mathrm{t})$ is when motion is repeated itself regularly, in equal intervals of time $\mathrm{T}$ (the period of oscillation) and is designated by the time function ,

$x(t)=x(t+T)=x=A \cdot \sin (2 \pi \cdot t / T)=A \cdot \sin \cdot w t=$

$=\mathrm{A} \cdot \cos (\mathrm{kq}-\mathrm{wt})$ because is sinusoidal, where

A is the amplitude of oscillation measured from equilibrium position and for repeated motion $\mathrm{t}=\mathrm{T}$.

Quantity $(2 \pi / T)=w=2 . \pi . f$ is circular frequency,

or $\mathrm{f}=1 / \mathrm{T}=\mathrm{w} / 2 \pi$, is the frequency and, $\mathrm{k}$, is the wave number $\mathrm{k}=2 \pi / \lambda$ and the speed of a wave is $\mathrm{v}=\lambda . \mathrm{f}$ or $\mathrm{w}$ $=\mathrm{v} \cdot \mathrm{k}$ and because of relation of angular velocity $\overline{\mathrm{v}}=\mathrm{w} \cdot \mathrm{r}=\mathrm{w}(1 / \mathrm{k}) \rightarrow \mathrm{w}=\mathrm{v} \cdot \mathrm{k}$ then $\mathrm{r} . \mathrm{k}=1$.

Velocity $\overline{\mathrm{v}}=\dot{\mathrm{x}}=\mathrm{wA} \cdot \cos \mathrm{wt}=\mathrm{wA} \cdot \sin (\mathrm{wt}+\pi / 2)$

$\overline{\mathrm{a}}=\ddot{\mathrm{x}}=\mathrm{w}^{2} \mathrm{~A} \cdot(-\sin w \mathrm{t})=\mathrm{w}^{2} \mathrm{~A} \cdot \sin (\mathrm{w} t+\mathrm{T})$

i.e. Velocity , $\dot{\mathrm{x}}$, and Acceleration $\ddot{\mathrm{x}}$ are also harmonic with the same fre quency of oscillation , and when evaluated lead to the displacement , $\mathrm{x}$, by $\pi / 2$ and $\pi$ radians respectively and the whole system reveals at $\ddot{x}=-$ $\mathrm{w}^{2} \mathrm{~A}$, so that In harmonic motion acceleration to be proportional to the displacement and directed toward the origin , and because also Newton`s second law of motion states that the acceleration is proportional to the force , then harmonic motion can be expected with force varying as kx. (which is Hook`s law F = kx and k, the stiffness coefficient, directed in centrifugal velocity vector $\mathrm{vr}$, on radius $\mathrm{r}$ ) . 
In Free vibration of monads $\mathrm{AB}=\mathrm{q}=[\mathrm{s}+\overline{\mathrm{v}} \square \mathrm{i}]$ and because velocity vector is composed of the centrifugal velocity $\overline{\mathrm{v}} \mathrm{r}$, and the rotational velocity $\overline{\mathrm{v}} \mathrm{q}$, perpendicular to displacement , $\mathrm{x}$, and because viscous damping represented by a dashpot, is described by a force proportional to the velocity as holds $\mathrm{F}=\mathrm{c} \dot{\mathrm{x}}$ where, $\mathrm{c}$, is the damping coefficient , it is a constant of transverse proportionality and this because $\dot{\mathrm{x}} \quad \square \mathrm{dx}$, then it is directional to transverse velocity $\bar{v} \mathrm{y}=\dot{\mathrm{x}} / \mathrm{dt}$ and is holding the

homogenous differential equation $m \ddot{x}+c \dot{x}+k x=0$.

For a flexible string of mass,$\rho$, per unit $\mathrm{dx}$ is stretched under Tension $\mathrm{T}$ and analyzing Newton Laws for tiny length ,dx, then Net Force,$T \ddot{x}=\rho \bar{a}$ and the equation of motion is $\ddot{x}=\left[1 v^{2}\right] \bar{a}$ or $\partial^{2} \mathrm{y} \partial \mathrm{x}^{2}=\left[1 \mathrm{v}^{2}\right] . \partial^{2} \mathrm{y} \partial \mathrm{t}^{2} \ldots .(1)$, where $\mathrm{v}=\sqrt{ } \mathrm{T} \rho=\sqrt{ } \mathrm{Tm}$

The general solution of (1) is $\mathrm{y}=\mathrm{F} 1(\mathrm{ct}-\mathrm{x})+\mathrm{F} 2(\mathrm{ct}-\mathrm{x})$ where $\mathrm{F} 1, \mathrm{~F} 2$ are arbitrary functions and regardless of the type of function $\mathrm{F}$, the argument $(\mathrm{ct} \pm \mathrm{x})$ upon differentiation leads to the equation,

$\partial^{2} \mathrm{~F} \partial \mathrm{x}^{2}=\left[1 \mathrm{v}^{2}\right] . \partial^{2} \mathrm{~F} \partial \mathrm{t}^{2} \ldots .(2)$, where $\mathrm{F}=$ The tension,

$\mathrm{v}=$ the velocity of wave propagation Another general solution of (1) is that of separation of variables as $\mathrm{y}(\mathrm{x}, \mathrm{t})=\mathrm{Y}(\mathrm{x}) \cdot \mathrm{G}(\mathrm{t})$

where then (1) becomes $\rightarrow 1 \mathrm{Y} \partial^{2} \mathrm{Y} \partial \mathrm{x}^{2}=\left[1 \mathrm{v}^{2}\right] 1 \mathrm{G} . \partial^{2} \mathrm{G} \partial \mathrm{t}^{2}$ and because of independent variables $\mathrm{x}, \mathrm{t}$ are both constant the general solutions are, $\mathrm{Y}=\mathrm{A} \operatorname{sinwv} \mathrm{x}+\mathrm{B} \cos w \mathrm{v} \mathrm{x}=\mathrm{A} \sin \mathrm{wt}+\mathrm{D} \cos \mathrm{wt}$, where arbitrary constants A,B,C,D depend on boundary conditions and for $y(0, t)=0$ will require $B=0$ and for $y(1, t)=0$ the solution lead to equations, $\mathrm{y}=[\mathrm{C} \sin \mathrm{wt}+\mathrm{D} \cos \mathrm{wt}] \sin \mathrm{wv} \mathrm{x}$ and $\sin w \mathrm{wlv}=0$ or $\mathrm{Wn} .1 \mathrm{v}=2 \pi .1 \lambda=\mathrm{n} . \pi$ where

$\mathrm{n}=1,2,3, . \mathrm{n} \lambda=\mathrm{vf}$ the wavelength, $\mathrm{fn}=\mathrm{n} 21 \mathrm{v}=\mathrm{n} 21 \sqrt{\mathrm{T} \rho}$ is frequency and $\mathrm{Y}=\mathrm{sin} \mathrm{n} \pi \mathrm{xl}$ In case of vibration initiated in any manner $\mathrm{y}(\mathrm{x}, \mathrm{t})=\Sigma[\mathrm{Cn} \cdot \operatorname{sinWn} . \mathrm{t}+\mathrm{Dn} \cdot \cos \mathrm{Wn} \cdot \mathrm{t}] \cdot \sin (\mathrm{n} \pi \mathrm{x} / \mathrm{l})^{\infty} \mathrm{n}=1$

Example 1:

To determine the $\mathrm{Cn}$ and $\mathrm{Dn}$ of above equation . solution : At $\mathrm{t}=0$ the displacement and velocity are , $\mathrm{y}(\mathrm{x}, 0)=\Sigma \mathrm{Dn} \cdot \sin (\mathrm{n} \pi \mathrm{x} / 1)^{\infty} \mathrm{n}=1$ and velocity $\dot{\mathrm{y}}(\mathrm{x}, 0)=\Sigma \mathrm{Wn} \cdot \mathrm{Cn} \cdot \sin (\mathrm{n} \pi \mathrm{x} / 1)^{\infty} \mathrm{n}=1=0$ and both multiplying by $\sin (\mathrm{k} \pi \mathrm{x} / \mathrm{l})$ and integrating from $\mathrm{x}=0$ to $\mathrm{x}=1$, all terms become zero except $\mathrm{n}=\mathrm{k}$ and $\mathrm{Dk}=2 l \int_{\mathrm{y}}(\mathrm{x}, 0) \cdot \sin (\mathrm{k} \pi \mathrm{x} \mathrm{l}) \mathrm{dx} \mathrm{l} 0$ and $\mathrm{Ck}=0 \mathrm{k}=1,2,3$.

For standing waves equation of motion is the sum of a right moving and a left moving wave, or Ysta = A.sin. $(k n x+w n t)+A \cdot \sin \cdot(k n x+w n t)=[2 A \cdot \sin (k n x) \cdot \cos (w n t)$ where $k n=2 \pi / \lambda n$ and, $\sin (k x) \rightarrow$ is the Spatial dependence at locations $\mathrm{x}=0, \lambda / 2, \lambda, 3 \lambda / 2, \mathrm{n} \lambda \mathrm{n} 2$ called the nodes . Material Geometry the Periodic System and Chemistry . 3 At nodes amplitude ,A, is always zero and at locations $\mathrm{x}=\lambda / 4,3 \lambda / 4,5 / 4$ called the Anti-nodes where the amplitude becomes maximum $\cos (\mathbf{w t}) \rightarrow$ is the Time Oscillation dependence where corresponding frequency $\mathrm{f} 1=\mathrm{v} / 2 \mathrm{x}=\mathrm{v} / \mathrm{n} . \lambda \mathrm{n}$ and $\mathrm{fn}=\mathrm{n} . \mathrm{f} 1$, i.e. $\mathbf{n}$, more times Energy is stored in fn frequency, showing the way of Energy quantization in constant caves [49] .

Since energy $E=h . f$ then frequency $f=E / h$. Angular velocity $w=2 \pi / T=2 \pi . f=2 \pi .(E / h)=E /[$ h/2T] , or $\mathbf{w}=\mathbf{E} /(\mathbf{h} / \mathbf{2} \pi), \mathbf{E}=\mathbf{w} \cdot[\mathbf{h} / \mathbf{2} \pi]$ i.e

Proportional to energy $\mathrm{E}$, since $\mathrm{h} / 2 \mathrm{~T}$ is constant.

Energy in a standing wave : Kinetic energy is $E K=d K d x(x, t)=m v^{2} / 2==m 2 \partial y^{2} \partial t^{2}=m 2 A^{2} \cdot w^{2}$ ..(1)

Potential energy is EU $=d K d x(x, t)=[F / 2] \partial y^{2} \partial t^{2}$ and Power transmitted $P(x, t)=-F \partial y \partial x \partial y \partial t$ Total Energy $\mathrm{ET}=\mathrm{EK}+\mathrm{EU}=[\mathrm{m} 2] \partial \mathrm{y}^{2} \partial \mathrm{t}^{2}+[\mathrm{F} 2] \partial \mathrm{y}^{2} \partial \mathrm{t}^{2}$

In Sinusoidal waves $\mathrm{dKdx}=\mathrm{dUdx}=[\mathrm{m} 4] \partial \mathrm{y}^{2} \partial \mathrm{t}^{2}=\mathrm{m} 4 \mathrm{~A}^{2} \mathrm{w}^{2}$

Torsional Vibrations of Rods is similar to that of of longitudinal vibration of rods and for the angle of twist in any length ,dx, due to Torque $T$ is $d \theta$ then $d \theta=T d x / I p . G$ where , Ip.G is the torsional stiffness as the product of the Polar-moment, Ip , of Inertia of the cross-section area , and the shear modulus ,G, of elasticity , and which net Torque is $\rightarrow \partial \mathrm{T} \partial \mathrm{x} d \mathrm{x}=\mathrm{IpG} \partial^{2} \theta \partial \mathrm{x}^{2} \mathrm{dx}$. The differential equation of motion becomes by equating torque to the product of the mass moment of inertia $\rho I p d x$ of the element and the angular acceleration $\partial^{2} \theta \partial t^{2}$ in it and is , $\rho \mathrm{Ipdx} \partial^{2} \theta \partial \mathrm{t}^{2}=\mathrm{IpG} \partial^{2} \theta \partial \mathrm{x}^{2} \mathrm{dx}$ and $\partial^{2} \theta \partial \mathrm{t}^{2}=\mathrm{G} \rho . \partial^{2} \theta \partial \mathrm{x}^{2} \ldots(2)$ where $\theta \equiv$ replace $\mathrm{u}, \mathrm{G} \rho \equiv \mathrm{E} \rho$ of longitudinal vibration with the general solution given for $\theta$ is ...(3)

$\theta=[A \sin w \sqrt{ } \rho G \mathrm{x}+\mathrm{B} \cos w \sqrt{ } \rho \mathrm{G} x] .[\mathrm{C} \sin w t+\mathrm{D} \cos w \mathrm{t}]$

\section{Example 2:}

To find the equation of the natural frequencies of a uniform rod in torsional oscillation with one end Fix and the other end Free. The solution :

The boundary conditions are, a.. when $\mathrm{x}=0$ then $\theta=0$ resulting to $\mathrm{B}=0 \mathbf{b}$.. when $\mathrm{x}=1$ then Torque $=$ 0 or $\partial \theta / \partial \mathrm{x}=0$ and resulting to $\cos w \sqrt{ } \rho / G l=0$ and which is satisfied with the following angles, $\mathbf{w n} \sqrt{ } \sqrt{ } \mathbf{G l}=\pi / 2$, $3 \pi / 2,5 \pi / 2 \ldots .(n+1 / 2)$. $\pi$ and the natural frequency of the rod of length 1 , is $\mathbf{w n}=(n+1 / 2) . \pi l \sqrt{ } \mathrm{G} \rho$ where $n$ $=0,1,2,3, \ldots$

where $\mathrm{m}=\rightarrow$ is the mass of the rod 
$\rho=\rightarrow$ is the density per unit length $d x$,

$\mathrm{G}=$ The shear modulus of the rod,

and for Electromagnetism

$\varepsilon=$ The permittivity of the free space , Dielectric constant multiplier .

$\mu=$ The Permeability of transverse space ,

Dielectric constant multiplier.

The Total ET , Kinetic EK, and Potential EU , energy per length is their sum as follows ,

Total Energy $\mathrm{ET}=\mathrm{EK}+\mathrm{EU}=[\mathrm{m} 2] \partial \mathrm{y}^{2} \partial \mathrm{t}^{2}+[\mathrm{T} 2] \partial \mathrm{y}^{2} \partial \mathrm{t}^{2}$ The work done per cycle is $\mathrm{W}=\pi$.v.A sin $\mathrm{wt}$ where $\mathrm{A}=$ Initial amplitude at $\mathrm{x}=0$.

\section{Introduction}

Point, which is nothing and has not any Position may be anywhere in Space, therefore, the unique Primary point A, being nothing also in no Space, is the only Point and nowhere, i.e. Primary Point is the only Space and from this all the others which have Position, therefore it is the only Space and so to exist point $\mathrm{A}$, at a second point B somewhere else, point A must move towardspoint B , where then A $\equiv$ B.Point B is the Primary Anti-Space which Equilibrium point $\mathrm{A}$, It is $[\mathrm{PNS}]=[\mathrm{A} \equiv \mathrm{B}]$. The position of points in $[\mathrm{PNS}]$ creates the infinite dipole and all quantum quantities which acquire Potential difference and an Intrinsic moment $\pm \Lambda$ in the three Spatial dimensions $(x, y, z)$ and on the infinite points of the ( i ) Layers at these points, which exist from the other Layers of Primary Space, Anti-Space and Sub-Space , and this is because Spaces = monads = quaternion [9]. Since Primary point A, is the only Space then on this point exists the Principle of Virtual Displacements as , The Material Geometry and the Origin of Energy-Particles in Periodic System $4 \mathrm{~W}=$ $\int \mathrm{P} . \mathrm{dsBA}=0$ or $[\mathrm{ds} .(\mathrm{PA}+\mathrm{PB})=0]$, i.e. for any $\mathrm{ds}=$ vector $>0$, Impulse $\mathrm{P}=(\mathrm{PA}+\mathrm{PB})=0$ and $[$ ds. $(\mathrm{PA}+\mathrm{PB})=$ $0]$, Therefore, Each Unit $A B=d s>0$, exists by this Inner Impulse $(P)$ where $(P A+P B)=0$ i.e.

The Position and Dimension of all Points which are connected across the Universe and that of Spaces exists because of this Static equilibrium and Inner Impulse, on the contrary should be one point only ( Primary Point $\mathrm{A}=$ Black Hole $\rightarrow \mathrm{ds}=0$ and $\mathrm{P}=\infty)$, as the repellency of $\mathrm{A}, \mathrm{B}$ points by (PA) , ( PB) opposite forces as $\leftrightarrow$ dipole $\rightarrow[\{\mathrm{A}(\mathrm{PA}) \leftarrow 0 \rightarrow((\mathrm{PB}) \mathrm{B}\}] . \leftarrow[17,23]$.

All points may exist with $\mathrm{P}=0 \rightarrow(\mathrm{PNS})$ and also with $\mathrm{P} \neq 0 \rightarrow($ Spaces $),(\mathrm{PA}+\mathrm{PB}=0)$, for all points in Spaces and Anti -Spaces, therefore [PNS] is self -created, and because at each point may exist also with $P \neq 0$, then [ PNS ] is a ( perfectly Homogenous, Isotropic and Elastic Medium , in spatial and Temporal domain) Field with infinite points which have $\mathrm{a} \pm$ Charge with $\mathrm{P}=0 \rightarrow \mathrm{P}=\Lambda \rightarrow \infty$ and work ( W) is quantized on material points as EM wave and as spin $\pm(\overline{\mathrm{p}})$ and from this equilibrium quantized angular momentum $\Lambda$, independently of time , is capable of forming the Wave nature of Spaces motion , following the Boolean logic and distorting momentum $\bar{p}=\Lambda$, as energy, on the intrinsic orientation position of material points, on all points of the microscopic and macroscopic homogeneity , and since also in common circle both rotational velocity, $\overline{\mathrm{w}}$, and momentum, $\bar{\Lambda}$, are constants , and thus consist a Pure quaternion, which is the cause of their Inner, motion as The Electromagnetic wave which also produces Spin , and of their Outer Spin, The screw helically Kinetic Energy , causing the Outer wave Motion , so conjugation of the two is , $(\partial / \partial \mathrm{t}, \overline{\mathrm{w}}) \odot(0, \Lambda)=$

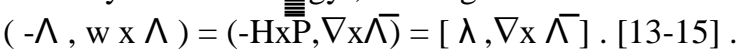

Since points A, B of [PNS] coincide with the infinite Points, of the infinite Spaces, Anti-Spaces and Sub-Spaces of [PNS] and exists rotational energy $\pm \Lambda$ and since Motion may occur at all Bounded Sub -Spaces $( \pm \Lambda, \lambda)$, then this Relative motion is happening between all points belonging to [PNS] and to those points belonging to the other Sub-Spaces (A $\equiv$ B). The Infinite points in [PNS] form infinite Units (monads) AiBi $=\mathrm{d} \overline{\mathrm{s}}$ , which equilibrium by the Primary Anti-Space by an Inner Impulse (P) at edges A, B where P i A + P i B $\neq 0$, and $\mathrm{ds}=0 \rightarrow \mathrm{N} \rightarrow \infty$. Monad, (Unit) $\mathrm{A} \mathrm{B}$, is the ENTITY and $[\mathrm{A}, \mathrm{B}-\mathrm{PA}, \mathrm{P} \overline{\mathrm{B}}]$ is the LAW, so Entities are embodied with the Laws.

Entity is quaternion $\bar{A} B$, and law $|A B|=$ length = the Real part which is Space of points $A, B$ and imaginary part forces , $\mathrm{PA}, \mathrm{P} \overline{\mathrm{B}}$ or fields of $\mathrm{AB}$

The Inner impulse, inward Work W, of any two points A, B of distance ds =AB , is Electrically parallelized to Electric-Field E, inside a Sphere of radius $r$, and Charge $\mathrm{Q}$, equal to work $\mathrm{W}$, in distance ds .

From work $\mathrm{W}=\mathrm{P} . \mathrm{ds}=($ Work in cave $\mathrm{r}) /($ Work in cave $\mathrm{ds})=\mathrm{W}=\mathrm{Wr} \mathrm{r}^{3} \mathrm{ds}^{3}=\mathrm{Qrr}^{3} \mathrm{ds}^{3}$

and force $\mathrm{P}$ is equal to the Electric field intensity,

$\mathrm{Er}=\mathrm{Q} \cdot \mathrm{r} 4 \pi \varepsilon \cdot \mathrm{ds}^{3}=\mathrm{P}$

\section{Remarks :}

Applying above equations for Initial Space-point $\mathrm{A}$, to Anti-Space point $\mathrm{B}$ spherically, then work $\mathrm{W}=$ P.ds and for $\mathrm{ds}=0$ and $\mathrm{W}=0$ then force $\mathrm{P}$ is any constant or infinite, $\infty$. For $\mathrm{ds}=0$ and $\mathrm{W}=\mathrm{Q}=0$ then $\mathrm{Q}$. $\mathrm{r} 4 \pi \varepsilon \cdot \mathrm{ds}^{3}=0 . \mathrm{r} 4 \pi \varepsilon .0^{3}$ is also any constant or infinite, $\infty$ as above . 
Since Radial fields are drawn from a center point, Electric fields are drawn pointing from positive to negative like the flow of current and as like the magnetic and gravitational fields .

Since $\mathrm{W}=\int \mathrm{P} . \mathrm{dsBA}=\int$ Er.dsBA , then work is equal to the Electric-Flux, and since Electric-Flux is the rate of flow of electric field through area of a sphere so thus, Electric-field of this unique and Initial dipole $A \leftrightarrow B$ is created. i.e.

All Space and Anti-Space which is universe, is a Giant and Uniform Electric field, with Lines directed from $\mathbf{A} \equiv \bigoplus$ to $\ominus \equiv \mathbf{B}$ meaning that Work as Energy ( i.e. the spherical displacement ds ), is stored in this huge cave $\mathrm{AB} \rightarrow \infty$.

It was shown [49] that ,The Infinite Electric dipole $N N \equiv[\bigoplus \leftrightarrow \ominus]$ in this Homogeneous and uniform Electricfield $[\mathrm{W}=\mathrm{Q}]$ experience no net force but experience a net Torque . Furthermore Dipole NN possess intrinsic Maxwell`s Displacement current forming the outer Spin as Torque which moves it.

Generally Quantization of E-geometry happens in any Layer, Level, and between the Layers, in the same way as Electricity flows in the two ways, which is either in a Direct Current [DC], or in an Alternating [AC] Current. Quantization of E-geometry, is nothing but the Position of points at Extrema, either forming the multiplicity of a Layer of, (Points, lines, Planes, Material Geometry the Periodic System and Chemistry .

5 Volumes ), or Material Geometry forming the multiplicity of a material Layer of ,( Points , lines , Planes , Volumes ) discrete elements .

A wide analysis for monads and EM-Waves in [49]

\section{Definition Of Quantization}

\subsection{Quantization .}

Quantization is the concept ( the Process ) that any, Physical Quantity $\rightarrow$ [PQ] of the objective reality ( Matter, Energy or Both ) is mapping the Continuous Analogous, the points, to only certain Discrete values . Quantization of Energy is done in Space-tanks , on the material points, tiny volumes and on points consisting the Equilibrium, all the Opposite

Twin, of Space Anti-space.

In Geometry [PQ] are the Points, nothing, only, transformed into Segments, Lines, Surfaces, Volumes and to any other Coordinate System such as $(\mathrm{x}, \mathrm{y}, \mathrm{z}),(\mathrm{i}, \mathrm{j}, \mathrm{k})$ and which are all quantized .

Quantization of E-geometry is the way of Points to become as $\rightarrow$ ( Segments, Anti-segments $=$ Monads $=$ Anti-monads), (Segments , Parallel-segments = Equal monads), ( Equal Segments and Perpendicular-segments $=$ Plane Vectors $),($ Un-equal Segments twice - Perpendicular - segments $=$ The Space Vectors $=$ Quaternion $)$ .[46]

In Philosophy [PQ] are the concepts of Matter and of Spirit or Materialism and Idealism .

a).. Anaximander, claimed that non of the elements could be, Arche and proposed, apeiron , an infinitive substance from which all things are born and to which all will return.

b).. Archimedes, is very clear regarding the definitions, that they say nothing as to whether the things defined exist or not, but they only require to be understood. Existence is only postulated in the case where [PQ] are the Points to Segments (magnitudes = quantization process). In geometry we assume Point, Segment, Line , Surface and Volume, without proving their existence, and the existence of everything else has to be proved .

The Euclid`s similar figures correspond to Eudoxus` theory of proportion .

c).. Zenon, claimed that, Belief in the existence of many things rather than, only one thing, leads to absurd conclusions and for, Point and all its constituents will be continuous without magnitude. Considering Points in space are a distinct place even if there are an infinity of points, defines the Presented in [44] idea of Material Point .

d).. Materialism or and Physicalism, is a form of philosophical monism and holds that matter ( without defining what this substance is ) is the fundamental substance in nature and that all phenomena, including mental phenomes and consciousness, are identical with material interactions by incorporating notions of Physics such as space-time, physical energies and forces, dark matter and so on .

e).. Idealism , such as those of Hegel, ipso facto , is an argument against materialism ( the mind-independent properties can in turn be reduced to the subjective percepts ) as such the existence of matter can only be assumed from the apparent ( perceived ) stability of perceptions with no evidence in direct experience .

Matter and Energy are necessary to explain the physical world but incapable of explaining mind and so results , dualism. The Reason determined in itself and its relation to the world creates the very old question as, what is the ultimate purpose of the world?.

f).. Hegel's conceive for mind, the Idea, defines that, mind is Arche and it is retuned to [PQ] the subjective percepts, while Materialism holds just the opposite . 
In Physics [PQ] are The, Electrical charges, Energy, Light, Angular momentum, Matter which are all quantized on the microscopic level. They do not seem quantized in the macroscopic scale because the size of the steps between each possible value is so small .

a).. De Broglie found that, light and matter at subatomic level display characteristics of both waves and particles which move at specific speeds called Energy-levels .

b).. Max Planck found that, Energy and frequency of the Electromagnetic radiation is quantized as the relation $\mathrm{E}=\mathrm{h} . \mathrm{f}$ where $\mathrm{h}$ is a constant and frequency only defines the Physical Quantity of microcosmic level .

In Mechanics, Kinematics describes the motion while Dynamics causes the motion.

c).. Bohr model for Electrons in free-Atoms is the Scaled Energy levels, for Standing-Waves is the constancy of Angular momentum, for Centripetal-Force in electron orbit, is the constancy of Electric Potential, for the Electron orbit radii , is the Energy level structure with the Associated electron wavelengths.

d).. Hesiod Hypothesis [PQ] is Chaos, i.e. the Primary Point from which is quantized to Primary Anti-Point . [ From Chaos came forth Erebus, the Space Anti-space, and Black Night, The [STPL] The Material Geometry and the Origin of Energy-Particles in Periodic System

Mechanism, but of Night were born Aether, The rest Gravity dipole Field connected by the Gravity Force , and Day , Particles Anti-particles, whom she conceived and Bare, The Equilibrium between Particles Anti-particles, in Spaces Anti-spaces , from union in love with Erebus ] . [43-48] e).. The current method for Physical Quantity

[PQ] is the Material Geometry where Points are the non-collided elements in Caves and are the two Positive and Negative breakages $\pm s^{2}=(\mathrm{wr})^{2}$ and the Vector breakage [ $\left.\square \mathrm{i}\right]=2 \mathrm{~s}^{2}=2$. $(\mathrm{wr})^{2}$.[22] With these three elements is possible by following the Geometrical Rules (and those of Algebra, as Commutation, Distribution, Expansion , Association, Absorbation, Multiplication ) and Permutation and Repetition to build all Physical World as shown below.

Point in E-geometry is considered nothing without Position and direction while in Material Geometry Point are both massive Units $+s^{2}$ and $-s^{2}$ and for Two Units ,Line-Sector is the Dipole $\left[s^{2} \leftrightarrow-s^{2}\right]$. Infinite Series of $\left[s^{2} s^{2} s^{2} s^{2} \ldots\right],\left[-s^{2}-s^{2}-s^{2}-s^{2} \ldots\right]$, consist the Material points in Lines .

Three Units of $\left[\mathrm{s}^{2} \mathrm{~s}^{2} \mathrm{~s}^{2}\right],\left[-\mathrm{s}^{2}-\mathrm{s}^{2}-\mathrm{s}^{2}\right]$ not coinciding consist the Material Plane of the Infinite Lines . Four Units of $\left[s^{2} s^{2} s^{2} s^{2}\right],\left[-s^{2}-s^{2}-s^{2}-s^{2}\right]$ not coinciding consist the Material Volume of Infinite Planes.

Five Units of $\left[s^{2} s^{2} s^{2} s^{2} s^{2}\right],\left[-s^{2}-s^{2}-s^{2}-s^{2}-s^{2}\right]$ not coinciding consist the Material Fifth -Space -Volume of Infinite Planes.

$\mathrm{N}$ Units of $\left[\mathrm{s}^{2} \mathrm{~s}^{2} \mathrm{~s}^{2} . . \mathrm{N}\right],\left[-\mathrm{s}^{2}-\mathrm{s}^{2}-\mathrm{s}^{2} . . \mathrm{N}\right]$ not coinciding consist the Material N-Space-Volume of Infinite Planes. Since Material-geometry follows E-geometry so , all laws of nature are also those of Geometry .i.e.

From a Set of infinite rest Units choosing two of them, is found Mendeleyev Periodic table in Planck`s level 10-35m ( this Property issues in all geometrical caves and in a cave of 10-62m which is Gravity level in which Gravity-Field exists) and also all models of the atom as follows , 1.. Moulds $\rightarrow$ The minimum Number of Points in each Level is [ 1 for Point , 2 for Line-sector , 3 for Plane , 4 for Volume ,5.. $\mathrm{m}$ for m Spaces. 2.. Units $\rightarrow$ The maximum number of Units in a Point is $2,[1$ Positive $\bigoplus$ and 1 Negative $\Theta]$

The possible Repetitive Permutations for moulds and Units are MouldUnits $=\mathrm{m}^{2}$, for every mould so the Available Extrema Positions for each Point, Line -sector ,Plane, Volume, $\mathrm{m}$ Space are as, $\mathrm{m}=1 \rightarrow 1^{2}=1 \mathrm{x} 2$ times $=2 \rightarrow$ for Point

$\mathrm{m}=2 \rightarrow 2^{2}=4 \times 2$ times $=8 \rightarrow$ Line-vector

$\mathrm{m}=3 \rightarrow 3^{2}=9 \times 2$ times $=18 \rightarrow$ Plane

$\mathrm{m}=4 \rightarrow 4^{2}=16 \times 2$ times $=32 \rightarrow$ Volume

$\mathrm{m}=5 \rightarrow 5^{2}=25 \times 2$ times $=50 \rightarrow$ Volume

$\mathrm{m}=\mathrm{n} \rightarrow \mathrm{m}^{2}=\mathrm{m} . \mathrm{m} \times 2$ times $=2 . \mathrm{m}^{2} \rightarrow \mathrm{m}$ Space and which are all based on Geometric logic only, agreeing with objective reality.

\subsection{Extremes Principle or Extrema :}

All Principles are holding on any Point A. For two points A, B not coinciding, exists Principle of Inequality which consists another quality. Any two points exist in their Position under one Principle, Equality of Stability, (Virtual displacement which presupposes Work in a Restrain System ).[16-17-44].

This Equilibrium presupposes homogenous Space and Symmetrical Anti-Space .

For two points A, B which coincide, exists the Principle of Superposition which is a Steady State containing Extrema for each point separately.

Extrema, for a point $\mathrm{A}$ is the Point, for a straight line the infinite points on opposite line, either these coincide or not or these are in infinite, and for a Plane the opposite infinite lines and points with all combinations and Symmetrical ones, i.e. all Properties of Euclidean geometry levels, compactly exist in Extreme opposite, Points, Lines, Planes, circles by following anode or descend sequence .The same also in Mechanics (Central motion) and between, Linear and Angular Momentum, as the < Spin of particles $>$. 
Since Extreme is holding on Points, lines, Surfaces, Volumes, bodies etc., therefore all their compact Properties(Principles of Equality, Arithmetic and Scalar, Geometric Segments and Vectors , Proportionality, Qualitative, Quantities, Inequality, Perspectivity etc.), exist also in the common opposite context magnitude to direction, therefore in Superposition the magnitude $\mathrm{AB}$ is equal and constant in both directions, or any other direction $\neq 0$, as $[|\mathrm{A}, \mathrm{B}|-\mathrm{P} \overline{\mathrm{A}}, \mathrm{P} \overline{\mathrm{B}}]$ i.e.

Any Segment $\mathbf{A B}$ between two points $\mathrm{A}, \mathrm{B}$ consist a Vector described by the magnitude, $\mathrm{AB}$, and directions $\tilde{\mathrm{A}} \mathrm{B}$ , B $\tilde{A}$ and in case of Superposition $\bar{A} A, A \bar{A}$, where Properties of Vectors, Proportionality, Symmetry, etc. exist either on edges $\mathrm{A}, \mathrm{B}$ or on segment $\mathrm{AB}$ as $\rightarrow$

A quantity to Anti-quantity, a monad to Anti-monad, and since it is either a scalar or a vector and by their distinct definitions are, Material Geometry the Periodic System and Chemistry . Scalars, are quantities that are fully described by a magnitude or numerical value alone in Anti-Scalars. Energy which is motion to Anti-motion, i.e. to the Anti-trajectory.

According to Thales theorem, Figure.2-3 if two intersecting lines PA, PB are intercepted by a pair of Parallels $\mathrm{AB} / / \mathrm{A}^{\prime} \mathrm{B}^{\prime}$, then ratios $\mathrm{PA} / \mathrm{AA}^{\prime}, \mathrm{PB} / \mathrm{BB}^{\prime}, \mathrm{PA} / \mathrm{PA}^{\prime}, \mathrm{PB} / \mathrm{PB}^{\prime}$ of lines, or ratios in similar triangles $\mathrm{PAB}$ , $\mathrm{PA}^{\prime} \mathrm{B}^{\prime}$ are equal, or ratio $\lambda=\left[\mathrm{PA} / \mathrm{AA}^{\prime}\right]=\left[\mathrm{PB} / \mathrm{BB}^{\prime}\right]$. In case line $\mathrm{A}^{\prime} \mathrm{B}^{\prime}$ coincides with $\mathrm{AB}$, then $\mathrm{AA}^{\prime}=\mathrm{AA}^{\prime}$ , $\mathrm{BB}^{\prime}=\mathrm{BB}$, i.e. exist Extreme and then $\lambda=[\mathrm{PA} / \mathrm{AA}]=[\mathrm{PB} / \mathrm{BB}],($ Principle of Superposition ), where property of scalar exists on common segment $\mathrm{AB}$.

Vectors are Imaginary quantities that are fully described by a constant magnitude and, change direction in order to move, or to keep their constant numerical value to Anti -Space.

Strain $(\varepsilon)=$ change of length / length $\rightarrow$ It is the relative change in shape or size of an object due to externallyapplied forces . Young modulus $(E)=$ tensile stress $/$ tensile Strain. Stress $(\sigma)=$ E. Strain $=$ E. $\varepsilon$, Strain $=$ Stress $/$ $\mathrm{E}=\varepsilon=\varepsilon(\mathrm{u}, \mathrm{v}, \mathrm{w})$, for the three dimensions $. \mathrm{G}=$ shear modulus $=\mathrm{E} \cdot \mathrm{m} 2(\mathrm{~m}+1)$ where $\mathrm{m}=$ Poisson`s ratio $=1 / \mu=$ $10 / 3 \cdot[26-27]$

Volume and, Surface, Plane stress :

A material is said to be under Plane stress if the stress-vector is zero across a particular surface, i.e. $\sigma 3$ $=0$ or in stress cube,$\sigma z=T y z=T X z=0$ From mathematical theory of Elasticity a surface, $\mathbf{F}$, under pressure, $\mathbf{p}$, due to a transverse force , $\mathbf{P}$, is $\mathrm{p}=\mathrm{P} / \mathrm{F}$ pervaded in all, and around surface and if force direction forms an angle $\theta$ , then Principal stresses $\boldsymbol{\sigma} 1, \boldsymbol{\sigma} 2$ and Shear stresses $\mathrm{T} 1,2$ are as , $\sigma 1,2=(\sigma 1+\sigma 2) / 2 \pm(1 / 2) \cdot \sqrt{ }(\sigma 1-\sigma 2)^{2}+4 .(\mathrm{T} 1,2)^{2}$ and $\rightarrow \tan (2 \theta)=2 . \mathrm{T} 1,2 /(\sigma 1-\sigma 2)$

When surface becomes a point [ This is Extreme case where surface is interchanged as line or linesegment, it is the same as the infinite small ,ds, in Calculus ], then $\sigma 2=0$ and $T 1,2$ very small .

Since force $\mathrm{P}$ is a vector, then as in cross-product to a right-handled coordinate system where exists $\sigma 2=0$ and $\mathrm{T} 1,2=\sigma 1$, equation (a) becomes

$\sigma 1,2=\sigma 1 / 2 \pm(1 / 2) \cdot \sqrt{ } \sigma 1^{2}+4 \cdot \sigma 1^{2}=$

$\sigma 1,2=\sigma 1 / 2[1 \pm(\sqrt{ } 5)]$

i.e. Stress $\sigma$ on a point is manifested as $\sigma=\mathbf{P} / \mathbf{d F}$ and as $\mathbf{d F}=\mathbf{0} \rightarrow \overline{\boldsymbol{\sigma}}=\mathbf{P} /[\mathbf{d F} \rightarrow \mathbf{0}]$ becomes momentum $\mathbf{M} \cdot \overline{\mathrm{V}}$ , and this because energy $\mathrm{mv}^{2} / 2$ as linear motion becomes only rotational energy.

Since Stationary force $\mathrm{P}$ exists independently of the acting area then for zero surface (a point) stresses $\mathrm{P} / \mathrm{F}$ vanish, and Stationary force $\mathbf{P}$ becomes a Moving force $\overline{\mathbf{P}}$ and exists as momentum $\mathbf{m v}$ with $\mathrm{m}=\mathrm{M}=1$ (the Extreme hypothetical Reaction to the motion) i.e. the velocity $\overline{\mathbf{v}}$ at this point and which is decomposed in the two perpendicular velocities $\overline{\mathrm{v}} 1, \overline{\mathrm{v}} 2$, where then equation (b) is transformed as ,

$\sigma \mathbf{1}=\overline{\mathbf{v} 1}=(\sigma 1) / \mathbf{2}(\mathbf{1}+\sqrt{ } \mathbf{5})$ and $\sigma \mathbf{2}=\overline{\mathbf{v}}=(\sigma 2) / \mathbf{2}(\mathbf{1}-\sqrt{\mathbf{5}}) .$. (c) and Total stress $\boldsymbol{\sigma}=\mathbf{P} / \mathbf{d F}=\mathbf{0} \rightarrow \overline{\mathbf{P}}=\mathbf{m} \cdot \overline{\mathbf{a}} \rightarrow \overline{\mathbf{v}}$ $=\{\overline{\mathbf{v}} \perp \overline{\mathbf{v} 2}\}=\{\overline{\boldsymbol{\sigma} 1} \perp \overline{\boldsymbol{\sigma} 2}\}=$ Constant , where,$\overline{\mathbf{v} 1} \rightarrow$ represents the Inward compressible radial velocity and $\overline{\mathbf{v} 2}=\overline{\mathbf{v}} \rightarrow$ represents the Transverse Outward stretchable radial velocity of point, which is transformed into , $\boldsymbol{\sigma} \mathbf{1} \rightarrow$ representing the Inward compressible radial pressure, $\boldsymbol{\sigma} \mathbf{2}=\boldsymbol{\sigma} \mathbf{1} \rightarrow$ representing the Transverse Outward stretchable radial pressure of material point, $\mathbf{m}=$ the reaction to the change of distance , to the velocity motion (the mass ), $\mathbf{a}=$ the change of velocity motion ( the acceleration definition ), i.e. Force $\mathbf{P}$, in a Material body appears as Kinetic energy,$\rightarrow$ in an Elastic surface is appearing as Principal and Shear stress,$\rightarrow$ in a Material line or Segment as tension,$\rightarrow$ in Euclid line becomes velocity on line or , a Free Velocity moving Line-Segment , or a moving Vector (quaternion = monad ). The minimum Quantized Energy , Quanta $=2 s^{2}$, is diffused through the minimum Quantized Space, Quanta , $\mathrm{s}^{2}$, in all quantized spaces, which are Particles, [MFMF] Field, moving Vectors, free velocity monads, Material lines $\rightarrow$ Surfaces and solid Bodies. Since also it is a moving energy then diffusion (decomposition) of the stored energy follows Pythagoras theorem in a New Configuration with Scalar and Vector magnitudes such that satisfy the principle of conservation of linear momentum .

Points in Space carry A priori the work $\mathrm{W}=\int \mathrm{A}-\mathrm{B}[\mathrm{P} . \mathrm{ds}]=0$, where magnitudes $\mathrm{P}, \mathrm{ds}$ can be varied The Material Geometry and the Origin of Energy-Particles in Periodic System 8 leaving work unaltered. Using the work formulas of elasticity then In and On Surface work W is, $\mathrm{W}=\left[\left(\sigma 1^{2}+\sigma 2^{2}\right) / 2-(\sigma 1 . \sigma 2) / \mathrm{m}\right] / \mathrm{EF} \mathrm{Wv}=$ 
$\left[\left(\sigma 1^{2}+\sigma 2^{2}-\sigma 1 . \sigma 2\right) / 6 . G F\right]$ where $, \sigma 1, \sigma 2$, Are the Principle stresses , W , Is the work Inward radial surface , $\mathrm{Wv}$, Is the work Onward radial surface (the transverse). Placing equation (c) in above work equations then Work as energy becomes , $\mathrm{W}=\left[\mathrm{P}^{2} / 4 \cdot \mathrm{EF}^{2}\right] .(6+4 / \mathrm{m}), \mathrm{Wv}=\left[2 \mathrm{P}^{2} / 3 \cdot \mathrm{GF}^{2}\right]$ and for $\mathrm{m}=4$ and $\mathrm{G}=2 \mathrm{E} / 5 \rightarrow \mathrm{W}=(7 / 4) \cdot[\mathrm{P}$ $\left.{ }^{2} / \mathrm{EF}^{2}\right], \mathrm{Wv}=(5 / 3) \cdot\left[\mathrm{P}^{2} / \mathrm{EF}^{2}\right]$.....(d)

Gravity`s Stationary Displacement Current $[\mathrm{E} \perp \mathrm{P}]$.

In [40-41] is shown the origin of this current and its relation to Gravity -Field and Gravity force . Since principal stresses $\sigma 1, \sigma 2$ are equal to the corresponding transverse velocities v 1,v2 which are the Cross product of a vector $\bar{v}$, so, $\bar{v} 1=(\bar{v} 1 / 2) \cdot[1+\sqrt{ } 5] \rightarrow \bar{v} 2=(\bar{v} 2 / 2) \cdot[1-\sqrt{ } 5]$ being the Odd and Even functions of points. The Norm is $|\mathbf{v}| \cdot|\mathbf{v}|=\mathbf{v}^{2}=(\mathrm{v} 1 / 2) \cdot[1+\sqrt{5}] .(\mathrm{v} 2 / 2) \cdot[1-\sqrt{ } 5]=(\mathrm{v} 1 . \mathrm{v} 2 / 4) .(1-5)=-\mathrm{v} 1 . \mathrm{v} 2$ or , $\overline{\mathrm{v}} \mathbf{2}=-\overline{\mathrm{v}} \mathbf{1} \cdot \overline{\mathrm{v}} \mathbf{2}$ and the analogous in Mechanics , $\sigma^{2}=-\sigma 1 . \sigma 2$..(e) i.e. Extreme equation (e) , joints at edge points the Principal stresses, $\bar{\sigma}$, and velocities, $\bar{v}$, into the quaternion type $\bar{z}=\bar{\sigma}=\bar{v}$ which is $\bar{z}=[\lambda, \pm \Lambda \nabla \mathbf{i}]$, in real $(\lambda)$ and imaginary part $[ \pm \Lambda \nabla \mathbf{i}]$ either as massive particle $[\lambda \mathrm{m}]$, or as Wave $[$ Energy $\pm \Lambda \nabla \mathbf{i}=\lambda]$ or both , and the conjugate quaternion $\bar{z}^{\prime}=\left[\lambda^{2}-\mid \Lambda^{2}\right]$ with equation e,o becoming, e,o $=[|\Lambda| \nabla,-\nabla \mathbf{x} \bar{\Lambda}]$ and defines the deep relation between, Energy as velocity $(\bar{v})$ and stress $(\sigma)$ and into both, Space as velocity $|\overline{\mathbf{v}}| \perp|\overline{\mathbf{v}}|$ or stresses $|\sigma 1| \perp|\sigma 2|$, and into the transverse equilibrium Anti-space as velocity $-|\overline{\mathbf{v}}| \perp-|\overline{\mathbf{v}}|$ or stresses $-|\sigma 1|$ $\perp-|\sigma 2|$.

This property of equation (e) in inner monad's wavelength $(\boldsymbol{\lambda})$ where $\mathrm{dF}=0$ results to the equation $\mathbf{c}^{2}=$ $\boldsymbol{\sigma}^{2}=\boldsymbol{\sigma} \mathbf{1} . \boldsymbol{\sigma} \mathbf{2}=\mathbf{v 1 . v 2}$ and it is a relation between constancy of velocity and the main stresses. Furthermore $\boldsymbol{\sigma} \mathbf{1}$ is a measure of how much in medium, the wavelength $\boldsymbol{\lambda}$ of monads where with $(+),(-)$ dipole moment creates the inner Electric and Magnetic Fields E ,P, opposes to any external E , B field and $\boldsymbol{\sigma} \boldsymbol{2}$ is a measure of the inner vortex density (w), the Spin of particle, of medium which opposes also to any external E , B field.

The inner Electromagnetic field of monads is produced from the two transverse moving velocity vectors $\overline{\mathrm{v}} 1, \overline{\mathrm{v}} 2$ on the two cycloids and consist Space-Quanta. From electromagnetic theory, Permittivity $(\varepsilon)$, is a Dielectric constant multiplier which affects the propagation of Electric fields, and it is a measure of how much the molecules oppose the external E-field due to a single point charge $\mathrm{q}[\mathrm{C}]$ at a distance $\mathrm{R}$ and exists, Electric flux density $\mathrm{D}=$ multiplier $(\varepsilon)$.Electric field $\mathrm{E}$.

Permeability $(\mu)$, is a measure (a scalar field) of the inner vortex density $\mathrm{w}[\square \mathrm{x} \overline{\mathrm{v}}]$ oppose the external $\mathrm{B}$-field due to a moving electric charge ,q, and exists, $\mathrm{H}=$ The pure vorticity (Spin), $\mathrm{B}=$ The weighted vorticity that is weighted for the density of the vortex $[\mathrm{MFMF}]$ field and conserved issuing $\mathrm{B}=\mu \mathrm{H}$.

By definition $\varepsilon=1 / \mathrm{q}=1 / \sigma 1$ and $\mu=1 / \mathrm{w}=1 / \sigma 2$ where then $\mathrm{c}^{2}=\mathrm{v}^{2}=\sigma^{2}=\sigma 1 . \sigma 2=\mathrm{v} 1 . \mathrm{v} 2=(1 / \varepsilon) .(1 / \mu)=1 \varepsilon$. $\mu$ or , $\mathbf{c}^{2} \cdot \boldsymbol{\varepsilon} \cdot \boldsymbol{\mu}=\mathbf{1}$ which is the known relation between the speed of light and Permittivity, Permeability, in free space medium ... [5]

\subsection{The Conserved Magnitudes .}

Let, $R(a)=$ The Place (Position) of the System (a), i.e. the Distance, from a reference point 0 . U (a) = The total Impetus of System (a). La = The Impulse of any System from a fixed beginning, reference , point 0 .Via two Systems (a) and (b) , or any dipole systems (a),(b) is holding, Total Impulse $\mathrm{L}=\mathrm{La}+\mathrm{Lb}=\mathrm{R}$ (a).P (a)+R (b).P(b) = R (a).[m (a). $\gamma(a)]+R(b) \cdot[m(b) \cdot \gamma(b)]==R(a) \cdot F(a b)+R(b) \cdot F(b a)=0$ or $\rightarrow R(a) \cdot F(a b)-R$ (b). $F \cdot(a b)=R(a b) . F(a b)=0$ because Equilibrium, and since $F(a b)=m(a) \cdot \gamma(a), F(b a)=m(b) \cdot \gamma(b)$ hence is $R(a) \cdot[m(a) \cdot \gamma(a)]+R(b) \cdot[m(b) \cdot \gamma(b)]=0$ and is in effect $\rightarrow R(a) \cdot U(a)+R(b) \cdot U(b)=$ Constant i.e.

The Work $\rightarrow$ as Impetus , Angular-Momentum and Energy $\leftarrow$ produced in all Systems , is Conserved , ( Quantized ), But How ??

Since A-M = Angular-momentum = is Work and is conserved , therefore Material Points, which such are the Particles, are unaffected when rotating .

This Property of axis of rotation becoming from the Spinning-Wheel-Rim, to be free to assume any Orientation exists only on Gyroscope-Mechanism, where The three RIM-Frames, with one and two degrees of rotational freedom, are maintaining, tilting rotation of mounting according to A-M axis. And because of the ,Succession Shifting of Positions, then Monads are Energy-Quanta in the Double-helix Space-Quanta Gyroscopic-Mechanisms . Material Geometry the Periodic Table and Chemistry .

\section{A Comparison Chart between Euclidean Geometry and Material Geometry : E-Geometry Material Geometry}

A Point $\rightarrow$ Is nothing Are energy Breakages, cells $\bigoplus=+(w r)^{2}, \Theta=-(w r)^{2} \&=\bigoplus \Theta=2(w r)^{2} \neq \pm$ i.e. neutral to them, Zero i.e. Zero is the collision of the opposite breakages Position of Point $\rightarrow$ Every where Within the objective reality is the quantized energy A Line $\rightarrow$ Is of Infinite, $\infty$,points Is of Discrete points $\oplus \oplus \oplus, \ominus \ominus \ominus, \oplus \ominus \oplus \ominus$ Direction $\rightarrow$ Are all directions x,y,z forward and backward . Is in one direction in each axis as,$\oplus \rightarrow \leftarrow \Theta$. A Plane $\rightarrow$ Is of three non-coinciding Points It is of three Points with the Zero 
fragments $\oplus, \ominus$ Steady $\rightarrow$ Is the triangle becoming from the extrema points of line to equilateral triangles Zero of opposite ,on the three vertices, i.e. hexagon Frequency in change of direction $\rightarrow$ Exist infinite reversed directions in any plane .

Exists the Zero direction in Hexagon steady -plan and a second flowing change that in material points Obtained from $\rightarrow$ Is of Point which is nothing and of Imaginary nature .

Material points of Quaternion nature as shells or tiny caves ,or Work included in them Amount of Extension $\rightarrow$ Is from zero to \pm Infinite or symbolism $0 \rightarrow \pm \infty=-\infty \leftarrow 0 \rightarrow+\infty$ It depends on the Potential difference , the Voltage, contained in material cells or the number of Shells.

Case of Motion $\rightarrow$ It is that Diversity or varying of Points position in all directions or forward It is the alternative uniqueness of mutual traction creating Work on constant magnitude $\oplus$ to $\Theta$, the cells $=$ breakages , in one direction or and forward The result is $\rightarrow \mathbf{A}$ sector $\mathbf{d s}$ can be repositioned Infinite times on a straight or a curved line.

The motion of monad, the material sector, through these discontinuously created from inner, $\oplus \leftrightarrow \ominus$, monad, and Externally acting on monad. The Correlation between , Euclidean and Material-Geometry on Points , Lines , Planes, Volumes -

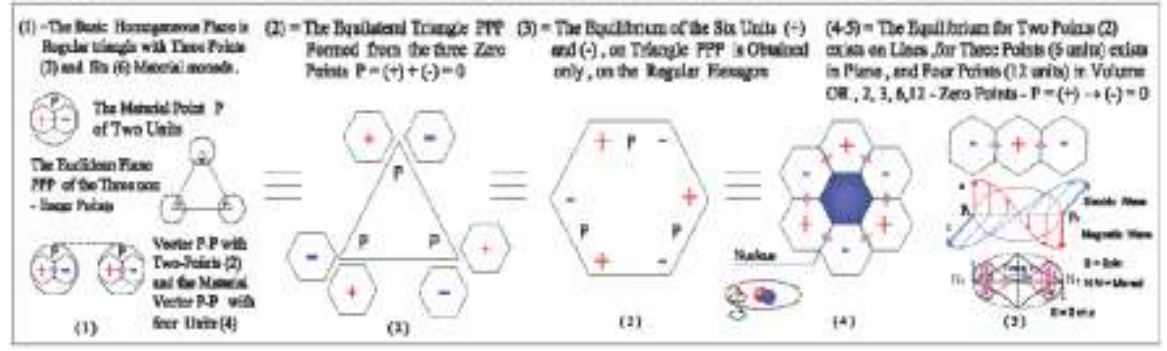

Figure.1

The Stationary Material Point $[\bigoplus \ominus]=0$, The two Units $(\mathrm{U})$ on $\rightarrow$ Euclidean Points (2U) $\equiv$ Zero Line Sector (4U), Plane (6U), Volume (8U), N Spaces (2N.U) $\equiv$ Point, Sector, Plane, Volume, Sphere. N -Space in EGeometry is defined any shape formed by the Points not coinciding . The Permissive Sites within the premises are the Available Positions in each Level $\equiv$ Mould .

[1]. The One Point is Zero=0, while Material-Point is the sequence of Breakage`s length $\bigoplus \ominus$ The Two points, Sector, is the length ds $=\mathrm{PP}$, while Vector $\mathrm{P \overline {P }}=$ Material length $=$ Monad $=$ Quaternion $=$ The sequence Breakage`s length $\mathrm{r}=\mathrm{PP}=\bigoplus \leftrightarrow \Theta---\bigoplus \leftrightarrow \Theta$ of 4 units .

[2]. Three Units = One point and a unit , is the cause of inner motion and Spin of monads. The Three Points not coinciding, form the Plane of three sectors by two, equal 6 units The Four Points in Plane, form a Shape of four sectors by two, equal to 8 units .

[3]. The Three points, sequence the stable shape of equilateral triangle with 6 units $\bigoplus$ and $\ominus$.

[4]. The three points, sequence the stable shape of equilateral triangle with the Zero Breakage`s

[5]. length $\{\oplus \leftrightarrow \Theta=$ Zero $\}$ on the three vertices of the triangle. For monads ds $=r=\leftrightarrow$

[6]. The three Points not coinciding, form the Plane of three sectors by two, equal to 6 units

[7]. The Four Points not coinciding, form Volume of six sectors by two, equal to 12 units .

[8]. Equilibrium exists by the three reciprocating Sources units $\bigoplus$ to the three drain $\ominus$ units.

[9]. The three Units of One point and a unit , or a Positive Breakage between two Negative units [ $\Theta \leftrightarrow \oplus \leftrightarrow \Theta]$ causes the Inner Electromagnetic Wave, which creates on axis Spin as a Torque causing motion of monads.

\section{Remarks :}

All material points are produced from \pm Breakages which consist the $\oplus, \Theta$, Units as follows , Breakage $\mathrm{s}^{2}=+(\mathrm{wr})^{2}=$ The Positive $\oplus$ Unit Breakage $-\mathrm{s}^{2}=-(\mathrm{wr})^{2}=$ The Negative $\Theta$ Unit $\oplus \leftrightarrow \Theta={ }^{\circledR}=$ The Rest Energy Quanta Breakage $2 s^{2}=2(w r)^{2}=$ The Energy Unit Mutual traction of Breakages [+(wr) $\left.{ }^{2}\right] \leftrightarrow[-$ $\left.(\mathrm{wr})^{2}\right]=\oplus+\Theta=\oplus \Theta=\& \rightarrow$ The Neutral Unit Unit $\rightarrow \oplus \equiv \&-\Theta=[\oplus \Theta]+[-\Theta] \rightarrow$ One Neutral Energy Quanta and one Anti-negative Unit Unit $\rightarrow \ominus \equiv \&-\oplus=[\oplus \Theta]+[-\oplus] \rightarrow$ One Neutral Energy Quanta and one Anti-positive Unit

Collision of Breakages $\left[+(w r)^{2}\right] \rightarrow \leftarrow\left[-(w r)^{2}\right]=\bigoplus{ }^{\prime} \Theta=\bigoplus \Theta=\&$ The Neutral Energy Quanta

Collision of Breakages [-(wr) $\left.{ }^{2}\right] \rightarrow \leftarrow\left[-(w r)^{2}\right]=\Theta{ }^{\prime} \Theta=\Theta+\oplus+\phi \rightarrow$ Two $\Theta$ Units +Energy

Collision of Breakages $\left[+(\mathrm{wr})^{2}\right] \rightarrow \leftarrow\left[+(\mathrm{wr})^{2}\right]=\bigoplus$

$\rightarrow \oplus+[\oplus \Theta] \rightarrow \oplus+\&=$ One Positive and One Neutral Unit,$\rightarrow+[-\ominus] \rightarrow$ One Positive Negative-Unit $=$ The Positive Anti-negative Space Unit,$\rightarrow$ The Energy Unit 


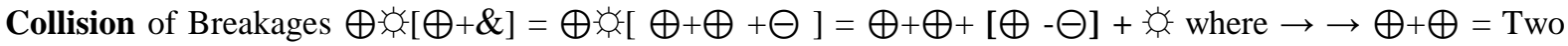
Positive Units, $\rightarrow[\oplus-\Theta]=$ The one Neutral Unit,$\rightarrow=$ The Energy Unit ,

Collision of Breakages $[\oplus+\oplus+\&]$

$\rightarrow \oplus+\oplus+\square+\square=$ Two Positive Units and Two Neutral Units ,

$\rightarrow[\oplus+\oplus]=$ Two Positive Units ,

Addition of two opposite numbers in Geometry $\equiv$ Number $(+)+(-)=0$ causes Neutral Unit, i.e. Zero Addition of two Rest Breakages $\equiv$ Units $\oplus+\Theta=0=\oplus \leftrightarrow \Theta=\square$, and causes Neutral or Zero Unit.

Addition of three Rest Breakages in Geometry $\equiv$ Number $(-)+(+)+(-)$ causes any Number of the Linear Coordinate System , Positive , Negative or Zero i.e. the Straight line .

Addition of three Rest Breakages $\equiv$ Units $\Theta \leftrightarrow \oplus \leftrightarrow \ominus \equiv \Theta \leftrightarrow \&=\& \leftrightarrow \oplus=\mathrm{V}^{\leftrightarrow}$, causes Vibration and Spin

1. In all above cases has been used the , E-Geometry Logic , for Summation and its properties.

Using the logic of E-geometry Elements then is found $\rightarrow$ what is Energy -Space, The How, When and Why Energy is Quantized in Space, The Origin of Material Geometry which is the objective reality of Cosmos, and

the why Geometry Mechanics and Physics faithfully follow.

Material Geometry the Periodic System and Chemistry . 11

Photo is from -Resolution Of The Russian Theory Of Microworld -By Professor Ph. M. Kanarev

[ Kanarevfm@Mail.Ru]
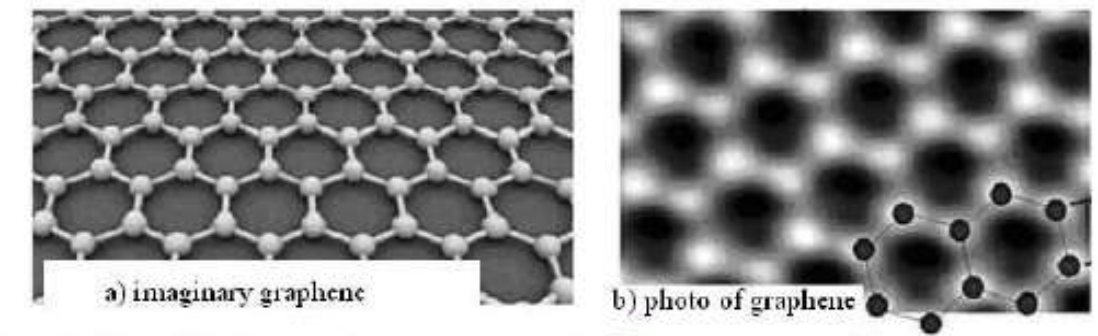

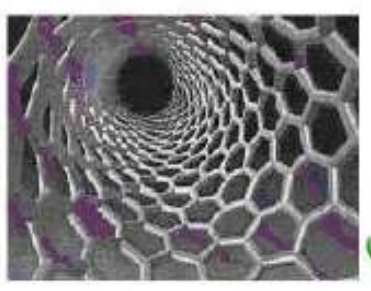

d) graphene tube

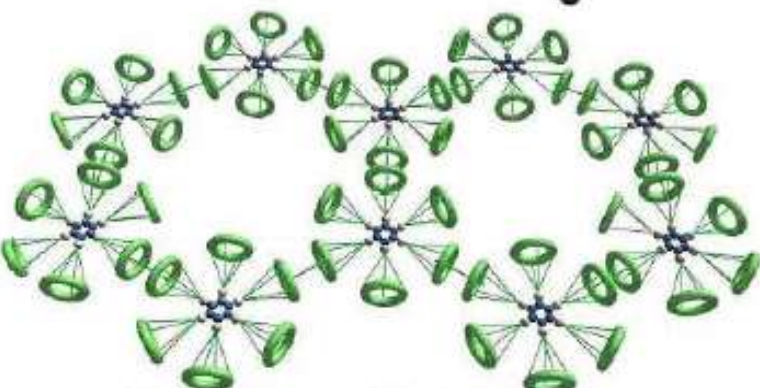

c) theoretical details of the photo of graphene

Figure.2

a)Is shown the structure of graphene (the Regular Hexagon of E-geometry) in a higher Level.

B Is shown the Regular Hexagon structure with Nucleus ,Core, to be the Torsional missing link of Cluster.

c) Is shown the Hexagon structure becoming from the triangle,times, two Units each point and equal to Six units, or (3 vertex x 2 units $=6$ Units), for any compound Geometrical Cluster .

e)The Hexagon shape is because in Photos is included the Projective Plane of graphene only.

\section{Remarks :}

1The Linear Level, Shell , configuration is of $\mathbf{8}$ available Units.

2The Plane Level, Shell, configuration is of 18 available Units.

3The Volume Level, Shell, configuration is of $\mathbf{3 2}$ available Units

4 The Hexagon shape comes from the Projection of Units in the Available-Positions of the flattened, Plane Level configuration. Flat Plane corresponds to an Angular-Node that goes through the Orbital .

[ According to Bohr`s model $\rightarrow$ Electrons orbit the nucleus in Shells ]

5 The elevated positions at nodes due to the ,Volume-Level, is generated by the two The Material Geometry and the Origin of Energy-Particles in Periodic System 12 


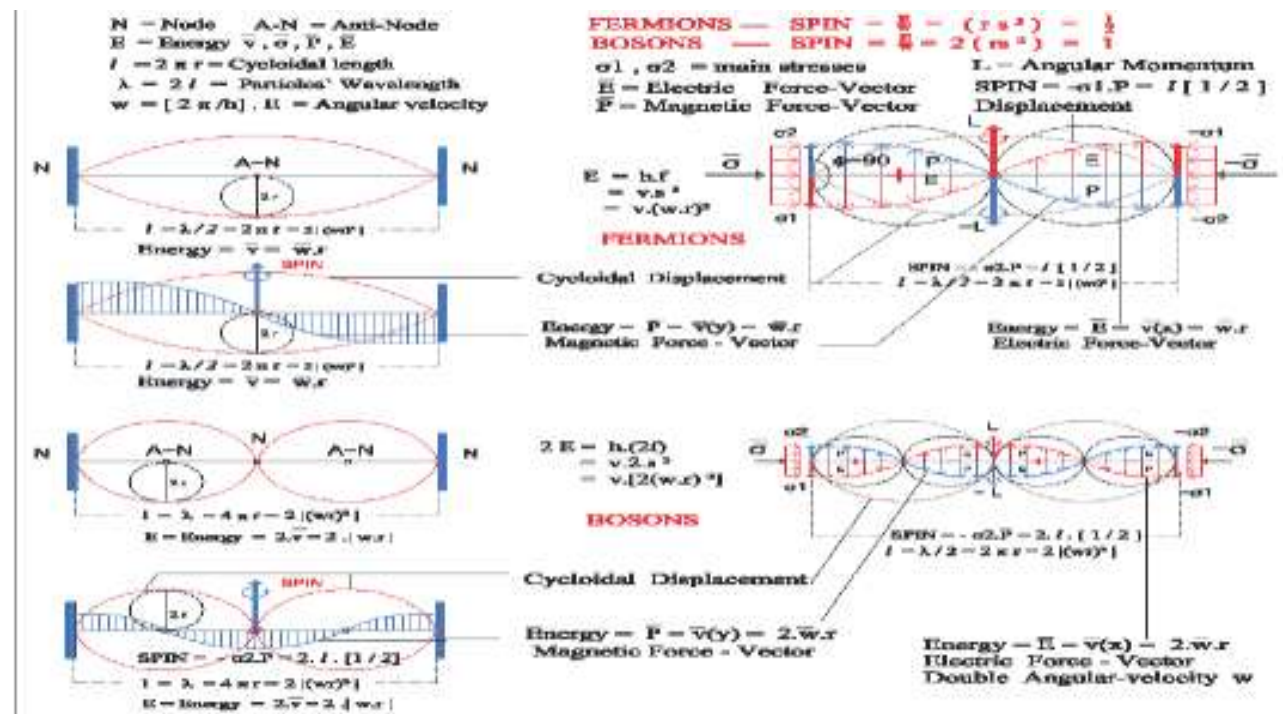

Figure.3 : Energy, as Stress $=$ velocity v $=(w r)$ creates Spin $1 / 2$, while Doubled, v $=2(w r)$ creates Spin 1 .

\section{Stationary Waves}

Let us consider the length of a cave, 1 , and see the relationship to the wavelength,$\lambda$, and frequency ,f. In a cave of length , 1 , where vibrations are of two fixed nodes, one pair at each end, and since also $c=f . \lambda$ then exist for ,

First Harmonic $\mathrm{f} 1 \rightarrow 1=N / 2=\mathrm{c} /(2 . \mathrm{f})$

$\mathbf{f} \mathbf{1}=\mathrm{c} /(2 \mathrm{l})=\mathbf{f} \mathbf{1}$ and

Second Harmonic f $2 \rightarrow 1=\lambda=\mathrm{c} /$ ( f ) and

f $2=\mathrm{c} /(1)=\mathbf{2 . f} \mathbf{1}$

Third Harmonic $\mathrm{f} 3 \rightarrow \mathrm{l}=3 \mathrm{~N} / 2=3 \mathrm{c} /(2 \mathrm{f})$ and

f $3=\mathrm{c} /(1)=$ 3.f 1 Material Geometry the Periodic System and Chemistry .13

Fourth Harmonic f $4 \rightarrow 1=4 N / 2=4 \mathrm{c} /(2 \mathrm{f})$ and $\mathbf{f} \mathbf{4}=\mathrm{c} /(1)=\mathbf{4 . f} \mathbf{1}$

Fifth Harmonic $\mathrm{f} 5 \rightarrow 1=5 \mathrm{~N} / 2=5 \mathrm{c} /(2 \mathrm{f})$ and

f $5=\mathrm{c} /(1)=\mathbf{5 . f} \mathbf{1}$

The n.th Harmonic $\mathrm{f} n \rightarrow 1=\mathrm{n} N / 2=\mathrm{nc} /(2 \mathrm{f})$ andf $\mathbf{n}=\mathrm{c} /(1)=\mathbf{n . f} \mathbf{1} \mathrm{f} \infty \rightarrow 1=\infty N / 2=\infty . \mathrm{c} /(2 \mathrm{f})$ and $\mathbf{f} \infty=\mathrm{c} /(1)=\infty$.f 1

All of the particles between two nodes vibrate in phase with each other, but the amplitude of vibration varies from zero at the node to maximumat the antinode, back down to zero at the second node, while neighboring, lobes, are in antiphase each other .

The number of notes that will be present along the medium, the cave, is depended upon the number of crests that are present in between the two timed crests.

\subsection{The Standing Waves .}

Equation of Gravity, which is the minimum and less attractive Force in a Standing wave, equal to $i=$ $2(\mathrm{wr})^{2}$ on dispersion $\rightarrow\left[\left|+(\overline{\mathbf{w} . r})^{2}\right| \leftrightarrow\left|-(\overline{\mathbf{w . r}})^{2}\right|=|\lambda|\right]$ cycloidal cave $\leftarrow$ and which cave is the material energy length consisted of the material end points $\left|(\mathbf{w r})^{2}\right|$ and are the two $\pm\left|(\mathrm{wr})^{2}\right|$ dipole , restrained by the above Electromagnetic field E,P , and becoming from the binding force , $\left|2(\mathrm{wr})^{2}\right|$ are as follows ,

$\mathrm{Y}=[2 \mathrm{~A} \cdot \sin (\mathrm{kx})] \cdot \cos (\mathrm{wt})$ where $\mathrm{A}=\mathrm{v} 1=\mathrm{v} 2=|\mathrm{v}| / \sqrt{ } 2 \rightarrow$ Wave`s Amplitude, $\mathrm{kx}=\pi / 2, \pi, 3 \pi / 2, \rightarrow$ The Wave`s number, location, $w=2 \pi f=2 \pi / T \rightarrow$ Oscillating frequency, angular frequency, and equations for E,P field are

$\mathrm{E}=2 \mathrm{~A} \cdot \sin k \mathrm{x} \cdot \cos \mathrm{wt}=\sqrt{2} \cdot\left|(\mathrm{wr})^{2}\right| \cdot \sin k x \cdot \cos w t$,

$P=2 A \cdot \sin k x \cdot \cos w t=\sqrt{ } 2 \cdot\left|(w r)^{2}\right| \cdot \sin k x \cdot \cos w t$,

$\sin (\mathbf{k x}) \rightarrow$ is the Spatial dependence at locationsx $=0, N / 2, \lambda, 3 N / 2$ called the nodes. At nodes amplitude , A, is always zero and at locations $\mathrm{x}=N 4,3 N / 4,5 N / 4$ called the Anti-nodes where the amplitude becomes maximum .

$\cos (\mathbf{w t}) \rightarrow$ is the Time Oscillation dependence .

Since energy $E=h . f$ then frequency $f=E / h$. Angular velocity $w=2 \pi T=2 \pi . f=2 \pi .(E / h)=$ $\mathrm{E} /[\mathrm{h} / 2 \mathrm{~T}]$, or 
$\mathbf{w}=\mathbf{E} /(\mathbf{h} / \mathbf{2} \pi), \mathbf{E}=\mathbf{w} \cdot[\mathbf{h} / \mathbf{2} \pi]$..(a) i.e

Proportional to energy $\mathrm{E}$, since $\mathrm{h} / 2 \mathrm{~m}$ is constant.

This proportionality of (w, E) is the Why the crests of a Quantized magnitude of energy, define the number of notes that will be present along the medium, it is the cave of length $1=4 \pi \mathrm{r}$, and of volume $323\left(\pi^{2} \mathrm{r}^{3}\right)$ dependent only on caves-radius r. Standing Waves, can occur when the medium is moving in the opposite direction to the wave, or it can arise in a Stationary medium, caves, as a result of interference between two waves travelling in opposite directions .

A Standing Wave, or a Stationary Wave -Is a wave that has parts that remain in a constant position .

Nodes -Points of no displacement -remain in the same position where Particles of these points are not vibrating at all. This definition explains the why Electric and Magnetic fields are also perpendicular.

Anti-Nodes -Points of maximum displacement--between each pair of nodes on the waveform where the Particles suffer maximum to $\mathrm{x}$ - $\mathrm{x}$ perpendicular displacement and are in vibration .

Since Energy in a cave, stands still and also is conserved, then from Planck`s equation $E=$ h.f , where $-E, f-$ are constants and, the frequency ,f, is analogous to energy, $\mathrm{E}$ or $\mathrm{f}=\mathrm{E} / \mathrm{h}$.

\subsection{Intrinsic Angular Momentum and Spin .}

Intrinsic Spin is the Quantum version of Angular Momentum, $\mathrm{L}=[\mathrm{h} / 2]=\mathrm{r} \cdot \mathrm{p}=\mathrm{r} .(\mathrm{m} . \mathrm{v})=(\mathrm{r} \cdot \mathrm{m}) \cdot \mathrm{v} . \mathrm{A}$ ,quanta, is the smallest unit of something and, as it happens, there is a smallest unit of angular momentum equal to $\mathrm{h} / 2$. Equation (a), $\mathrm{w}=\mathrm{E} /[\mathrm{h} / 2 \mathrm{~T}]$ defines the number of notes that will be present along the medium , the cave, and is depended upon the Incident energy , $\mathrm{E}$, on cave so , $\mathbf{w}=\mathrm{E} /[\mathrm{h} / 2 \pi]=\mathbf{E} \cdot[2 \pi / \mathrm{h}]=\mathbf{E} / \mathbf{S P I N}$

When the applied force $\mathbf{E}=h f=\mathbf{w} .(h / 2 \pi)=w . S p i n$ then $\mathbf{S p i n}=\mathbf{E w}=\left[ \pm \bar{v} . s^{2}\right] / \mathrm{w}=\left(\mathrm{r} . \mathrm{s}^{2}\right)$ on cave and are created Fermions with Spin $=[\mathrm{Ew}]=\left(\mathbf{r} . \mathrm{s}^{2}\right) \rightarrow 12$

When the applied force $\mathbf{E}=$ h.f $=\mathbf{w} \cdot(\mathrm{h} / 2 \pi)=\left[\nabla i=2(\mathrm{wr})^{2}=2 \cdot \bar{v} s^{2}\right]$ on cave then $\mathbf{S p i n}=\mathbf{E} \mathbf{w}=\left[2 \cdot \bar{v} \cdot s^{2}\right]$ $/ \mathrm{w}=2 .\left(\mathrm{r} . \mathrm{s}^{2}\right)$ and are created Bosons with $\operatorname{Spin}=[\mathbf{E w}]=\mathbf{2} .\left(\mathbf{r} \cdot \mathrm{s}^{2}\right) \rightarrow \mathbf{2 .}(\mathbf{1} / \mathbf{2})=1$ which is double the before

i.e. Energy as velocity vector $\bar{v}$ or $\mathbf{E}=[ \pm \bar{v}]$, applied on the material energy dipole points $\left[ \pm s^{2}\right]$ as the Dipole Quantity $|\overline{\mathrm{v}}|^{2}=|\overline{\mathrm{w}} \times \overline{\mathrm{r}}|^{2}=|\overline{\mathrm{w}} \cdot \mathrm{r}|^{2}=\mathrm{s}^{2}$, separately creates the massive Particles, Fermions, while the velocity vector $\overline{\mathrm{v}}$, applied on the Double Quantity The Material Geometry and the Origin of Energy-Particles in Periodic System 14 Energy vector breakages $\nabla i=2(w r)^{2}=2 \cdot s^{2}=2[ \pm \bar{v}]^{2}=\mathbf{2 E}$ and since $v=(w \cdot r), 2 v \cdot s^{2}=2|\bar{v}| s^{2}=2 \cdot|\bar{w} \times \bar{r}|^{3}$ $=2 \mathrm{v} \cdot|\overline{\mathrm{w}} \cdot \mathrm{r}|^{2}=2 \mathrm{~s}^{2}$ creates the Energy Particles, the Bosons, by doubling its frequency [ f $\left.2=2 . \mathrm{f} 1\right]$ in the same cave.

The both rest, Gravity Field and Dark-matter are consisted of the same material points jointed by the inward E,P fields, which two , consist Gravity`s and Dark-matter`s rest material dipole. Figure.8 From Planck`s energy equation $E=h . f=(h / \lambda) \cdot c$ and Isochromatic pattern $\sigma 1-\sigma 2=(a / d) \cdot N=(a / d) \cdot n . f 1$, where the Isochromatic fringe Quantized order n.f1=(wr $)^{2}$, is varying from the First-Harmonic Energy-Bunched variation $\mathrm{f} 1 \rightarrow \lambda / 2$ to Second $\mathrm{f} 2 \rightarrow \lambda$ for doubled Energy and to n.f1. [40] The Standing waves in caves $1=\lambda 2 \rightarrow \lambda$, and the Intrinsic Angular momentum of Particles In Figure. 3.

[1] The applied force on NN cave is $\mathrm{E}=\mathrm{h} . \mathrm{f}=\mathrm{w} .(\mathrm{h} / 2 \mathrm{~T})=\mathrm{w} \cdot \mathrm{SPIN} \rightarrow \mathrm{Spin}=\boldsymbol{E} \boldsymbol{w}=\left[ \pm \overline{\mathcal{v}} \cdot \mathrm{s}^{2}\right] / \mathrm{w}=\left(\mathrm{r} \cdot \mathrm{s}^{2}\right)$

[2] For $\mathrm{E}= \pm \bar{v}$ then $\rightarrow$ Spin $=\boldsymbol{E} \boldsymbol{w}=\left[ \pm \bar{v} \cdot \mathrm{s}^{2}\right] / \mathrm{w}=\left( \pm \mathrm{r} . \mathrm{s}^{2}\right) \rightarrow \pm$ Fermions with spin 12

[3] For $\mathrm{E}=\left[\nabla \mathrm{i}=2(\mathrm{wr})^{2}=2 \cdot \overline{\mathrm{vs}^{2}}\right]=2 .\left(\mathrm{r} \cdot \mathrm{s}^{2}\right)$ then $\rightarrow \mathrm{Spin}=\boldsymbol{E} \boldsymbol{w}=\left[2 \cdot \bar{v} \cdot \mathrm{s}^{2}\right] / \mathrm{w}=2 .\left(\mathrm{r} \cdot \mathrm{s}^{2}\right) \rightarrow$ Bosons of spin 1

i.e. Double energy [2.(r.s $\left.\left.\mathrm{s}^{2}\right)\right]$ on a constant cave creates 2 crests and doubling the frequency (h), with Spin 1 . Ntimes energy $\left[\mathrm{N} .\left(\mathrm{r} . \mathrm{s}^{2}\right)\right]$ on a constant cave creates $\mathrm{N}$ crests $\mathrm{N}$-times the frequency (h) with Spin N/2. Since Energy $[\overline{\boldsymbol{E}} \mathbf{X} \overline{\boldsymbol{H}}]=$ Pressure $=$ Spin $\mathrm{S}=$ p.c.w. $\left[\varepsilon \mathrm{E}^{2}+\mu \mathrm{H}^{2}\right] / 2=2$ rc. $\sin .2 \varphi \rightarrow$ then Energy $/ \sin 2 \varphi=\left[\varepsilon \mathrm{E}^{2}+\mu \mathrm{H}^{2}\right] /$ $\sin 2 \varphi=2 \mathrm{rc} / \rho \mathrm{w}=4 \mathrm{r}^{2} / \rho=$ constant , happening only on Cycloidal motion .

It was shown that equation of a space wave is $\rightarrow A x, t=y=A o \cdot \sin (k x-w t+\varphi)=$

$\operatorname{Ao} \cdot \sin [2 \pi(x \lambda-t T)+\varphi)]=\operatorname{Ao} \cdot \sin [(2 \pi \lambda) \cdot(x-c t)+\varphi]$

where $\varphi$ is the phase and $\mathrm{T}$ the period of the wave

Wave's velocity $\bar{v}=\dot{y}=(-w A o) \cos [(2 \pi \lambda) \cdot x-w t]=(-w A o) \cos [(2 \pi \lambda) \cdot(x-c t)]$ and Energy of wave is , E $=m \cdot \dot{y}^{2} / 2=(\mathrm{m} / 2) \cdot(-\mathrm{wAo})^{2}$.

By definition $\rightarrow$ Pressure $(p)=$ Intensity of a force $(/ p e r)$ area, Density $(\rho)=$ Intensity of a mass(energy)/volume and Intensity of waves is Intensity (I) = Power delivered / unit area so, Intensity of a Wave is,$=$ werArea $=$ Energy.LengthTime. Volume $=($ Energy $)$.Length $($ Volume $)$.Time $=$ EnergyVolume $($ speed $)=$ $(\mathrm{m} / 2) .(-\mathrm{wAo})^{2} \cdot \overline{\mathrm{v}} /$ volume $=($ p.c2 $) .(\mathrm{wAo})^{2}=[2 \pi 2 . \rho . c 3] . \mathrm{A}^{2} \lambda^{2} \rightarrow$ since $\mathrm{w}=(2 \pi / \mathrm{T})=2 \pi \mathrm{c} / \lambda$. , and in

case of the tiny volume $\mathrm{V}=\mathbf{4} \lambda^{3} 3 \pi$, then $\mathrm{A}^{2}=\left[\varepsilon \cdot \mathrm{E}^{2} 2+\mu \cdot \mathrm{H}^{2} 2\right]^{2}=\left[\varepsilon \mathrm{E}^{2}+\mu \mathrm{H}^{2}\right] / 4$, and mass/volume $=\rho=\varepsilon, \mu$,of inner cycloidal structure and intensity $I=(\mathrm{m} / 2)(-\mathrm{wAo})^{2} \mathrm{v} /$ volume $=\rho \mathrm{r}^{2} \mathrm{c}^{3} 2 \lambda^{2}\left[\varepsilon \mathrm{E}^{2}+\mu \mathrm{H}^{2}\right]$

$=\rho^{2} T^{2} c^{3} 2 \lambda^{2}$, and for $\rho=\left[\varepsilon E^{2}+\mu H^{2}\right]$, and also $\lambda=(w r)^{2}$, The pressure Amplitude Pd= p.c.w.Ao= p.c.w. $\left[\varepsilon E^{2}+\mu H^{2}\right] / 2=\rho^{2} . c . w / 2$ 


\section{Intensity of Dark-Fringes Id $=\rho^{2} \pi^{2} c^{3} 2 \lambda^{2}$}

The Impedance (Resistance is attributed to resting bodies and Impetus to moving bodies) of the medium is defined by the product of density and wave speed as $\mathrm{z}=\rho$.c with a unit of $\rightarrow$ Pa.s.m -1 and Pressure amplitude $\mathrm{Po}=$ p.c.w.Ao .

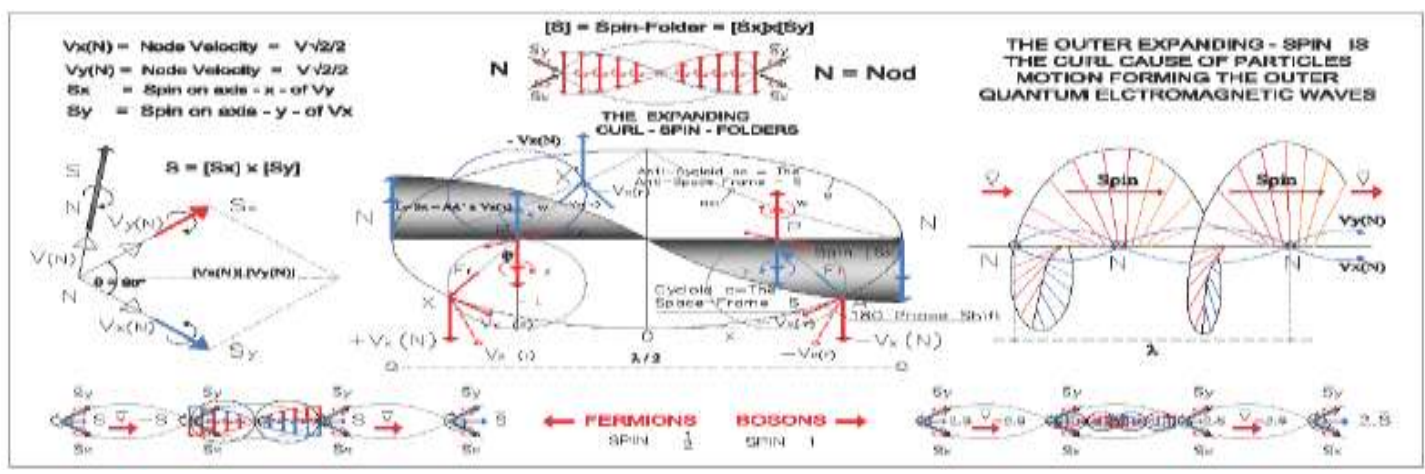

Figure. 4.

The Mechanism of Angular Momentum - Spin - in Particles and their Curl-motion .

A : Velocity $\mathrm{V}$ as thrust is acting on primary elements $\mathrm{s}^{2}=(\mathrm{wr})^{2}=N / 2$, decomposed to $, \mathrm{Vx}, \mathrm{Vy}$, by $\mathrm{C}-\mathrm{P}$.

B : The couple of Tangential velocities $\mathrm{Vx}(\mathrm{T}),-\mathrm{Vx}(\mathrm{T})$ on Cycloid, Evolute, creates spin $\mathrm{Sx}=\mathrm{Vx}(\mathrm{T})$. XX'.

$\mathbf{C}: \overline{\mathrm{S}} \mathrm{x}, \overline{\mathrm{S} y}$ spins are composed to Spin $\overline{\mathbf{S}}$, as the Outer-Folders-Spin, following $\mathrm{C}-\mathrm{P}=$ Cross Product .

D : $\bar{V} x(r), \bar{V} y(r)$ Centripetal vectors create the Inner Quantum Electromagnetic Waves of monads .

Spin $=\mathbf{E w}=\left[ \pm \overline{\mathrm{V}} \cdot \mathrm{s}^{2}\right] / \mathrm{w}=\left(\mathrm{r} . \mathrm{s}^{2}\right)$ and for gravity level $\mathrm{r}=10-62 \mathrm{~m}$, the relative strength is not detected.

The maximum angular momentum $\mathrm{Lmax}=\mathrm{Spin}=\mathrm{S}$ is created from the tangential force $\mathrm{F}(\mathrm{T})$ at Nods ,N, and their evolute , $N^{`}$, and since mass of monads $m p=V \cdot \rho=323\left(\pi \mathrm{r}^{2} \mathrm{r}^{3}\right) \cdot\left[\varepsilon \mathrm{E}^{2}+\mu \mathrm{H}^{2}\right]$ and spin $\mathrm{S}=(\mathrm{h} / 2 \pi)=\mathrm{the}$ reduced Planck constant, then, $\mathrm{S}=\mathrm{F}(\mathrm{T}) \cdot 2 \mathrm{r}=[\mathrm{mp} .(\mathrm{c} / \sqrt{2} / 2)] .4 \mathrm{r}=323\left(\pi^{2} \mathrm{r}^{3}\right) \cdot\left[\varepsilon \mathrm{E}^{2}+\mu \mathrm{H}^{2}\right] \cdot 2 \mathrm{r} .(\mathrm{c} / \sqrt{ } 2)$, and the SpinEnergy-Density $=\left[\boldsymbol{\varepsilon} \mathbf{E}^{2}+\boldsymbol{\mu} \mathbf{H}^{2}\right]$ becomes $\left[\varepsilon E^{2}+\mu H^{2}\right]=3 \cdot(\mathrm{c} / \sqrt{2}) \cdot(\mathrm{h} / 2 \pi) / 32 \pi^{2} \mathrm{r}^{3}=3 \cdot c \cdot S / 3 \sqrt{2} \pi^{2} \mathrm{r}^{3}=3 \cdot$ c.S $32 \cdot \sqrt{2} \cdot \pi_{2} \cdot \mathrm{r}^{3}$ $=3 \cdot 3 \cdot 10^{\wedge} 8 \cdot S 32 \cdot \sqrt{ } 2 \cdot \pi 2 \cdot \mathrm{r}^{3}=2 \cdot S \cdot 10^{\wedge} 6 \mathrm{r}^{3}=1,335 \cdot 10^{-} 27 \mathrm{r}^{3}=1,335 \cdot 10^{-} 2712,088 \cdot 10^{\wedge-} 144=1,104 \cdot 10116 \mathrm{~J} / \mathrm{m}^{3}$ ....(Sed) For the next cave $\left(2,295.10^{\wedge}-48\right)$ to Planck`s cave length $\left(8,906.10^{\wedge}-35\right)$ the Energy density becomes $\left[\varepsilon E^{2}+\mu \mathrm{H}^{2}\right] / \mathrm{c}^{2}=3 . S c .32 \sqrt{ } 2 . \pi 2 \mathrm{r}^{3}=1,23.1099 \mathrm{Kg} / \mathrm{m}^{3} . .(\mathrm{Ed})$ Following Newton`s third law for motion , <in any action there is an equal and opposite reaction > for Angular momentum, the Spin, which is the Intrinsic Quantum-Scalar Wave field or ,the Quantum-Vector Electromagnetic field of monads, and which is acting on monad NN $=N / 2=s^{2}=(w r)^{2}$, is forming Anti-spin as Reaction .This Reaction, the Anti-Spin $=$ force , is changed into the same monad as the Intrinsic Electromagnetic wave of monads NN.

In Figure. 4 , Work $\mathbf{W}$ as Energy, is the quantized velocity vector $\overline{\mathbf{v}}$, which is decomposed in $\mathbf{N}$, to the two transverse velocity vectors $\overline{\mathbf{v}}, \overline{\mathbf{v y}}$, which create the Inward Quantum wave-field $\mathbf{E} \square \mathbf{P}$, in tiny space $\mathrm{s}^{2}$ $=(\mathrm{wr})^{2}$ as resonance between $\mathbf{N N}$ nodes and termed at $\mathbf{N}$, where reflect and change $180^{\circ}$ phase, converging at initial node. For inner stability, the couple of the two tangential to Cycloid , Anti-cycloid velocities $\overline{\mathrm{V}} \mathrm{x}$ (T) , $\overline{\mathrm{V}} \mathrm{y}(\mathrm{T})$ and $-\overline{\mathrm{V}} \mathrm{x}(\mathrm{T}),-\overline{\mathrm{V}} \mathrm{y}(\mathrm{T})$ create Inner Spins Sx,Sy in velocities plane which are then composed to the Outer Spin of monad to become the Outward wave travelling at light speed and thus repeating the cycle again .This is the way of Work, as Force, as velocity vector and as Intrinsic-Spin of particle, to be converted to External and to move. The Material Geometry and the Origin of Energy-Particles in Periodic System 16 Remarks : Figure.4-5 a.. From definitions, Quantum-Spaces, or, Quantum-Monads are the Scalars $\left[ \pm s^{2}= \pm(w r)^{2}\right]$ and Vector [ $\square \mathrm{i}=2$. $(\mathrm{wr})^{2}$ ] breakages all quantized through [STPL] E-geometry [SS] Mould . Thrust is , Energy applied on breakages as velocity vector $\bar{v}$. Wave-Structures, are the clashed homogeneously

breakages, with the velocity vector $\bar{v}=\bar{c}$.

Spin is fixed and independent of particle`s mass because Spin $=(h / 2 \pi)$.w , or its external angular velocity, w, is monad`s quantum wave function .

The Inner Angular Momentum of Particles (L) as Spin (S) becomes of two Equal and perpendicular velocity Vectors $\overline{\mathbf{v x}}, \mathbf{v y}$, which travel Isochronal between nodes in the tiny Quantum-volume ds $=N / 2=(\mathrm{wr})^{2}$ .The centrifugal velocity vectors $\overline{\mathbf{V}} \mathbf{x}(\mathrm{r}), \overline{\mathbf{V y}}(\mathrm{r})$ as forces and the equal opposite on Evolute $-\overline{\mathbf{V}}(\mathrm{r}),-\overline{\mathbf{V y}}(\mathrm{r})$ create the Inner Electromagnetic wave $\mathbf{E}, \mathbf{P}$ in $\mathrm{NN}=2(\mathrm{wr})^{2}=2 \cdot \mathrm{s}^{2}=$ Vector breakage and as the single Dynamic standing wave structure E,P in $\mathrm{NN}= \pm(\mathrm{wr})^{2}= \pm \mathrm{s}^{2}=$ Scalar breakage $\rightarrow$ resonant. The wavelengths $\lambda=2$.NN of the two perpendicular Inward waves contain NN axis. The tangential velocity vectors $\overline{\mathbf{V x}}(\mathrm{T}), \overline{\mathbf{V y}}(\mathrm{T})$ as forces , equilibrium the opposite $-\overline{\mathbf{V}} \mathbf{x}(\mathrm{T}),-\overline{\mathbf{V y}}(\mathrm{T})$ on the centrifugal axis of Cycloid-Evolute for the Stability of the system, while both two angular momentum, $\overline{\mathbf{S x}}, \overline{\mathbf{S y}}$ components, the two Spins, following the Cross-Product 
are then composed to vector $\overline{\mathbf{S}}=\mathbf{S p i n}=\overline{\mathbf{V}}(\mathrm{T}) \mathbf{x X X}$, in $\mathbf{N N}$ axis because Spin $=$ Torque $=$ The angular equivalence Force.

$\overline{\boldsymbol{S}}=[\mathrm{g} \cdot \sin \varphi] \cdot \rho=[\mathrm{g} \cdot \sin \varphi] \cdot[2 \mathrm{c} \sqrt{\mathrm{r}} / \mathrm{g}=2 \mathrm{c} \sqrt{\mathrm{rg}} \cdot \sin \varphi \ldots(\mathrm{s})$

i.e. Spin, as Energy $\bar{c}$, is dependent on velocity Position only, since $\bar{v}=\bar{c}$. Equation (s) Unify the Energy, Spin of ,c , and Space Cave of , r . [51] Inner Wave and Outer wave, as the Spin vector, are superimposed at breakage with opposite amplitudes. Because at $\mathrm{NN}=\lambda / 2$, and at nodes evolute changes phase $\Pi=180^{\circ}$ rotation , and this because of the two neighboring lobes which are in anti-phase with each other, then exists phase shift at the center on which simultaneously Spin creates rotation .

Wave amplitudes E,P are equal to $\sqrt{ } 2 .\left|(w r)^{2}\right|$ at nodes and $E=P=\sqrt{ } 2 .\left|(w r)^{2}\right| \cdot \sin (\pi / 2+\pi) \cdot \cos w t$ at middles Nodes.

Above Attributes of monad ,allows to transform the Inward wave to another Outward wave and obtain constructive interference with the proper phase relation and the whole space of breakage to become twisted up and stretched without limit .

Since spin $\mathrm{S}=\mathrm{E} / \mathrm{w}= \pm \overline{\mathrm{v}}$. $(\mathrm{wr})^{2} / \mathrm{w}=\overline{\mathrm{v}}$. $\mathrm{wr}^{2}$, then has only One value for scalar breakages $(\mathrm{h}=1 / 2)$, and since also $\mathrm{S}=\mathrm{E} / \mathrm{w}= \pm \overline{\mathrm{v}}$. $(2 \mathrm{wr})^{2} / \mathrm{w}=2 . \bar{v}$. $\mathrm{wr}^{2}$, then has only One Double-value for vector breakages $(\mathrm{h}=1)$.

Since Energy caves are versors then energy $E /(\pi \sqrt{ } 3)$ and $E=\sqrt{ } 3 . \pi .(h / 2 \pi) . w=\sqrt{ } 12[12+1] . h w=\sqrt{ } 32$ Spin for spin value $\mathrm{s}=1 / 2$.

b.. From the principle of Linear superposition, if two waves of equal amplitude are in-phase then they meet crest-to-crest and trough-to-trough and their amplitudes add to each other (the Constructive Interference) and the difference in distance they travel is an integer, $\mathrm{n}=$ order, of wavelength $\lambda \rightarrow[\mathrm{DDT}=\mathrm{n} \lambda]$ and if the two waves are out-of-phase then they meet crest-to-trough and have zero amplitudes which cancel each other (it is called the Destructive Interference ) and the difference in distance they travel must differ by an odd integer , n, of wavelength $\lambda / 2 \rightarrow[\mathrm{DDT}=(\mathrm{n}+1 / 2)$.

c.. Diffraction is determined by the ratio $N / \mathrm{d}=\lambda / \mathrm{R}$ and thus for more diffraction of the waves is needed longer wavelengths and smaller opening .

A standing wave is generated by the superposition of two waves with the same frequency and wavelength travelling in opposite direction .

The standing wave does not carry energy from one point to another but serves to store energy (associated) since there is no energy transferring away, with specific wavelengths, i.e. a moving monad stores Kinetic energy , $\mathrm{T}$, and Dynamic energy, $\mathrm{V}$, in the Stationary wavelength $\lambda$, by the inner varying curvature which changes either the angular velocity, $\mathrm{w}$, or frequency, $\mathrm{f}$, or velocity, $\mathrm{v}$.

In particles the two perpendicular standing waves travel in the same direction and carry Energy in $\rightarrow$

a) the cave of wavelength $\lambda=2 \pi R=4 \pi$ as an Electromagnetic wave, Isochrones, of equal phase and direction and in case of double energy quantity in constant cave $\lambda$, two crests are created and frequency is doubled.

b) Angular momentum, as Spins, Anti-spins, and from cross-product Resultant Spin as equivalence Force causes the outer Screw motion of particles .

Spin is created from the tangential inner velocity

energy carrier. Vibrations of the medium parallel and or perpendicular to the direction of the motion define the type of waves as Longitudinal and as Transverse.

d.. Diffraction is determined as a source on an unknown backround medium of propagation and is, a dense damper medium, for storing the energy Id= Material Geometry the Periodic System and Chemistry . 17 (p.c2). $(\mathrm{wAo})^{2}$ of a wave, Ao, in it, and then is transformed as energy $\mathrm{Id}=\rho \mathrm{r}^{2} \mathrm{c}^{3} 2 \lambda^{2}$. $\left[\varepsilon \mathrm{E}^{2}+\mu \mathrm{H}^{2}\right]$ in the electromagnetic field E,H .This tiny medium is the dipole Fragments medium $\left\{\mathrm{ds}=|\lambda|=\left|2 .(\overline{\mathrm{w}} \cdot \mathrm{r})^{2}\right|=\left|+(\overline{\mathrm{w}} \cdot \mathrm{r})^{2}\right| \leftrightarrow \mid-\right.$ $\left.(\overline{\mathrm{w}} \cdot \mathrm{r})^{2} \mid\right\}$, in Spaces $\left[\mathrm{V}=4\left(w^{2} r^{2}\right)^{3} 3 \pi\right.$ ] of Gravity`s medium [MFMF] field and consists the infinite tiny dipole volumes caves with their inner Transverse Electromagnetic field E,H and which are the tanks in where Energy of dark points and dark fringes of any two interference waves is stored . For all moving monads, Kinetic Energy $\mathrm{T}$ as Torque causes the Outer motion, and Dynamic energy V, causes the Inner Electromagnetic E,H field .

Electromagnetic waves are able to transmit Energy through a vacuum (empty space) by storing their energy in above Standing Transverse Electromagnetic dipole wave , and thus considered completely particle like, and in the transverse interference pattern tobe considered as completely wave, so the same quantity of energy behaves as, Energy $\mathrm{Id}=\rho \pi^{2} \mathrm{c}^{3} 2 \lambda^{2}\left[\varepsilon^{2}+\mu \mathrm{H}^{2}\right]$ in volume $\mathrm{V}=\left[4(w 2 r 2)^{3} 3 \pi\right]$ having mass $\rightarrow$ Particle Energy $I d=(\rho . c 2) .(w A o)^{2}$ in Interference pattern as $\rightarrow$ Wave This is the Wave-Particle duality unifying the classical Electromagnetic field and the quantum particle of light . Spin $=\mathbf{E w}=\left[ \pm \overline{\mathrm{v}} \cdot \mathrm{s}^{2}\right] / \mathrm{w}=\left(\mathrm{r} \cdot \mathrm{s}^{2}\right)=$ $\mathrm{w}^{2} \mathrm{r}^{3}=[\mathrm{wr}]^{3}$, or $\rightarrow \mathbf{h} \boldsymbol{\pi}=2 .[\mathrm{wr}]^{3}$, or Energy Space quantity wr, is doubled and becomes the Space quantity $\mathbf{h} \boldsymbol{\pi}$

The above relation of Spin shows the deep relation between Mechanics and E-geometry, where the tiny Gravity-cave in $\mathrm{r}=10-62 \mathrm{~m}$, as the Energy- Volume - quantity [wr] , is doubled and is Quantized in Planck`s-cave Space quantity as, $(\mathrm{h} / \pi)=\operatorname{Spin}=2 .[\mathrm{wr}]^{3}$ in $\mathrm{r}=10-35 \mathrm{~m}$ i .e. Energy Space quantity, wr , is 
Quantized, and becomes the New Space quantity, $\mathbf{h} / \mathbf{\pi}=\mathbf{2} \cdot[\mathbf{w r}]^{\mathbf{3}}$, doubled , following the Euclidean Spacemould of Duplication of the cube, in tiny Sphere volume $V=(4 \pi / 3)$. [wr/2 $]^{3}$, as well as that of Squaring of circle , $\mathbf{\pi}$, and or in Sub-Space-Sphere volume ${ }^{3} \sqrt{ } \mathbf{2}$.

Since $w=E /[h / 2 \pi]=m \cdot c^{2} /[h / 2 \pi]=2 \pi \cdot m c^{2} / h=2 r \cdot s^{2}=2 \cdot r^{3} \cdot w^{2}$, then mass $\mathbf{m}=(w r)^{3} c^{2}=2 c^{2}(w r)^{3}$, and is what is called conversion factor mass , $\mathbf{m}$, as an index of energy changes .

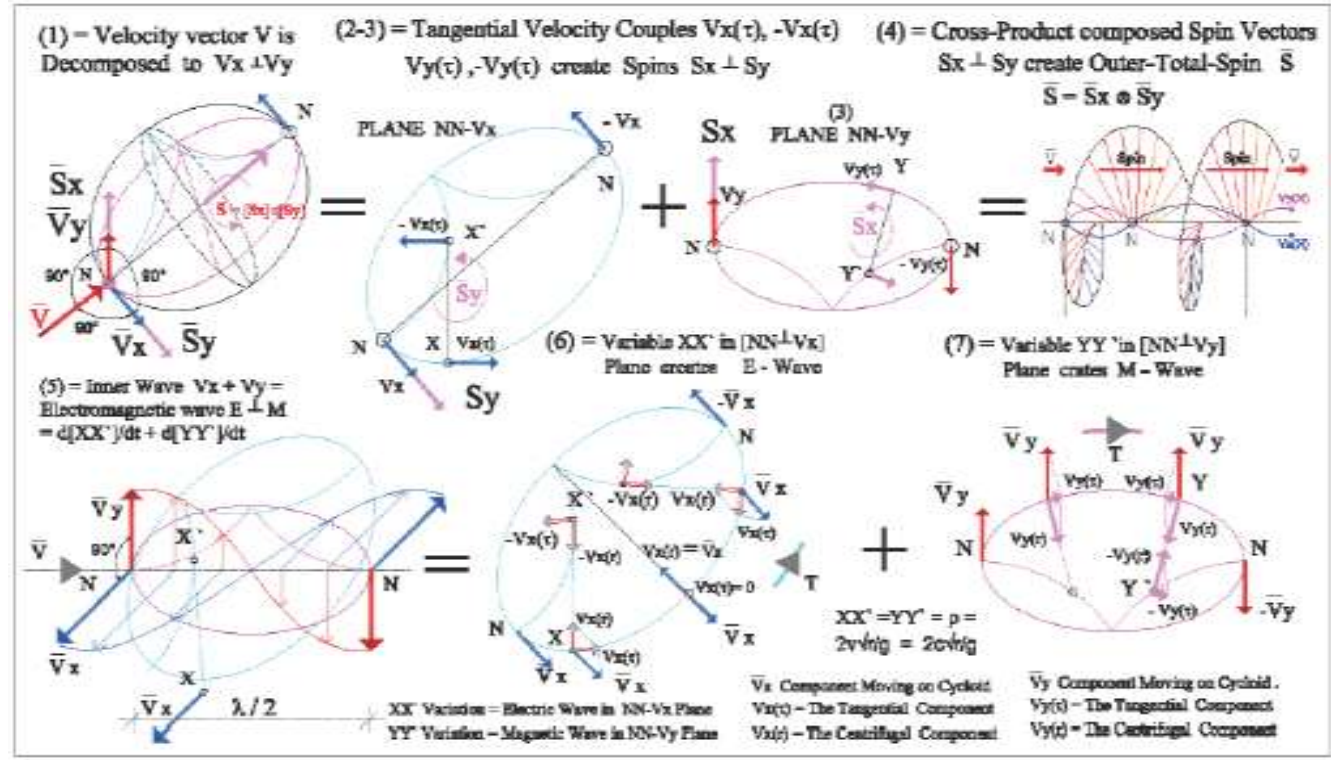

Figure. 5.

The Mechanism of Angular Momentum - Spin - in Particles and their Curl-motion . The Collision between velocity vector $\bar{v}$ and a Unit $N N$, produces the two transverse Electromagnetic-Waves and Spin as Torque, which creates the motion of Unit .

Outer Spin of Particles creates E,P Inner Waves and in tern Inner Spin as Torque and again, is converted to External Spin and particle moves, thus Recycled.

\section{Analyses :}

Figure .5

In $(\mathbf{5}, \mathbf{5})$, Energy as velocity vector $\overline{\mathbf{V}}$ at node $\mathbf{N}$, following the Cross-product, is decomposed into the two perpendicular components $\overline{\mathrm{V}} \mathrm{x}, \overline{\mathrm{V}} \mathrm{y}$, which consist the Electromagnetic wave in breakages $\left( \pm \mathrm{s}^{2}, 2 \mathrm{~s}^{2}=N / 2\right)$ being a Quantum Space Resonance. The component Velocities are retarded, because of the Isochrones motion on breakage`s cycloid.

In (5,2-3), Transverse Velocity components $\overline{\mathrm{V}} \mathrm{x} \quad, \overline{\mathrm{V}} \mathrm{y}$ in point $\quad, X, Y$, are also decomposed to the Centrifugal Velocities $\bar{V} x(r), \bar{V} y(r)$ on radius of curvature $r=X^{\prime}$ or $r=Y^{\prime}$ where $X^{\prime}, Y^{\prime}$ is the Curvature center on evolute, and to transverse Tangential Velocities $\overline{\mathrm{V}} \mathrm{x}(\mathrm{T}), \overline{\mathrm{V}} \mathrm{y}(\mathrm{T})$, to cycloid .Tangential velocity couples $\overline{\mathrm{V}} \mathrm{x}(\mathrm{T}),-\overline{\mathrm{V}} \mathrm{x}(\mathrm{T})$ and $\overline{\mathrm{V}} \mathrm{y}(\mathrm{T}),-\overline{\mathrm{V}} \mathrm{y}(\mathrm{T})$ create spins Sx, Sy on $\overline{\mathrm{V}} \mathrm{y}, \overline{\mathrm{V}} \mathrm{x}$ direction . The Plane system of $\overline{\mathbf{S x}}, \overline{\mathrm{Sy}}$,

vectors following the cross product, is composed to vector $\overline{\boldsymbol{S}}$ acting on breakage and because lever arm $\mathrm{XX}^{\prime}, \mathrm{YY}^{`}$ is varying, Resultant Momentum execute an Outer, varying Curl-Whirling motion and thus produces the motion of breakage as in (4).

In (5, 6-7) Since, in any action there is an equal and opposite reaction, motion on transverse Cycloids equilibrium on opposite evolute motion, therefore exists the opposite velocity couples $\bar{V} x(r),-\bar{V} x(r)$ and $\bar{V} y(r)$,$\overline{\mathrm{V}} \mathrm{y}(\mathrm{r})$, collinear and of opposite direction on , the varying Space-arms XX',YY'.

The system is Stable and is in equilibrium because forces cancel each other, and Because arms $\mathbf{X X}^{\prime}$, YY` are varying, therefore, since velocity vectors $\mathrm{d}\left(\mathrm{XX}^{`}\right) / \mathrm{dt}, \mathrm{d}\left(\mathrm{YY}^{`}\right) / \mathrm{dt}$, in Inner curvature between Cycloid-Evolute are shifting and create oscillation, where dt the varying repetitive position, executing a harmonic motion, and thus are produced the Electric and the Magnetic field of monads .

The magnetic force is a Torque as an Inertial force to the direction of motion. For gravity level $r$ $=10-62 \mathrm{~m}$ and $\mathrm{r}^{3}=10-186 \mathrm{~m}$, the relative strength is not detected . For Planck `s level $\mathrm{r}=10-35 \mathrm{~m}$ where $\mathrm{r}^{3}=10-105 \mathrm{~m}$, this is the detected and measurable Spin .Considering Planck`s length $\mathrm{L}=1,616.10-35 \mathrm{~m}$ filled with the two only breakages $s^{2}= \pm\left|(\overline{\mathrm{w}} . \mathrm{r})^{2}\right|$ then $(1) \leftrightarrow(2)=\mathrm{L}=\lambda=2 .\left|(\overline{\mathrm{w}} \cdot \mathrm{r})^{2}\right|$. Using Planck`s Work equations connecting particles and wave properties $\mathrm{W}=\Lambda \cdot \mathrm{ds}=\mathrm{p} \cdot \Lambda=\mathrm{h}$ then $\mathrm{p}=\mathrm{h} \lambda$ and from Kinetic energy $\mathrm{E}=\mathrm{mv}^{2} / 2=$

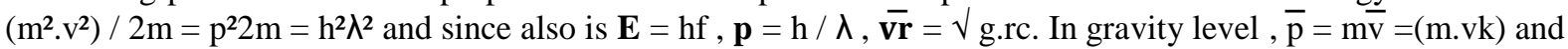


$|\bar{p}|^{2}=|m \cdot v k|^{2}=m^{2} \cdot g \cdot r c$ and $E=p^{2} 2 m=m \cdot g \cdot r 2=h^{2} \lambda^{2}$, where $r=r c$ For Planck`s length then $\lambda=1,616 \cdot 10-35$ and constant velocity $\mathrm{c}=2,9979$. 108 then Period is $, \mathrm{T}=N \mathrm{c}=5,3867 . .10-44 \mathrm{~s}$ and frequency $\mathrm{w}=2 \pi \mathrm{T}=1,16.1044$ cycle/sec.

For a material point, electron, $\lambda=1,616.10-9 \mathrm{~m}$ it is $\mathrm{mg} \cdot \mathrm{r} 2=\mathrm{h}^{2} \lambda^{2}=[6,626.10-34]^{2} /[1,616.10-9]^{2}=$ $1,681.10-49$ and for $\mathrm{g}=9,81 \mathrm{~m} / \mathrm{s}^{2}$ then $\mathrm{r}=1,681 \cdot 10-49 \cdot(2 / 9,81) \mathrm{m}=3,427.10-50 \mathrm{~m}$, which is the rolling circle`s radius in electron wavelength .

For a material point under Planck`s length then $\lambda=\mathrm{L} / 2=2 \pi \mathrm{r}$ and $\mathrm{r}=\mathrm{L} / 4 \pi=1,616.10-35 / 4 \pi=$ 1,286.10-36 m .

Kinetic energy $E=m v^{2} / 2=h . f=h / T=h /(4 \pi r / v)=h v /(4 \pi r)$ and since also $p=h / \lambda=h /(L / 2)=2 h / L$ then $p=$ $2(6,626.10-34) /(1,616.10-35)=82$.

From energy relation $\mathrm{p}=\mathrm{h} /(2 \pi \mathrm{r})=\mathrm{m} \cdot \mathrm{v}=\mathrm{m} \sqrt{\mathrm{gr}}$ or, $\mathrm{h}^{2}=4 \pi^{2} \mathrm{r}^{2}$. $(\mathrm{g} \cdot \mathrm{r}) \cdot \mathrm{m}^{2}=4 \pi^{2} \mathrm{r}^{3} \mathrm{~g} \cdot \mathrm{m}^{2}$ then $\mathrm{m}^{2}=\mathrm{h}^{2} /\left[4 \pi^{2} \mathrm{r}^{3} \mathrm{~g}\right]$ $=(6,626.10-34)^{2} /\left[4 \pi^{2} \cdot(1,286.10-36)^{3} \cdot 9,81\right]=(4,26410-4.10-68) / 10-111=5,3303 \cdot 1038$ and $\mathrm{m}=$ $2,3067.1019 \mathrm{Kg}$.

$\mathrm{m} / \mathrm{s}$

From constancy of $\overline{\mathrm{v}} \mathrm{k}=\sqrt{\mathrm{gr}}$ then $\mathrm{v}^{2}=(9,81.1,286.10-36)=12,61566.10-36$ and $\rightarrow \overline{\mathrm{v}} \mathrm{k}=3,552.10-18$

Period $\mathrm{T}=\lambda / \overline{\mathrm{v}} \mathrm{k}=(1,616.10-35) /(2.3,552.10-18)=2,275.10-18 \mathrm{~s}$ Frequency $\mathrm{f}=1 / \mathrm{T}=1 /(2,275.10-18)=$ 4,396.10-17 Momentum $\mathrm{p}=\mathrm{m} . \mathrm{v}=2,3067.1019 .2,9979.108=6,915.1027 \mathrm{Kgm} / \mathrm{s}$. From energy equations Energy $\mathrm{E}=\mathrm{p}^{2} / 2 \mathrm{~m}=(6,915.1027)^{2} /(2.2,3067.1019)=1,0364.1036 \mathrm{Kgm}$. Considering Planck `s length $\mathrm{L}=1,61.10-35 \mathrm{~m}$ filled with infinite breakages $\mathrm{s}^{2}= \pm\left|(\overline{\mathrm{W}} \cdot \mathrm{r})^{2}\right|$ then $(1) \leftrightarrow(2)=\lambda=2 .\left|(\overline{\mathrm{W}} \cdot \mathrm{r})^{2}\right|$. These tiny energy volumes caves (cycloids in $\lambda$ ) consist in gravity`s field, the infinite tanks in where energy of dark points and dark fringes of any two interference waves is conserved.

Stability is obtained by the inner and opposite rotational momentum in Anti-cycloid motion .Radiation of Energy is enclosed in a cavity of the tiny energy volume $\lambda==\left[\frac{4\left(w^{2} r^{2}\right)^{3}}{3 \pi}\right]$, (cycloidal) with perfect and reflecting boundaries and this cavity may become infinite in every direction and thus Material Geometry the Periodic System and Chemistry . 19 getting in maxima cases (the limits) the properties of radiation in free space. The electromagnetic vibrations in this volume is analogous to vibrations happening in an Elastic body (the stresses) into this volume and thus Fringes are a superposition of the standing vibrations.

This Energy radiation system of particles maybe represented in Space by the three states of interaction or and Internal-external positions ,

a).. The position of the particle by means of any system to general coordinates or in Cartesian coordinates ,

b).. The state of radiation field is determined by the values of the components of the Electric and Magnetic vectors at points of the space, or the field by means of a scalar and a vector potential (the values of scalar potential) and,

c)..The action of the Particle as a whole on this inside Field (the coupling energy of the inner constraints due to evolute ).

\section{Spin And Torque}

In [49] was shown that equation of angular velocity $\mathrm{w}=\mathrm{E} / \mathrm{h} / 2 \mathrm{~T}]$ and defines the number of notes that will be present along the medium, the cave, and is depended upon the Incident Energy ,E, on cave so , w = $\mathrm{E} /[\mathrm{h} / 2 \pi]=\mathbf{E} \cdot[2 \pi / \mathrm{h}]=\mathbf{E} / \mathbf{S P I N}$ When the applied force $\mathbf{E}=\mathrm{hf}=\mathbf{w} \cdot(\mathrm{h} / 2 \pi)=\mathrm{w}$. Spin then $\mathbf{S p i n}=\left[\frac{\mathbf{E}}{\mathbf{w}}\right]=$ $\left[ \pm \bar{v} \cdot s^{2}\right] / w=\left(r . s^{2}\right)$ on cave and are created Fermions with $\operatorname{Spin}=\left[\frac{E}{w}\right]=\left(\mathbf{r} \cdot s^{2}\right) \rightarrow\left[\frac{E}{w}\right]$ When the applied force $\mathbf{E}$ $=\mathrm{h} \cdot \mathrm{f}=\mathbf{w} \cdot(\mathrm{h} / 2 \pi)=\left[\nabla \mathrm{i}=2(\mathrm{wr})^{2}=2 \cdot \overline{\mathrm{v}} \mathrm{s}^{2}\right]$ on cave then $\mathbf{S p i n}=\left[\frac{\mathbf{E}}{\mathbf{w}}\right]=\left[2 \cdot \overline{\mathrm{v}} \cdot \mathrm{s}^{2}\right] / \mathrm{w}=2 .\left(\mathrm{r} \cdot \mathrm{s}^{2}\right)$ and are created Bosons with $\operatorname{Spin}=\left[\frac{E}{w}\right]=2 .\left(r . s^{2}\right) \rightarrow 2 .\left(\frac{E}{w}\right)=1$ which is double the before. i.e.

Energy as velocity vector $\overline{\mathrm{v}}$ or $\mathbf{E}=[ \pm \overline{\mathrm{v}}]$, applied on the material energy dipole points $\left[ \pm \mathrm{s}^{2}\right]$ as the Dipole Quantity $|\overline{\mathrm{v}}|^{2}=|\overline{\mathrm{w}} \times \overline{\mathrm{r}}|^{2}=|\overline{\mathrm{w}} \cdot \mathrm{r}|^{2}=\mathrm{s}^{2}$, separately creates the massive Particles, Fermions, while the velocity vector $\overline{\mathrm{v}}$, applied on the Double Quantity Energy vector breakages $\nabla \mathrm{i}=2(\mathrm{wr})^{2}=2 \cdot \mathrm{s}^{2}=2[ \pm \overline{\mathrm{v}}]^{2}=\mathbf{2 E}$ and since $\mathrm{v}=$ (w.r), $2 \mathrm{v} \cdot \mathrm{s}^{2}=2|\overline{\mathrm{v}}| \mathrm{s}^{2}=2 \cdot|\overline{\mathrm{w}} \times \overline{\mathrm{r}}|^{3}=2 \mathrm{v} \cdot|\overline{\mathrm{w}} \cdot \mathrm{r}|^{2}=2 \mathrm{~s}^{2}$ creates the Energy Particles, the Bosons, by doubling its frequency [ f $2=2 . \mathrm{f} 1]$ in the same cave.

Energy is the Cause of motion, the change of position of points, and it is motion itself . Mass is the Reaction to the motion, it is an Index of the amount of motion, and a numeric magnitude which has nothing to do with Energy .

In mechanics -Physics, Change, is that of Space, ds, and it is the velocity vector $\overline{\mathbf{v}}$, that of change of velocity vector and it is acceleration $\mathbf{d} \overline{\mathbf{v}}$, that of change of the Reaction to the Velocity and Direction of the motion and it is mass $\mathbf{m}$.

All Units = monads have their place in Spaces . The Second-order differential equation excited by a Harmonic external force Ft sin wt is as , $\mathrm{m} \mathrm{d}^{2} \mathrm{xdt}^{2}+\mathrm{c} d \mathrm{xdt}+\mathrm{k}$. $\mathrm{x}=\mathrm{Ft} \sin \mathrm{wt}$ corresponds physically to the free 
damped vibration, where $\mathrm{x}=$ the displacement, $\mathrm{dx} / \mathrm{dt}=$ the velocity and $\mathrm{d}^{2} \mathrm{x} / \mathrm{dt}^{2}=$ the acceleration of monad, and $\mathrm{m}, \mathrm{c}, \mathrm{k}$ constants, with general solution given by the equation $\mathrm{x}=\mathrm{A} \cdot e^{s 1 \cdot t}+\mathrm{B} \cdot e^{s 2 \cdot t}+\mathrm{X} \sin (\mathrm{wt}-\varphi) \ldots \ldots(1)$ In Electromagnetism, Change, say a Space-monad is $\rightarrow$ a Resonance which can occur in the RLC circuit , where Resistance $\mathbf{R}$, is the change in current amount it is the converter of current, Inductance $\mathbf{L}$, is like mass or Inertia in Mechanical systems which store the Magnetic-energy and, Capacitance $\mathbf{C}$, concentrates $( \pm)$ charge which store the Electric-energy in much the same way that springs store mechanical energy inverse spring constant, is the analogous .

The differential equation excited by a Harmonic Electromotive force Et sin wt, in an RLC circuit , oscillating at its natural frequency is as, $\mathrm{L} \mathrm{d}^{2} \mathrm{qdt}^{2}+\mathrm{R} \mathrm{dqdt}+\frac{1}{C} \mathrm{q}=\mathrm{Et}$ sin wtcorresponds physically to the free damped vibration, where Charge $\mathrm{q}=$ is the physical property of matter that causes it to experience a force which can be positive or negative, $\mathrm{dq} / \mathrm{dt}=$ the least quantized amount of charge and $\mathrm{d}^{2} \mathrm{q} / \mathrm{dt}^{2}=$ the space distribution of charge , and L , R , C Inductance , Resistance , Elastance = Elasticity constants , with general solution given by the equation $\mathrm{q}=\mathrm{A} \cdot e^{s 1 . t}+\mathrm{B} \cdot e^{s 2 \cdot t}+\mathrm{X} \sin (\mathrm{wt}-\varphi) \ldots . .(2)$

Equations (1) and (2) give the analogic relation of the Classical mechanics [Space position , $\mathrm{x}$,] and the Electromagnetism [Quanta of energy ,q,] of Storing and Removing of energy in Energy-Space cosmos .

In E-Geometry -Mechanics, Change, say monad $\mathrm{NN}$ is an cycloidal Resonance $\mathrm{NN}=N / 2=2 \pi \mathrm{r}=\mathrm{s}^{2}$ $=[2 \pi \mathrm{w}]^{2}=4 \pi \mathrm{V} / \mathrm{c}$, clashed with velocity vector $\overline{\mathrm{v}}=\overline{\mathrm{c}}$, and causing velocity components $\mathrm{V}(\mathrm{x}), \mathrm{V}(\mathrm{y})$ which move forth and back, up and down, and thus forced to Vibrate at a specific fundamental frequency .[49] The Material Geometry and the Origin of Energy-Particles in Periodic System 20 Motion is as the Charge in Physics , and occurs from the Cross - product velocity components $|\bar{v} x|,|\bar{v}| y$ where $|\bar{v}|^{2}=|\bar{v} x| \mathbf{x}|\bar{v}| y$ validating in clashed inherent vibration particles .

Potential Energy is as the Voltage and occurs from Cross-product velocity $\mathrm{Vx}$, Vy couplecomponents $\mathrm{Vx}(\mathrm{r}),-\mathrm{Vx}(\mathrm{r})$ on radius of curvature $\rho=4 \operatorname{rcos} \varphi$ and from the transverse centrifugal velocity couple $\mathrm{Vy}(\mathrm{r}),-\mathrm{Vy}(\mathrm{r})$ the magnetic Voltage .

Since $\rho=X^{\prime}=4 r \cdot \cos \varphi$ and is varying on Cycloid -Evolute, which is Space Anti-space equilibrium , this creates oscillation in $\left[\mathrm{Vx}(\mathrm{r}), \mathrm{XX}^{\prime}\right]$ plane and thus producing Electric field. The same also for transverse varying $Y^{`}$ creating Magnetic field in [Vy(r),YY] Plane . Oscillation is so produced from the varying Space Positions XX', YY', forth and back -up and down, and this because of Geometry-Mechanics relation .

Spin is the Resultant of Plane system of $\overline{\mathbf{S}} \mathrm{X}, \overline{\mathbf{S}} \mathrm{y}$, vectors into Vy , Vx axis which follows the Cross-product , and is composed to vector $\square$ acting on breakage NN axis as a Torque , and because lever arms XX', YY are varying, Resultant Momentum execute an Outer, Whirling motion producing the Outer Oscillating motion of breakage.

Following Newton`s 1st and 3rd law for Spin at Nods N,N where Spin is swept, monad = N/2 is Push forward as velocity vector following the same In-cycle by forming the Outward Electromagnetic field .

In Figure 4-5, Space $s^{2}=\left|(\mathrm{wr})^{2}\right|=\mathrm{ds}$ is the tiny Energy resonance between NN nodes so, any Changes to Motion Correspond to both, Classical mechanics and Electromagnetism equations .

In figure. 5-1, the Changeable Radius of Curvature ,formed between Cycloid and Anti-cycloid is $X^{\prime}=\rho=$ 4.r. $\cos \varphi$ which depends on angle $\varphi$, is following a cosine`s curve, and it is at N,N points, where for $\varphi=90^{\circ}$, then $\rightarrow \rho=0$ and for the extrema case at $\varphi=270^{\circ}$, then $\rightarrow \rho=2 \pi \cdot r$, and simultaneously $\rho=0$. This changeable, Radius of Curvature, creates the varying cycloidal Electromagnetic wave of monads which is Regenerated between N,N nods, as a kind of geometrical variation position .

In figure.5-2, the Changeable Tangential Velocity $\overline{\mathrm{V}} \mathrm{x}(\mathrm{T})=\mathrm{c} \cdot \sin \varphi$ depends on angle $\varphi$, following a sinus curve , it is at $\mathrm{N}, \mathrm{N}$ points, where $\varphi=90^{\circ}$ and $\varphi$

$=270^{\circ}, \rightarrow \mathrm{Vx}(\mathrm{T})=\mathrm{c}$ and at middle points $\mathrm{O}, \mathrm{O}^{\prime}$, where $\varphi=0^{\circ}, \rightarrow \mathrm{Vx}(\mathrm{T})=0$. It is continually equilibrium with the opposite velocity $-\overline{\mathrm{V}} \mathrm{x}(\mathrm{T})$.

In figure.5-3 the Changeable, Tangential Velocity

$\overline{\mathrm{V}} \mathrm{x}(\mathrm{r})$, creates Spin vector $\overline{\mathrm{S}}=2 \mathrm{rc} \cdot \sin 2 \varphi=4 \mathrm{rc} \cdot \sin \varphi \cdot \cos \varphi$, depending on angle $\varphi$, following the sinus curve $2 \varphi$, and instantly at $\mathrm{N}, \mathrm{N}$ points, where $\varphi=90^{\circ}$ and $\varphi=270^{\circ}$, , which is the extrema case $\rightarrow \overline{\mathrm{S}}=2 \pi \mathrm{r} . \mathrm{c}$, and after this immediately becomes zero until angle $\varphi=2 \pi / 5$ accepting the maximum value $\rightarrow \bar{S}=2$ r.c. At middle points $\mathrm{O}, \mathrm{O}^{\prime}$, where $\varphi=0^{\circ}$, then $\operatorname{spin} \overline{\mathrm{S}}=0$ even if $\rho=4 \mathrm{r}$.

Since $\mathbf{S p i n}=$ Torque $=[\overline{\mathbf{S}} \mathrm{x}] \mathrm{x}[\overline{\mathbf{S}} \mathrm{y}]=\mathrm{Vx}(\mathrm{T}) \cdot \rho \cdot \sin \varphi=$ the Angular equivalence of Force $=$ Torque in NN axis of monad $\{\mathrm{NN}=$ a moving or not frame with magnitude $=$ scalar $=|\mathrm{S}|$, and direction that of axis of vector $\overline{\mathrm{S}}\}$, and which force never vanishes, therefore is conserved .

By this vanishing velocity, Spin is swept away from monad as a kind of, General Clearance, for the New regenerated Electromagnetic field $E \perp P$ by the centrifugal velocity vectors $\bar{V} x(r), \bar{V} y(r)$.

Meanwhile Total Torque $|\vec{S}|=2 \pi r . c$, exists in NN axis, so monad NN would still be moving, this per Newton`s first law of motion, and this is according to conservation of , Total Impulse, and rotating due to conservation of , Linear and angular momentum. The compound Centrifugal Force due to the two $\operatorname{Vx}(\mathrm{T})$,- 
$\mathrm{Vx}(\mathrm{T}), \mathrm{Vy}(\mathrm{T}),-\mathrm{Vy}(\mathrm{T})$ is a centripetal force making monad to move in a circle or a helix as equation $\mathrm{C}-\mathrm{F}=\mathrm{mv} \mathbf{x} \mathrm{w}$ $=-(\mathrm{wr})^{2} . \mathrm{vxH}=-(\mathrm{wr})^{3} \mathbf{x H}$.

According to math theory of Elasticity, the total work on free edges where there is no shear becomes from Principal stresses only and it is $\mathrm{W}=\sigma^{2} / 2 \mathrm{E}+\tau^{2} / 2 \mathrm{G}$ and the analogous Energy in monads $\mathrm{W}=12\left[\varepsilon \mathrm{E}^{2}+\mu \mathrm{H}^{2}\right]$ spread as the

First Harmonic and equal to Spin $\bar{S}=2 \pi r . c$. Planck`s Energy $E=h . f=(h / \lambda) . c$ is equal to the Isochromatic pattern fringe-order in monad as

$\sigma 1-\sigma 2=(\mathrm{a} / \mathrm{d}) \cdot \mathrm{N}=(\mathrm{a} / \mathrm{d}) \cdot \mathrm{n} \cdot \mathrm{f} 1=\left(8 \pi \mathrm{r}^{2} / 3\right) \cdot \mathrm{n} \cdot \mathrm{f} 1$

and the Summation of their Isochromatic

Quantized fringe-order is $\mathrm{E}=\overline{\mathrm{S}}=2 \pi \mathrm{r} . \mathrm{C}=$

$\left[\frac{8 \pi r^{2} \mathrm{f} 1}{3}\right] \cdot\left[\frac{n(n+1)}{2}\right]=\left[\frac{4 \pi r^{2} \mathrm{f} 1}{3}\right] \mathrm{n} \cdot(\mathrm{n}+1)$ of the same cave $(\mathrm{wr})^{2}$.

When stress $(\sigma 1-\sigma 2)$ go up then ,

$\mathbf{n}=$ order fringe defining Energy goes up also , and the colors cycle through a more or less repeating pattern and the Intensity of the colors diminishes . For $n=1$, the First Harmonic , $E=2 \pi r . c=\left[4 \pi \pi^{2} 3\right]$.f1.[1] , and for

$\mathrm{n}=2$ the Second Isochromatic fringe Quantized order , $\mathrm{n}$, as threes and, $\mathrm{E}=\left[4 \pi^{2} 3\right] . \mathrm{f} 1 \rightarrow \varphi$ trisection with Energy-Bunched variation $\mathrm{f} 2=2 \mathrm{f} 1$.

This is the way of Energy storing in caves (wr) ${ }^{2}$.

\section{Remarks :}

a.. From definitions, Quantum-Spaces, or , Quantum-Monads are the Scalars $\left[ \pm s^{2}= \pm(\mathrm{wr})^{2}\right]$

and Vector $\left[\nabla \mathrm{i}=2\right.$.(wr) $\left.{ }^{2}\right]$ breakages all quantized

through [STPL] E-geometry [SS] Mould .

Thrust is , Energy applied on breakages as velocity vector $\overline{\mathrm{v}}$.

Wave-Structures, are the clashed homogeneously breakages, with the velocity vector $\overline{\mathrm{v}}=\overline{\mathrm{c}}$.

Spin is fixed and independent of particle`s mass

because Spin $=(\mathrm{h} / 2 \pi)$. W , or its external angular

velocity, $\mathbf{w}$, is monad`s quantum wave function .

The Inner Angular Momentum of Particles (L) as Spin (S) becomes of two Equal and perpendicular velocity Vectors $\overline{\mathbf{v}} \mathbf{x}, \overline{\mathbf{v}} \mathbf{y}$, which travel Isochronal between nodes in the tiny Quantum-volume ds $=N / 2=(\text { wr })^{2}$. The centrifugal velocity vectors $\overline{\mathbf{v}} \mathbf{x}(\mathrm{r}), \overline{\mathbf{v}} \mathbf{y}(\mathrm{r})$ as forces and the equal opposite on Evolute $-\overline{\mathbf{v}} \mathbf{x} \overline{\mathbf{v}}(\mathrm{r}),-\overline{\mathbf{v}} \mathbf{y}(\mathrm{r})$ create the Inner Electromagnetic wave $\mathbf{E}, \mathbf{P}$ in $\mathrm{NN}=2(\mathrm{wr})^{2}=2 . \mathrm{s}^{2}=$ Vector breakage and as the single Dynamic standing wave Vibrating structure $E, P$ in

$\mathrm{NN}= \pm(\mathrm{wr})^{2}= \pm \mathrm{s}^{2}=$ Scalar breakage $\rightarrow$ resonant. The wavelengths $\lambda=2 . \mathrm{NN}$ of the two perpendicular Inward waves contain NN axis .

The tangential velocity vectors $\overline{\mathbf{V}} \mathbf{x}(\mathrm{T}), \overline{\mathbf{V}} \mathbf{y}(\mathrm{T})$ as forces, equilibrium the opposite $-\overline{\mathbf{V}} \mathbf{x}(\mathrm{T}),-\overline{\mathbf{v}} \mathbf{y}(\mathrm{T})$ on the centrifugal axis of Cycloid-Evolute for the Stability of the system, while both two angular momentum, $\overline{\mathbf{v}} \mathbf{x}, \overline{\mathbf{v}} \mathbf{y}$ components, the two Spins, following the Cross-Product are then composed to vector $\overline{\mathbf{S}}=\mathbf{S} \mathbf{S p i n}=\overline{\mathbf{V}} \mathbf{x}(\mathrm{T}) \mathbf{x}$ $\mathrm{XX}^{\prime}$, in $\mathbf{N N}$ axis because $\mathrm{Spin}=$ Torque $=$ The angular equivalence Force.

$\overline{\mathbf{S}}=[\mathrm{g} \cdot \sin \varphi] \cdot \rho=[\mathrm{g} \cdot \sin \varphi] \cdot[2 \mathrm{c} \sqrt{\mathrm{r}} / \mathrm{g}=2 \mathrm{c} \sqrt{\mathrm{rg}} \cdot \sin \varphi . .(\mathrm{s})$

i.e. Spin , as Energy $\square$, is dependent on velocity Position only, since $\bar{v}=\bar{c}$. Equation (s) Unify the Energy , Spin of ,c , and Space Cave of ,r . [51]

Inner Vibrating Wave and Outer Oscillating wave, as the Spin vector, are superimposed at breakage with opposite amplitudes .

Because at $\mathrm{NN}=\lambda / 2$, and at nodes evolute changes phase $\pi=180^{\circ}$ rotation, and this because of the two neighboring lobes which are in anti-phase with each other, then exists phase shift at the center on which simultaneously Spin creates rotation . [49]

Wave amplitudes E,P are equal to $\sqrt{ } 2 .\left|(\mathrm{wr})^{2}\right|$ at

nodes and $\mathrm{E}=\mathrm{P}=\sqrt{2} \cdot\left|(\mathrm{wr})^{2}\right| \cdot \sin (\pi / 2+\pi) \cdot \cos w t$ at

middles Nodes.

Above Attributes of monad ,allows to transform the Inward wave to another Outward wave and obtain constructive interference with the proper phase relation and the whole space of breakage to become twisted up and stretched without limit .

Since spin $\mathrm{S}=\mathrm{E} / \mathrm{w}= \pm \overline{\mathrm{v}}$. $(\mathrm{wr})^{2} / \mathrm{w}=\overline{\mathrm{v}}$. $\mathrm{wr}^{2}$, then has only One value for scalar breakages $(\mathrm{h}=1 / 2)$, and since also $\mathrm{S}=\mathrm{E} / \mathrm{w}= \pm \overline{\mathrm{v}} \cdot(2 \mathrm{wr})^{2} / \mathrm{w}=2 . \overline{\mathrm{v}} \cdot \mathrm{wr}^{2}$, then has only One Double-value for vector breakages $(\mathrm{h}=1)$.

Since Energy caves are versors then energy $E /(\pi \sqrt{ } 3)$ and $E=\sqrt{ } 3 . \pi \cdot(h / 2 \pi) . w=\sqrt{ } 12[12+1] . h w=\sqrt{ } 32$ Spin for spin value $s=1 / 2$. 
b.. From the principle of Linear superposition, if two waves of equal amplitude are in-phase then they meet crest-to-crest and trough-to-trough and their amplitudes add to each other (the Constructive Interference) and the difference in distance they travel is an integer, $n=$ order, of wavelength $\lambda \rightarrow$ [DDT $=n \lambda]$ and if the two waves are out-of-phase then they meet crest-to-trough and have zero amplitudes which cancel each other (it is called the Destructive Interference ) and the difference in distance they travel must differ by an odd integer , $n$, of wavelength $N / 2 \rightarrow[\mathrm{DDT}=(\mathrm{n}+1 / 2) \cdot \lambda]$

For all moving monads, Kinetic Energy $\mathrm{T}$ as Torque causes the Outer motion, and Dynamic energy V, causes the Inner Electromagnetic E,H field .

Electromagnetic waves are able to transmit Energy through a vacuum (empty space) by storing their energy in above Standing Transverse Electromagnetic dipole wave, and thus considered completely particle like, and in the transverse interference pattern to be considered as completely wave, so the same quantity of energy behaves as ,

Energy $\mathrm{Id}=\rho \mathrm{r}^{2} \mathrm{c}^{3} 2 \lambda^{2}\left[\varepsilon \mathrm{E}^{2}+\mu \mathrm{H}^{2}\right]$ in volume $\mathrm{V}=\left[\frac{4\left(w^{2} r^{2}\right)^{3}}{3 \pi}\right]$ having mass $\rightarrow$ Particle Energy

$\mathrm{Id}=($ o.c2 $) .(\mathrm{wAo})^{2}$ in Interference pattern as $\rightarrow$ Wave

This is the Wave-Particle duality unifying the classical Electromagnetic field and the quantum particle of light .

$\operatorname{Spin}=\frac{\mathbf{E}}{\mathbf{w}}=\left[ \pm \overline{\mathrm{V}} \cdot \mathrm{s}^{2}\right] / \mathrm{w}=\left(\mathrm{r} \cdot \mathrm{s}^{2}\right)=\mathrm{w}^{2} \mathrm{r}^{3}=[\mathrm{wr}]^{3}$, or $\rightarrow$

$\frac{E}{w}=2 \cdot[w r]^{3}$, or Energy Space quantity wr , is doubled and becomes the Space quantity $\frac{h}{\pi}=(h / \pi)$

The above relation of Spin shows the deep relation between Mechanics and E-geometry, where the tiny Gravity-cave in $\mathrm{r}=10-62 \mathrm{~m}$, as the Energy- Volume - quantity [wr] , is doubled and is Quantized in Planck`s-cave Space quantity as,$(\mathrm{h} / \pi)=\mathrm{Spin}=2$. $[\mathrm{wr}]^{3}$ in $\mathrm{r}=10-35 \mathrm{~m}$ i .e.

Energy Space quantity ,wr , is Quantized, and becomes the New Space quantity, $\mathbf{h} / \mathbf{m}=\mathbf{2}$.[wr] ${ }^{3}$, doubled, following the Euclidean Space-mould of Duplication of the cube, in tiny Sphere volume $\mathrm{V}=$ $(4 \pi / 3)$. $[\mathrm{wr} / 2]^{3}$, as well as that of Squaring of circle , $\boldsymbol{\pi}$, and or in Sub-Space-Sphere volume ${ }^{3} \sqrt{ } \mathbf{2}$

Since $\mathrm{w}=\mathrm{E} /[\mathrm{h} / 2 \pi]=\mathrm{m} \cdot \mathrm{c}^{2} /[\mathrm{h} / 2 \pi]=2 \pi \cdot \mathrm{mc}^{2} / \mathrm{h}=2 \mathrm{r} \cdot \mathrm{s}^{2}=2 \cdot \mathrm{r}^{3} \cdot \mathrm{w}^{2}$, then mass $\mathbf{m}=(\mathrm{wr})^{3} \mathrm{c}^{2}=2 \mathrm{c}^{2}(\mathrm{wr})^{3}$, and is what is called conversion factor mass , $\mathbf{m}$,

as an index of energy changes .

\section{Particles And Momentum}

In general, momentum $\mathrm{p}=\mathrm{m} \bar{v}$, therefore the amount of momentum per unit volume, $\mathrm{dV}$, is $, \rho \bar{v},,($ since $\mathrm{m}=$ volume . $\rho)$ and for surface, the normal stress $\sigma=\mathrm{F} / \mathrm{dS}=-\mathrm{F}$ and then becomes $\rightarrow \partial \rho \bar{v} / \partial \mathrm{t}+\nabla \cdot(\rho \cdot \mathrm{d} \bar{v}) . \mathrm{V}=\nabla . \sigma$ $+\rho . g$, i.e. momentum is converted into a normal stress,$\sigma$, in tiny volume plus momentum of the whole volume as mass , body force, where $\nabla$ is nabla symbol for, divergency, $\mathrm{g}$, is gravity`s net acceleration, , $\rho$, the inner volume density. This interpretation applied to Young`s interference and Polarizer experiments for dark fringes then $\rho=\left[\varepsilon \mathrm{E}^{2}+\mu \mathrm{H}^{2}\right]$ and thus is shown the unified understanding between the classical Electromagnetic field theory and the quantum particle of light. The maximum velocity occurs in Common circle , when the two velocities , $\overline{\mathrm{c}}, \overline{\mathrm{v}}$ are perpendicular between them, from where then dispersion follows Pythagoras theorem and the resultant Quantized linear Space length ,r, becomes , as the Resultant of Energy Vectors ,

$\mathrm{r}=|(\overline{\mathrm{c}} . \mathrm{T})|=\sqrt{\mathrm{v}^{2}+\mathrm{c}^{2}} . \rightarrow[52]$

Using Space Vector $\mathrm{r}=|(\overline{\mathrm{c} . T})|=\sqrt{\mathrm{v}^{2}+\mathrm{c}^{2}}$ then

The total Rotating energy is $\rightarrow \pm \Lambda=\bar{p} \cdot r=$

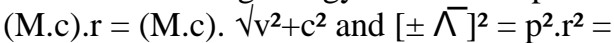

$\mathrm{M}^{2} \cdot \mathrm{c}^{2} \cdot\left(\mathrm{v}^{2}+\mathrm{c}^{2}\right)=\left(\mathrm{M}^{2} \cdot \mathrm{v}^{2}\right) \cdot \mathrm{c}^{2}+\mathrm{M}^{2} \cdot \mathrm{c} 4=\left(\mathrm{p}^{2} \cdot \mathrm{c}^{2}\right)+$

$\mathrm{M}^{2} . \mathrm{c} 4=[\mathrm{p} . \mathrm{c}]^{2}+\left[\text { mo. } \mathrm{c}^{2}\right]^{2}$

which is the known equation from Relativity and because this property of axis of rotation becomes from the Spinning-Wheel-Rim and assuming any Orientation exists only on Gyroscope-Mechanism, where The three RIM-Frames, with one and two degrees of rotational freedom are tilting rotation of mounting according to Rotating-Energy axis.

\section{Definition Of Material Geometry}

In [39-41] was shown the geometrical Mechanism [STPL] $=$ [Six-Triple-Points-Line] where energy

Massive and Vector Breakages , $\left[ \pm s^{2}= \pm(w r)^{2}\right]$ and $\left[\nabla i=2(w r)^{2}\right]$, become the Materials .

Action (@) of a quaternion $\overline{\mathrm{z}}=\mathrm{s}+\overline{\mathrm{v}} \mathrm{i} .=\mathrm{s}+\overline{\mathrm{v}} \cdot \nabla \mathrm{i}$ on itself is the Binomial type as ,

$(\mathrm{s}+\overline{\mathrm{v}} \cdot \nabla \mathrm{i})(\mathrm{C})(\mathrm{s}+\overline{\mathrm{v}} \cdot \nabla \mathrm{i})=[\mathrm{s}+\overline{\mathrm{v}} \cdot \nabla \mathrm{i}]^{2}=\mathrm{s}^{2}+|\overline{\mathrm{v}}|^{2} \cdot \nabla \mathrm{i}^{2}$

$+2|\mathrm{~s}| \cdot \overline{\mathrm{v}} \cdot \nabla \mathrm{i}=\mathrm{s}^{2}-|\overline{\mathrm{v}}|^{2}+2|\mathrm{~s}| \cdot|\overline{\mathrm{w}} \cdot \mathrm{r}| \cdot \nabla \mathrm{i}=$

$\mathrm{s}^{2}-\mathrm{s}^{2}+[2 \overline{\mathrm{w}} \cdot \mathrm{r}] \cdot|\mathrm{s}| \cdot \nabla \mathrm{i}=\mathrm{s}^{2}-\mathrm{s}^{2}+2 . \mathrm{s}^{2}$ where, 
$\mathrm{s}^{2}=(\mathrm{wr})^{2} \rightarrow$ is the real part of the new quaternion and it is a Positive -Space and Scalar magnitude. $-\mathrm{s}^{2}=-|\overline{\mathrm{w}} \mathrm{r}|^{2} \rightarrow$ the always Negative-Anti-space which is always a Negative Scalar magnitude.

$2 . s^{2}=[2 \overline{\mathrm{w}}] .|\mathrm{s}| .|\mathrm{r}| . \nabla \mathrm{i} \rightarrow$ the double angular velocity term which is a Vector magnitude.

In the recovery equilibrium (a surface of a cylinder with $2 \mathrm{r}$ diameter), and because velocity vector is on the circumference while in the center axis is zero, the infinite breakages Identify with points A,B,C (of the extreme triangles $\mathrm{ABC}$ of Space $\mathrm{ABC}$ ) and with points $\mathrm{AE}, \mathrm{BE}, \mathrm{CE}$, of the extreme triangles of Anti-space $\mathrm{AE}$ $\mathrm{BE} \mathrm{CE}$.

Points AE,BE,CE of Anti-Space are all on the same circumference of the prior formulation and are rotated with the same angular velocity vector $\overline{\mathrm{w}}$. The inversely directionally rotated Energy $\pm \Lambda$ equilibrium into the common circle, so Spaces and Anti-Spaces meet in this circle which is the common Sub-space . Extreme Spaces (the Extreme triangles ABC) meet Anti-Spaces (the Extreme triangles of AEBE CE ), through the only Gateway which is the Plane Geometrical Formulation Mechanism (mould) of the [STPL] line, or as a cylinder . [17]

The $\rightarrow$ [ Space, Anti-Space equilibrium,$\pm \Lambda^{-}$, Absolute System $\left.[\mathrm{S}] \leftarrow\right]$, as Angular momentum $\Lambda^{-} \Omega$ $=$ m.v.r , is Crushed out into Fragments and, becoming the three Breakages $\left[\mathrm{s}^{2}=(\mathrm{wr})^{2}\right],\left[-\mathrm{s}^{2}=-(\mathrm{wr})^{2}\right],[\nabla \mathbf{i}$ $=2(\mathrm{wr})^{2}$. $]$, and after clashed with the velocity vector $\bar{v}$ of $[\mathrm{S}]$ system, (unless succeed to escape un-clashed through center $\mathrm{O}$ in [STPL] line and this because $\overline{\mathrm{v}}=0$ ), are Thrown OFF this System [S], conveyed into the Linear momentum , the Inertial and Energy -Space , which is the Relative [STPL] System [ R] and are the Particles Fermions $\rightarrow[ \pm \overline{\mathrm{v}} . \mathrm{s}]$ and Bosons $\rightarrow[\overline{\mathrm{v}} . \nabla \mathrm{i}]$.

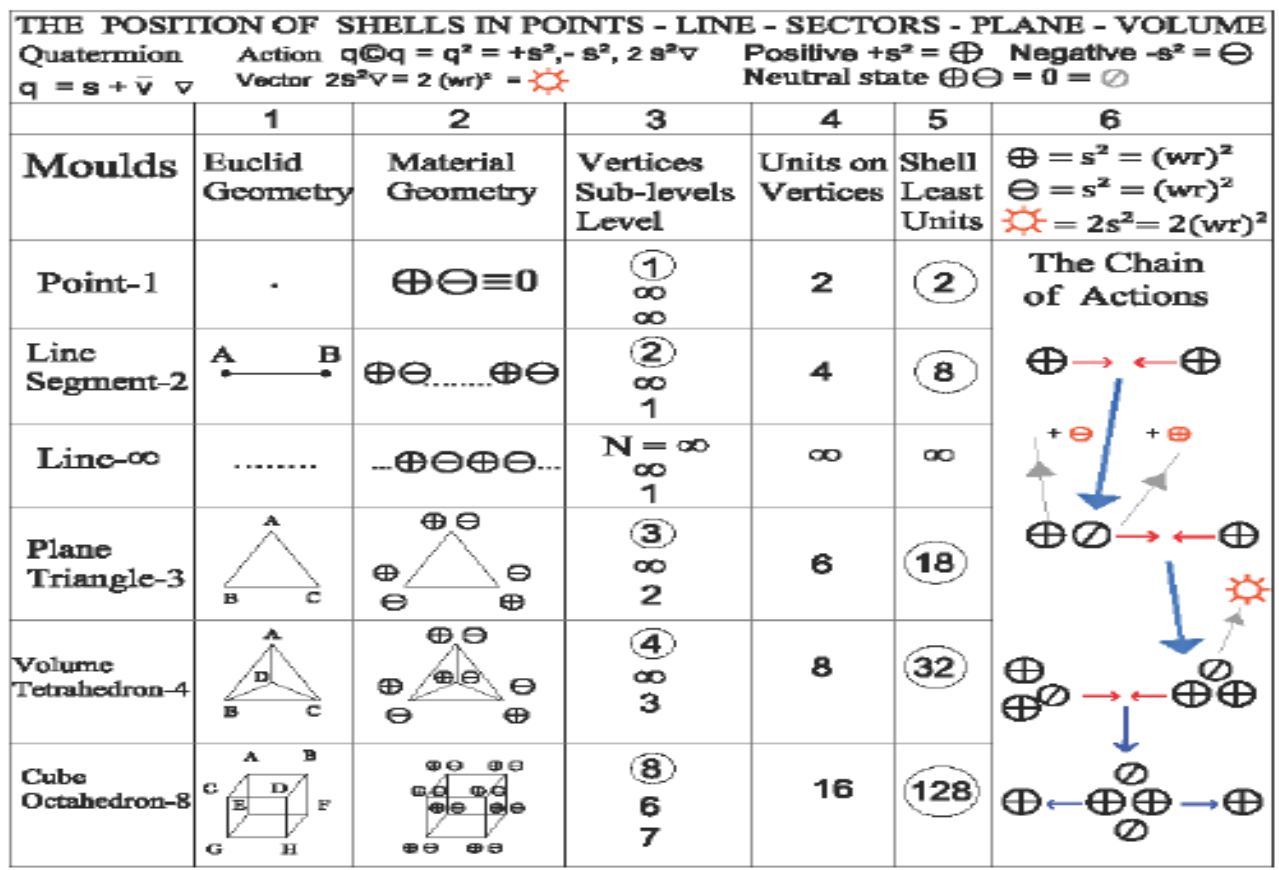

Figure.6.

The Euclidean Geometry ,EG, moulds and the corresponding to Material Geometry ,MG. Actions for Quaternion, Collision for Particles, happen between Units on and in, Line Segments, Planes, Volumes and Spaces by following Rules of E-geometry .

In [1] The Elements of E-Geometry $\rightarrow$ Point (.) ,Line Segment AB (-), Plane triangle ABC, Volume Tetrahedron ABCD and Spaces Cube ABCDEFGH are Extrema.

In [2] The maximum, Extrema, Elements in M-Geometry $\rightarrow$ Point 2 , Line Segment 4 , Plane 6 , Volume 8 , and $\mathrm{N}$ Spaces $2 \mathrm{~N}$, Cube 16 .

In [3] The minimum number for Vertices /Sub-levels /Level, needed in each Level $\equiv$ Mould.

In [4] The maximum number, Extrema, of Units in Vertices of monads ,in Points ,of Moulds.

In [5] The SLU, Shell-Least Monad-Unit-Number, or the Permissive Sites within Premises

Levels and the maximum number of Sites for Sub-levels ,in each M-Geometry Element which is , Point $\rightarrow 2$ ,Line segment $\rightarrow 8$,Plane $\rightarrow 18$,Volume $\rightarrow 32, \mathbf{m}$ Space $\rightarrow 2 \mathbf{m}^{2}$.

SLU is $\rightarrow$ Full of Outer Permissive, Equilibrium Sites, and with Low Reactivity,

In [6] The Main branch of one, of the Chain of Actions, in Planck`s Level .

Un-clashed Fragments through center O , consist the \{ Medium-Field Material-Fragment $\}$ 
$\rightarrow\left[ \pm \mathrm{s}^{2}\right]=[\mathrm{MFMF}]$ as base for all motions , and Gravity as force $[\nabla \mathrm{i}]$, while the clashed with the constant velocity, $\overline{\mathrm{c}}$, consist the Dark matter

$[ \pm \bar{c} . s]$ and the Dark energy $[\bar{c} . \square \mathrm{i}]$, or from Breakages $\left[ \pm \mathrm{s}^{2}= \pm(\mathrm{wr})^{2}\right],\left[\nabla \mathrm{i}=2(\mathrm{wr})^{2}\right]$ become

A.. $\left[+\overline{\mathrm{V}} . \mathrm{s}^{2}\right] \rightarrow$ Fermions, through $[$ STPL]

B.. $\left[-\bar{v} . S^{2}\right] \rightarrow$ Anti-Fermions ,through [STPL]

C.. $[\uparrow \bar{v} . \nabla \mathrm{i}] \rightarrow$ Bosons , through [STPL]

D.. $[\downarrow \overline{\mathrm{v}} . \nabla \mathrm{i}] \rightarrow$ Anti-Bosons , through [STPL]

E.. $\left[ \pm \mathrm{s}^{2}\right] \rightarrow[\mathrm{MFMF}]$ Field , through $\mathrm{O}$

F.. $[\nabla \mathrm{i}] \rightarrow$ Gravity Field , through $\mathrm{O}$

G.. $[\overline{\mathrm{v}}( \pm) \nabla \mathrm{i}] \rightarrow$ Gravity Force, through $\mathrm{O}$

H.. $\left[ \pm \bar{c} . \mathrm{s}^{2}\right] \rightarrow$ Dark matter , through O

I.. $[\overline{\mathrm{c}}( \pm) \nabla \mathrm{i}] \rightarrow$ Dark energy , through $\mathrm{O}$

Thrust $(\overline{\mathrm{v}}=\overline{\mathrm{w}} \cdot \mathrm{r})$ of $\left[\right.$ STPL] Mechanism is continually acting on the Breakages $\left[\mathrm{s}^{2},-|\overline{\mathrm{v}}|^{2},[2 \overline{\mathrm{w}}] \cdot|\mathrm{s}||\mathrm{r}|=\right.$ $\left.2(\overline{\mathrm{w}} \cdot \mathrm{r})^{2}\right]$ producing the $\left.[1-1+2] \cdot \overline{\mathrm{w}}^{3} \cdot \mathrm{r}\right|^{3}$ magnitudes $(\mathrm{w} \cdot \mathrm{r})^{3}$, which is a Positive Scalar magnitude, with Positive or zero electric charge and called Spin , $1 / 2$ or $1=[2 \bar{w}] \cdot|s| \cdot|\mathrm{r}| \cdot \nabla \cdot[43-49]$

1.. Positive breakage Quantity $|\overline{\mathrm{v}}|^{2}=|\overline{\mathrm{w}} \mathrm{xr}|^{2}=|\overline{\mathrm{w}} . \mathrm{r}|^{2} \rightarrow$ Being at Space points A,B,C of Common-Circle mechanism and the Action magnitudes Q at the coinciding points DADB DC -PAPB PC of it, Produces Leptons and Quarks , and carry them on [STPL] line. [30-36]

2.. Negative breakage Quantity $-|\overline{\mathrm{v}}|^{2}=-|\overline{\mathrm{w}} \mathrm{xr}|^{2}=-|\overline{\mathrm{w}} \cdot \mathrm{r}|^{2} \rightarrow$ Being at Space points A,B,C then Action magnitudes $\mathrm{Q}$ at coinciding points DA,DB ,DC -

PA,PB,PC Produces Anti-Leptons and Anti-Quarks, and carry them on [STPL] line.

3.. Positive breakage Quantity $[2 \overline{\mathrm{w}} \mid] \cdot|\mathrm{s}| \cdot|\mathrm{r}| \cdot \square \mathrm{i}=2 \mathrm{w} .(\mathrm{sr}) \cdot \nabla \mathrm{i}=2 \mathrm{w} \cdot\left(\mathrm{r}^{2} \cdot \mathrm{w}\right) \cdot \nabla \mathrm{i}=2 \mathrm{w} \cdot \mathrm{r}^{2} \mathrm{w} \cdot \nabla \mathrm{i} \rightarrow$ Bosons, being at Space points A,B,C of Common-Circle mechanism and then Action magnitudes $Q$ at coinciding points $D_{A}, D_{B}$, $, \mathrm{D}_{\mathrm{C}},-\mathrm{P}_{\mathrm{A}}, \mathrm{P}_{\mathrm{B}}, \mathrm{P}_{\mathrm{C}}$, Produces Bosons , and carry them on [STPL] line.

4.. Breakage Quantities $\left[ \pm \mathrm{s}^{2}= \pm(\mathrm{wr})^{2}\right]$, being at, $\mathrm{O}$, commons` circle center and shacked OFF into [STPL] , and this because of $\mathrm{v}=0$, formulate the [MFMF] Field, which consist the base of all motions .

5.. Breakage Quantities $\nabla \mathrm{i}=2(\mathrm{wr})^{2}$ ] being at , O, commons circle center and shacked OFF into [STPL] , and this because of $v=0$, formulate the Gravity Field and Force in [MFMF] Field .

6. Breakage Quantities $\left[ \pm \overline{\mathrm{c}} . \mathrm{s}^{2}\right]$ and $[\overline{\mathrm{c}} . \nabla \mathrm{i}]$, the clashed with the constant velocity $\overline{, \mathrm{c}}$, formulate in [STPL] cylinder Dark matter and Dark Energy respectively in [MFMF] Field . i.e.

It was above referred that Thrust $[\overline{\mathrm{c}} . \square \mathrm{i}]$ moves with light velocity and is continually acting on the five EnergySpace Fragments as $\rightarrow$

$[\bar{c} . \nabla \mathrm{i}] \odot\left\{(\nabla \mathrm{i}),\left(+\mathrm{s}^{2}\right),\left(-\mathrm{s}^{2}\right),\left(+\mathrm{cs}^{2}\right),\left(-\mathrm{cs}^{2}\right)\right\}$ and on the two Dipole-Couples, where for the Rest -Couple [ $(\nabla \mathrm{i}) \rightarrow\left(+\mathrm{s}^{2}\right) \leftrightarrow\left(-\mathrm{s}^{2}\right)=$ It is the Gravity Thrust acting on the Stationary dipole creating the Rest Gravity-Field and Force] , and for Moving -Couple [ $(\square \mathrm{i}) \rightarrow\left(+\mathrm{cs}^{2}\right) \leftrightarrow\left(-\mathrm{cs}^{2}\right)=$ It is the Gravity Thrust which is acting on the light velocity moving dipole and is creating in it the Rest Gravity -Field and Force dipole Dark

-Matter Energy-Field ] . i.e. The same force for the two Couples.

The two , opposite signed , Fragments $\mathrm{s}^{2}= \pm\left|(\overline{\mathrm{w}} \cdot \mathrm{r})^{2}\right|$ consist the under Gravity primary Dipole , [ $\left.\left|+\mathrm{s}^{2}\right| \leftrightarrow\left|-\mathrm{s}^{2}\right|\right]=|\lambda| \equiv\left[\left|+(\overline{\mathrm{w}} \cdot \mathrm{r})^{2}\right| \leftrightarrow\left|-(\overline{\mathrm{w}} \cdot \mathrm{r})^{2}\right|\right]$ where on this, Force $\left[\nabla \mathrm{i}=2 . \mathrm{s}^{2}=2(\mathrm{wr})^{2}\right]$ as velocity $\overline{\mathrm{v}}=\overline{\mathrm{c}}$, the Thrust , causes the Gravity`s Electromagnetic Field $\mathrm{E} \perp \mathrm{P} \rightarrow[\nabla \mathrm{i}] .\left(+\mathrm{s}^{2}\right),[\nabla \mathrm{i}] .\left(-\mathrm{s}^{2}\right),[\nabla \mathrm{i}] \cdot\left|\left( \pm \mathrm{s}^{2}\right)\right|=$

$[\nabla \mathrm{i}] .\left[\left|+\mathrm{s}^{2}\right| \leftrightarrow\left|-\mathrm{s}^{2}\right|\right]$, while Thrust $(\nabla \mathrm{i})$ acting on $\left( \pm \mathrm{cs}^{2}\right)$ dipole $\left[(\nabla \mathrm{i}) \rightarrow\left(+\mathrm{cs}^{2}\right) \leftrightarrow\left(-\mathrm{cs}^{2}\right)\right]$ creates the Gravity $\rightarrow$ Darkmatter Energy-field .[40-43]

The massive particles Fermions and Bosons :

Velocity vector $\bar{v}$ acting on breakages $\pm \mathrm{s}^{2}=(\mathrm{wr})^{2}$ is creating Fermions and Anti-fermions, while vector $\overline{\mathrm{v}}$ acting on vector $\nabla \mathrm{i}=2 .(\mathrm{wr})^{2}$ creates Boson, Anti-bosons . In Figure.3, Breakages $\left[ \pm \mathrm{s}^{2}= \pm(\mathrm{wr})^{2}\right]$ occupying the minimum Space, $\pi(\mathrm{wr}) 66 \rightarrow$ is Volume of the tiny cave of radius $r,\left[\varepsilon \mathrm{E}^{2}+\mu \mathrm{H}^{2}\right] \rightarrow$ is the Density of the tiny cave and Material Geometry the Periodic System and Chemistry .

mass $\rightarrow . \nabla \cdot \nabla=\mathbf{V} \cdot \mathbf{\rho}=\pi(\mathrm{wr}) 66\left[\varepsilon \mathrm{E}^{2}+\mu \mathrm{B}^{2}\right]$

In Common-circle Cylinder $N N=N 1 N 2=\left[ \pm \mathrm{s}^{2}\right]=|\overline{\mathrm{w}} \mathrm{xr}|^{2}$ the tiny Energy-Space is following the trajectory, in $=(\mathrm{N} 1)$, or , out $=(\mathrm{N} 2)$, Cycloid $=(\mathrm{c})=|\mathrm{N} 1-\mathrm{N} 2|=[\{\mathrm{N}(\mathrm{P} 1) \leftarrow 0 \rightarrow(\mathrm{P} 2) \mathrm{N}\}]$ and needs more or less time $\mathrm{T}(2)<\mathrm{T}=4 \pi \sqrt{(\mathrm{r} / \mathrm{g})}<\mathrm{T}(1)$ to reach the other end point N2 . [33-36]

And since frequency $\mathrm{f}=1 / \mathrm{T}$ and energy $\mathrm{E}=\mathrm{h}$.f then Cycloid motion Controls constancy of Energy, the inner stability of monad, by changing velocity, $\overline{\mathrm{v}}=\overline{\mathrm{w}} \cdot \mathrm{r}=2 \pi \mathrm{r} / \mathrm{T}=2 \pi \mathrm{r}$.f , and period, $\mathrm{T}$, of stationary monad as , $\mathrm{E}=$ h.f $=$ h. $(\bar{v} / 2 \pi r)$. 
Breakage quantity 2.(wr) $)^{2}$ under the tangential action $\overline{\mathrm{v}}=$ wr becomes 2.(wr) $)^{3}$ acting on point $\mathrm{A} \rightarrow$ 2 wr.m of common circle. The same also for points A,B,C of Space and AE,BE,CE of Anti-Space . Because all velocity vectors AA, BB , CC carry the material points A,B,C at points DA ,DB , DC in time ,t, isochrones , then material points follow a cycloid with period the norm of wavelength of velocities $|\mathrm{AA}|,|\mathrm{BB}|,|\mathrm{CC}|$.

This Simultaneity is succeeded by Lorentz factor where transformations between Inertial frames that preserve the velocity of light will not preserve simultaneously. The Geometrical expression of this transformation (it is the base Mould STPL, of the natural Universe ) follows i.e.

Through [ STPL ] line, the mould, which is the Primary Particle Mould, Energy as velocity vector is quantized to $\rightarrow$ Particles -Anti-particles , Gravity-Field and Gravity-Force , Dark matter and Dark energy , and all material world , following Cycloidal simultaneity • [17,31,35,41]

It was referred what is point, sector and line in Euclidean geometry. The Material point (1) at Euclidean point (1), is now Breakage $\pm\left[(\overline{\mathrm{w}} \cdot \mathrm{r})^{2}\right]$ dipole volume which becomes from that cave where quantized Point length = $\mathrm{Lv}=\mathrm{r}=$ ei.(N.T2). $\mathrm{b}=10^{-} \mathrm{N}$, and for $\mathrm{N}=\infty \rightarrow \mathrm{Lv}=0$.

It is one of the two equal and opposite equilibrium basic elements $\left(\left|+(\overline{\mathrm{W}} \cdot \mathrm{r})^{2}\right| \leftrightarrow\left|-(\overline{\mathrm{W}} \cdot \mathrm{r})^{2}\right|\right)$ in this Rest Homogenously and Isotropically Quantized, mass-less Field [PNS] in the spherical volume V $=\square 6 .\left[\left| \pm(\overline{\mathrm{W}} \cdot \mathrm{r})^{2}\right|\right]$ ${ }^{3}$, which consist in our Planck`s confinement the required coordinate System and the base for all motions and forces in Gravity parallel Inertial systems . This rest element in Space system [PNS] (is the Base ) into the Medium-Field Material Fragment $\rightarrow[\mathrm{MFMF}]$ Field with the less space distance of diameter, $\mathrm{ds}=|\overline{\mathrm{w}} \cdot \overline{\mathrm{r}}|^{2}$, which consists the Minimum Space material point and Energy Quanta.

It was referred that Fragments $\mathrm{s}^{2}= \pm\left|(\overline{\mathrm{W}} . \mathrm{r})^{2}\right|$ occupying the minimum quantized space $\left|\mathrm{s}^{2}\right|$ are deported and fill all [STPL] cylinder which is the Rest Quantized Field $\pm\left[(\overline{\mathrm{w}} \cdot \mathrm{r})^{2}\right]$ or it is , the material point in mechanics , and the base of all motions where Force $\left[(\overline{\mathrm{w}} \cdot \mathrm{r})^{2} \nabla \mathrm{i}\right]=2 \cdot\left[(\overline{\mathrm{w}} \cdot \mathrm{r})^{2}\right]=2 . \mathrm{s}^{2}$ is vibrating on length $2 .\left|\left[(\overline{\mathrm{w}} \cdot \mathrm{r})^{2}\right]\right|=\lambda$ as a Stationary Wave, and creates the curl Electromagnetic Field , E $\perp$ P , on which is the Universal Quantized force called Gravity . The Gravity -Force is equal to $\mathrm{Fg}=|\overline{\mathrm{q}}| \cdot[\mathrm{E}+\overline{\mathrm{v}} \mathrm{xP}]$ and is exerted on any movable particle with charge $\overline{\mathrm{q}}$. Gravity - Field $\mathrm{Gf}=[\mathrm{E}+\overline{\mathrm{v} x P}]$, is the un-movable, forced welded spinning dipole , $\rightarrow\left[\left|+(\overline{\mathrm{w}} \cdot \mathrm{r})^{2}\right| \leftrightarrow\left|-(\overline{\mathrm{w}} \cdot \mathrm{r})^{2}\right|=|\lambda|\right]=[\{\mathrm{A}(\mathrm{PA}) \leftarrow 0 \rightarrow(\mathrm{PB}) \mathrm{B}\}] \leftarrow$ and because above is movable then with three Unis becomes the un-movable Torsion $\pm \Lambda$ as ,

$\rightarrow\left[\left|(-\overline{\mathrm{W}} \cdot \mathrm{r})^{2}\right| \leftrightarrow\left|+(\overline{\mathrm{W}} \cdot \mathrm{r})^{2}\right| \leftrightarrow\left|-(\overline{\mathrm{W}} \cdot \mathrm{r})^{2}\right|\right]=|\Lambda| \equiv$ Spin $\longleftarrow$ and also because jointed with forces, this means that Newton`s laws issue in both, Absolute System [S] and to the Relative System [R].

The Material wavelength of two Units (1),(2), or the sector $\lambda=(1)-(2)$ or the minimum energy Space of the two material Units as above is as $\rightarrow\{$ [ Medium-Field Material Fragment $] \rightarrow\left[ \pm \mathrm{s}^{2}\right]= \pm|\overline{\mathrm{w}} \cdot \overline{\mathrm{r}}|^{2}=[\mathrm{MFMF}]$ Field $\leftarrow\}$ the Electromagnetic Field $\quad[\overline{\mathrm{E}} \times \overline{\mathrm{H}}]$ or the field $\rightarrow \quad\left[\left|+(\overline{\mathrm{W}} \cdot \mathrm{r})^{2}\right| \leftrightarrow\left|-(\overline{\mathrm{W}} \cdot \mathrm{r})^{2}\right|=|\lambda|\right] \equiv[\{(1)(\mathrm{J}=\mathrm{P} 1) \leftarrow 0 \rightarrow$ $(\mathrm{J}=\mathrm{P} 2)(2)\}] \leftarrow$ which is the Standing wave in cavity (1)-(2), of the two scalar breakage $\left| \pm(\overline{\mathrm{w}} \cdot \mathrm{r})^{2}\right|$ as medium (1)(2) field , and (J1) =2 $|\overline{\mathrm{w}} \cdot \mathbf{r}|^{2}$ as energy at point (1) and carried to point (2) by following the cycloid motion from (1) to (2) with an isochrones Velocity , $\bar{v}$, and during shifting is analyzed into two velocity vectors $\overline{\mathrm{v}} 1, \overline{\mathrm{v}} 2$, and which undergo vibrations causing two waves that represent the two, Electric E and Magnetic H, perpendicular components following the trajectory, $\mathrm{in}=(\mathrm{c} 1)$, out $=(\mathrm{c} 2)$. On cycloid $=(\mathrm{c})=|(1)-(2)|$ is needed the isochrones time $\mathrm{T}=2 \pi \mathrm{r} \cdot \mathrm{r} / \mathrm{g}$ to reach end (2) < Fermat's Principle of Least time > and this because it is the Extreme < Isochrones Principle > which is embedded in all wavelength vector monads, and consists the Minimum Energydipole-Quanta i.e. the sector (1)-(2). [39-41]

The Material wavelength of three Units (2)(1)(2), or the sector $\lambda=(2)-(1)-(2)$ is the Electromagnetic Field $[\overline{\mathrm{E}} \times \overline{\mathrm{H}}]$ or the Rest field as,$\rightarrow\left[\left|(-\overline{\mathrm{w}} \cdot \mathrm{r})^{2}\right| \leftrightarrow\left|+(\overline{\mathrm{w}} \cdot \mathrm{r})^{2}\right| \leftrightarrow\left|-(\overline{\mathrm{w}} \cdot \mathrm{r})^{2}\right|\right]=|\Lambda| \equiv$ Spin $\leftarrow$ The Material Geometry and the Origin of Energy-Particles in Periodic System

$[(1)(\mathrm{J}=\mathrm{P} 1) \rightarrow(\mathrm{J}=\mathrm{P} 2)(2)] \equiv[\square \leftarrow(\mathrm{J}=\mathrm{P} 2)(2)] \equiv\{\square \leftrightarrow \square\}$

which is the Reciprocating-motion,

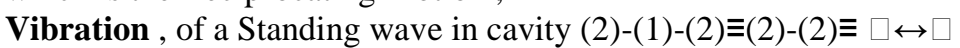

For Dark-matter , the Material point (1) at Euclidean point (1), is now the moving Breakage $\left[ \pm \overline{\mathrm{c}} . \mathrm{s}^{2}\right]$ volume which becomes from that cave where quantized Point $=\mathrm{Lv}=\mathrm{r}=$ ei.(N.T2). $\mathrm{b}=10^{-} \mathrm{N}=\infty$, infinite, and it is the movable element in the Rest Homogenous and Isotropically Quantized, mass-less Field [PNS] in the same spherical volume

$\mathrm{V}=\square 6\left[ \pm \overline{\mathrm{c}} \cdot \mathrm{s}^{2}\right]^{3}$.

For Dark-matter wavelength of two points (1),(2), or the sector $\lambda=(1)-(2)$ or the minimum energy Space of two Dark -matter material points is energy field $\left[ \pm \overline{\mathrm{c}} \cdot \mathrm{s}^{2}\right]=\left[\mathrm{c} .|\overline{\mathrm{w}} \cdot \overline{\mathrm{r}}|^{2}\right]=\left[\left|+\mathrm{c} .(\overline{\mathrm{w}} \cdot \mathrm{r})^{2}\right| \leftrightarrow\left|-\mathrm{c} .(\overline{\mathrm{w}} \cdot \mathrm{r})^{2}\right|=[\mathrm{c} .|\lambda|] \leftarrow\right.$ or the DM massive energy field . Force $\left[(\overline{\mathrm{w}} \cdot \mathrm{r})^{2} \cdot \nabla \mathrm{i}\right]=2 \cdot\left[(\overline{\mathrm{w}} \cdot \mathrm{r})^{2}\right]$ is also vibrating on same length $\left| \pm \overline{\mathrm{c}} \cdot \mathrm{s}^{2}\right|=$ $2\left|\left[\mathrm{c} .(\overline{\mathrm{w}} \cdot \mathrm{r})^{2}\right]\right|=\lambda$ as the Stationary Wave of gravity , and creates the curl Dark -Matter Electromagnetic Field [ $\overline{\mathrm{E}} \mathrm{x} \overline{\mathrm{H}}] \equiv \mathrm{ED} \perp \mathrm{PD}$ on which is exerted also the Universal Quantized force , Gravity . This heavier Gravity -Force in $\mathrm{DM}$ is equal to $\mathrm{FD}=|\overline{\mathrm{q}}| \cdot[\mathrm{ED}+\overline{\mathrm{v}} \cdot \mathrm{PD}]$ and is exerted on any movable particle with charge $\overline{\mathrm{q}}$ as in gravity field is Since for three Units is applicable the same and in Material Geometry then, 
$\rightarrow\left[\left|\mathrm{c}(-\overline{\mathrm{W}} \cdot \mathrm{r})^{2}\right| \leftrightarrow\left|+\mathrm{c}(\overline{\mathrm{W}} \cdot \mathrm{r})^{2}\right| \leftrightarrow\left|-\mathrm{c}(\overline{\mathrm{W}} \cdot \mathrm{r})^{2}\right|\right]=$

c $\left[\left|(-\overline{\mathrm{W}} \cdot \mathrm{r})^{2}\right| \leftrightarrow\left|+(\overline{\mathrm{W}} \cdot \mathrm{r})^{2}\right| \leftrightarrow\left|-(\overline{\mathrm{W}} \cdot \mathrm{r})^{2}\right|\right]=\mathrm{c} .\{\leftarrow 0 \rightarrow\}$

i.e. Gravity travelling at light speed $=\mathrm{c}|\Lambda| \equiv \mathrm{c}$ Spin

So, Gravity force is the minimum attractive and biding Force $\square \mathrm{i}=2(\mathrm{wr})^{2}$ on dispersion $[\mathrm{MFMF}] \rightarrow\left[\left|+(\overline{\mathrm{w}} \cdot \mathrm{r})^{2}\right| \leftrightarrow \mid\right.$ $\left.-(\overline{\mathrm{W}} \cdot \mathrm{r})^{2}|=| \lambda \mid\right] \leftarrow$ and is the material energy length consisted of material points $\left|(\mathrm{wr})^{2}\right|$ which are the two $\pm\left|(\mathrm{wr})^{2}\right|$ dipole and restrained by the above Electromagnetic field E,P becoming from the binding force $\mid 2$ (wr) ${ }^{2} \mid$ and having the equations,

$\mathrm{E}=2 \mathrm{~A} \cdot \operatorname{sinkx} \cdot \cos w t=\sqrt{2} \cdot\left|(\mathrm{wr})^{2}\right| \cdot \operatorname{sinkx} \cdot \cos w t$,

$P=2 A \cdot \sin k x \cdot \cos w t=\sqrt{2} \cdot\left|(w r)^{2}\right| \cdot \operatorname{sinkx} \cdot \cos w t$,

in our Planck`s confinement cave, and which interact with all other particles . Since acceleration ,

$\mathrm{a}=\mathrm{d} \overline{\mathrm{v}} / \mathrm{dt}=(\mathrm{d} N / \mathrm{dt}) . \mathrm{f}$, is analogues to wavelength,$\lambda$, and to frequency, $\mathrm{f}$, then for,$\lambda=$ any constant $($ or zero $)$, then $\overline{\mathrm{v}} \rightarrow 0$ and $\mathrm{f} \rightarrow \infty$.

This is the why the very strong gravitational fields are present and close to black holes where there $\overline{\mathrm{v}}=$ $\overline{\mathrm{c}}=0$ or any constant. [40-41], For more in [51].

The motion of this DM,DE, mixture of the spherical opposite signed materials and dipole energy sector volumes , is not in contrary to gravity force, because both have already passed from the center of STPL contracted mechanism. Gravity field is the Rest base of all universe which doesn`t exist apriori but continually created as above and is the Base, the carpet, on which the Dark-energy Dark-matter heap mixture, with light velocity , $\bar{c}$, is rolling, expanding, with the maximum constant ,c, and continually formulating the ,

\section{Zero $\rightarrow$ Discrete $\rightarrow$ Infinite Geometrical Universe.}

symbolism

In $[22-23] \leftrightarrow$ Monad $\mathrm{AB}$ is the dipole,$(\mathrm{PA} \leftrightarrow \mathrm{PB})$, or $[\{\mathrm{A}(\mathrm{PA}) \leftarrow 0 \rightarrow(\mathrm{PB}) \mathrm{B}\}]$ and it is the

of the two opposite forces (PA), (PB) at points A,B which vibrate perpendicularly as E-M wave in energymonad $|\mathrm{AB}|$, and are created as force at the edge points $\mathrm{A}, \mathrm{B}$.

This Symbolism of primary point ( i.e. the zero , 0 , which is nothing ) shows the how creation of Opposites, A and $\mathrm{B}$, points becomes from this zero point A, which is Non-existence to Existence B , in Space monad AB , i.e. the quantization of point $A$ to point $B$ as the dipole $A B$ is as , the $[S] \leftrightarrow[A S]=[$ Space $] \leftrightarrow[$ Anti-space] mechanism.[12]

Above dipole Space-Anti-Space quantization of the Six-Triple-Points-Line Mechanism, produces and transfers Points of Spaces , Anti-Spaces and Sub-Spaces in a Common Inertial Sub-Space and a cylinder, in Gravity field [MFMF] and Particles , and describes the Space -Energy beyond Plank's length level [Gravity Length 3,969.10-62 m ], reaching the Point $=\mathrm{Lv}=$ ei. $(\mathrm{Nm} 2) \mathrm{b}=10 \mathrm{~N}=-\infty \mathrm{m}=0 \mathrm{~m}$, which is nothing and zero space.[43-46]. The very clear conclusion is that ,

Breakages located IN Cylinder $\rightarrow$ Acquire

Oscillation from their inherent Vibration derived from velocity vector $\bar{v}=>\bar{c}$, while

Breakages located OUT of Cylinder $\rightarrow$ Acquire

Oscillation from their interrelation, bonding .

Forces, Pressure, Velocity, Work (Energy-Space), Equivalence .

\section{A.. Equations of Elasticity :}

Principle stresses $\sigma 1=\sigma / 2[1+\sqrt{5}], \sigma 2=\sigma / 2[1-\sqrt{5}]$,

where exists $\sigma 1 . \sigma 2=\sigma^{2}$ on a surface, and it is

$\sigma 1=\mathrm{E} . \varepsilon(\mathrm{u})=$ The inward compressible pressure (in radial displacement distance).

$\sigma 2=\mathrm{G} . \varepsilon(\mathrm{v})=$ The transverse stretchable pressure ( the perpendicular to radial displacement distance).

$\mathrm{E}=$ Young`s modulus (linear stress/strain)

$\mathrm{G}=$ Shear modulus (transverse stress/strain) and holds $\mathrm{E} \perp \mathrm{G}$

B.. Equations of motion -Viscously Damped Free Vibration -Homogenous equation $m \ddot{x}+c \dot{x}+k x=0$ and is composed of $\mathrm{x}=\mathrm{A} \cdot \sin \cdot \mathrm{wt}, \dot{\mathrm{x}}=\mathrm{wA} \cdot \sin (\mathrm{w} t+\pi / 2), \ddot{\mathrm{x}}=\mathrm{w}^{2} \mathrm{~A} \cdot \sin (\mathrm{w} t+\pi)$, where $\mathrm{m}=$ mass $=$ is a Reaction to the distance coefficient proportional to velocity changes and is directed to velocity vector $\bar{v} r$,

$\mathrm{c}=$ The Damping coefficient directional to velocity vector $\bar{v} \mathrm{q}$,

$\mathrm{k}=$ The stiffness coefficient directed to velocity vector $\overline{\mathrm{v}} \mathrm{r}$, and holds between coefficients , $\mathrm{m} / / \mathrm{k} \perp \mathrm{c}$

C.. Equations of Maxwell`s Displacement Current : Electric Displacement field $\rightarrow \mathrm{D}=\varepsilon$.E $+\mathrm{P}$, Magnetic field

$\rightarrow \mathrm{B}=\mu . \mathrm{H}$ where ,

$\varepsilon=$ Permittivity $=$ Dielectric constant multiplier, i.e. a measure opposing displacement distance between electric field $\mathrm{E}$, and electric flux density $\mathrm{D}$.

$\mu=$ Dielectric constant multiplier, i.e. a measure opposing the perpendicular to displacement rotation between the inner vortex density $\mathrm{H}$ and the external weighted vorticity density $\mathrm{B}$. i.e.

Dielectric constant multiplier $\varepsilon \perp \mu$. 


\section{Remark:}

Work as Energy is Quantized, converted, in Space monads, caves ,cells, $\overline{\mathbf{x}}=\mathbf{d} \overline{\mathbf{s}}=\mathbf{\lambda} \mathbf{m}$, as pressure $\sigma$ , $\mathrm{T}$, the pressure is converted in caves as a Standing Electromagnetic Wave $\mathbf{E} \perp \mathbf{P}$ which consists the Standing monad ( Displacement current ) and the moving Energy monad ( by altering the inner wavelength or Period of monad ) in Gravity`s field medium [MFMF] and is dissipated as Quaternion monads ( Particles or Waves , matter or vectors) as Forces ( displacements, masses, pressure etc .) using modulus , coefficients , reactions to the motion and all other geometrical indices.

Stability In-Out wavelength is obtained by the Isochronous Anti-Standing Electromagnetic Wave $\mathbf{E} \perp \mathbf{P}$ which happens on Anti-cycloid, Evolute, and whirling, Curl, by the equality of the two transverse Complex Envelope displacements, Amplitudes, that consist the Quantized Standing Energy monad (and Outer as Coriolis force, Spin Helix , the Displacement current in monads) .

7.1. The Energy of Particles and Spin .

Conservation of energy in an, free vibration un-damped system, becomes partly as kinetic ,T, (stored in the mass by virtue of its velocity and for mass-less in velocity vector) and partly potential $U$ (stored in the form of strain energy in Elastic deformation of work done in a force field ) .

In case of material point i.e. a $\operatorname{monad} \overline{\mathrm{z}}=\mathrm{s}+\overline{\mathrm{v}} \mathrm{i}=$

$\mathrm{s}+\overline{\mathrm{v}} \cdot \nabla \mathrm{i}$, that what is called mass, coincides with the Intrinsic Electromagnetic field $\mathrm{E} \perp \mathrm{P}$ of the two transverse waves E,P in monad as before, the mass

$\mathbf{m}_{\mathbf{p}}=\frac{32}{3}\left(\pi \mathrm{r}^{3}\right) \cdot\left[\varepsilon \mathrm{E}^{2}+\mu \mathrm{H}^{2}\right]$ where ,

The two transverse waves consist the Energy quanta in cave $\rightarrow \quad\left[\left|+(\overline{\mathrm{W}} \cdot \mathrm{r})^{2}\right| \leftrightarrow\left|-(\overline{\mathrm{W}} \cdot \mathrm{r})^{2}\right|=|\lambda|\right]=4 . \pi \cdot \mathrm{r} \leftarrow$ and Volume $=323\left(\pi^{2} \mathrm{r}^{3}\right)$ of rolling circle radius $\mathrm{r}$.

Because the cross product of two equal vectors , $\overline{\mathrm{v}}$, is vector $|\bar{v}|^{2}=\overline{\mathrm{v}} \mathrm{x} \overline{\mathrm{v}}$, perpendicular to area of the two vectors, with real part equal to inner product area $\quad|\overline{\mathrm{v}}| \mathrm{x}|\mathrm{v}|=|\mathrm{v}|^{2}$, perpendicular vectors $\overline{\mathrm{v}}$, formulate the two transverse waves on cycloid which produce the Angular Momentum of monad and the equilibrium Anti-Angular Momentum on Evolute. This Intrinsic Angular Momentum consists Work in monad and is called Spin and it is the property of all elements as ,

1). $\left[ \pm \overline{\mathrm{v}} . \mathrm{s}^{2}\right] \rightarrow$ Fermions and $\rightarrow[\overline{\mathrm{v}} . \nabla \mathrm{i}] \rightarrow$ Bosons,

2). $\left[ \pm \mathrm{s}^{2}\right] \rightarrow[\mathrm{MFMF}]$ Field, and the binder, Field

$[\nabla \mathrm{i}] \rightarrow$ Gravity force

3). $\left[ \pm \bar{c} \cdot s^{2}\right] \rightarrow$ Dark matter, and the binder Gravity

force $[\nabla \mathrm{i}],[\overline{\mathrm{c}} . \nabla \mathrm{i}] \rightarrow$ The Expanding Dark

energy . [39,40,42].

Energy Quantities $\left.\nabla \mathrm{i}=2(\mathrm{wr})^{2}\right]$ acting, on dipole breakages $\left[ \pm \overline{\mathrm{c}} \cdot \mathrm{s}^{2}\right]$ or on

$\mathrm{DM}$ field $=[\mathrm{Em}+\overline{\mathrm{c}} \cdot \mathrm{Pm}]=\left[\mathrm{c} \cdot \mathrm{Eg}+\overline{\mathrm{c}}^{2} \cdot \mathrm{Pg}\right] \rightarrow\left[\left|+\mathrm{c} \cdot(\overline{\mathrm{w}} \cdot \mathrm{r})^{2}\right| \leftrightarrow\left|-\mathrm{c} \cdot(\overline{\mathrm{w}} \cdot \mathrm{r})^{2}\right|=|\lambda|\right] \leftarrow$, produces DM, while Dark-energy $\mathrm{DE}=[\overline{\mathrm{c}} . \square \mathrm{i}]$ is acting on all Five Fragments with light velocity,$\overline{\mathrm{c}}$, as,

$[\bar{c} . \nabla \mathrm{i}] \rightarrow\left\{(\nabla \mathrm{i}),\left(+\mathrm{s}^{2}\right),\left(-\mathrm{s}^{2}\right),\left(+\mathrm{cs}^{2}\right),\left(-\mathrm{cs}^{2}\right)\right\} \leftarrow$

which is the rolling Heap. [33-36]. All the Energy Quantities $\left.\square \mathrm{i}=2(\mathrm{wr})^{2}\right]$ in the rolling Heap, acting on dipole breakages $\left[ \pm \mathrm{s}^{2}\right]$ formulate Gravity-Field and Gravity-force while acting on dipole breakages

$\left[ \pm \bar{c} . s^{2}\right]$ formulate Dark matter and Dark Energy respectively, while acting on Leptons and Quarks , AntiLeptons and Anti-Quarks, Bosons formulate the Material worlds. A wide analysis of all in [48].

The Work Quantization process is as below,

Energy and Space or both as follows , [ F.2 ],

1..Work W $=$ F.ds $-[\mathrm{ds}=0, \mathrm{t}=0, \mathrm{~F} \neq 0] \rightarrow$ Stationary Force on zero Displacement $\rightarrow$ and is transformed as, The Material Geometry and the Origin of Energy-Particles in Periodic System 2..Work $\mathrm{W}=(\sigma . \mathrm{dF}) . \mathrm{ds}-[\mathrm{ds}=0, \mathrm{t}=0$, $\mathrm{dF} \neq 0] \rightarrow$ Stresses on any Area, and if area is zero then is transformed as,

3..Work $\mathrm{W}=(\mathrm{c}[\varepsilon \mu]) . \mathrm{ds}-[\mathrm{dF}=0, \mathrm{ds}=0, \mathrm{t}=0] \rightarrow$ Permittivity-Permeability of Electromagnetic field and is transformed as,

4.. Work $\mathrm{W}=(\mathrm{E} \square \mathrm{H}) \cdot \mathrm{ds}-[\mathrm{dF}=0, \mathrm{ds}=0, \mathrm{t}=0] \rightarrow$ Inner Electromagnetic Wave of monad is transformed as ,

5.. Work $\mathrm{W}=(\lambda . \mathrm{m}) \cdot \mathrm{ds}-[\mathrm{dF}=0, \mathrm{ds} \neq 0, \mathrm{t}=0] \rightarrow$ Moving Field Vector and for $\mathrm{dF}=0, \mathrm{ds} \neq 0$ is transformed as,

6. . Work $\mathrm{W}=(\mathrm{p}) \cdot \mathrm{ds}-[\mathrm{dF}=0, \mathrm{ds} \neq 0, \mathrm{t}=0] \rightarrow$ Angular Momentum of the moving field, and is transformed as, Space, and their combination as .

7.. Work $\mathrm{W}=(\mathrm{h} / \mathrm{v}) . \mathrm{ds}-[\mathrm{dF}=0, \mathrm{ds} \neq 0, \mathrm{t}=0] \rightarrow$ Cycloidal Spin, in wavelength,$\lambda$,

Work as Energy $=\mathbf{h . f}$ which is the known

Plank`s formula for frequency and energy.

$\mathrm{W}=(\sigma \cdot d \mathrm{~F}) \cdot \mathrm{ds}=\sigma \cdot(\mathrm{v} \cdot \mathrm{v})=\sigma \cdot\left(\mathrm{v}^{2}\right)=(\lambda \mathrm{m}) .\left(\mathrm{v}^{2}\right)$

$\left[\right.$ Mass $\left.\cdot \mathrm{v}^{2}\right]=\mathbf{m} \cdot \mathbf{c}^{2}-[\mathrm{dF}=0, \mathrm{ds} \neq 0, \mathrm{t}=\mathrm{t}] \rightarrow$

Work as Kinematic Vector which is the known

Einstein`s formula for mass and energy and is transformed as, 
8..Work $\mathrm{W}=(\lambda \cdot \mathrm{m}) .(\mathrm{v} . \mathrm{t})-[\mathrm{dF}=0, \mathrm{ds} \neq 0, \mathrm{t}=\mathrm{t}] \rightarrow$ Kinematic Vector with mass and velocity, and is transformed as,

9.. Work $\mathrm{W}=(\mathrm{M})$.v.dt $-[\mathrm{dF}=0, \mathrm{ds} \neq 0, \mathrm{t}=\mathrm{t}] \rightarrow$ General mass-Velocity moving vectors , and is transformed as ,

10.. Work $\mathrm{W}=(\mathrm{p}) . \mathrm{dt}-[\mathrm{dF}=0, \mathrm{ds} \neq 0, \mathrm{t}=\mathrm{t}] \rightarrow$

Linear Momentum as Extrema for Angular . The Work Quantization process $\rightarrow$ i.e. Work

Energy and Space or both ( Not Evolution )

is as follows , [12, $40-41]$. In Figure.9,

The three processes of quantization become from the two Substances of Energy-Space universe, Energy and Space, and their combination is as

a.. The Work Quantization process as Energy is

$\mathrm{W}=\mathrm{P} \cdot \mathrm{ds}=(\sigma \cdot \mathrm{dF}) \cdot \mathrm{ds}=(\sigma \cdot \mathrm{v} \cdot \mathrm{dF}) \cdot \mathrm{dt}=(\mathrm{p}) \cdot \mathrm{dt}$

b.. The Work Quantization process as Space is

$\mathrm{W}=(\mathrm{c}[\varepsilon \mu]) \cdot \mathrm{ds}=(\mathrm{E} \perp \mathrm{H}) \cdot \mathrm{ds}=$

$=[\mathrm{dt} .(\mathrm{E} \perp \mathrm{H})] \cdot \mathrm{ds} / \mathrm{dt}=\mathrm{m} . \mathrm{v}$

c.. The Work Quantization process as Energy

and Space is $\mathrm{W}=(\lambda \cdot \mathrm{m}) \cdot \mathrm{ds}=(\mathrm{h} / \mathrm{v}) \cdot \mathrm{ds}=$

$(\lambda \cdot \mathrm{m}) .(\mathrm{v} . \mathrm{t})=(\mathrm{M}) \cdot \mathrm{v} \cdot \mathrm{dt}$

\subsection{Work as Impetus, Momentum and Energy}

produced in all Systems, is Conserved.

Empty Space is Isotropic when all its Directions are Equivalent i.e., all Relatively Natural Sizes and Laws remain Inalterable with Rotation, therefore is in effect via , master-meters .[45-47]

Any two Energy-Systems (a) and (b), where, R (a) = The Place (Position) of the System (a), U (a) = The total Impetus of System (a). La = The Impulse of any System from a fixed

beginning, reference, point 0 , is holding, Total Impulse $L=L a+L b=R(a) \cdot F(a)+R(b) \cdot F(b) R(a) \cdot U(a)+R$ (b).U(b) = Constant .

The Dynamic Energy of System (a), (b) don't depend on Time, but only from the Relative Places of the Elements where are the points, Master -Meters, i.e. exists Energy-Space and not Space-time .

Work $\mathrm{W}=$ Fab. $\square \mathbf{a}=$ ma. $(\mathrm{dUa} / \mathrm{dt}) \cdot \mathrm{dr}=1 / 2 \mathrm{~d}\left[\mathrm{ma}_{\mathrm{U}} \mathrm{Ua}^{2}\right]+$ Fba. $\mathrm{rb}=1 / 2 . \mathrm{d}\left[\mathrm{mb} \cdot \mathrm{Ub}^{2}\right]$, therefore, Dynamic Energy change is $\delta \mathrm{V}=-$ Fab.ora-Fba.drb , and $\rightarrow \mathrm{D}-\mathrm{W}=\mathrm{dV}$, and as $\mathrm{T}$, is kinetic Energy, $\mathrm{dV}=\mathrm{d}\left[1 / 2 \cdot \mathrm{ma}^{\mathrm{U}} \mathrm{Ua}^{2}+\right.$ 1/2.mb.Ub ${ }^{2}$ ] or $\mathrm{d}(\mathrm{V}+\mathrm{T})=0$ and, $\mathbf{V}+\mathbf{T}=$ Constant.

Above conclusion define the, time moments, or Time periods, to be a relative measure of changes in any Dynamic Energy system and not essence, Continuous or Discrete. The called Empty space and generally the beyond gravity space is,$\rightarrow$ The Energy -Space -Continuum $\leftarrow$ and not Space-time as in GR referred . i.e.

Work, as Dynamic , V, and Kinetic , $\mathbf{T}$, energy is Constant and is Conserved, Quantized , as in [39] The Work $\rightarrow$ as Impetus , Angular-Momentum and Energy $\leftarrow$ produced in all Systems, is Conserved , ( Quantized ), But How ??

Since Angular-momentum is Work and is conserved, therefore Material Points, which such are Monads and Particles are unaffected when rotating .

It was shown that Particles have intrinsic Spin which becomes External and produces their motion. As above and else proved, this Energy-magnitude is conserved. The How is because, since this Property of Spinning-axis of rotation becomes from a Wheel-Rim which is free to assume any Orientation ,it exists on Gyroscope-Mechanism only, where on it the three RIM-Frames, with one and two degrees of rotational freedom are maintaining and tilting Spinning-axis, and because Physics become from Space, and Space becomes from E-Geometry only.

It was also proved for mass that, Mass ,m, is the magnitude, Meter, the Index , of changes either , those of Velocity, or that of Displacement or both, and or the same also $\rightarrow$ Force is equal to the change of Momentum and consists the conservation law of Work in this Energy-Space , Cosmos . markos 1/5/2016 


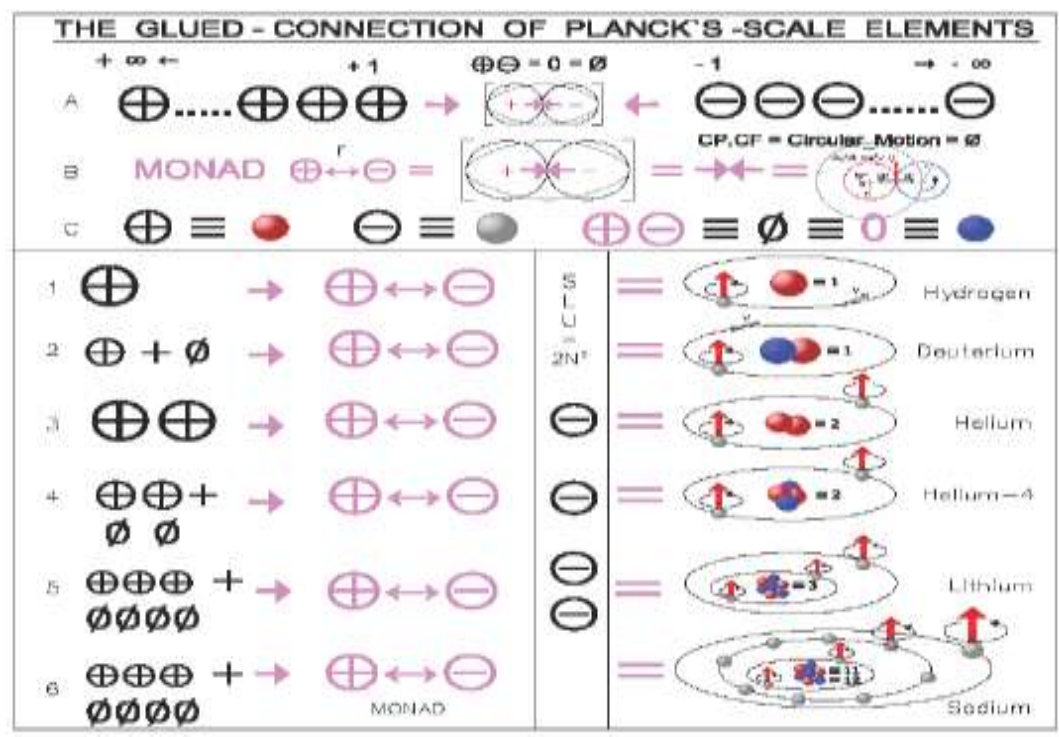

Figure. 7.

The Positive $\oplus$, Negative $\Theta$, and Neutral $\varnothing=\ominus \bigoplus$ with $\mathrm{r}=0$, Glued connection. Above Glued-connection of Under Planck`s Scale Breakages , creates the NEW $\oplus, \ominus, \varnothing$

\section{Material-Particles which form Atoms of the Known Periodic Table .}

A. The Series of Positive Breakages $\bigoplus$ is the Nucleus Cluster Structure, Zero $=$ Neutral $=\bigoplus \ominus$ is the Rest Monad Dipole, The series of Negative breakages $\Theta$ is the Orbital Cluster Structure.

B. The Monad of Positive $\oplus$ and Negative $\Theta$ breakages with $r=$ Distance, which is thus resulting to the Circular - Motion, because of the CentriPedal , CP, and of CentriFugal , CF, equal Forces .

C. The Positive $\bigoplus$ and Negative $\Theta$ Breakages, and the Zero $=$ Neutral $=\varnothing=\bigoplus \Theta$ with $r=0$. The Material Geometry and the Origin of Energy-Particles in Periodic System 1.. One Positive $\oplus$ Breakages, Attached to Nucleus of Monad $[\Theta \leftrightarrow \bigoplus]$ is Produced H11 = Hydrogen.

2.. One Positive $\bigoplus$ Breakages and one Neutral , Attached to Nucleus of Monad $[\Theta \leftrightarrow \bigoplus]$ is Produced H12 = Deuterium which is Isotopes of Hydrogen .

3. Two Positive $\bigoplus$ Breakages, Attached to Nucleus of Monad $[\Theta \leftrightarrow \oplus]$ is Produced He21 = Helium .

4.. Two Positive $\bigoplus$ Breakages and Two Neutral, Attached to Nucleus of Monad $[\Theta \leftrightarrow \bigoplus]$ is Produced He24 = Helium - 4 which is Isotopes of Helium .

5.. Three Positive $\bigoplus$ Breakages and Four Neutral, Attached to Nucleus of Monad $[\Theta \leftrightarrow \oplus]$ and using SLU= Shell-Least-Units Principle to Orbital Cluster , is Produced Li37 = Lithium .

6.. Eleven Positive $\oplus$ Breakages and Twelve Neutral , Attached to Nucleus of Monad [ $\ominus \leftrightarrow \bigoplus]$ and by using SLU = Shell-Least-Units Principle to Orbital Cluster , is Produced Na1123 = Sodium, and so on

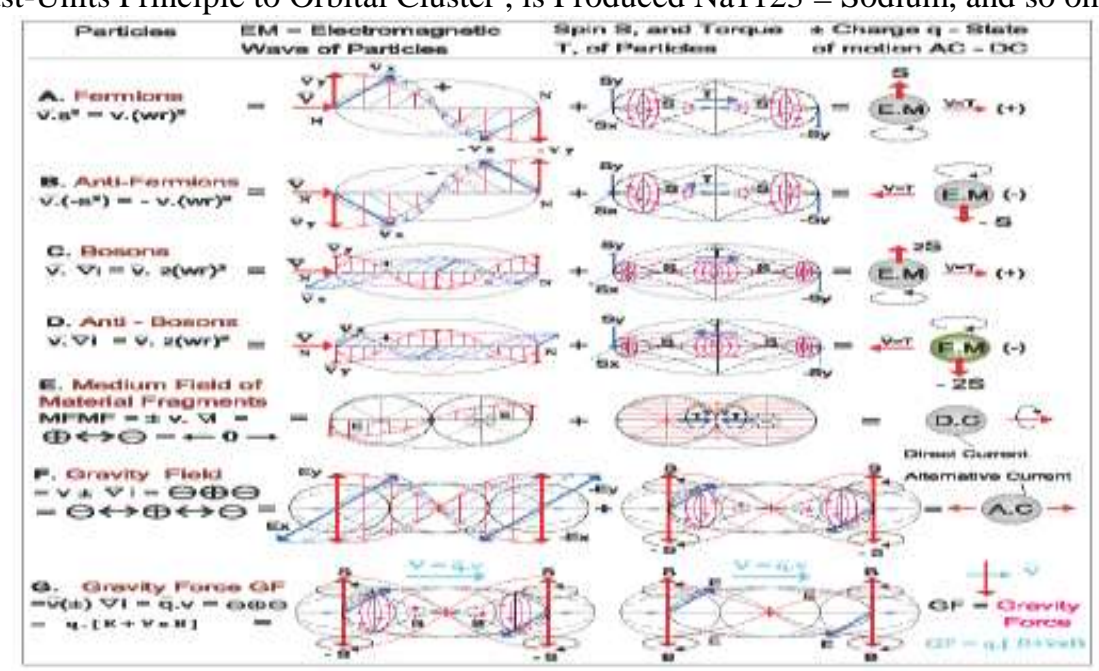

Figure. 8. 
The Collision of Light-velocity Vector on Material Breakages creates, the Particles and the Rest Forces on Cycloidal - Evolute motion .

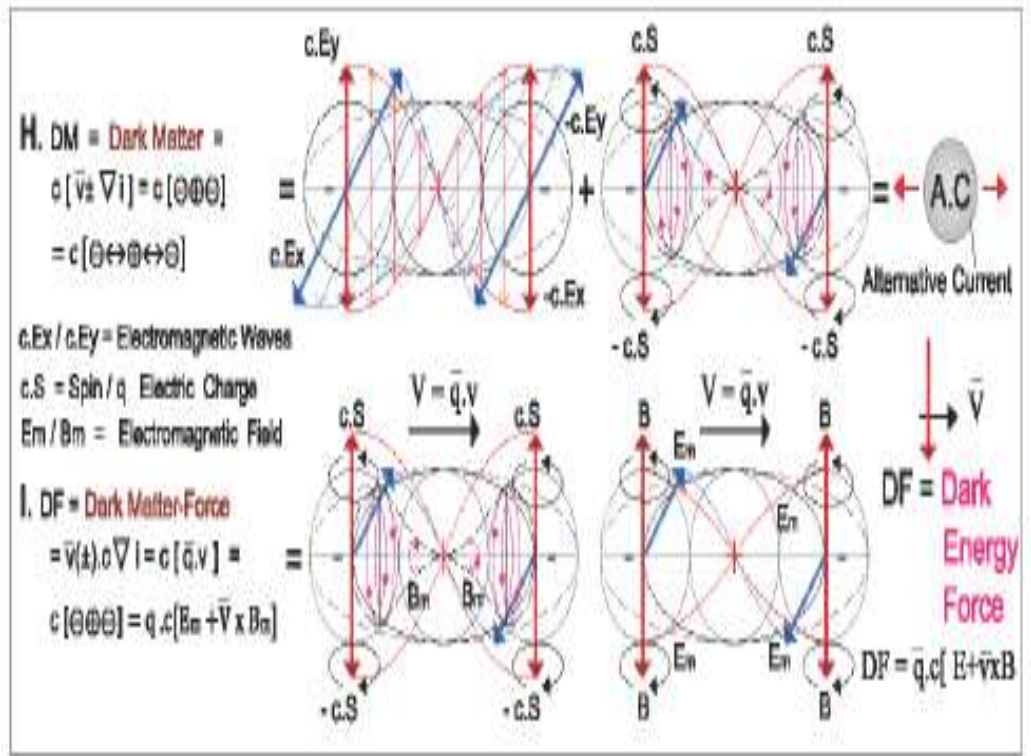

Figure. 9.

The Collision of Light-velocity Vector on Material Breakages creates, the Rolling Dark-Matter and the Dark-Matter Forces on Cycloidal - Evolute motion .

The Inverse Circle-Cycloidal , on the $\bigoplus$ Relief , of Well $\ominus$ Rotation :

Since Material Geometry follows Euclidean therefore series of Positive Numbers added to any Number consist the Sum of Positive Numbers, while series of Negative Numbers added to any Number consist the Sum of Negative Numbers and the whole consists the Infinite Number series. The two series are jointed to Zero where Zero is equal to the Sum of Positive and Negative series.

In Figure 7, 10 , Interactions of opposite are the Centripetal Force, $\mathrm{CP}$, which is the Action to the equal and opposite reaction Centrifugal Force, $\mathrm{CF}$, according to Newton`s third law .

This Centripetal force $\bigoplus$ is required so that the Negative breakage $\ominus$ by Rolling on Positive breakage circumference, to experience Uniform Circular motion, towards their axis, and to maintain its circular path , i.e. Centrifugal and Centripetal Forces exist .. This Geometric logic is just the opposite to the circular motion becoming from the two above referred forces.

The Well is Zero and all the Negative series of Numbers $(-\infty$.. -N , 0$)$ are into Zero , i.e. The Space, $\bigoplus$, Positive numbers, the Anti-Space, $\ominus$, Negative numbers are in Sub-space, Zero $=\ominus$, which is the Neutral space . The Material Geometry and the Origin of Energy-Particles in Periodic System

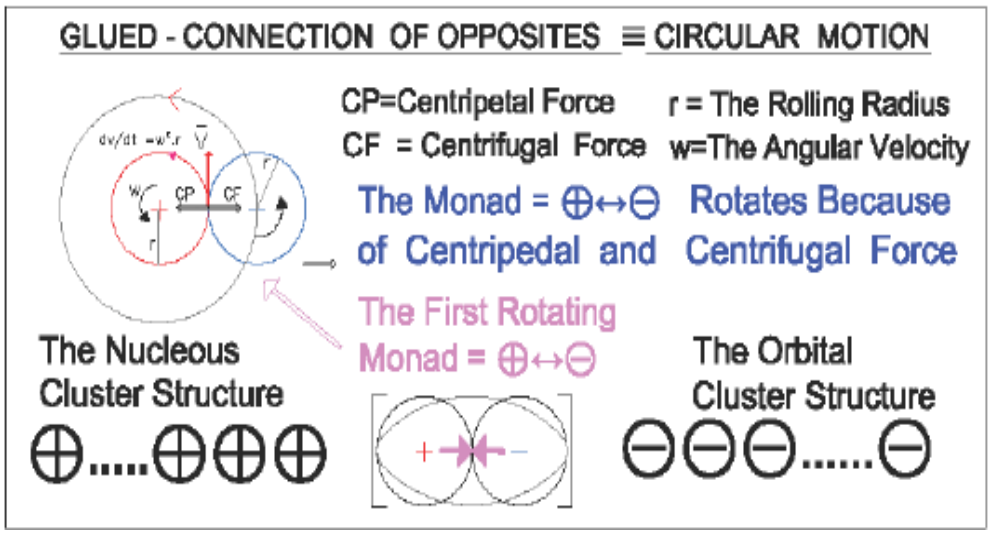

Figure 10.

The Uniform Circular motion is the First Possible Position of all Glued-breakages for the Under Planck`s Scale Monads due to the Glued-Bond of Centripetal and Centrifugal Forces. 
1. ${ }_{1}^{1} \mathrm{P} . . \ominus \mid \oplus=\ominus 0 \oplus==$ The Set of Negative-Numbers, Point $\rightarrow O$, The Positive - Numbers, with $\mathrm{ds}=0$ While $[\oplus \leftrightarrow \ominus]=$ Material Point of $d s=r=$ Monad = The two Inverse Perpendicular Units, creating Zero Work and this because , $\{$ Interaction $\leftrightarrow$ in Bonding is, $\bar{v} \perp \bar{r}$, to the Centripetal Force \}, and for Planck`s

2. Length scale $10-35 \mathrm{~m}$, is The Hydrogen Bohr`s Model - Structure .

2. ${ }_{1}^{\mathrm{P}} \mathrm{P}$.. $\oplus \ldots . . \oplus \oplus \oplus[\oplus \leftrightarrow \Theta]=$ The Number of Positive Units, interacting in the Material Point, and for Planck`s scale 10-35 m , is The Nucleus Cluster - Structure of Positive Protons . Why ??

3. ${ }_{\mathrm{E}}^{1} \mathrm{P}$..[ $[\oplus \leftrightarrow \ominus] \ominus \Theta \Theta \ldots . . \ominus=$ The Number of Negative Units, interacting in Material Point, and for Planck`s scale 10-35 m , is The Orbital Cluster - Structure of Negative Electrons . Why ??

4. ${ }_{\mathrm{E}}^{\mathrm{P}} \mathrm{P} . . \oplus \ldots . . . \oplus \oplus \oplus[\oplus \leftrightarrow \ominus] \ominus \ominus \ominus \ldots . . \Theta=$ The Number of Positive and Negative Units interacting on The Zero Material point $\{\overline{\mathrm{v}} \perp \overline{\mathrm{r}}\}$, is The Proton and Electron on Material Point , and for Planck`s scale $10-35$ m, The Periodic -Table of Elements.

The combination of $\rightarrow$

The Nucleus Cluster - Structure of Positive Protons,

The Orbital Cluster - Structure of Negative Electrons, and The SLU = Shell Least Units , creates the Periodic Table of Elements, and this because exist only the thrust vectors $\nabla \mathrm{i} \equiv \oplus$ creating Electromagnetic Waves and External Spin.

Following above logic, it is easy on 4.. to define particles beginning from Zero which is Point $\rightarrow \mathrm{O}$ to $\mathrm{P}=1, \mathrm{E}=1$ which is Hydrogen, to $\mathrm{P}=2, \mathrm{E}=2$ which is Helium, to $\mathrm{P}=3, \mathrm{E}=3$ which is Lithium, to $\mathrm{P}=4, \mathrm{E}$ $=4$ which is Beryllium, to $\mathrm{P}=5, \mathrm{E}=5$ which is Boron, and so on for all Particles of the Periodic Table as is shown in Fig.7 . For the Full Orbital Electrons are, $\mathrm{P}=1, \mathrm{E}=1$ for Hydrogen , $\mathrm{P}=2, \mathrm{E}=2$ for Helium , $\mathrm{P}=10$, $\mathrm{E}=10$ for Neon $, \mathrm{P}=18, \mathrm{E}=18$ for Argon $, \mathrm{P}=30, \mathrm{E}=30$ for Krypton, $\mathrm{P}=54, \mathrm{E}=54$ for Xenon, $\mathrm{P}=86 \mathrm{E}=86$ for Radonion , $\mathrm{P}=118, \mathrm{E}=118$ for Onunoctium .

\section{Analysis follows .}

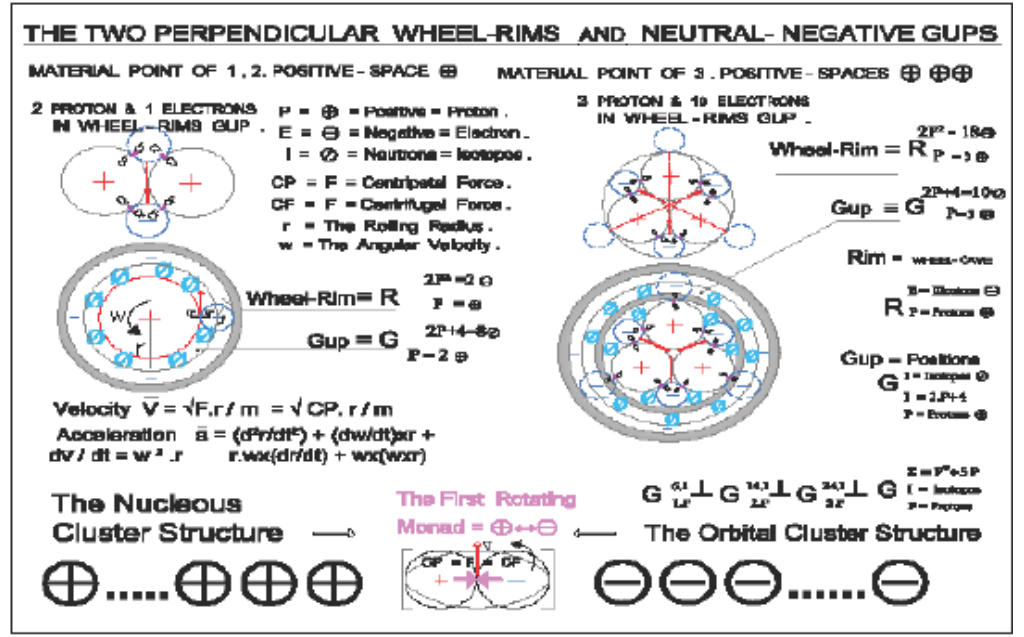

Figure 11.

Material-Geometry of the Nucleus, $\bigoplus$, are the Rotating - Monads $[\Theta \leftrightarrow \oplus]$, of the Wheel-Rims which are Perpendicular to the Rotor`s plane, consisted of the same Rotating Monads $[\oplus \leftrightarrow \ominus]$.

7.3. The First Rotating Monad .

1.. Material point is defined as Positive, $\mathrm{s}^{2} \equiv \bigoplus$, and Negative, $-\mathrm{s}^{2} \equiv \ominus$, Particles becoming from STPL Mechanism . [18-19]

2.. The two Particles, when in contact $\bigoplus \ominus$, equilibrium because of the two opposite and equal action forces $F$ , - F, on their common point . Because the two Forces, the Glued-Bond, are opposite and continually acting on this common point then according to Newton`s third law, This Common Point executes a circular motion with velocity $\mathrm{v}==\sqrt{F \cdot \frac{r}{m}}$ where, $\mathrm{r}=$ the radius of the $\oplus$ breakage and $\mathrm{m}=$ the mass of the $\Theta$ breakage $=$ The reaction to the change of Velocity direction .

Acceleration $\overline{\mathrm{a}}=\left(\mathrm{d}^{2} \mathrm{r} / \mathrm{dt}^{2}\right)+(\mathrm{dw} / \mathrm{dt}) \mathrm{xr}+\mathrm{rw} \mathrm{x}(\mathrm{dr} / \mathrm{dt})+\mathrm{w} \mathrm{x}(\mathrm{wxr})$ in vector form .

3. The conclusion from above is that, The continuous Contact of opposite creates Circular Motion, i.e. An Energy Wheel-Rim or a Cave in where Negative breakage, the Electron, of radius $r$ and mass $m$, Cycloidally Rotate on Positive breakage, the Nucleus, of the same radius r. Because of the Cycloidal motion, the center of mass is in the center of Negative breakage and is rolling in Circular motion so Velocity vectors define One Gap 
on both sides, the One between Inner Velocity Vector and circumference of the Positive which is called ,The Neutrons Gap, and the Second between the Inner Velocity Vector and the Wheel-Rim, which is called, The Orbit or Orbital.

The Positive breakage is called the Nucleus of this System-monad .

4.. The Gap of the Energy Wheel-Rim is dependent on the Positive $\bigoplus$ breakage and of the circular motion of angular velocity w, of the Negative $\Theta$ breakage, therefore can be filled with Negative $\Theta$ only , and this because, at the point of contact on the Rim, this Presure is always Positive .

The other Gap between the Velocity vector and the circumference of the Positive breakage cannot be filled with Negative breakages because already exist Rotation, therefore is filled with Neutrons, Isotopes .

The Obvious Inference is that the First-Gap can be filled with Neutrons Units only, While, The Second-Gap can be filled with Negative Units only, $\ominus$ i.e. Two different containers.

The How many Negative breakages, Units = Positions, can be filled, is dependent on the Possible Repetitive Permutations of Moulds, Positive $\bigoplus$ breakages, and Units which are 2 Units, it is the maximum number in a Point, i.e. The Possible Repetitive Permutations for Moulds and Units are 2.Mould ${ }^{\text {Units }}=2 . \mathrm{M}^{2}$, for every Mould $=$ Space $=$ Number of Non-coinciding Points , and from figure 12 the Available Extrema Positions Units for $\mathrm{M}=\mathrm{N}=4$, the Total Positions in Mould is $2.16=32$ Position-Units which are Electrons . For Neutrons Units is $2 \mathrm{~N}$ for N-Mould and for $\mathrm{N}=4$ is $2.4=8$ Position-Units.

5. The Positive $\bigoplus$ breakage can equilibrium with One Negative $\Theta$ breakage, when in conduct, $\oplus \Theta \oplus$, and since when $[\bigoplus \ominus]$ in conduct, is then created the 1rst Wheel-Ring . For the same Way and Why, Rotation of the 1rst Wheel-Ring on the 2nd Positive $\bigoplus$ breakage creates the Second Wheel-Ring in this closed system. i.e. , When Nucleus has two Positive $\bigoplus$ breakages $N=2$ and one Negative $\Theta$, then $\bigoplus \Theta$ Glued-contact of opposite creates the First Wheel-Rim R1E with E number of Negatives and Gap G1I with I number of Neutrons Units equal to $2 \mathrm{~N}=2(\mathrm{P}+2)$, which can be filled with $2 . \mathrm{N}^{2}=2$ Negative and $2.3=6$ Neutron Units only .

The $[\bigoplus \Theta] \oplus \equiv[\bigoplus \bigoplus] \Theta$ conduct of opposite creates the Second Rotating Wheel-Rim R2E with Gap G2I , in which second Wheel-Rim, Wheel-Rim R12 with Gap G16 are rotated with the same angular velocity w , and which Wheel-Rim also can be filled with $2 . \mathrm{N}^{2}=8$ Negative Units and 2(2+2) 8 Neutron Units only .

6.. Following the Prior logic of Spaces for , $\mathrm{N}=1 \rightarrow$ Material Point , $\mathrm{N}=2 \rightarrow$ The Line-Segment , $\mathrm{N}=3 \rightarrow$ The Plane, $\mathrm{N}=4 \rightarrow$ The Volume , $\mathrm{N}=5 \rightarrow$ The Regular Pentagon, $\mathrm{N}=6 \rightarrow$ The Regular Hexagon , N = $7 \rightarrow$ The Regular Heptagon, $\mathrm{N}=\mathrm{N} \rightarrow$ The Regular Plane or Space of equal angles N-gon, and since Positive $\bigoplus \equiv$ Protons $\equiv \mathrm{N}-2$, then in each Wheel-Rim there are N-2 Protons and 2N, Isotopes as Total number of them $\mathrm{P}$ and $\mathrm{P}^{2}+5 \mathrm{P}$ respectively, including all Prior Wheel-Rim and Gap number of Units . The Material Geometry and the Origin of Energy-Particles in Periodic System

Figure 12.

The Uniform Circular motion is the First Possible Position of Monads. The Number of Neutrons in Space represent Isotopes in Nucleus.

In 1. Euclidean Geometry is defined on the Number of Points which can define a Space, i.e.

The Point is defined from one Point, The Line Segment consisted of two Points, The Triangle consisted of three Points, The regular Tetragon consisted of four Points in, The regular Pentagon consisted of five Points in Space and so on, represent the Steady, stable, formations of Geometry.

In 2. Are shown the Material-Points, Positives and Negatives on each Point which is Zero and can be added to any other Positives and Negatives, and which represent Protons and Electrons.

In 3. Are shown the Number of Positive in Space and which represent Protons in Nucleus .

In 4. Are shown the Number of Neutrons in Space and which represent Isotopes in Nucleus .

In 5. Is referred the First Rotating Monad from Hydrogen-Shell to all Atoms .

In 6. Is referred the SLU = The maximum allowed Positions, Units, in each Wheel - Rim .

In 7. Are shown the different Filled Wheel-Rims with all prior Atoms in them . 


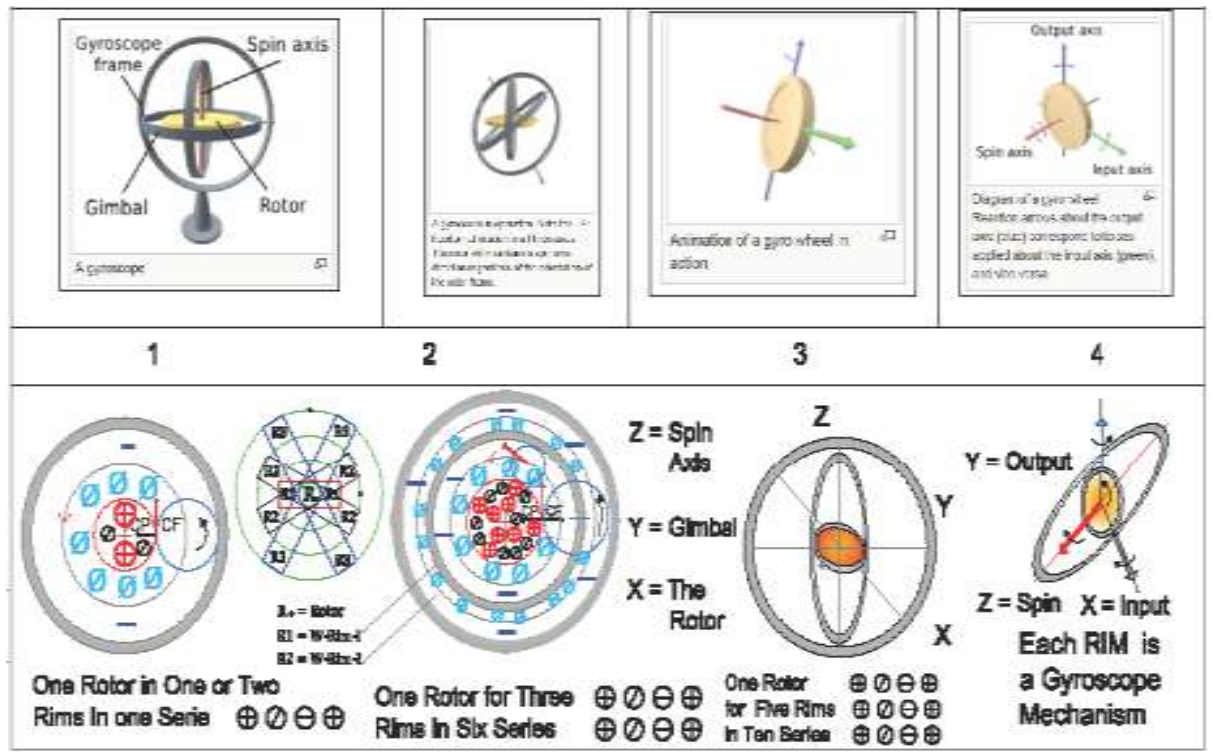

Figure 13.

The How Gyroscope is working and the Two Gyroscope-Stereo-Slice Mechanism -GSSM- Photos are from a Gyroscope where Reaction arrows about the Output axis, correspond to Forces applied about the Input . In 1.. Is shown the Rotor and Spin in one Rim . Velocity vector $\bar{v}$ separates the Space in two Containers , One for Protons by the Nucleus and One for Electrons by the Wheel-Rim .

In 2.. Are shown the Two Rotors and Spins-axis, the Two Rims where in each one exist two Gups, One for Protons by the Nucleus and One for Electrons by the Rim, and the Frame .

In 3.. Is shown the Rotor in $\mathrm{X}$ axis , the Gimbal in $\mathrm{Y}$ axis, and Spin in $\mathrm{Z}$ axis, all in the Gyroscope Frame and of different Stereo-Slices, shifted by ds $=2 \mathrm{r}$. This shifting in X, Y direction creates the Shape of the Pth Rim which is as the Human DNA. I say just the opposite, i.e. Human DNA and all other DNA of all monads become of the above referred shifting in order to have Positions, the Electrons and Neutrons .

In 4.. Animation of a Wheel-Rim is the Reaction arrows $\mathrm{Z}=$ Spin about the output axis $\mathrm{Y}$, which correspond to forces applied about the input axis $X$ and vice versa. Since each Resultance differs by ,ds, then the shape is a Double-helix as DNA. Because Spin-Resultance is conserved in Magnitude and direction , therefore -GSSMMechanism allows Monads, and the Light which is monad, to travel in X, Y direction and directed to Spin`s.

In Figure.15 . Each Wheel-Rim is a Gyroscopic-Mechanism which is successively placed on theStereo-Helix where Animation is orientated to X-Y-Z, and Spin - axis . The Material Geometry and the Origin of EnergyParticles in Periodic System

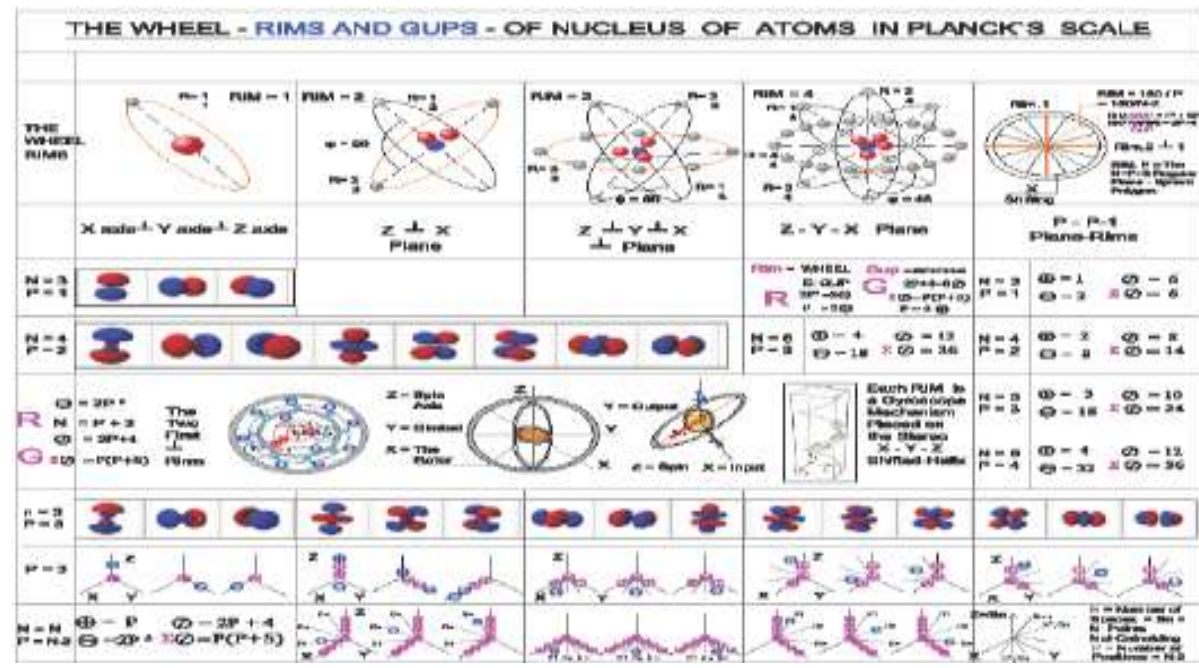

Figure 14.

The Uniform Circular motion is the First Possible motion due to the Position of Monads. In Row 1, are shown the Wheel-Rims, RP E with two Indices, the down one for each Positive $\bigoplus=$ Protons and the upper one 
for each Negative $\Theta=$ Electron Breakage. The number of Rims n-RIM = 180/P i.e. For $\mathrm{P}=1$ then Rim1 is a Flat rim with thickness $2 \mathrm{r}=2 \mathrm{~s}^{2}=2$ (w.r) ${ }^{2}$, obeying all Euclidean Plane rules. In this Rim exists one Proton and 6 Neutron Positions for the Rim-Isotopes, and 2 Electron positions.

For $\mathrm{P}=2$ then the second Rim is RIM $=180 / 2=90^{\circ}$ Slice-Stereo-angle i.e. perpendicular to R1 .

Rim2 is shifted to the Rim's slice in order to avoid simultaneous conflict and so accepts 8 Neutrons and 8 Electrons. This shifting defines the Helical shape of Rims. For P $=3$ then the third Rim`s RIM $=180 / 3=60^{\circ}$ Slice-Stereo-angle i.e. a Sphere with R3 Slice. All Rims are shifted between them to the Rim`s thickness for avoiding conflicting, and so accepts 18 Neutrons, and 10 Electrons and defining the Helical shape of Rims.

The three Rims are found in term in the next one, so all atoms differ each other in one Position only, either is Positive $=$ Proton, either is Negative $=$ Electron, either is Neutral $=$ Isotopes. Since Structure of Atoms follows Gyroscopic-Mechanism and E-Geometry Axioms, therefore Periodic-Table of Elements can be modelled in this logic, and since also was shown that Monads are Quaternions as Space-Energy, which move with their External Conserved-Spin, so Atom`s Structure follows Direction of External

Spin as the Resultant-Spin of every Rim. This is the Why Electrons occupy the Lower-Energy Quantum-Rims. Because Rims are shifted, the shape of Stereo-Slices is a Double Helix , as this happens to DNA structure and is dependent on the number of the Rims. All shapes of DNA can be created from this Gyroscope-Stereo-Slice Mechanism -GSSM- as in Figure 15 . On GSSM , The Periodic Table is now easily drawn

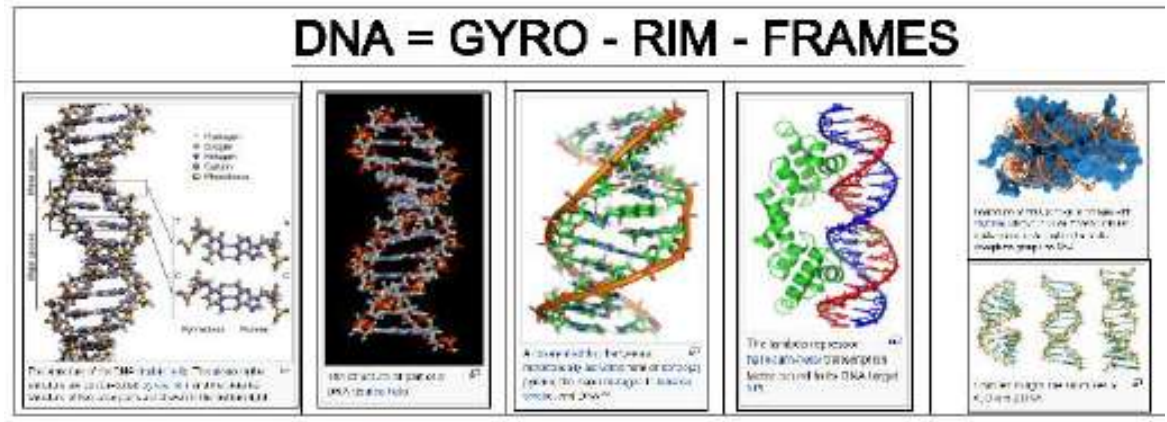

Figure 15.

The different DNA Shapes follow the GSSM Mechanism.

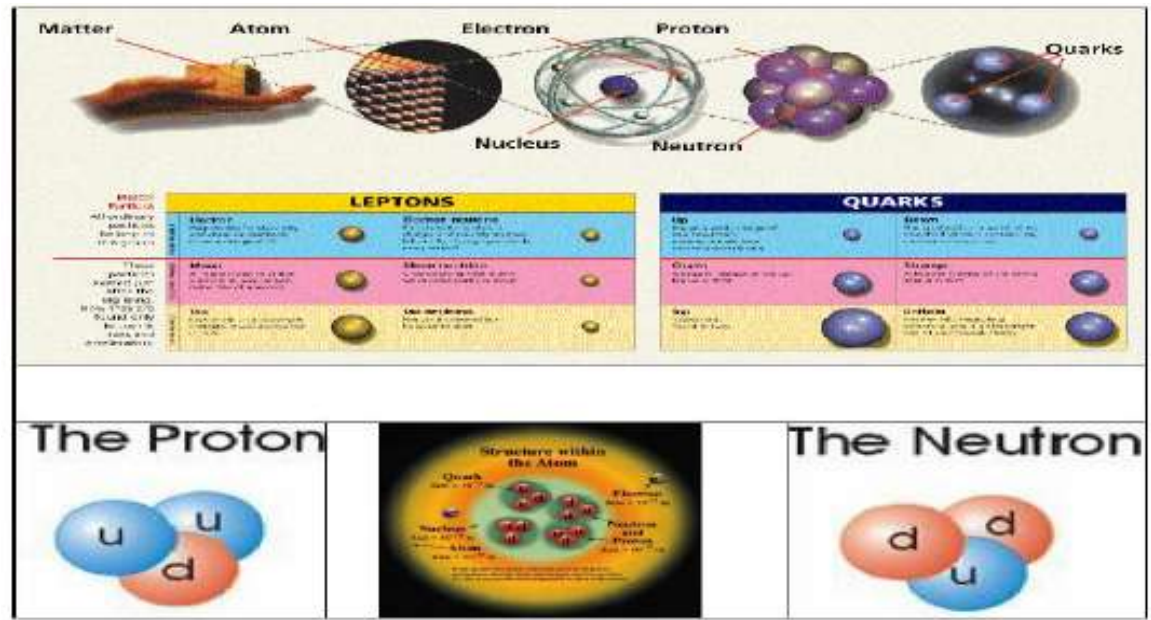

Figure 16.

The Mechanism of , Proton Neutrons from Quarks-Number and Position.

Quarks and all Fundamental Particles in Planck`s Scale are Particles of Gravity-scale.

The Existing Periodic Table Of Elements The Material Geometry and the Origin of Energy-Particles in Periodic System 


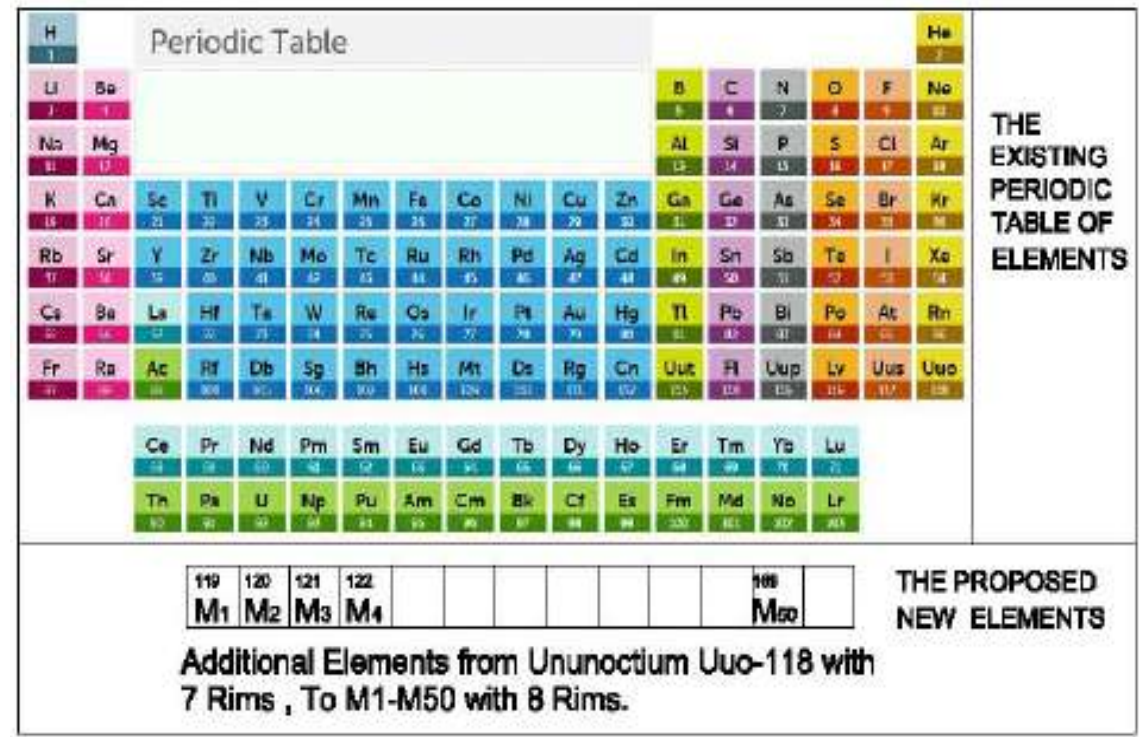

Figure 17

The Periodic Table of Particles with the 118 Elements.

The Proposed New elements become from the completion of the Open-Rim (7) .

The New Rim (8) is consisted of 50 Positions which are filled with Protons. The New is Following Pascal`s-Triangle-Array where Rims are numbers contained in the Prior ones and so then , follow the Positions in each Orbital so for Electrons as the Base for Protons.

Atom Wheel-Rim Protons-Neutrons In Nucleus Electron- Orbital - Positions

1.. $\mathrm{H}$ WR1 $\mathrm{P}=1 \mathrm{~N}=0 \mathrm{E}=11 \rightarrow \mathrm{P} 1$

2.. He WR2-1 $\mathrm{P}=2 \mathrm{~N}=2 \mathrm{E}=22 \rightarrow \mathrm{P} 2$

3.. Li WR2 $\mathrm{P}=3 \mathrm{~N}=4 \mathrm{E}=2+12+8 \rightarrow \mathrm{P} 1+\mathrm{P} 2$

4.. $\mathrm{Be} 2 \mathrm{P}=4 \mathrm{~N}=5 \mathrm{E}=2+2$

5.. Bo $2 \mathrm{P}=5 \mathrm{~N}=6 \mathrm{E}=2+3$

6.. $\mathrm{C} 2 \mathrm{P}=6 \mathrm{~N}=6 \mathrm{E}=2+4$

7.. $\mathrm{N} 2 \mathrm{P}=7 \mathrm{~N}=7 \mathrm{E}=2+5$

8.. $\mathrm{O} 2 \mathrm{P}=8 \mathrm{~N}=8 \mathrm{E}=2+6$

9.. $\mathrm{F} 2 \mathrm{P}=9 \mathrm{~N}=10 \mathrm{E}=2+7$

10.. Ne $2 \mathrm{P}=10 \mathrm{~N}=10 \mathrm{E}=2+88$ Positions

11.. Na WR $3 \mathrm{P}=11 \mathrm{~N}=12 \mathrm{E}=2+8+12+8+8$

12.. $\mathrm{Mg} 3 \mathrm{P}=12 \mathrm{~N}=12 \mathrm{E}=2+8+2$

13.. $\mathrm{Al} 3 \mathrm{P}=13 \mathrm{~N}=14 \mathrm{E}=2+8+3 \rightarrow \mathrm{P} 1+\mathrm{P} 2+\mathrm{P} 2=\mathrm{P} 1+2 \mathrm{P} 2$

14.. Si $3 \mathrm{P}=14 \mathrm{~N}=14 \mathrm{E}=2+8+4$

15.. $\mathrm{P} 3 \mathrm{P}=15 \mathrm{~N}=16 \mathrm{E}=2+8+5$

16.. $\mathrm{S} 3 \mathrm{P}=16 \mathrm{~N}=16 \mathrm{E}=2+8+6$

17.. $\mathrm{Cl} 3 \mathrm{P}=17 \mathrm{~N}=18 \mathrm{E}=2+8+7$

18.. Ar $3 \mathrm{P}=18 \mathrm{~N}=22 \mathrm{E}=2+8+88$ Positions

19.. K WR4-1 $\mathrm{P}=19 \mathrm{~N}=20 \mathrm{E}=2+8+8+12+8+18+8$

20.. $\mathrm{Ca} 4 \mathrm{P}=20 \mathrm{~N}=20 \mathrm{E}=2+8+8+1$

21.. Sc $4 \mathrm{P}=21 \mathrm{~N}=24 \mathrm{E}=2+8+9+2 \rightarrow \mathrm{P} 1+2 \mathrm{P} 2+\mathrm{P} 3$

22.. Ti $4 \mathrm{P}=22 \mathrm{~N}=26 \mathrm{E}=2+8+10+2$

23.. Va $4 \mathrm{P}=23 \mathrm{~N}=28 \mathrm{E}=2+8+11+2$

24.. $\mathrm{Cr} 4 \mathrm{P}=24 \mathrm{~N}=28 \mathrm{E}=2+8+13+1$

25.. $\mathrm{Mn} 4 \mathrm{P}=25 \mathrm{~N}=30 \mathrm{E}=2+8+13+2$

26.. $\mathrm{Fe} 4 \mathrm{P}=26 \mathrm{~N}=30 \mathrm{E}=2+8+14+2$

27.. $\mathrm{Co} 4 \mathrm{P}=27 \mathrm{~N}=32 \mathrm{E}=2+8+15+2$

28.. Ni $4 \mathrm{P}=28 \mathrm{~N}=31 \mathrm{E}=2+8+16+2$

29.. $\mathrm{Cu} 4 \mathrm{P}=29 \mathrm{~N}=35 \mathrm{E}=2+8+18+1$

30.. $\mathrm{Zn} 4 \mathrm{P}=30 \mathrm{~N}=35 \mathrm{E}=2+8+18+2$ 
31.. Ga $4 \mathrm{P}=31 \mathrm{~N}=39 \mathrm{E}=2+8+18+3$

32.. Ge $4 \mathrm{P}=32 \mathrm{~N}=41 \mathrm{E}=2+8+18+4$

33.. As $4 \mathrm{P}=33 \mathrm{~N}=42 \mathrm{E}=2+8+18+5$

34.. $\mathrm{Se} 4 \mathrm{P}=34 \mathrm{~N}=45 \mathrm{E}=2+8+18+6$

35.. $\mathrm{Br} 4 \mathrm{P}=35 \mathrm{~N}=45 \mathrm{E}=2+8+18+7$

36.. $\mathrm{Kr} 4 \mathrm{P}=36 \mathrm{~N}=48 \mathrm{E}=2+8+18+818$ Positions

37.. $\mathrm{Rb}$ WR5 $\mathrm{P}=37 \mathrm{~N}=48 \mathrm{E}=2+8+18+8+12+8+18+18+8$

38.. $\mathrm{Sr} 5 \mathrm{P}=38 \mathrm{~N}=50 \mathrm{E}=2+8+18+8+2$

39.. $\mathrm{Y} 5 \mathrm{P}=39 \mathrm{~N}=50 \mathrm{E}=2+8+18+9+2 \rightarrow \mathrm{P} 1+2 \mathrm{P} 2+2 \mathrm{P} 3$

40.. $\mathrm{Zr} 5 \mathrm{P}=40 \mathrm{~N}=51 \mathrm{E}=2+8+18+10+2$

41.. $\mathrm{Nb} 5 \mathrm{P}=41 \mathrm{~N}=52 \mathrm{E}=2+8+18+12+1$

42.. Mo $5 \mathrm{P}=42 \mathrm{~N}=54 \mathrm{E}=2+8+18+13+1$

43.. Tc $5 \mathrm{P}=43 \mathrm{~N}=55 \mathrm{E}=2+8+18+14+1$

44.. $\mathrm{Ru} 5 \mathrm{P}=44 \mathrm{~N}=57 \mathrm{E}=2+8+18+15+1$

45.. Rh $5 \mathrm{P}=45 \mathrm{~N}=58 \mathrm{E}=2+8+18+16+1$

46.. $\mathrm{Pd} 5 \mathrm{P}=46 \mathrm{~N}=60 \mathrm{E}=2+8+18+18+0$

47.. $\mathrm{Ag} 5 \mathrm{P}=47 \mathrm{~N}=61 \mathrm{E}=2+8+18+18+1$

48.. $\mathrm{Cd} 5 \mathrm{P}=48 \mathrm{~N}=64 \mathrm{E}=2+8+18+18+2$

49.. In $5 \mathrm{P}=49 \mathrm{~N}=66 \mathrm{E}=2+8+18+18+3$

50.. Sn $5 \mathrm{P}=50 \mathrm{~N}=69 \mathrm{E}=2+8+18+18+4$

51.. $\mathrm{Sb} 5 \mathrm{P}=51 \mathrm{~N}=71 \mathrm{E}=2+8+18+18+5$

52.. Te $5 \mathrm{P}=52 \mathrm{~N}=76 \mathrm{E}=2+8+18+18+6$

53.. I $5 \mathrm{P}=53 \mathrm{~N}=74 \mathrm{E}=2+8+18+18+7$

54.. Xe $5 \mathrm{P}=54 \mathrm{~N}=77 \mathrm{E}=2+8+18+18+818$ Positions

55.. Cs WR6-1 P $=55 \mathrm{~N}=78 \mathrm{E}=2+8+18+18+8+12+8+18+32+18+8$

56.. Ba $6 \mathrm{P}=56 \mathrm{~N}=81 \mathrm{E}=2+8+18+18+8+2$

$57 . . \mathrm{La} 6 \mathrm{P}=57 \mathrm{~N}=82 \mathrm{E}=2+8+18+18+9+1 \rightarrow \mathrm{P} 1+2 \mathrm{P} 2+2 \mathrm{P} 3+\mathrm{P} 4$

58. $\mathrm{Ce} 6 \mathrm{P}=58 \mathrm{~N}=82 \mathrm{E}=2+8+18+20+8+2$

59.. $\operatorname{Pr} 6 \mathrm{P}=59 \mathrm{~N}=82 \mathrm{E}=2+8+18+21+8+2$

60.. $\mathrm{Nd} 6 \mathrm{P}=60 \mathrm{~N}=84 \mathrm{E}=2+8+18+22+8+2$

61.. $\mathrm{Pm} 6 \mathrm{P}=61 \mathrm{~N}=84 \mathrm{E}=2+8+18+23+8+2$

62.. $\mathrm{Sm} 6 \mathrm{P}=62 \mathrm{~N}=88 \mathrm{E}=2+8+18+24+8+2$

63.. Eu $6 \mathrm{P}=63 \mathrm{~N}=89 \mathrm{E}=2+8+18+25+8+1$

64.. Gd $6 \mathrm{P}=64 \mathrm{~N}=93 \mathrm{E}=2+8+18+25+9+2$

65.. $\mathrm{Tb} 6 \mathrm{P}=65 \mathrm{~N}=94 \mathrm{E}=2+8+18+27+8+2$

66.. Dy $6 \mathrm{P}=66 \mathrm{~N}=97 \mathrm{E}=2+8+18+28+8+2$

67. Ho $6 \mathrm{P}=67 \mathrm{~N}=98 \mathrm{E}=2+8+18+29+8+2$

68.. $\mathrm{E}$ r $6 \mathrm{P}=68 \mathrm{~N}=99 \mathrm{E}=2+8+18+30+8+2$

69.. $\mathrm{Tm} 6 \mathrm{P}=69 \mathrm{~N}=100 \mathrm{E}=2+8+18+31+8+2$

70.. Yb $6 \mathrm{P}=70 \mathrm{~N}=103 \mathrm{E}=2+8+18+32+8+2$

71.. Lu $6 \mathrm{P}=71 \mathrm{~N}=104 \mathrm{E}=2+8+18+32+9+2$

72.. Hf $6 \mathrm{P}=72 \mathrm{~N}=106 \mathrm{E}=2+8+18+32+10+2$

73. Ta $6 \mathrm{P}=73 \mathrm{~N}=108 \mathrm{E}=2+8+18+32+11+2$

74.. $\mathrm{W} 6 \mathrm{P}=74 \mathrm{~N}=110 \mathrm{E}=2+8+18+32+12+2$

75.. $\operatorname{Re} 6 \mathrm{P}=75 \mathrm{~N}=111 \mathrm{E}=2+8+18+32+13+2$

76.. Os $6 \mathrm{P}=76 \mathrm{~N}=114 \mathrm{E}=2+8+18+32+14+2$

77.. Ir $6 \mathrm{P}=77 \mathrm{~N}=115 \mathrm{E}=2+8+18+32+15+2$

78.. Pt $6 \mathrm{P}=78 \mathrm{~N}=117 \mathrm{E}=2+8+18+32+16+2$

79.. Au $6 \mathrm{P}=79 \mathrm{~N}=118 \mathrm{E}=2+8+18+32+17+2$

80.. $\mathrm{Hg} 6 \mathrm{P}=80 \mathrm{~N}=121 \mathrm{E}=2+8+18+32+18+2$

81.. Tl $6 \mathrm{P}=81 \mathrm{~N}=123 \mathrm{E}=2+8+18+32+18+3$

82.. $\mathrm{Pb} 6 \mathrm{P}=82 \mathrm{~N}=126 \mathrm{E}=2+8+18+32+18+4$

83.. $\mathrm{Bi} 6 \mathrm{P}=83 \mathrm{~N}=126 \mathrm{E}=2+8+18+32+18+5$

84.. Po $6 \mathrm{P}=84 \mathrm{~N}=125 \mathrm{E}=2+8+18+32+18+6$

85.. At $6 \mathrm{P}=85 \mathrm{~N}=125 \mathrm{E}=2+8+18+32+18+7$

86.. Rn $6 \mathrm{P}=86 \mathrm{~N}=136 \mathrm{E}=2+8+18+32+18+832$ Positions 
87.. Fr WR7 $\mathrm{P}=87 \mathrm{~N}=136 \mathrm{E}=2+8+18+32+18+82+8+18+32+32+18+8$

88.. Ra $7 \mathrm{P}=88 \mathrm{~N}=138 \mathrm{E}=2+8+18+32+18+8+2$

89.. Ac $7 \mathrm{P}=89 \mathrm{~N}=138 \mathrm{E}=2+8+18+32+18+9+2 \rightarrow \mathrm{P} 1+2 \mathrm{P} 2+2 \mathrm{P} 3+2 \mathrm{P} 4$

90.. Th $7 \mathrm{P}=90 \mathrm{~N}=142 \mathrm{E}=2+8+18+32+18+10+2$

91.. $\mathrm{Pa} 7 \mathrm{P}=91 \mathrm{~N}=140 \mathrm{E}=2+8+18+32+20+9+0$

92.. U $7 \mathrm{P}=92 \mathrm{~N}=146 \mathrm{E}=2+8+18+32+21+9+0$

93.. $\mathrm{Np} 7 \mathrm{P}=93 \mathrm{~N}=144 \mathrm{E}=2+8+18+32+23+8+0$

94.. Pu $7 \mathrm{P}=94 \mathrm{~N}=150 \mathrm{E}=2+8+18+32+24+8+0$

95.. Am $7 \mathrm{P}=95 \mathrm{~N}=148 \mathrm{E}=2+8+18+32+25+8+0$

96.. $\mathrm{Cm} 7 \mathrm{P}=96 \mathrm{~N}=151 \mathrm{E}=2+8+18+32+25+9+0$

97.. $\mathrm{Bk} 7 \mathrm{P}=97 \mathrm{~N}=150 \mathrm{E}=2+8+18+32+26+9+0$

98.. Cf $7 \mathrm{P}=98 \mathrm{~N}=153 \mathrm{E}=2+8+18+32+28+8+0$

99.. Es $7 \mathrm{P}=99 \mathrm{~N}=153 \mathrm{E}=2+8+18+32+29+8+0$

100.. Fm $7 \mathrm{P}=100 \mathrm{~N}=157 \mathrm{E}=2+8+18+32+30+8+0$

101.. $\mathrm{Md} 7 \mathrm{P}=101 \mathrm{~N}=157 \mathrm{E}=2+8+18+32+31+8+0$

102.. No $7 \mathrm{P}=102 \mathrm{~N}=157 \mathrm{E}=2+8+18+32+32+8+0$

103.. $\mathrm{Lr} 7 \mathrm{P}=103 \mathrm{~N}=159 \mathrm{E}=2+8+18+32+32+9+0$

104.. $\mathrm{Rf} 7 \mathrm{P}=104 \mathrm{~N}=157 \mathrm{E}=2+8+18+32+32+10+0$

105.. Db $7 \mathrm{P}=105 \mathrm{~N}=157 \mathrm{E}=2+8+18+32+32+11+0$

106.. $\mathrm{Sg} 7 \mathrm{P}=106 \mathrm{~N}=157 \mathrm{E}=2+8+18+32+32+12+0$

107.. $\mathrm{Bh} 7 \mathrm{P}=107 \mathrm{~N}=155 \mathrm{E}=2+8+18+32+32+13+0$

108.. Hs $7 \mathrm{P}=108 \mathrm{~N}=157 \mathrm{E}=2+8+18+32+32+14+0$

109.. Mt $7 \mathrm{P}=109 \mathrm{~N}=157 \mathrm{E}=2+8+18+32+32+15+0$

.. $\mathrm{Xx} 7 \mathrm{P}=109 \mathrm{~N}=159 \mathrm{E}=2+8+18+32+32+16+0$

110.. Ds $7 \mathrm{P}=110 \mathrm{~N}=157 \mathrm{E}=2+8+18+32+32+17+0$

111.. $\operatorname{Rg} 7 \mathrm{P}=111 \mathrm{~N}=161 \mathrm{E}=2+8+18+32+32+18+0$

112.. $\mathrm{Cn} 7 \mathrm{P}=112 \mathrm{~N}=165 \mathrm{E}=2+8+18+32+32+18+1$

113.. Uut $7 \mathrm{P}=113 \mathrm{~N}=169 \mathrm{E}=2+8+18+32+32+18+2$

114.. $\mathrm{Fl} 7 \mathrm{P}=114 \mathrm{~N}=173 \mathrm{E}=2+8+18+32+30+18+3$

115.. Uup $7 \mathrm{P}=115 \mathrm{~N}=177 \mathrm{E}=2+8+18+32+30+18+4$

116.. $\mathrm{Lv} 7 \mathrm{P}=116 \mathrm{~N}=181 \mathrm{E}=2+8+18+32+32+18+5$

117.. Uus $7 \mathrm{P}=117 \mathrm{~N}=185 \mathrm{E}=2+8+18+32+32+18+6$

118.. Uuo $7 \mathrm{P}=118 \mathrm{~N}=189 \mathrm{E}=2+8+18+32+32+18+7$

119.. M1 WR8 $\mathrm{P}=119 \mathrm{~N}=193 \mathrm{E}=2+8+18+32+32+18+832$ Positions

120.. $\mathrm{M} 28 \mathrm{P}=120 \mathrm{~N}=197 \mathrm{E}=2+8+18+32+50+32+18+8+12+8+18+32+50+32+18+8$

121.. $\mathrm{M} 38 \mathrm{P}=121 \mathrm{~N}=201 \mathrm{E}=2+8+18+32+50+32+18+8+2$

122.. M4 $8 \mathrm{P}=122 \mathrm{~N}=205 \mathrm{E}=2+8+18+32+50+32+18+8+3 \rightarrow \mathrm{P} 1+2 \mathrm{P} 2+2 \mathrm{P} 3+2 \mathrm{P} 4+\mathrm{R} 5$

WHEEL-RIMS DEPOSITED IN ATOMS . THE SERIES-RIMS IN ATOMS [ Dependent on number of Protons ]

W-Rim.1. Contains 1 Positions $1 \equiv \mathrm{P} 1$

W-Rim.2. Contains 2 Positions $1+1=2 \equiv \mathrm{P} 1+\mathrm{P} 1$

W-Rim.3. Contains 10 Positions $2+8 \equiv 2 \mathrm{P} 1+\mathrm{P} 2$

W-Rim.4. Contains 18 Positions $2+8+8 \equiv 2 \mathrm{P} 1+2 \mathrm{P} 2$

W-Rim.5. Contains 36 Positions $2+8+18+8 \equiv 2 \mathrm{P} 1+2 \mathrm{P} 2+\mathrm{P} 3$

W-Rim.6. Contains 54 Positions $2+8+18+18+8 \equiv 2 \mathrm{P} 1+2 \mathrm{P} 2+2 \mathrm{P} 3$

W-Rim.7. Contains 86 Positions $2+8+18+32+18+8 \equiv 2 \mathrm{P} 1+2 \mathrm{P} 2+2 \mathrm{P} 3+\mathrm{P} 4$

W-Rim.8. Contains 118 Positions $2+8+18+32+32+18+8 \equiv 2 \mathrm{P} 1+2 \mathrm{P} 2+2 \mathrm{P} 3+2 \mathrm{P} 4$

W-Rim.9. Contains 168 Positions $2+8+18+32+50+32+18+8 \equiv 2 \mathrm{P} 1+2 \mathrm{P} 2+2 \mathrm{P} 3+2 \mathrm{P} 4+\mathrm{P} 5$

W-Rim.10. Contains 218 Positions $2+8+18+32+50+50+32+18+8 \equiv 2 \mathrm{P} 1+2 \mathrm{P} 2+2 \mathrm{P} 3+2 \mathrm{P} 4+2 \mathrm{P} 5$

W-Rim.11. Contains 290 Positions $2+8+18+32+50+72+50+32+18+8 \equiv 2 \mathrm{P} 1+2 \mathrm{P} 2+2 \mathrm{P} 3+2 \mathrm{P} 4+2 \mathrm{P} 5+\mathrm{P} 6$

W-Rim. P. Contains 2. $[2.12+2.22+2.32+2.42+2.52+2.62+2 . \mathrm{P} 2]=2 .[\mathrm{M} 12]+4[\mathrm{M} 12+\mathrm{M} 22+\mathrm{M} 32+\mathrm{M} 42+\ldots \mathrm{Mn} 2]$ Positions

Because each Atom contains the Prior-One and since each Rim contains also the Prior-One as Gyro, therefore follow Pascal`s - Triangle array of the binomial coefficients where, in every Container exist the prior and a New 
one depended on The-Space-Positions. If Space-Positions are less of those of , -1 , Space Positions, then is repeated the Prior, differently is added the new as in diagram.

Using Summation of series one can easily find the Number-Positions of each Atom.

Extending this Geometrical-Logic-Position, then from infinite Permutations and Combinations of Protons $=$ Positives, and Electrons $=$ Negatives, is possible to form all existing Atoms and to define all the next .

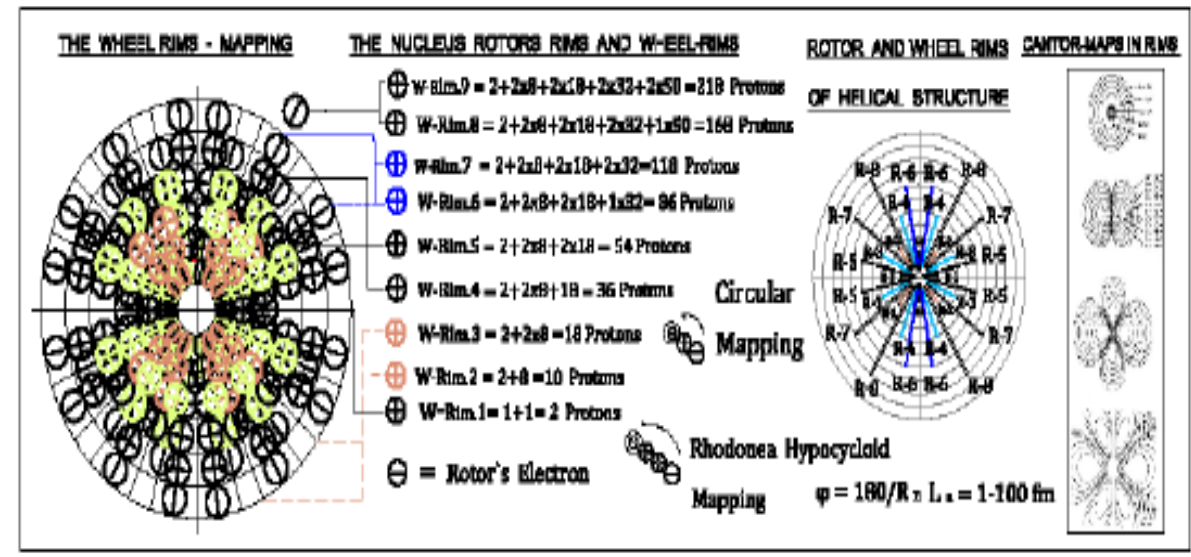

Figure. 18.

The Nucleus Rotors of Atoms follow Pascal`s-Triangle array and Rhodonea Hypocycloid curve. Plan (1) shows the Possible Geometrical Positions of Positives , Protons , in any equilibrium structure of MaterialGeometry, when in contact , $\bigoplus \ominus$, and equilibrium is of the two opposite and equal action forces $\mathrm{F}$, - F , on their common point . Because the two Forces, the Glued-Bond, are opposite and are continually acting on this common point, then according to Newton`s third law, This Common Point executes a Circular Motion creating a Wheel-Rim , while contact $\bigoplus \bigoplus \ominus$ executes a Different Motion . Each Wheel-Rim Perpendicularly placed on the Rotor according to $\varphi=180^{\circ} / \mathrm{M}=$ mould , contains Orbitals following Momentum-Stability in where the number of Protons are depended on the Geometry-Space. Plan (2) shows the Gyro-Rims following the DoubleHelix construction with the Outer-Rims tobe filled in Gups with Electrons and be Free for Bonding .

\subsection{Energy in Atoms .}

The Electronic Structure Of Atoms And Molecules, And Energy- Quantization .

Remarks :

1.As prior referred for the First-Rotating-Monad, the common point executes circular motion on the Positive breakage of radius , r. Wheel-Rim is a closed Stereo-Slate-Tube for Positive and Negative breakages, and Free to undergo transverse vibrations, which gives Odd-numbered harmonics only, and simultaneously Open-Tube , which gives both Odd-and-Even numbered harmonics.

2.Energy of Rims or, Energy-Level, become from Rotational-Energy only, therefore issue Mechanical equations of motion, Independently of magnitude as ,

Rotational energy Er = r.m.v ....(1) where $r=$ The radius of rotation , $\mathrm{m}=$ The mass of inner-motion of particle ( it is the reaction

$\mathrm{v}=$ The tangential velocity. to inner motion )

$\mathrm{w}=$ The angular velocity.

$\mathrm{f}=$ The frequency of motion $, \mathrm{v}=\mathrm{w} \mathrm{r}, \mathrm{w}=2 \pi \mathrm{T}=2 \pi \mathrm{f}$ and (1) becomes Er $=\mathrm{vw}(\mathrm{m} . \mathrm{v})=\mathrm{v}^{2} \mathrm{mw}=\mathrm{v}^{2} \mathrm{~m} 2 \pi \mathrm{f}=\mathrm{v}^{2}$ $\mathrm{mf} 12 \pi=\mathrm{v}^{2}[\mathrm{mT} .12 \pi]=\mathrm{v}^{2}[\mathrm{~h} 2 \mathrm{~T}] \rightarrow$ because $\mathrm{mT}=\mathrm{mf}=$ The stored energy in Wheel-Rim for the fundamental frequency fo $=1$, or $\mathrm{T}=1$ and becomes from the relation $\lambda=2 \mathrm{~L}$ of the Stationary - Waves as $\rightarrow$ fo $=v \lambda=v 2 \mathrm{~L}$ i.e.

h.

The stored energy in the First-Rim is $\rightarrow \mathbf{E}_{\mathbf{1}}=\mathbf{v}^{\mathbf{2}}\left[\frac{\mathbf{h}}{2 \pi}\right]$ depending on velocity, $\mathbf{v}$, and Planck`s constant

For a single photon of Red-light with $\lambda=700 \mathrm{~nm}=700.10-9 \mathrm{~m}$, then fundamental frequency fo is , [ $1 \mathrm{~nm}=10-9 \mathrm{~m}, 1 \mathrm{~A}^{\circ}=10-10 \mathrm{~m}$ and $\mathrm{h} / 2 \mathrm{~m}=1,603.10-16 \mathrm{e} . \mathrm{V} \mathrm{s}$, Velocity light is $\left.2,9979.10-8 \mathrm{~m}\right]$, fo $=v \lambda=c \lambda=2,9979.10-8 \mathrm{~m} / 7.10-7=4,2827.10-16$, and the Energy of the first orbit is $\mathrm{E} 1=\mathrm{v}^{2}[\mathrm{~h} 2 \pi]=(2,9979)^{2} .1016 .[1,603.10-16 \mathrm{~h}-\mathrm{bar}]=14,41 \mathrm{eV} . \mathrm{s}$

Energy of the first Slice-Wheel-Rim or Orbital is distributed to orbit Electron, while energy of the second Slice-Wheel-Rim to the couple of permitted Electrons (2e) of the orbital and is ,

$\mathrm{E} 1=\mathrm{v}^{2}[\mathrm{~h} 2 \mathrm{~T}] / \mathrm{R} 1^{2}=1^{2}=1$

$\mathrm{E} 2=\mathrm{v}^{2}[\mathrm{~h} 2 \pi] / \mathrm{R} 2^{2}=2^{2}=4$ and for , c , Space Number, 
$\mathrm{Ec}=\mathrm{v}^{2}[\mathrm{~h} 2 \mathrm{~T}] / \mathrm{Rc}^{2}=\mathrm{Rc}^{2}$

Because any Next-Atom Energy, is equal to Prior + the distributed [ E1Rc ${ }^{2}$. 12.Rc ${ }^{2}$ ] then, Energy of Hydrogen W-Rim.1 E1=E1/12 $=14,41 \mathrm{eV} . \mathrm{s}$

of Helium W-Rim.2 $\quad \mathbf{E}_{2}=\mathrm{E}_{1}+\left[\frac{\mathrm{E} 1}{1} \frac{1}{1.2^{2}}\right] \quad=\mathrm{E}_{1}+\mathrm{E}_{1} / 4$

of Lithium-Rim

$\mathrm{E}_{3}=\mathrm{E}_{1}+\frac{\mathrm{E} 1}{4}+\left[\frac{\mathrm{E} 1}{2^{2}} \frac{1}{2.2^{2}}\right] \quad=\mathrm{E}_{1}+\left(\frac{\mathrm{E} 1}{4}\right) \cdot \frac{1}{8}$

of Beryllium-Rim $\mathrm{E}_{4}=\mathrm{E}_{1}+\left(\frac{\mathrm{E} 1}{4}\right) \cdot \frac{1}{8}+\left[\frac{\mathrm{E} 1}{2^{2}} \frac{1}{2 \cdot 2^{2}}\right]=\mathrm{E}_{1}+\left(\frac{\mathrm{E} 1}{4}\right) \cdot \frac{2}{8}$

of Boron-Rim

$\mathrm{E}_{5}=\mathrm{E}_{1}+\left(\frac{\mathrm{E} 1}{4}\right) \cdot \frac{2}{8}+\left[\frac{\mathrm{E} 1}{2^{2}} \frac{1}{2.2^{2}}\right]=\mathrm{E}_{1}+\left(\frac{\mathrm{E} 1}{4}\right) \cdot \frac{3}{8}$

of Carbon-Rim

$\mathrm{E}_{6}=\mathrm{E}_{1}+\left(\frac{\mathrm{E} 1}{4}\right) \cdot \frac{3}{8}+\left[\frac{\mathrm{E} 1}{2^{2}} \frac{1}{2.2^{2}}\right]=\mathrm{E}_{1}+\left(\frac{\mathrm{E} 1}{4}\right) \cdot \frac{4}{8}$

of Nitrogen-Rim

$\mathrm{E}_{7}=\mathrm{E}_{1}+\left(\frac{\mathrm{E} 1}{4}\right) \cdot \frac{4}{8}+\left[\frac{\mathrm{E} 1}{2^{2}} \frac{1}{2.2^{2}}\right]=\mathrm{E}_{1}+\left(\frac{\mathrm{E} 1}{4}\right) \cdot \frac{5}{8}$

of Oxygen-Rim

$E_{8}=E_{1}+\left(\frac{E 1}{4}\right) \cdot \frac{5}{8}+\left[\frac{E 1}{2^{2}} \frac{1}{2.2^{2}}\right]=E_{1}+\left(\frac{E 1}{4}\right) \cdot \frac{6}{8}$

of Fluoride-Rim

$\mathrm{E}_{9}=\mathrm{E}_{1}+\left(\frac{\mathrm{E} 1}{4}\right) \cdot \frac{6}{8}+\left[\frac{\mathrm{E} 1}{2^{2}} \frac{1}{2.2^{2}}\right]=\mathrm{E}_{1}+\left(\frac{\mathrm{E} 1}{4}\right) \cdot \frac{7}{8}$

of Neon W-Rim.2 $\mathbf{E}_{\mathbf{1 0}}=\mathrm{E}_{1}+\left(\frac{\mathrm{E} 1}{4}\right) \cdot \frac{7}{8}+\left[\frac{\mathrm{E} 1}{2^{2}} \frac{1}{2.2^{2}}\right]=\mathrm{E}_{1}+\left(\frac{\mathrm{E} 1}{4}\right) \cdot \frac{8}{8}=\mathrm{E}_{1}+\left(\frac{\mathrm{E} 1}{4}\right)$

of Sodium-Rim. $3 \quad E_{11}=\left(E_{1}+\frac{E 1}{4}\right)+\left[\frac{E 1}{3^{2}} \frac{1}{2 \cdot 2^{2}}\right]=\left(E_{1}+\frac{E 1}{4}\right)+\left(\frac{E 1}{9}\right) \cdot \frac{1}{8}$

of Magnesium-Rim $E_{12}=\left(E_{1}+\frac{E 1}{4}\right)+\left(\frac{E 1}{9}\right) \cdot \frac{1}{8}+\left[\frac{E 1}{3^{2}} \frac{1}{2 \cdot 2^{2}}\right]=\left(E_{1}+\frac{E 1}{4}\right)+\left(\frac{E 1}{9}\right) \cdot \frac{2}{8}$

of Aluminum-Rim $E_{13}=\left(E_{1}+\frac{E 1}{4}\right)+\left(\frac{E 1}{9}\right) \cdot \frac{2}{8}+\left[\frac{E 1}{3^{2}} \frac{1}{2 \cdot 2^{2}}\right]=\left(E_{1}+\frac{E 1}{4}\right)+\left(\frac{E 1}{9}\right) \cdot \frac{3}{8}$

of Silicon-Rim $\quad E_{14}=\left(E_{1}+\frac{E 1}{4}\right)+\left(\frac{E 1}{9}\right) \cdot \frac{3}{8}+\left[\frac{E 1}{3^{2}} \frac{1}{2 \cdot 2^{2}}\right]=\left(E_{1}+\frac{E 1}{4}\right)+\left(\frac{E 1}{9}\right) \cdot \frac{4}{8}$

of Phosphorus-Rim $E_{15}=\left(E_{1}+\frac{E 1}{4}\right)+\left(\frac{E 1}{9}\right) \cdot \frac{4}{8}+\left[\frac{E 1}{3^{2}} \frac{1}{2 \cdot 2^{2}}\right]=\left(E_{1}+\frac{E 1}{4}\right)+\left(\frac{E 1}{9}\right) \cdot \frac{5}{8}$

of Sulfur-Rim $\quad E_{16}=\left(E_{1}+\frac{E 1}{4}\right)+\left(\frac{E 1}{9}\right) \cdot \frac{5}{8}+\left[\frac{E 1}{3^{2}} \frac{1}{2 \cdot 2^{2}}\right]=\left(E_{1}+\frac{E 1}{4}\right)+\left(\frac{E 1}{9}\right) \cdot \frac{6}{8}$

of Chlorine-Rim $\quad E_{17}=\left(E_{1}+\frac{E 1}{4}\right)+\left(\frac{E 1}{9}\right) \cdot \frac{6}{8}+\left[\frac{E 1}{3^{2}} \frac{1}{2 \cdot 2^{2}}\right]=\left(E_{1}+\frac{E 1}{4}\right)+\left(\frac{E 1}{9}\right) \cdot \frac{7}{8}$

of Argon W-Rim.3 $\mathbf{E}_{\mathbf{1 8}}=\left(E_{1}+\frac{E 1}{4}\right)+\left(\frac{E 1}{9}\right) \cdot \frac{7}{8}+\left[\frac{E 1}{3^{2}} \frac{8}{2 \cdot 2^{2}}\right]=\left(E_{1}+\frac{E 1}{4}\right)+\left(\frac{E 1}{9}\right) \cdot \frac{8}{8}=\left(E_{1}+\frac{E 1}{4}\right)+\left(\frac{E 1}{9}\right)$

of Potassium-Rim.4 $E_{19}=\left(E_{1}+\frac{E 1}{4}+\frac{E 1}{9}\right)+\left[\frac{E 1}{4^{2}} \frac{1}{2.3^{2}}\right]=\left(E_{1}+\frac{E 1}{4}+\frac{E 1}{9}\right)+\left(\frac{E 1}{16}\right) \cdot \frac{01}{18}$

of Calcium-Rim $\quad E_{20}=\left(E_{1}+\frac{E 1}{4}+\frac{E 1}{9}\right)+\left(\frac{E 1}{16}\right) \cdot \frac{01}{18}+\left[\frac{E 1}{4^{2}} \frac{1}{2.3^{2}}\right]=\left(E_{1}+\frac{E 1}{4}+\frac{E 1}{9}\right)+\left(\frac{E 1}{16}\right) \cdot \frac{02}{18}$

of Scandium-Rim $\quad E_{21}=\left(E_{1}+\frac{E 1}{4}+\frac{E 1}{9}\right)+\left(\frac{E 1}{16}\right) \cdot \frac{02}{18}+\left[\frac{E 1}{4^{2}} \frac{1}{2 \cdot 3^{2}}\right]=\left(E_{1}+\frac{E 1}{4}+\frac{E 1}{9}\right)+\left(\frac{E 1}{16}\right) \cdot \frac{03}{18}$

of Titanium-Rim $\quad E_{22}=\left(E_{1}+\frac{E 1}{4}+\frac{E 1}{9}\right)+\left(\frac{E 1}{16}\right) \cdot \frac{03}{18}+\left[\frac{E 1}{4^{2}} \frac{1}{2.3^{2}}\right]=\left(E_{1}+\frac{E 1}{4}+\frac{E 1}{9}\right)+\left(\frac{E 1}{16}\right) \cdot \frac{04}{18}$

of Vanadium-Rim $\quad E_{23}=\left(E_{1}+\frac{E 1}{4}+\frac{E 1}{9}\right)+\left(\frac{E 1}{16}\right) \cdot \frac{04}{18}+\left[\frac{E 1}{4^{2}} \frac{1}{2.3^{2}}\right]=\left(E_{1}+\frac{E 1}{4}+\frac{E 1}{9}\right)+\left(\frac{E 1}{16}\right) \cdot \frac{05}{18}$

of Chromium-Rim $\quad E_{24}=\left(E_{1}+\frac{E 1}{4}+\frac{E 1}{9}\right)+\left(\frac{E 1}{16}\right) \cdot \frac{05}{18}+\left[\frac{E 1}{4^{2}} \frac{1}{2.3^{2}}\right]=\left(E_{1}+\frac{E 1}{4}+\frac{E 1}{9}\right)+\left(\frac{E 1}{16}\right) \cdot \frac{06}{18}$

of Manganese-Rim $E_{25}=\left(E_{1}+\frac{E 1}{4}+\frac{E 1}{9}\right)+\left(\frac{E 1}{16}\right) \cdot \frac{06}{18}+\left[\frac{E 1}{4^{2}} \frac{1}{2 \cdot 3^{2}}\right]=\left(E_{1}+\frac{E 1}{4}+\frac{E 1}{9}\right)+\left(\frac{E 1}{16}\right) \cdot \frac{07}{18}$

of Iron-Rim

$E_{26}=\left(E_{1}+\frac{E 1}{4}+\frac{E 1}{9}\right)+\left(\frac{E 1}{16}\right) \cdot \frac{07}{18}+\left[\frac{E 1}{4^{2}} \frac{1}{2.3^{2}}\right]=\left(E_{1}+\frac{E 1}{4}+\frac{E 1}{9}\right)+\left(\frac{E 1}{16}\right) \cdot \frac{08}{18}$

of Cobalt-Rim

$E_{27}=\left(E_{1}+\frac{E 1}{4}+\frac{E 1}{9}\right)+\left(\frac{E 1}{16}\right) \cdot \frac{08}{18}+\left[\frac{E 1}{4^{2}} \frac{1}{2.3^{2}}\right]=\left(E_{1}+\frac{E 1}{4}+\frac{E 1}{9}\right)+\left(\frac{E 1}{16}\right) \cdot \frac{09}{18}$

of Nickel-Rim

$E_{28}=\left(E_{1}+\frac{E 1}{4}+\frac{E 1}{9}\right)+\left(\frac{E 1}{16}\right) \cdot \frac{09}{18}+\left[\frac{E 1}{4^{2}} \frac{1}{2 \cdot 3^{2}}\right]=\left(E_{1}+\frac{E 1}{4}+\frac{E 1}{9}\right)+\left(\frac{E 1}{16}\right) \cdot \frac{10}{18}$

of Copper-Rim

$E_{29}=\left(E_{1}+\frac{E 1}{4}+\frac{E 1}{9}\right)+\left(\frac{E 1}{16}\right) \cdot \frac{10}{18}+\left[\frac{E 1}{4^{2}} \frac{1}{2 \cdot 3^{2}}\right]=\left(E_{1}+\frac{E 1}{4}+\frac{E 1}{9}\right)+\left(\frac{E 1}{16}\right) \cdot \frac{11}{18}$ 
of Zink-Rim

$$
E_{30}=\left(E_{1}+\frac{E 1}{4}+\frac{E 1}{9}\right)+\left(\frac{E 1}{16}\right) \cdot \frac{11}{18}+\left[\frac{E 1}{4^{2}} \frac{1}{2.3^{2}}\right]=\left(E_{1}+\frac{E 1}{4}+\frac{E 1}{9}\right)+\left(\frac{E 1}{16}\right) \cdot \frac{12}{18}
$$

of Gallium-Rim

$$
E_{31}=\left(E_{1}+\frac{E 1}{4}+\frac{E 1}{9}\right)+\left(\frac{E 1}{16}\right) \cdot \frac{12}{18}+\left[\frac{E 1}{4^{2}} \frac{1}{2 \cdot 3^{2}}\right]=\left(E_{1}+\frac{E 1}{4}+\frac{E 1}{9}\right)+\left(\frac{E 1}{16}\right) \cdot \frac{13}{18}
$$

of Germanium-Rim

$E_{32}=\left(E_{1}+\frac{E 1}{4}+\frac{E 1}{9}\right)+\left(\frac{E 1}{16}\right) \cdot \frac{13}{18}+\left[\frac{E 1}{4^{2}} \frac{1}{2.3^{2}}\right]=\left(E_{1}+\frac{E 1}{4}+\frac{E 1}{9}\right)+\left(\frac{E 1}{16}\right) \cdot \frac{14}{18}$

of Arsenic-Rim

$$
E_{33}=\left(E_{1}+\frac{E 1}{4}+\frac{E 1}{9}\right)+\left(\frac{E 1}{16}\right) \cdot \frac{14}{18}+\left[\frac{E 1}{4^{2}} \frac{1}{2 \cdot 3^{2}}\right]=\left(E_{1}+\frac{E 1}{4}+\frac{E 1}{9}\right)+\left(\frac{E 1}{16}\right) \cdot \frac{15}{18}
$$

of Selenium-Rim

$$
E_{34}=\left(E_{1}+\frac{E 1}{4}+\frac{E 1}{9}\right)+\left(\frac{E 1}{16}\right) \cdot \frac{15}{18}+\left[\frac{E 1}{4^{2}} \frac{1}{2.3^{2}}\right]=\left(E_{1}+\frac{E 1}{4}+\frac{E 1}{9}\right)+\left(\frac{E 1}{16}\right) \cdot \frac{16}{18}
$$

of Bromine-Rim.5

$$
E_{35}=\left(E_{1}+\frac{E 1}{4}+\frac{E 1}{9}\right)+\left(\frac{E 1}{16}\right) \cdot \frac{16}{18}+\left[\frac{E 1}{4^{2}} \frac{1}{2.3^{2}}\right]=\left(E_{1}+\frac{E 1}{4}+\frac{E 1}{9}\right)+\left(\frac{E 1}{16}\right) \cdot \frac{17}{18}
$$

of Krypton W-Rim.4 $\mathbf{E}_{36}=\left(E_{1}+\frac{E 1}{4}+\frac{E 1}{9}\right)+\left(\frac{E 1}{16}\right) \cdot \frac{17}{18}+\left[\frac{E 1}{4^{2}} \frac{1}{2.3^{2}}\right]=\left(E_{1}+\frac{E 1}{4}+\frac{E 1}{9}\right)+\left(\frac{E 1}{16}\right) \cdot \frac{18}{18}=\left(E_{1}+\frac{E 1}{4}+\frac{E 1}{9}+\frac{E 1}{16}\right)$

of Rubidium-Rim.5 $\quad E_{37}=\left(E_{1}+\frac{E 1}{4}+\frac{E 1}{9}+\frac{E 1}{16}\right)+\left[\frac{E 1}{5^{2}} \frac{1}{2 \cdot 3^{2}}\right]=\left(E_{1}+\frac{E 1}{4}+\frac{E 1}{9}+\frac{E 1}{16}\right)+\left(\frac{E 1}{25}\right) \cdot \frac{1}{18}$

of Strontium-Rim $\quad E_{38}=\left(E_{1}+\frac{E 1}{4}+\frac{E 1}{9}+\frac{E 1}{16}\right)+\left(\frac{E 1}{25}\right) \cdot \frac{1}{18}+\left[\frac{E 1}{5^{2}} \frac{1}{2.3^{2}}\right]=\left(E_{1}+\frac{E 1}{4}+\frac{E 1}{9}+\frac{E 1}{16}\right)+\left(\frac{E 1}{25}\right) \cdot \frac{2}{18}$

of Yttrium-Rim $\quad E_{39}=\left(E_{1}+\frac{E 1}{4}+\frac{E 1}{9}+\frac{E 1}{16}\right)+\left(\frac{E 1}{25}\right) \cdot \frac{2}{18}+\left[\frac{E 1}{5^{2}} \frac{1}{2.3^{2}}\right]=\left(E_{1}+\frac{E 1}{4}+\frac{E 1}{9}+\frac{E 1}{16}\right)+\left(\frac{E 1}{25}\right) \cdot \frac{3}{18}$

of Zirconium-Rim $\quad E_{40}=\left(E_{1}+\frac{E 1}{4}+\frac{E 1}{9}+\frac{E 1}{16}\right)+\left(\frac{E 1}{25}\right) \cdot \frac{3}{18}+\left[\frac{E 1}{5^{2}} \frac{1}{2.3^{2}}\right]=\left(E_{1}+\frac{E 1}{4}+\frac{E 1}{9}+\frac{E 1}{16}\right)+\left(\frac{E 1}{25}\right) \cdot \frac{4}{18}$

of Niobium-Rim $\quad E_{41}=\left(E_{1}+\frac{E 1}{4}+\frac{E 1}{9}+\frac{E 1}{16}\right)+\left(\frac{E 1}{25}\right) \cdot \frac{4}{18}+\left[\frac{E 1}{5^{2}} \frac{1}{2 \cdot 3^{2}}\right]=\left(E_{1}+\frac{E 1}{4}+\frac{E 1}{9}+\frac{E 1}{16}\right)+\left(\frac{E 1}{25}\right) \cdot \frac{5}{18}$

of Molybdenum-Rim $E_{42}=\left(E_{1}+\frac{E 1}{4}+\frac{E 1}{9}+\frac{E 1}{16}\right)+\left(\frac{E 1}{25}\right) \cdot \frac{5}{18}+\left[\frac{E 1}{5^{2}} \frac{1}{2.3^{2}}\right]=\left(E_{1}+\frac{E 1}{4}+\frac{E 1}{9}+\frac{E 1}{16}\right)+\left(\frac{E 1}{25}\right) \cdot \frac{6}{18}$

of Technetium-Rim $E_{43}=\left(E_{1}+\frac{E 1}{4}+\frac{E 1}{9}+\frac{E 1}{16}\right)+\left(\frac{E 1}{25}\right) \cdot \frac{6}{18}+\left[\frac{E 1}{5^{2}} \frac{1}{2.3^{2}}\right]=\left(E_{1}+\frac{E 1}{4}+\frac{E 1}{9}+\frac{E 1}{16}\right)+\left(\frac{E 1}{25}\right) \cdot \frac{7}{18}$

of Ruthenium-Rim $\quad E_{44}=\left(E_{1}+\frac{E 1}{4}+\frac{E 1}{9}+\frac{E 1}{16}\right)+\left(\frac{E 1}{25}\right) \cdot \frac{7}{18}+\left[\frac{E 1}{5^{2}} \frac{1}{2 \cdot 3^{2}}\right]=\left(E_{1}+\frac{E 1}{4}+\frac{E 1}{9}+\frac{E 1}{16}\right)+\left(\frac{E 1}{25}\right) \cdot \frac{8}{18}$

of Rhodium-Rim $\quad E_{45}=\left(E_{1}+\frac{E 1}{4}+\frac{E 1}{9}+\frac{E 1}{16}\right)+\left(\frac{E 1}{25}\right) \cdot \frac{8}{18}+\left[\frac{E 1}{5^{2}} \frac{1}{2.3^{2}}\right]=\left(E_{1}+\frac{E 1}{4}+\frac{E 1}{9}+\frac{E 1}{16}\right)+\left(\frac{E 1}{25}\right) \cdot \frac{9}{18}$

of Palladium-Rim $\quad E_{46}=\left(E_{1}+\frac{E 1}{4}+\frac{E 1}{9}+\frac{E 1}{16}\right)+\left(\frac{E 1}{25}\right) \cdot \frac{9}{18}+\left[\frac{E 1}{5^{2}} \frac{1}{2 \cdot 3^{2}}\right]=\left(E_{1}+\frac{E 1}{4}+\frac{E 1}{9}+\frac{E 1}{16}\right)+\left(\frac{E 1}{25}\right) \cdot \frac{10}{18}$

of Silver-Rim

$$
E_{47}=\left(E_{1}+\frac{E 1}{4}+\frac{E 1}{9}+\frac{E 1}{16}\right)+\left(\frac{E 1}{25}\right) \cdot \frac{10}{18}+\left[\frac{E 1}{5^{2}} \frac{1}{2 \cdot 3^{2}}\right]=\left(E_{1}+\frac{E 1}{4}+\frac{E 1}{9}+\frac{E 1}{16}\right)+\left(\frac{E 1}{25}\right) \cdot \frac{11}{18}
$$

of Cadmium-Rim

$E_{48}=\left(E_{1}+\frac{E 1}{4}+\frac{E 1}{9}+\frac{E 1}{16}\right)+\left(\frac{E 1}{25}\right) \cdot \frac{11}{18}+\left[\frac{E 1}{5^{2}} \frac{1}{2.3^{2}}\right]=\left(E_{1}+\frac{E 1}{4}+\frac{E 1}{9}+\frac{E 1}{16}\right)+\left(\frac{E 1}{25}\right) \cdot \frac{12}{18}$

of Indium-Rim

$$
E_{49}=\left(E_{1}+\frac{E 1}{4}+\frac{E 1}{9}+\frac{E 1}{16}\right)+\left(\frac{E 1}{25}\right) \cdot \frac{12}{18}+\left[\frac{E 1}{5^{2}} \frac{1}{2.3^{2}}\right]=\left(E_{1}+\frac{E 1}{4}+\frac{E 1}{9}+\frac{E 1}{16}\right)+\left(\frac{E 1}{25}\right) \cdot \frac{13}{18}
$$

of Tim-Rim

$$
E_{50}=\left(E_{1}+\frac{E 1}{4}+\frac{E 1}{9}+\frac{E 1}{16}\right)+\left(\frac{E 1}{25}\right) \cdot \frac{13}{18}+\left[\frac{E 1}{5^{2}} \frac{1}{2 \cdot 3^{2}}\right]=\left(E_{1}+\frac{E 1}{4}+\frac{E 1}{9}+\frac{E 1}{16}\right)+\left(\frac{E 1}{25}\right) \cdot \frac{14}{18}
$$

of Antimony-Rim $\quad E_{51}=\left(E_{1}+\frac{E 1}{4}+\frac{E 1}{9}+\frac{E 1}{16}\right)+\left(\frac{E 1}{25}\right) \cdot \frac{14}{18}+\left[\frac{E 1}{5^{2}} \frac{1}{2.3^{2}}\right]=\left(E_{1}+\frac{E 1}{4}+\frac{E 1}{9}+\frac{E 1}{16}\right)+\left(\frac{E 1}{25}\right) \cdot \frac{15}{18}$

of Tellurium-Rim $\quad E_{52}=\left(E_{1}+\frac{E 1}{4}+\frac{E 1}{9}+\frac{E 1}{16}\right)+\left(\frac{E 1}{25}\right) \cdot \frac{15}{18}+\left[\frac{E 1}{5^{2}} \frac{1}{2 \cdot 3^{2}}\right]=\left(E_{1}+\frac{E 1}{4}+\frac{E 1}{9}+\frac{E 1}{16}\right)+\left(\frac{E 1}{25}\right) \cdot \frac{16}{18}$

of Iodine-Rim.5

$$
E_{53}=\left(E_{1}+\frac{E 1}{4}+\frac{E 1}{9}+\frac{E 1}{16}\right)+\left(\frac{E 1}{25}\right) \cdot \frac{16}{18}+\left[\frac{E 1}{5^{2}} \frac{1}{2 \cdot 3^{2}}\right]=\left(E_{1}+\frac{E 1}{4}+\frac{E 1}{9}+\frac{E 1}{16}\right)+\left(\frac{E 1}{25}\right) \cdot \frac{17}{18}
$$

of Xenon W-Rim.5 $\mathbf{E}_{\mathbf{5 4}}=\left(\mathrm{E}_{1}+\frac{\mathrm{E} 1}{4}+\frac{\mathrm{E} 1}{9}+\frac{\mathrm{E} 1}{16}\right)+\left(\frac{\mathrm{E} 1}{25}\right) \frac{17}{18}+\left[\frac{\mathrm{E} 1}{5^{2}} \frac{1}{2.3^{2}}\right]=\left[\mathrm{E}_{1}+\frac{\mathrm{E} 1}{4}+\frac{\mathrm{E} 1}{9}+\frac{\mathrm{E} 1}{16}\right]+\left(\frac{\mathrm{E} 1}{25}\right) \cdot \frac{18}{18}=\left[\mathrm{E}_{1}+\frac{\mathrm{E} 1}{4}+\frac{\mathrm{E} 1}{9}+\frac{\mathrm{E} 1}{16}+\frac{\mathrm{E} 1}{25}\right]$

of Cesium-Rim.6

$$
E_{55}=\left[E_{1}+\frac{E 1}{4}+\frac{E 1}{9}+\frac{E 1}{16}+\frac{E 1}{25}\right]+\left(\frac{E 1}{6^{2}} \frac{1}{2 \cdot 4^{2}}\right)=\left[E_{1}+\frac{E 1}{4}+\frac{E 1}{9}+\frac{E 1}{16}+\frac{E 1}{25}\right]+\left(\frac{E 1}{36}\right) \cdot \frac{1}{32}
$$

of Barium-Rim

$$
E_{56}=\left[E_{1}+\frac{E 1}{4}+\frac{E 1}{9}+\frac{E 1}{16}+\frac{E 1}{25}\right]+\left(\frac{E 1}{6^{2}} \frac{1}{2.4^{2}}\right)=\left[E_{1}+\frac{E 1}{4}+\frac{E 1}{9}+\frac{E 1}{16}+\frac{E 1}{25}\right]+\left(\frac{E 1}{36}\right) \cdot \frac{2}{32}
$$

of Lanthanum-Rim

$E_{57}=\left[E_{1}+\frac{E 1}{4}+\frac{E 1}{9}+\frac{E 1}{16}+\frac{E 1}{25}\right]+\left(\frac{E 1}{36} \frac{2}{32}\right)+\left[\frac{E 1}{6^{2}} \frac{1}{2.4^{2}}\right]=\left[E_{1}+\frac{E 1}{4}+\frac{E 1}{9}+\frac{E 1}{16}+\frac{E 1}{25}\right]+\left(\frac{E 1}{36}\right) \cdot \frac{3}{32}$

of Cesium-Rim

$$
E_{58}=\left[E_{1}+\frac{E 1}{4}+\frac{E 1}{9}+\frac{E 1}{16}+\frac{E 1}{25}\right]+\left(\frac{E 1}{36} \frac{3}{32}\right)+\left[\frac{E 1}{6^{2}} \frac{1}{2.4^{2}}\right]=\left[E_{1}+\frac{E 1}{4}+\frac{E 1}{9}+\frac{E 1}{16}+\frac{E 1}{25}\right]+\left(\frac{E 1}{36}\right) \cdot \frac{4}{32}
$$

of Praseodymium-Rim $E_{59}=\left[E_{1}+\frac{E 1}{4}+\frac{E 1}{9}+\frac{E 1}{16}+\frac{E 1}{25}\right]+\left(\frac{E 1}{36} \frac{4}{32}\right)+\left[\frac{E 1}{6^{2}} \frac{1}{2 \cdot 4^{2}}\right]=\left[E_{1}+\frac{E 1}{4}+\frac{E 1}{9}+\frac{E 1}{16}+\frac{E 1}{25}\right]+\left(\frac{E 1}{36}\right) \cdot \frac{5}{32}$ 
of Neodymium-Rim $\quad E_{60}=\left[E_{1}+\frac{E 1}{4}+\frac{E 1}{9}+\frac{E 1}{16}+\frac{E 1}{25}\right]+\left(\frac{E 1}{36} \frac{5}{32}\right)+\left[\frac{E 1}{6^{2}} \frac{1}{2.4^{2}}\right]=\left[E_{1}+\frac{E 1}{4}+\frac{E 1}{9}+\frac{E 1}{16}+\frac{E 1}{25}\right]+\left(\frac{E 1}{36}\right) \cdot \frac{6}{32}$ of Promethium-Rim $E_{61}=\left[E_{1}+\frac{E 1}{4}+\frac{E 1}{9}+\frac{E 1}{16}+\frac{E 1}{25}\right]+\left(\frac{E 1}{36} \frac{6}{32}\right)+\left[\frac{E 1}{6^{2}} \frac{1}{2.4^{2}}\right]=\left[E_{1}+\frac{E 1}{4}+\frac{E 1}{9}+\frac{E 1}{16}+\frac{E 1}{25}\right]+\left(\frac{E 1}{36}\right) \cdot \frac{7}{32}$ of Samarium-Rim $\quad E_{62}=\left[E_{1}+\frac{E 1}{4}+\frac{E 1}{9}+\frac{E 1}{16}+\frac{E 1}{25}\right]+\left(\frac{E 1}{36} \frac{7}{32}\right)+\left[\frac{E 1}{6^{2}} \frac{1}{2.4^{2}}\right]=\left[E_{1}+\frac{E 1}{4}+\frac{E 1}{9}+\frac{E 1}{16}+\frac{E 1}{25}\right]+\left(\frac{E 1}{36}\right) \cdot \frac{8}{32}$ of Europium-Rim $\mathrm{E}_{63}=\left[\mathrm{E}_{1}+\frac{\mathrm{E} 1}{4}+\frac{\mathrm{E} 1}{9}+\frac{\mathrm{E} 1}{16}+\frac{\mathrm{E} 1}{25}\right]+\left(\frac{\mathrm{E} 1}{36} \frac{8}{32}\right)+\left[\frac{\mathrm{E} 1}{6^{2}} \frac{1}{2.4^{2}}\right]=\left[\mathrm{E}_{1}+\frac{\mathrm{E} 1}{4}+\frac{\mathrm{E} 1}{9}+\frac{\mathrm{E} 1}{16}+\frac{\mathrm{E} 1}{25}\right]+\left(\frac{\mathrm{E} 1}{36}\right) \cdot \frac{9}{32}$ of Gadolinium-Rim $\quad E_{64}=\left[E_{1}+\frac{E 1}{4}+\frac{E 1}{9}+\frac{E 1}{16}+\frac{E 1}{25}\right]+\left(\frac{E 1}{36} \frac{9}{32}\right)+\left[\frac{E 1}{6^{2}} \frac{1}{2.4^{2}}\right]=\left[E_{1}+\frac{E 1}{4}+\frac{E 1}{9}+\frac{E 1}{16}+\frac{E 1}{25}\right]+\left(\frac{E 1}{36}\right) \cdot \frac{10}{32}$ of Terbium-Rim $\quad E_{65}=\left[E_{1}+\frac{E 1}{4}+\frac{E 1}{9}+\frac{E 1}{16}+\frac{E 1}{25}\right]+\left(\frac{E 1}{36} \frac{10}{32}\right)+\left[\frac{E 1}{6^{2}} \frac{1}{2.4^{2}}\right]=\left[E_{1}+\frac{E 1}{4}+\frac{E 1}{9}+\frac{E 1}{16}+\frac{E 1}{25}\right]+\left(\frac{E 1}{36}\right) \cdot \frac{11}{32}$ of Dysprosium-Rim $E_{66}=\left[E_{1}+\frac{E 1}{4}+\frac{E 1}{9}+\frac{E 1}{16}+\frac{E 1}{25}\right]+\left(\frac{E 1}{36} \frac{11}{32}\right)+\left[\frac{E 1}{6^{2}} \frac{1}{2.4^{2}}\right]=\left[E_{1}+\frac{E 1}{4}+\frac{E 1}{9}+\frac{E 1}{16}+\frac{E 1}{25}\right]+\left(\frac{E 1}{36}\right) \cdot \frac{12}{32}$ of Holmium-Rim $\quad E_{67}=\left[E_{1}+\frac{E 1}{4}+\frac{E 1}{9}+\frac{E 1}{16}+\frac{E 1}{25}\right]+\left(\frac{E 1}{36} \frac{12}{32}\right)+\left[\frac{E 1}{6^{2}} \frac{1}{2.4}\right]=\left[E_{1}+\frac{E 1}{4}+\frac{E 1}{9}+\frac{E 1}{16}+\frac{E 1}{25}\right]+\left(\frac{E 1}{36}\right) \cdot \frac{13}{32}$ of Erbium-Rim $\quad E_{68}=\left[E_{1}+\frac{E 1}{4}+\frac{E 1}{9}+\frac{E 1}{16}+\frac{E 1}{25}\right]+\left(\frac{E 1}{36} \frac{13}{32}\right)+\left[\frac{E 1}{6^{2}} \frac{1}{2.4^{2}}\right]=\left[E_{1}+\frac{E 1}{4}+\frac{E 1}{9}+\frac{E 1}{16}+\frac{E 1}{25}\right]+\left(\frac{E 1}{36}\right) \cdot \frac{14}{32}$ of Thulium-Rim $\quad E_{69}=\left[E_{1}+\frac{E 1}{4}+\frac{E 1}{9}+\frac{E 1}{16}+\frac{E 1}{25}\right]+\left(\frac{E 1}{36} \frac{14}{32}\right)+\left[\frac{E 1}{6^{2}} \frac{1}{2 \cdot 4^{2}}\right]=\left[E_{1}+\frac{E 1}{4}+\frac{E 1}{9}+\frac{E 1}{16}+\frac{E 1}{25}\right]+\left(\frac{E 1}{36}\right) \cdot \frac{15}{32}$ of Ytterbium-Rim $\quad E_{70}=\left[E_{1}+\frac{E 1}{4}+\frac{E 1}{9}+\frac{E 1}{16}+\frac{E 1}{25}\right]+\left(\frac{E 1}{36} \frac{15}{32}\right)+\left[\frac{E 1}{6^{2}} \frac{1}{2.4^{2}}\right]=\left[E_{1}+\frac{E 1}{4}+\frac{E 1}{9}+\frac{E 1}{16}+\frac{E 1}{25}\right]+\left(\frac{E 1}{36}\right) \cdot \frac{16}{32}$ of Lutetium-Rim $\quad E_{71}=\left[E_{1}+\frac{E 1}{4}+\frac{E 1}{9}+\frac{E 1}{16}+\frac{E 1}{25}\right]+\left(\frac{E 1}{36} \frac{16}{32}\right)+\left[\frac{E 1}{6^{2}} \frac{1}{2.4^{2}}\right]=\left[E_{1}+\frac{E 1}{4}+\frac{E 1}{9}+\frac{E 1}{16}+\frac{E 1}{25}\right]+\left(\frac{E 1}{36}\right) \cdot \frac{17}{32}$ of Hafnium-Rim $\quad E_{72}=\left[E_{1}+\frac{E 1}{4}+\frac{E 1}{9}+\frac{E 1}{16}+\frac{E 1}{25}\right]+\left(\frac{E 1}{36} \frac{17}{32}\right)+\left[\frac{E 1}{6^{2}} \frac{1}{2.4^{2}}\right]=\left[E_{1}+\frac{E 1}{4}+\frac{E 1}{9}+\frac{E 1}{16}+\frac{E 1}{25}\right]+\left(\frac{E 1}{36}\right) \cdot \frac{18}{32}$ of Tantalum-Rim $\quad E_{73}=\left[E_{1}+\frac{E 1}{4}+\frac{E 1}{9}+\frac{E 1}{16}+\frac{E 1}{25}\right]+\left(\frac{E 1}{36} \frac{18}{32}\right)+\left[\frac{E 1}{6^{2}} \frac{1}{2.4^{2}}\right]=\left[E_{1}+\frac{E 1}{4}+\frac{E 1}{9}+\frac{E 1}{16}+\frac{E 1}{25}\right]+\left(\frac{E 1}{36}\right) \cdot \frac{19}{32}$ of Tungsten-Rim $\quad E_{74}=\left[E_{1}+\frac{E 1}{4}+\frac{E 1}{9}+\frac{E 1}{16}+\frac{E 1}{25}\right]+\left(\frac{E 1}{36} \frac{19}{32}\right)+\left[\frac{E 1}{6^{2}} \frac{1}{2.4^{2}}\right]=\left[E_{1}+\frac{E 1}{4}+\frac{E 1}{9}+\frac{E 1}{16}+\frac{E 1}{25}\right]+\left(\frac{E 1}{36}\right) \cdot \frac{20}{32}$ of Rhenium-Rim $\quad E_{75}=\left[E_{1}+\frac{E 1}{4}+\frac{E 1}{9}+\frac{E 1}{16}+\frac{E 1}{25}\right]+\left(\frac{E 1}{36} \frac{20}{32}\right)+\left[\frac{E 1}{6^{2}} \frac{1}{2 \cdot 4^{2}}\right]=\left[E_{1}+\frac{E 1}{4}+\frac{E 1}{9}+\frac{E 1}{16}+\frac{E 1}{25}\right]+\left(\frac{E 1}{36}\right) \cdot \frac{21}{32}$ of Osmium-Rim $\quad E_{76}=\left[E_{1}+\frac{E 1}{4}+\frac{E 1}{9}+\frac{E 1}{16}+\frac{E 1}{25}\right]+\left(\frac{E 1}{36} \frac{21}{32}\right)+\left[\frac{E 1}{6^{2}} \frac{1}{2 \cdot 4^{2}}\right]=\left[E_{1}+\frac{E 1}{4}+\frac{E 1}{9}+\frac{E 1}{16}+\frac{E 1}{25}\right]+\left(\frac{E 1}{36}\right) \cdot \frac{22}{32}$ of Iridium-Rim $\mathrm{E}_{77}=\left[\mathrm{E}_{1}+\frac{\mathrm{E} 1}{4}+\frac{\mathrm{E} 1}{9}+\frac{\mathrm{E} 1}{16}+\frac{\mathrm{E} 1}{25}\right]+\left(\frac{\mathrm{E} 1}{36} \frac{22}{32}\right)+\left[\frac{\mathrm{E} 1}{6^{2}} \frac{1}{2.4^{2}}\right]=\left[\mathrm{E}_{1}+\frac{\mathrm{E} 1}{4}+\frac{\mathrm{E} 1}{9}+\frac{\mathrm{E} 1}{16}+\frac{\mathrm{E} 1}{25}\right]+\left(\frac{\mathrm{E} 1}{36}\right) \cdot \frac{23}{32}$ of Platinum-Rim $\quad E_{78}=\left[E_{1}+\frac{E 1}{4}+\frac{E 1}{9}+\frac{E 1}{16}+\frac{E 1}{25}\right]+\left(\frac{E 1}{36} \frac{23}{32}\right)+\left[\frac{E 1}{6^{2}} \frac{1}{2.4^{2}}\right]=\left[E_{1}+\frac{E 1}{4}+\frac{E 1}{9}+\frac{E 1}{16}+\frac{E 1}{25}\right]+\left(\frac{E 1}{36}\right) \cdot \frac{24}{32}$ of Gold-Rim $E_{79}=\left[E_{1}+\frac{E 1}{4}+\frac{E 1}{9}+\frac{E 1}{16}+\frac{E 1}{25}\right]+\left(\frac{E 1}{36} \frac{24}{32}\right)+\left[\frac{E 1}{6^{2}} \frac{1}{2.4^{2}}\right]=\left[E_{1}+\frac{E 1}{4}+\frac{E 1}{9}+\frac{E 1}{16}+\frac{E 1}{25}\right]+\left(\frac{E 1}{36}\right) \cdot \frac{25}{32}$ of Mercury-Rim $\mathrm{E}_{80}=\left[\mathrm{E}_{1}+\frac{\mathrm{E} 1}{4}+\frac{\mathrm{E} 1}{9}+\frac{\mathrm{E} 1}{16}+\frac{\mathrm{E} 1}{25}\right]+\left(\frac{\mathrm{E} 1}{36} \frac{25}{32}\right)+\left[\frac{\mathrm{E} 1}{6^{2}} \frac{1}{2.4^{2}}\right]=\left[\mathrm{E}_{1}+\frac{\mathrm{E} 1}{4}+\frac{\mathrm{E} 1}{9}+\frac{\mathrm{E} 1}{16}+\frac{\mathrm{E} 1}{25}\right]+\left(\frac{\mathrm{E} 1}{36}\right) \cdot \frac{26}{32}$ of Thallium-Rim $E_{81}=\left[E_{1}+\frac{E 1}{4}+\frac{E 1}{9}+\frac{E 1}{16}+\frac{E 1}{25}\right]+\left(\frac{E 1}{36} \frac{26}{32}\right)+\left[\frac{E 1}{6^{2}} \frac{1}{2 \cdot 4^{2}}\right]=\left[E_{1}+\frac{E 1}{4}+\frac{E 1}{9}+\frac{E 1}{16}+\frac{E 1}{25}\right]+\left(\frac{E 1}{36}\right) \cdot \frac{27}{32}$ of Lead-Rim $\mathrm{E}_{82}=\left[\mathrm{E}_{1}+\frac{\mathrm{E} 1}{4}+\frac{\mathrm{E} 1}{9}+\frac{\mathrm{E} 1}{16}+\frac{\mathrm{E} 1}{25}\right]+\left(\frac{\mathrm{E} 1}{36} \frac{27}{32}\right)+\left[\frac{\mathrm{E} 1}{6^{2}} \frac{1}{2.4^{2}}\right]=\left[\mathrm{E}_{1}+\frac{\mathrm{E} 1}{4}+\frac{\mathrm{E} 1}{9}+\frac{\mathrm{E} 1}{16}+\frac{\mathrm{E} 1}{25}\right]+\left(\frac{\mathrm{E} 1}{36}\right) \cdot \frac{28}{32}$

of Bismuth-Rim

$$
\mathrm{E}_{83}=\left[\mathrm{E}_{1}+\frac{\mathrm{E} 1}{4}+\frac{\mathrm{E} 1}{9}+\frac{\mathrm{E} 1}{16}+\frac{\mathrm{E} 1}{25}\right]+\left(\frac{\mathrm{E} 1}{36} \frac{28}{32}\right)+\left[\frac{\mathrm{E} 1}{6^{2}} \frac{1}{2 \cdot 4^{2}}\right]=\left[\mathrm{E}_{1}+\frac{\mathrm{E} 1}{4}+\frac{\mathrm{E} 1}{9}+\frac{\mathrm{E} 1}{16}+\frac{\mathrm{E} 1}{25}\right]+\left(\frac{\mathrm{E} 1}{36}\right) \cdot \frac{29}{32}
$$

of Polonium-Rim $\quad E_{84}=\left[E_{1}+\frac{E 1}{4}+\frac{E 1}{9}+\frac{E 1}{16}+\frac{E 1}{25}\right]+\left(\frac{E 1}{36} \frac{29}{32}\right)+\left[\frac{E 1}{6^{2}} \frac{1}{2.4^{2}}\right]=\left[E_{1}+\frac{E 1}{4}+\frac{E 1}{9}+\frac{E 1}{16}+\frac{E 1}{25}\right]+\left(\frac{E 1}{36}\right) \cdot \frac{30}{32}$ of Astatine-Rim.6

$$
E_{85}=\left[E_{1}+\frac{E 1}{4}+\frac{E 1}{9}+\frac{E 1}{16}+\frac{E 1}{25}\right]+\left(\frac{E 1}{36} \frac{30}{32}\right)+\left[\frac{E 1}{6^{2}} \frac{1}{2.4^{2}}\right]=\left[E_{1}+\frac{E 1}{4}+\frac{E 1}{9}+\frac{E 1}{16}+\frac{E 1}{25}\right]+\left(\frac{E 1}{36}\right) \cdot \frac{31}{32}
$$

Radon W-Rim.6 $E_{\mathbf{8 6}}=\left[E_{1}+\frac{E 1}{4}+\frac{E 1}{9}+\frac{E 1}{16} \frac{E 1}{25}\right]+\left(\frac{E 1}{36} \frac{31}{32}\right)+\left[\frac{E 1}{6^{2}} \frac{1}{24^{2}}\right]=\left[E_{1}+\frac{E 1}{4}+\frac{E 1}{9}+\frac{E 1}{16}+\frac{E 1}{25}\right]+\frac{E 1}{36} \frac{32}{32}=\left[E_{1}+\frac{E 1}{4}+\frac{E 1}{9}+\frac{E 1}{16}+\frac{E 1}{25}+\frac{E 1}{36}\right]$

of Francium-Rim.7 $\mathrm{E}_{87}=\left[\mathrm{E}_{1}+\frac{\mathrm{E} 1}{4}+\frac{\mathrm{E} 1}{9}+\frac{\mathrm{E} 1}{16}+\frac{\mathrm{E} 1}{25}+\frac{\mathrm{E} 1}{36}\right]+\left[\frac{\mathrm{E} 1}{7^{2}} \frac{1}{2.4^{2}}\right]=\left[\mathrm{E}_{1}+\frac{\mathrm{E} 1}{4}+\frac{\mathrm{E} 1}{9}+\frac{\mathrm{E} 1}{16}+\frac{\mathrm{E} 1}{25}+\frac{\mathrm{E} 1}{36}\right]+\left(\frac{\mathrm{E} 1}{49}\right) \frac{1}{32}$ of Radium-Rim $\quad \mathrm{E}_{88}=\left[\mathrm{E}_{1}+\frac{\mathrm{E} 1}{4}+\frac{\mathrm{E} 1}{9}+\frac{\mathrm{E} 1}{16}+\frac{\mathrm{E} 1}{25}+\frac{\mathrm{E} 1}{36}\right]+\left(\frac{\mathrm{E} 1}{49}\right) \frac{1}{32}+\left[\frac{\mathrm{E} 1}{7^{2}} \frac{1}{2.4^{2}}\right]=\left[\mathrm{E}_{1}+\frac{\mathrm{E} 1}{4}+\frac{\mathrm{E} 1}{9}+\frac{\mathrm{E} 1}{16}+\frac{\mathrm{E} 1}{25}+\frac{\mathrm{E} 1}{36}\right]+\left(\frac{\mathrm{E} 1}{49}\right) \frac{2}{32}$ of Actinium-Rim $\quad E_{89}=\left[E_{1}+\frac{E 1}{4}+\frac{E 1}{9}+\frac{E 1}{16}+\frac{E 1}{25}+\frac{E 1}{36}\right]+\left(\frac{E 1}{49}\right) \frac{2}{32}+\left[\frac{E 1}{7^{2}} \frac{1}{2.4^{2}}\right]=\left[E_{1}+\frac{E 1}{4}+\frac{E 1}{9}+\frac{E 1}{16}+\frac{E 1}{25}++\frac{E 1}{36}\right]+\left(\frac{E 1}{49}\right) \frac{3}{32}$ 
of Thorium-Rim $\quad E_{90}=\left[E_{1}+\frac{E 1}{4}+\frac{E 1}{9}+\frac{E 1}{16}+\frac{E 1}{25}+\frac{E 1}{36}\right]+\left(\frac{E 1}{49}\right) \frac{3}{32}+\left[\frac{E 1}{7^{2}} \frac{1}{2.4^{2}}\right]=\left[E_{1}+\frac{E 1}{4}+\frac{E 1}{9}+\frac{E 1}{16}+\frac{E 1}{25}+\frac{E 1}{36}\right]+\left(\frac{E 1}{49}\right) \frac{4}{32}$ of Protactinium-Rim $E_{91}=\left[E_{1}+\frac{E 1}{4}+\frac{E 1}{9}+\frac{E 1}{16}+\frac{E 1}{25}+\frac{E 1}{36}\right]+\left(\frac{E 1}{49}\right) \frac{4}{32}+\left[\frac{E 1}{7^{2}} \frac{1}{2.4^{2}}\right]=\left[E_{1}+\frac{E 1}{4}+\frac{E 1}{9}+\frac{E 1}{16}+\frac{E 1}{25}+\frac{E 1}{36}\right]+\left(\frac{E 1}{49}\right) \frac{5}{32}$ of Uranium-Rim $\quad E_{92}=\left[E_{1}+\frac{E 1}{4}+\frac{E 1}{9}+\frac{E 1}{16}+\frac{E 1}{25}+\frac{E 1}{36}\right]+\left(\frac{E 1}{49}\right) \frac{5}{32}+\left[\frac{E 1}{7^{2}} \frac{1}{2.4^{2}}\right]=\left[E_{1}+\frac{E 1}{4}+\frac{E 1}{9}+\frac{E 1}{16}+\frac{E 1}{25}+\frac{E 1}{36}\right]+\left(\frac{E 1}{49}\right) \frac{6}{32}$ of Neptunium-Rim $E_{93}=\left[E_{1}+\frac{E 1}{4}+\frac{E 1}{9}+\frac{E 1}{16}+\frac{E 1}{25}+\frac{E 1}{36}\right]+\left(\frac{E 1}{49}\right) \frac{6}{32}+\left[\frac{E 1}{7^{2}} \frac{1}{2.4^{2}}\right]=\left[E_{1}+\frac{E 1}{4}+\frac{E 1}{9}+\frac{E 1}{16}+\frac{E 1}{25}+\frac{E 1}{36}\right]+\left(\frac{E 1}{49}\right) \frac{7}{32}$ of Plutonium-Rim $E_{94}=\left[E_{1}+\frac{E 1}{4}+\frac{E 1}{9}+\frac{E 1}{16}+\frac{E 1}{25}+\frac{E 1}{36}\right]+\left(\frac{E 1}{49}\right) \frac{7}{32}+\left[\frac{E 1}{7^{2}} \frac{1}{2.4^{2}}\right]=\left[E_{1}+\frac{E 1}{4}+\frac{E 1}{9}+\frac{E 1}{16}+\frac{E 1}{25}+\frac{E 1}{36}\right]+\left(\frac{E 1}{49}\right) \frac{8}{32}$ of Americium-Rim $\mathrm{E}_{95}=\left[\mathrm{E}_{1}+\frac{\mathrm{E} 1}{4}+\frac{\mathrm{E} 1}{9}+\frac{\mathrm{E} 1}{16}+\frac{\mathrm{E} 1}{25}+\frac{\mathrm{E} 1}{36}\right]+\left(\frac{\mathrm{E} 1}{49}\right) \frac{8}{32}+\left[\frac{\mathrm{E} 1}{7^{2}} \frac{1}{2.4^{2}}\right]=\left[\mathrm{E}_{1}+\frac{\mathrm{E} 1}{4}+\frac{\mathrm{E} 1}{9}+\frac{\mathrm{E} 1}{16}+\frac{\mathrm{E} 1}{25}+\frac{\mathrm{E} 1}{36}\right]+\left(\frac{\mathrm{E} 1}{49}\right) \frac{9}{32}$ of Curium-Rim $\quad E_{96}=\left[E_{1}+\frac{E 1}{4}+\frac{E 1}{9}+\frac{E 1}{16}+\frac{E 1}{25}+\frac{E 1}{36}\right]+\left(\frac{E 1}{49}\right) \frac{9}{32}+\left[\frac{E 1}{7^{2}} \frac{1}{2.4^{2}}\right]=\left[E_{1}+\frac{E 1}{4}+\frac{E 1}{9}+\frac{E 1}{16}+\frac{E 1}{25}+\frac{E 1}{36}\right]+\left(\frac{E 1}{49}\right) \frac{10}{32}$ of Berkelium-Rim $\mathrm{E}_{97}=\left[\mathrm{E}_{1}+\frac{\mathrm{E} 1}{4}+\frac{\mathrm{E} 1}{9}+\frac{\mathrm{E} 1}{16}+\frac{\mathrm{E} 1}{25}+\frac{\mathrm{E} 1}{36}\right]+\left(\frac{\mathrm{E} 1}{49}\right) \frac{10}{32}+\left[\frac{\mathrm{E} 1}{7^{2}} \frac{1}{2.4^{2}}\right]=\left[\mathrm{E}_{1}+\frac{\mathrm{E} 1}{4}+\frac{\mathrm{E} 1}{9}+\frac{\mathrm{E} 1}{16}+\frac{\mathrm{E} 1}{25}+\frac{\mathrm{E} 1}{36}\right]+\left(\frac{\mathrm{E} 1}{49}\right) \frac{11}{32}$ of Californium-Rim $E_{98}=\left[E_{1}+\frac{E 1}{4}+\frac{E 1}{9}+\frac{E 1}{16}+\frac{E 1}{25}+\frac{E 1}{36}\right]+\left(\frac{E 1}{49}\right) \frac{11}{32}+\left[\frac{E 1}{7^{2}} \frac{1}{2.4^{2}}\right]=\left[E_{1}+\frac{E 1}{4}+\frac{E 1}{9}+\frac{E 1}{16}+\frac{E 1}{25}+\frac{E 1}{36}\right]+\left(\frac{E 1}{49}\right) \frac{12}{32}$ of Einsteinium-Rim $E_{99}=\left[E_{1}+\frac{E 1}{4}+\frac{E 1}{9}+\frac{E 1}{16}+\frac{E 1}{25}+\frac{E 1}{36}\right]+\left(\frac{E 1}{49}\right) \frac{12}{32}+\left[\frac{E 1}{7^{2}} \frac{1}{2.4^{2}}\right]=\left[E_{1}+\frac{E 1}{4}+\frac{E 1}{9}+\frac{E 1}{16}+\frac{E 1}{25}+\frac{E 1}{36}\right]+\left(\frac{E 1}{49}\right) \frac{13}{32}$ of Fermium-Rim $\quad E_{100}=\left[E_{1}+\frac{E 1}{4}+\frac{E 1}{9}+\frac{E 1}{16}+\frac{E 1}{25}+\frac{E 1}{36}\right]+\left(\frac{E 1}{49}\right) \frac{13}{32}+\left[\frac{E 1}{7^{2}} \frac{1}{2.4^{2}}\right]=\left[E_{1}+\frac{E 1}{4}+\frac{E 1}{9}+\frac{E 1}{16}+\frac{E 1}{25}+\frac{E 1}{36}\right]+\left(\frac{E 1}{49}\right) \frac{14}{32}$ of Mendelevium-Rim $E_{101}=\left[E_{1}+\frac{E 1}{4}+\frac{E 1}{9}+\frac{E 1}{16}+\frac{E 1}{25}+\frac{E 1}{36}\right]+\left(\frac{E 1}{49}\right) \frac{14}{32}+\left[\frac{E 1}{7^{2}} \frac{1}{2.4^{2}}\right]=\left[E_{1}+\frac{E 1}{4}+\frac{E 1}{9}+\frac{E 1}{16}+\frac{E 1}{25}+\frac{E 1}{36}\right]+\left(\frac{E 1}{49}\right) \frac{15}{32}$ of Nobelium-Rim $\quad E_{102}=\left[E_{1}+\frac{E 1}{4}+\frac{E 1}{9}+\frac{E 1}{16}+\frac{E 1}{25}+\frac{E 1}{36}\right]+\left(\frac{E 1}{49}\right) \frac{15}{32}+\left[\frac{E 1}{7^{2}} \frac{1}{2.4^{2}}\right]=\left[E_{1}+\frac{E 1}{4}+\frac{E 1}{9}+\frac{E 1}{16}+\frac{E 1}{25}+\frac{E 1}{36}\right]+\left(\frac{E 1}{49}\right) \frac{16}{32}$ of Lawrencium-Rim $E_{103}=\left[E_{1}+\frac{E 1}{4}+\frac{E 1}{9}+\frac{E 1}{16}+\frac{E 1}{25}+\frac{E 1}{36}\right]+\left(\frac{E 1}{49}\right) \frac{16}{32}+\left[\frac{E 1}{7^{2}} \frac{1}{2.4^{2}}\right]=\left[E_{1}+\frac{E 1}{4}+\frac{E 1}{9}+\frac{E 1}{16}+\frac{E 1}{25}+\frac{E 1}{36}\right]+\left(\frac{E 1}{49}\right) \frac{17}{32}$ of Rutherfordium-Rim $\mathrm{E}_{104}=\left[\mathrm{E}_{1}+\frac{\mathrm{E} 1}{4}+\frac{\mathrm{E} 1}{9}+\frac{\mathrm{E} 1}{16}+\frac{\mathrm{E} 1}{25}+\frac{\mathrm{E} 1}{36}\right]+\left(\frac{\mathrm{E} 1}{49}\right) \frac{17}{32}+\left[\frac{\mathrm{E} 1}{7^{2}} \frac{1}{2.4^{2}}\right]=\left[\mathrm{E}_{1}+\frac{\mathrm{E} 1}{4}+\frac{\mathrm{E} 1}{9}+\frac{\mathrm{E} 1}{16}+\frac{\mathrm{E} 1}{25}+\frac{\mathrm{E} 1}{36}\right]+\left(\frac{\mathrm{E} 1}{49}\right) \frac{18}{32}$ of Dubnium-Rim $\quad E_{105}=\left[E_{1}+\frac{E 1}{4}+\frac{E 1}{9}+\frac{E 1}{16}+\frac{E 1}{25}+\frac{E 1}{36}\right]+\left(\frac{E 1}{49}\right) \frac{18}{32}+\left[\frac{E 1}{7^{2}} \frac{1}{2.4^{2}}\right]=\left[E_{1}+\frac{E 1}{4}+\frac{E 1}{9}+\frac{E 1}{16}+\frac{E 1}{25}+\frac{E 1}{36}\right]+\left(\frac{E 1}{49}\right) \frac{19}{32}$ of Seaborgium-Rim $E_{106}=\left[E_{1}+\frac{E 1}{4}+\frac{E 1}{9}+\frac{E 1}{16}+\frac{E 1}{25}+\frac{E 1}{36}\right]+\left(\frac{E 1}{49}\right) \frac{19}{32}+\left[\frac{E 1}{7^{2}} \frac{1}{2.4^{2}}\right]=\left[E_{1}+\frac{E 1}{4}+\frac{E 1}{9}+\frac{E 1}{16}+\frac{E 1}{25}+\frac{E 1}{36}\right]+\left(\frac{E 1}{49}\right) \frac{20}{32}$ of Bohrium-Rim $\quad E_{107}=\left[E_{1}+\frac{E 1}{4}+\frac{E 1}{9}+\frac{E 1}{16}+\frac{E 1}{25}+\frac{E 1}{36}\right]+\left(\frac{E 1}{49}\right) \frac{20}{32}+\left[\left[\frac{E 1}{7^{2}} \frac{1}{2.4^{2}}\right]=\left[E_{1}+\frac{E 1}{4}+\frac{E 1}{9}+\frac{E 1}{16}+\frac{E 1}{25}+\frac{E 1}{36}\right]+\left(\frac{E 1}{49}\right) \frac{21}{32}\right.$ of Hassium-Rim $\quad E_{108}=\left[E_{1}+\frac{E 1}{4}+\frac{E 1}{9}+\frac{E 1}{16}+\frac{E 1}{25}+\frac{E 1}{36}\right]+\left(\frac{E 1}{49}\right) \frac{21}{32}+\left[\frac{E 1}{7^{2}} \frac{1}{2.4^{2}}\right]=\left[E_{1}+\frac{E 1}{4}+\frac{E 1}{9}+\frac{E 1}{16}+\frac{E 1}{25}+\frac{E 1}{36}\right]+\left(\frac{E 1}{49}\right) \frac{22}{32}$ of Meitrenium-Rim $E_{109}=\left[E_{1}+\frac{E 1}{4}+\frac{E 1}{9}+\frac{E 1}{16}+\frac{E 1}{25}+\frac{E 1}{36}\right]+\left(\frac{E 1}{49}\right) \frac{22}{32}+\left[\frac{E 1}{7^{2}} \frac{1}{2.4^{2}}\right]=\left[E_{1}+\frac{E 1}{4}+\frac{E 1}{9}+\frac{E 1}{16}+\frac{E 1}{25}+\frac{E 1}{36}\right]+\left(\frac{E 1}{49}\right) \frac{23}{32}$ of Darmstadum-Rim $E_{110}=\left[E_{1}+\frac{E 1}{4}+\frac{E 1}{9}+\frac{E 1}{16}+\frac{E 1}{25}+\frac{E 1}{36}\right]+\left(\frac{E 1}{49}\right) \frac{23}{32}+\left[\frac{E 1}{7^{2}} \frac{1}{2.4^{2}}\right]=\left[E_{1}+\frac{E 1}{4}+\frac{E 1}{9}+\frac{E 1}{16}+\frac{E 1}{25}+\frac{E 1}{36}\right]+\left(\frac{E 1}{49}\right) \frac{24}{32}$ of Roentgenium-Rim $E_{111}=\left[E_{1}+\frac{E 1}{4}+\frac{E 1}{9}+\frac{E 1}{16}+\frac{E 1}{25}+\frac{E 1}{36}\right]+\left(\frac{E 1}{49}\right) \frac{24}{32}+\left[\frac{E 1}{7^{2}} \frac{1}{2.4^{2}}\right]=\left[E_{1}+\frac{E 1}{4}+\frac{E 1}{9}+\frac{E 1}{16}+\frac{E 1}{25}+\frac{E 1}{36}\right]+\left(\frac{E 1}{49}\right) \frac{25}{32}$ of Copernicium-Rim $E_{112}=\left[E_{1}+\frac{E 1}{4}+\frac{E 1}{9}+\frac{E 1}{16}+\frac{E 1}{25}+\frac{E 1}{36}\right]+\left(\frac{E 1}{49}\right) \frac{25}{32}+\left[\frac{E 1}{7^{2}} \frac{1}{2.4^{2}}\right]=\left[E_{1}+\frac{E 1}{4}+\frac{E 1}{9}+\frac{E 1}{16}+\frac{E 1}{25}+\frac{E 1}{36}\right]+\left(\frac{E 1}{49}\right) \frac{26}{32}$ of Unutrium-Rim $\quad E_{113}=\left[E_{1}+\frac{E 1}{4}+\frac{E 1}{9}+\frac{E 1}{16}+\frac{E 1}{25}+\frac{E 1}{36}\right]+\left(\frac{E 1}{49}\right) \frac{26}{32}+\left[\frac{E 1}{7^{2}} \frac{1}{2.4^{2}}\right]=\left[E_{1}+\frac{E 1}{4}+\frac{E 1}{9}+\frac{E 1}{16}+\frac{E 1}{25}+\frac{E 1}{36}\right]+\left(\frac{E 1}{49}\right) \frac{27}{32}$ of Flerovium-Rim $E_{114}=\left[E_{1}+\frac{E 1}{4}+\frac{E 1}{9}+\frac{E 1}{16}+\frac{E 1}{25}+\frac{E 1}{36}\right]+\left(\frac{E 1}{49}\right) \frac{27}{32}+\left[\frac{E 1}{7^{2}} \frac{1}{2.4^{2}}\right]=\left[E_{1}+\frac{E 1}{4}+\frac{E 1}{9}+\frac{E 1}{16}+\frac{E 1}{25}+\frac{E 1}{36}\right]+\left(\frac{E 1}{49}\right) \frac{28}{32}$ of Ununpentium-Rim $E_{115}=\left[E_{1}+\frac{E 1}{4}+\frac{E 1}{9}+\frac{E 1}{16}+\frac{E 1}{25}+\frac{E 1}{36}\right]+\left(\frac{E 1}{49}\right) \frac{28}{32}+\left[\frac{E 1}{7^{2}} \frac{1}{2.4^{2}}\right]=\left[E_{1}+\frac{E 1}{4}+\frac{E 1}{9}+\frac{E 1}{16}+\frac{E 1}{25}+\frac{E 1}{36}\right]+\left(\frac{E 1}{49}\right) \frac{29}{32}$ of Livermorium-Rim $E_{116}=\left[E_{1}+\frac{E 1}{4}+\frac{E 1}{9}+\frac{E 1}{16}+\frac{E 1}{25}+\frac{E 1}{36}\right]+\left(\frac{E 1}{49}\right) \frac{29}{32}+\left[\frac{E 1}{7^{2}} \frac{1}{2.4^{2}}\right]=\left[E_{1}+\frac{E 1}{4}+\frac{E 1}{9}+\frac{E 1}{16}+\frac{E 1}{25}+\frac{E 1}{36}\right]+\left(\frac{E 1}{49}\right) \frac{30}{32}$ of Ununseptium-S.Rim.7 $E_{117}=\left[E_{1}+\frac{E 1}{4}+\frac{E 1}{9}+\frac{E 1}{16}+\frac{E 1}{25}+\frac{E 1}{36}\right]+\left(\frac{E 1}{49}\right) \frac{30}{32}+\left[\frac{E 1}{7^{2}} \frac{1}{2.4^{2}}\right]=\left[E_{1}+\frac{E 1}{4}+\frac{E 1}{9}+\frac{E 1}{16}+\frac{E 1}{25}+\frac{E 1}{36}\right]+\left(\frac{E 1}{49}\right) \frac{31}{32}$ Uuo $E_{118}=\left[E_{1}+\frac{E 1}{4}+\frac{E 1}{9}+\frac{E 1}{16}+\frac{E 1}{25}+\frac{E 1}{36}\right]+\left(\frac{E 1}{49}\right) \frac{31}{32}+\left[\frac{E 1}{7^{2}} \frac{1}{2.4^{2}}\right]=\left[E_{1}+\frac{E 1}{4}+\frac{E 1}{9}+\frac{E 1}{16}+\frac{E 1}{25}+\frac{E 1}{36}\right]+\left(\frac{E 1}{49}\right) \frac{32}{32}=\left[E_{1}+\frac{E 1}{4}+\frac{E 1}{9}+\frac{E 1}{16}+\frac{E 1}{25}+\frac{E 1}{36}+\frac{E 1}{49}\right]$ of Ma1-S.Rim. $8 \quad E_{119}=\left[E_{1}+\frac{E 1}{4}+\frac{E 1}{9}+\frac{E 1}{16}+\frac{E 1}{25}+\frac{E 1}{36}+\frac{E 1}{49}\right]+\left[\frac{E 1}{8^{2}} \frac{1}{2.5^{2}}\right]=\left[E_{1}+\frac{E 1}{4}+\frac{E 1}{9}+\frac{E 1}{16}+\frac{E 1}{25}+\frac{E 1}{36}+\frac{E 1}{49}\right]+\left[\frac{E 1}{64}\right] \cdot \frac{1}{50}$ 
of Ma2-Rim $E_{120}=\left[E_{1}+\frac{E 1}{4}+\frac{E 1}{9}+\frac{E 1}{16}+\frac{E 1}{25}+\frac{E 1}{36}+\frac{E 1}{49}\right]+\left[\frac{E 1}{64}\right] \cdot \frac{1}{50}+\left[\frac{E 1}{8^{2}} \frac{1}{2.5^{2}}\right]=\left[E_{1}+\frac{E 1}{4}+\frac{E 1}{9}+\frac{E 1}{16}+\frac{E 1}{25}+\frac{E 1}{36}+\frac{E 1}{49}\right]+\left[\frac{E 1}{64}\right] \cdot \frac{2}{50}$ of Ma3-Rim $E_{121}=\left[E_{1}+\frac{E 1}{4}+\frac{E 1}{9}+\frac{E 1}{16}+\frac{E 1}{25}+\frac{E 1}{36}+\frac{E 1}{49}\right]+\left[\frac{E 1}{64}\right] \cdot \frac{2}{50}+\left[\frac{E 1}{8^{2}} \frac{1}{2.5^{2}}\right]=\left[E_{1}+\frac{E 1}{4}+\frac{E 1}{9}+\frac{E 1}{16}+\frac{E 1}{25}+\frac{E 1}{36}+\frac{E 1}{49}\right]+\left[\frac{E 1}{64}\right] \cdot \frac{3}{50}$ of Ma4-Rim $E_{122}=\left[E_{1}+\frac{E 1}{4}+\frac{E 1}{9}+\frac{E 1}{16}+\frac{E 1}{25}+\frac{E 1}{36}+\frac{E 1}{49}\right]+\left[\frac{E 1}{64}\right] \cdot \frac{3}{50}+\left[\frac{E 1}{8^{2}} \frac{1}{2.5^{2}}\right]=\left[E_{1}+\frac{E 1}{4}+\frac{E 1}{9}+\frac{E 1}{16}+\frac{E 1}{25}+\frac{E 1}{36}+\frac{E 1}{49}\right]+\left[\frac{E 1}{64}\right] \cdot \frac{4}{50}$ of Ma5-Rim $E_{123}=\left[E_{1}+\frac{E 1}{4}+\frac{E 1}{9}+\frac{E 1}{16}+\frac{E 1}{25}+\frac{E 1}{36}+\frac{E 1}{49}\right]+\left[\frac{E 1}{64}\right] \cdot \frac{4}{50}+\left[\frac{E 1}{8^{2}} \frac{1}{2.5^{2}}\right]=\left[E_{1}+\frac{E 1}{4}+\frac{E 1}{9}+\frac{E 1}{16}+\frac{E 1}{25}+\frac{E 1}{36}+\frac{E 1}{49}\right]+\left[\frac{E 1}{64}\right] \cdot \frac{5}{50}$ of C W-Rim $\quad \mathrm{E}_{\mathrm{c}}=\mathrm{E}_{\mathrm{c}-1}+\left[\frac{\mathrm{E} 1}{\mathrm{Rc}^{2}} \frac{1}{2 \cdot \mathrm{Rc}^{2}}\right]$ where $\mathrm{W}-\mathrm{Rc}=1,2, \ldots \mathrm{c}$, Number of $\mathrm{W}$ $\operatorname{Rim}$.

Following above logic all Particles or Atoms are formulated in this Geometrical formula without any Assumptions, or Axioms, or Exclusion Principles, or any other Starting Point . Because S-Rims are shifted, the shape of Stereo-Slices is a Double Helix , as this happens to DNA structure and is dependent on the number of the Rims . All of DNA can be created from this Gyroscope-Stereo-Slice Mechanism -GSSM- . From above is seen that Electrons of an Atom occupy Quantum levels or orbitals starting from the lowest Energy-level and proceeding to the higher. The GSSM mechanism is Dynamically applied on Nucleus Protons and is composed of concentric spherical Wheel-Rims,A Pattern on the torus ring,with Total Spin perpendicularly placed on $\mathrm{X}-\mathrm{Y}$, The Rotor, plane following the complexity of Rims.

The Periodic Table is now easily drawn . markos 15 / 5 / 2016.

Rearranging Periodic-Table according to Energy-State then the Proposed Periodic-Table becomes.

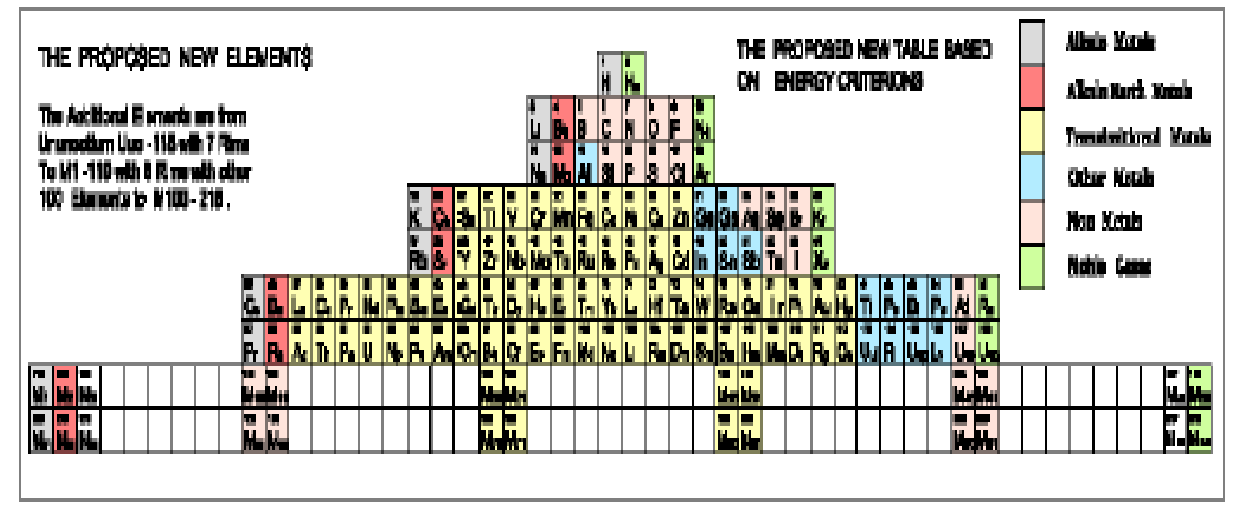

Figure 19.

The New Proposed Periodic Table of Atoms with the 218 , Protons , Elements .The Proposed New elements become from the completion of the Open-Rim (7) .

New Rim (8) is consisted of 50 available Positions which are filled with Electrons. The New is following Pascal`s-Triangle-Array where Rims are numbers contained in the Prior ones.

\subsection{The periodic Table of Elements .}

Since Material geometry follows Euclidean so and those of Spaces, Anti-Spaces and Sub-spaces. In [911] was shown that all Spaces coexist in the same Space and Sub-space. Since also Monads are Quaternion then rules are strictly applied. By Applying this logic in Material Moulds then In-between infinite Spaces and points must be contained in the next as this happens in the series of integers i.e. The number of places of Electrons inbetween $\mathrm{ON}-1 \mathrm{~N}-$ Orbitals must be $2 \cdot \mathrm{N}^{2}-2(\mathrm{~N}-1)^{2}=2 \cdot(2 \mathrm{~N}-1)$, and for $\mathrm{N}=6$ then then $\mathrm{N} 56=22$.

Each Element [ E] Contains the Prior [ E-1] Plus SLU = The Allowed by the Space Units . SLU $=$ Shell-Least-Unit, is $\rightarrow$ Full of Outer Permissive Sites and with Low Reactivity .

\section{Conclusions}

Any moving monad $[\mathrm{z}=\mathrm{s}+\mathrm{i} \cdot \overline{\mathrm{v}}]=$ as Work is, is transformed ( quantized ) into $\rightarrow$

1.In Elastic material Configuration, as Strain Energy and is absorbed as Support Reactions and displacement field $[\square \varepsilon(\overline{\mathrm{u}}, \overline{\mathrm{v}}, \overline{\mathrm{w}})]$ upon the deformed placement, (where these alterations of shape by pressure or stress is the equilibrium state of the Configuration [26], and then equations of Elasticity are , $\mathrm{G} . \square^{2} \varepsilon+[\mathrm{m} . \mathrm{G} /(\mathrm{m}-2)] . \square[\square \varepsilon]=\mathrm{F}$, or in isotropic material $\left.\rightarrow[\mu \square \mathcal{\varepsilon}+(\lambda+\mu) . \square(\square \varepsilon)]+\mathrm{F}=0\right]$. 
The intrinsic Stationary Electro-magnetic Wave of gravity, is binding as an dipole the two edge material points of this Homogenous- Isotropic, Rest and mass-less, and also of Elastic nature material Configuration Field . [22-23].

2.In Solid material Configuration, is as Kinetic (Energy of motion $\bar{v}$ ) and Potential (Stored Energy) energy by displacement (the magnitude of a vector from initial to subsequent position) and rotation, on the principal axis (through center of mass of the Solid ) as ellipsoid, which is mapped out , by the nib of vector $(\bar{\delta} . c)=[\bar{v} \cdot c+\bar{w} \cdot \bar{r} n] \delta t$, as the Inertia ellipsoid [Poinsot's ellipsoid construction ] in [S] frame which instantaneously rotates around vector axis $\overline{\mathrm{w}}, \varphi$ with the constant polar distance $\overline{\mathrm{W}} . \mathrm{Fe} /|\mathrm{Fe}|$ and the constant angles $\theta \mathrm{s}, \theta \mathrm{b}$, traced on, Reference [R] cone and on [S] cone, which are rolling around the common axis of $\overline{\mathrm{w}}$ vector without slipping, and if , Fe , is the Diagonal of the Energy Cuboids with dimensions a,b,c which follow Pythagoras conservation law, then the three magnitudes (J,E,B) of Energy -state follow Cuboids (Cycloid), Plane, or Linear Diagonal direction , and If Potential Energy is zero , then vector $\bar{w}$ is on the surface of the Inertia Ellipsoid . [23-27-28].

3. In Quaternion Extensive Configuration, as New Quaternion`s (with Scalar and Vector magnitudes ). Points in Primary Space [PNS] carry A -priori the work W $=\int \mathrm{A}-\mathrm{B}[\mathrm{P} . \mathrm{ds}]=0$, where magnitudes $\mathrm{P}$, $\overline{\mathrm{ds}}$ can be varied leaving work unaltered. The Diffusion (decomposition) of Energy is as the mechanism of Energy Transport as velocity $(\overline{\mathrm{v}})$, through its quantized wave-length $|\lambda|$, which is a property of any standing wave , into the Medium $|\lambda|=(1)-(2)$, and involves the Absorption and Reemission of the wave quantized energy $J=(J 1)=$ ( J2) as Electric and Magnetic field of Electric Displacement of the two neighbor edges (1) and (2) of the medium , following the isochrones Cycloidal motion . [37-39]

4.. In the Space conserved Extensive property Continuum ( the Spatial Configuration caves ), as Kinetic ( the 3-current motion ) and Potential (the perpendicular Stored curl fields) Energy by Cycloid motion ( the magnitude of wavelength vector $|\lambda|$ ), from the initial (1) to subsequent position (2), as it is the wavelength $|\lambda|=(1)-(2)$.

During shifting Energy as velocity vector $\quad, \bar{v}$, (and this because extreme case happens for zero application area ) is decomposed into two velocity vectors $\overline{\mathrm{v}} 1, \overline{\mathrm{v}} 2$, being vectors, undergo vibrations which causes two waves that represent the two Electric and Magnetic perpendicular, in Planes, components until reaching point (2) which is the Reemission of the wave and it is the new head of velocity, $\bar{v}$, where then mechanism is recycled .[39-40]

5.. The dynamics of any System = Work = Total Energy, is transferred as generalized force Qn as, Qn = $\partial \mathrm{W} / \partial(\bar{\delta} \overline{\mathrm{q}}),(\overline{\delta \mathrm{q}})=\overline{\mathrm{v}} \mathrm{n} \cdot \delta \mathrm{t}=[\overline{\mathrm{v}} \mathrm{c}+\overline{\mathrm{W}} \cdot \overline{\mathrm{r}} \mathrm{n}] \delta \mathrm{t}=($ Translational + Rotational velocity $) . \overline{\mathrm{t}} \mathrm{t}$ as velocity and Qn $=[\overline{\mathrm{v}} \mathrm{c}$. $(\partial \mathrm{T} / \delta \mathrm{t})+\overline{\mathrm{w}} \cdot \mathrm{rn}] .(\partial \mathrm{T} / \delta \mathrm{t}) \rightarrow$ Translational kinetic energy + Rotational kinetic energy as the Total Energy of the system . [40]

6.. The ultimate Constituents of Monads $(\mathrm{s}, \overline{\mathrm{v}} . \nabla \mathrm{i})$ is the real part , $|\mathrm{s}|$, and the Magnitude of Imaginary part as Vector $\overline{\mathrm{v}}=\nabla \mathrm{i}$, decomposed into velocity vectors $\overline{\mathrm{v}} 1, \overline{\mathrm{v}} 2$. [STPL] cylinder is a Geometrical Mechanism (Mould) which transfers the two Quantities of the breakable monads from one Level (Confinement) to another Level using Quantities or the Breakages of collision between monads .

This Mechanism is not the Origin of monads, but a Mould (the Regulative Universe Energy Valve) .

It was shown that into Gravity cave, $\mathrm{Lg}=2 . \mathrm{r}=e^{-i \cdot(-9 . \pi / 2) b)}=3,969.10^{-62} \mathrm{~m}$

is inversely balancing the Common circle, of Space, Anti-Space .

For the Rotational Spaces, with velocities $[\overline{\mathrm{v}} \mathrm{g}=\overline{\mathrm{w}} \mathrm{r}=\overline{\mathrm{c}}$ ] that of light, $\overline{\mathrm{c}}$, tending to zero in cave $\mathrm{Lc}>\mathrm{Lg}$ then exist velocities $[\bar{v} c>\bar{c}]$ tending to infinity. The hidden pattern of universe is, the STPL line, which is off the Spaces and connect them (it maintains, conserve and support all universe), so may say, it is The Navel Cord (string) of Galaxies) . [33-34]. i.e.

In Common Circle ( the Sub-Space ) of the rotating Space Anti-Space $[ \pm \Lambda]$, with maximum angular Velocity Vector, $\overline{\mathrm{v}}=\overline{\mathrm{w}} \cdot \mathrm{r}$ on circumference, [in the absence of applied Torques and because of the Birefringence property of stress continuum with different indices ,n, of refraction, which this creates the Retardation , $\delta$, and determining Color Bands or Fringes ] is Produced a color Spectrum which is, the $\langle$ Color Forces $\rangle \rightarrow$ Gluon Red ,Gluon Green, Gluon Blue. [36,41].

When the tangential velocity $\overline{\mathrm{v}}=\overline{\mathrm{w}}$.r on the circumference of a cave , r, is in another of radius $\mathrm{R}>\mathrm{r}$, then the new tangential velocity $\overline{\mathrm{v}}=\overline{\mathrm{w}} \cdot \mathrm{R}$ is greater than $\overline{\mathrm{v}}$ and when $\overline{\mathrm{v}}$ is the speed of light, then the new $\overline{\mathrm{v}}$, are velocities greater than that of light, and this is a way of succeeding these velocities .

7.In Black holes Energy scale $[|\lambda| . \Lambda=\mathrm{k} 1]$ there are infinite high frequency small amplitude vacuum fluctuations at Planck energy density of $10113 \mathrm{~J} / \mathrm{m} 3$ that exert action (pressure) on the moving Spaces dipole and their Stability is always achieved by Anti-space in their rotational equilibrium. [43]

8.Dipole vectors are quaternion`s (versors) of waving nature, i.e. , of one wavelength $|\lambda|$ in circumference in energy levels , that conserve energy by transferring the Total kinetic energy $\mathrm{T}$ into angular momentum $\mathrm{L}=\overline{\mathrm{r} m \bar{v}}=\overline{\mathrm{r}} \cdot \overline{\mathrm{p}}=\overline{\mathrm{r}} \Lambda$, as the Stationary Electromagnetic wave $[\mathbf{E} \perp \mathbf{H}]$ in constant mass $\mathrm{m}= \pm\left[(\overline{\mathrm{w}} \cdot \mathrm{r})^{2}\right]$ $=$ the reaction to this motion $\mathrm{v}^{2}=(\mathrm{wr})^{2}$. Different versors with different Energy (scalar) possess the same angular 
momentum . A Composition of Scalar Fields (s) and Vector Fields ( $\bar{v}$ ) of a frame to a new unit, maps the alterations of Unit by rotation only and transforms scalar magnitudes (particle properties) to vectors (wave properties) and vice-versa , and so , all particle-like properties are both of waves and particles. In Planck Scale, when the electron is being accelerated by gravity which exists in all energy levels as above, gravity is still exerting its force, so Electrodynamics can be derived from Newton's second law. [31-36] .

\subsection{The Geometrical Caves and}

The Energy-magnitudes . [29-41] All particles are characterized by their quantized cave inner the Space $\left[\mathrm{V}=4 \lambda^{2} / 2 \pi=4\left(\mathrm{w}^{2} \cdot \mathrm{r}^{2}\right)^{2} / 2 \pi\right]$ composed of an inner Electromagnetic wave constituent $[\mathrm{E} \square \mathrm{H}]$, and $\mathrm{h}=$ $2 \pi / \lambda$, is the reaction to these velocity motions [it is the mass $m=(\mathrm{pr}) \cdot \mathrm{w} / \mathrm{c}^{2}=\mathrm{h} \cdot \mathrm{w} / 2 \pi \mathrm{c}^{2}$ ], the external energy momentum is $\mathrm{p}=\mathrm{h} / 2 \mathrm{m \lambda}$ and Spin (the helically rotating electric field vector corresponding to a circularly polarized Electromagnetic wave and propagating with attenuation to the right). Conservation laws of energy linear and angular momentum are satisfied by the inner Anti-cycloidal motion, (lying on the evolute curve).

This interpretation of caves when applied to Young`s interference and Polarizer experiments is shown the unified understanding between the classical Electromagnetic field and the quantum particle of light.

This tiny energy volume for wave's case, is the Inner Cycloidal volume $V=4 / 3 \cdot \pi \cdot a b^{2}=4 \lambda^{3} / 3 \pi$,

[ because $\mathrm{a}=\lambda, \mathrm{b}=\lambda / \pi$ ] of the Electromagnetic stationary field $[\overline{\mathrm{E}} \mathrm{x} \overline{\mathrm{H}}]$ where Intensity $\mathrm{Sc}=\left[\varepsilon \cdot \mathrm{E}^{2} / 2+\mu \cdot \mathrm{H}^{2} / 2\right]$ and $\varepsilon, \mu$ the Permittivity, (it is the Dielectric constant multiplier), Permeability (the Dielectric constant multiplier).

Electromagnetic waves are able to transmit energy through a vacuum (empty space) by storing their energy in a, Dark, Standing Transverse Electromagnetic dipole wave as above, and thus considered completely particle like, and in transverse interference pattern to be considered as completely wave, so when Energy Intensity is,

a.Energy $I d=\rho \pi^{2} c^{3} 2 \lambda^{2}\left[\varepsilon E^{2}+\mu H^{2}\right]$ in volume $V=\left[V=4 \lambda^{2} / 2 \pi=4\left(w^{2} \cdot r^{2}\right)^{2} / 2 \pi\right] \rightarrow$ Energy behaves as Particle like

b.Energy Id $=\left(\right.$ p.c2). $(\text { wAo })^{2}$ in any Interference Pattern $\rightarrow$ Energy behaves as Wave like, Discrete

This is the Wave-Particle duality unifying the classical Electromagnetic field and the quantum particle of light.

\section{The Geometrical caves : [29]}

Nature has not any < meter > to measure quantized Quantities ( that of Space and of Energy) except these of the Geometry constants, one of which is number, $\pi$ (Archimedes number $\pi$ ) so quantization of Points $(\lambda)$ follows geometry constant $(\pi)$ and for Energy Wd, which is the quantized Energy of the Quantity dissipated per cycle , [ and this because monads follow sinusoidal oscillation on wavelength $=$ monads as the w.th power and the n.th root of this monad where w.n $=1$ as above on and in the same monad] and which energy is equal to ,

$\mathrm{Wd}=\int \dot{\mathrm{x}} \cdot \mathrm{dx}=\int \mathrm{C} \dot{\mathrm{x}}^{2} \cdot \mathrm{dt}=(\pi \mathrm{CW}) \cdot \lambda^{2} / 4=\left(2 \pi^{2} \mathrm{C} \cdot \lambda^{2} / 4\right) \cdot \mathrm{f}=\left(\mathrm{m} \pi \cdot \lambda^{2} / 2\right) . \mathrm{f}=\mathrm{C}$.f i.e. From above monads $(\mathrm{s}+\overline{\mathrm{v}} \square \mathrm{i})$ $1 / \mathrm{w}=|\mathrm{zo}|^{-} \mathrm{w} . \mathrm{e}^{\wedge}-\mathrm{i} \cdot(\varphi+2 \mathrm{k} \pi) . \mathrm{w}$, where $\cos \varphi=\mathrm{s} /|\mathrm{zo}|$, and for Rotated Energy case where $\mathrm{s}=0$ and also $\cos \cdot \varphi=0$ exists for angle $\varphi=\pi / 2$, the quarternion, $\mathrm{z}$, is $\mathrm{z}=(\mathrm{s}+\overline{\mathrm{V}} \nabla \mathrm{i})^{1 /} \mathrm{w}$ as dimension power $\mathrm{w}=\mathrm{b}$ and it is as $\mathrm{e}-\mathrm{i} \cdot(\pi 2+2 \mathrm{~km}) \cdot \mathrm{w}=\mathrm{e}-\mathrm{i} \cdot(5 \mathrm{\pi} 2) \cdot \mathrm{b}=\mathrm{e}-\mathrm{i} \cdot(5 \mathrm{\pi} 2) \cdot 10$ where, $\mathrm{Lp}=$ ei. $(-5 \pi 2) \cdot 10$ and it is the basic Geometrical interpretation of < Planck scale meter > based on the twoGeometry constants e, $\pi$ where $k=1$, and base $b=10$ ,and this from logarithm properties with different bases on the same base $\mathrm{e}$ [ when ey= $\mathrm{x}$ then base e logarithm of $\mathrm{x}$ is $\ln (\mathrm{x})=\operatorname{loge}(\square)=\mathrm{y}$ ] as it is ew $=(\mathrm{blog}(\mathrm{b}) \mathrm{e}) \mathrm{w}=\mathrm{bw} \cdot \log (\mathrm{b})$ eand $\sqrt{\mathrm{e}} \mathrm{w}=\mathrm{e} 1 / \mathrm{w}=\mathrm{e}-\mathrm{w}=\mathrm{x} 1 \mathrm{w} \cdot \log (\mathrm{b}) \mathrm{e}$ which are monads in monads and is of Wave motion with angular velocity $w=4 . \mathrm{Wd} /\left(\pi \cdot \mathrm{C} . \lambda^{2}\right)$, i.e. Space and Energy is quantized and measured on the two Constant and Natural numbers e , $\pi$, where for base the natural logarithm ,e, and exponent the decimal base, $\mathrm{b}=10$, where then issues $\rightarrow$ During Diffraction ,ds, frequency ,f, doesn't change and only the velocity,$\overline{\mathrm{v}}$, and wavelength,$\lambda$, changes and the Total Energy is $[\overline{\mathrm{z}} 1 / \mathrm{w}=|\overline{\mathrm{zo}}| \mathrm{w}$. Lo $]$. Again , because for quaternion, $\mathrm{z}$, exists $\rightarrow \mathrm{z}^{1 / 1} \mathrm{w}=(\mathrm{s}+\overline{\mathrm{v}} \nabla \mathrm{i}) 1 / \mathrm{w}=|\mathrm{zo}|^{-} \mathrm{w} \cdot[\cos \cdot(\varphi+\mathrm{kT}) / \mathrm{w}+\mathrm{i} \cdot \sin \cdot(\varphi+\mathrm{kT \pi}) / \mathrm{w}]=|\mathrm{zo}|^{-}$ w.e ${ }^{\wedge} \mathrm{i} .(\varphi+\mathrm{k} \pi) / \mathrm{w}$, and so then, For $\cos .(\varphi+\mathrm{k} \pi) / \mathrm{w}=0$ then exists only the Imaginary part of monad , $(\overline{\mathrm{v}} \nabla \mathrm{i}) \neq$ 0 , where $\varphi=\pi / 2$ and then $\mathrm{z}^{1 / \mathrm{w}}=|\mathrm{zo}|^{-} \mathrm{w}$. e i. $(\varphi+\mathrm{k} \pi) / \mathrm{w}= \pm \mathrm{i} .(\pi / 2+\mathrm{k} \pi) \cdot 10$ and it is the Diffraction Energy mechanism for all Space Levels of quantization which are the Energy Particles of monads only i.e.

Energy particles are then where issues $\rightarrow \mathrm{z}^{1 / \mathrm{w}}=|\mathrm{zo}|^{-} \mathrm{w} . \mathrm{Lv}=\rightarrow$ Energy Monads.

For $\sin .(\varphi+\mathrm{k} \pi) / \mathrm{w}=0$ then exists only the Real part of monad $, \mathrm{s}, \neq 0$, where $\varphi=-\pi \pm \mathrm{k} . \pi$ and then spaces, Massive particles are then where issues $\rightarrow \mathrm{z}^{1 /} \mathrm{w}=|\mathrm{zo}|^{-} \mathrm{w} . \mathrm{e}$ i. $(\varphi+\mathrm{k} \pi) / \mathrm{w}= \pm \mathrm{i} .(-\pi \pm \mathrm{k} \pi) \cdot 10$ and it is the Diffraction Massive mechanism for all Space Levels of quantization which are particles with mass ,i.e. the reaction to this motion only

Extending $\mathrm{Lv}=\mathrm{ei} \cdot(\mathrm{T} 2+2 \mathrm{k} \pi) \cdot \mathrm{b}=\mathrm{ei} \cdot(\mathrm{T2}+2 \mathrm{k} \pi) \cdot 10$ then

For base $\mathrm{e}=2,71828$ and $\mathrm{k}=0 \rightarrow \mathrm{Lv}=\mathrm{e}^{\wedge} \mathrm{i} \cdot( \pm \pi / 2) \cdot \mathrm{b}$ then $\mathrm{e}^{\wedge}(-15,7079)=1,78118 \cdot 10^{-} 7 \mathrm{~m}$

For base $\mathrm{e}=2,71828$ and $\mathrm{k}=1 \rightarrow \mathrm{Lv}=\mathrm{e}^{\wedge} \mathrm{i} \cdot(-3 . \pi / 2) \mathrm{b}$ then $\mathrm{e}^{\wedge}(-47,12389)=5,344.10^{-} 21 \mathrm{~m}$ 
For base $\mathrm{e}=2,71828$ and $\mathrm{k}=2 \rightarrow \mathrm{Lv}=\mathrm{e}^{\wedge} \mathrm{i} \cdot(-5 . \pi / 2) \mathrm{b}$ then $\mathrm{e}^{\wedge}(-78,5398)=\mathbf{8 , 9 0 6 . 1 0}{ }^{`} 35 \mathrm{~m}$ Planck`s Length . For base $\mathrm{e}=2,71828$ and $\mathrm{k}=3 \rightarrow \mathrm{Lv}=\mathrm{e}^{\wedge} \mathrm{i} \cdot(-7 . \pi / 2) \mathrm{b}$ then $\mathrm{e}^{\wedge}(-109,9956)=2,295 \cdot 10^{-} 48 \mathrm{~m}$ Layer Length . For base $\mathrm{e}=2,71828$ and $\mathrm{k}=4 \rightarrow \mathrm{Lv}=\mathrm{e}^{\wedge} \mathrm{i} .(-9 . \pi / 2) \mathrm{b}$ then $\mathrm{e}^{\wedge}(-141,372)=3,969.10^{-} 62 \mathrm{~m}$ Gravity Length . For base $\mathrm{e}=2,71828$ and $\mathrm{k}=5 \rightarrow \mathrm{Lv}=\mathrm{e}^{\wedge} \mathrm{i} \cdot(-11 . \pi / 2) \mathrm{b}$ then $\mathrm{e}^{\wedge}(-172,7876)=9,593.10^{-} 76 \mathrm{~m}$ Layer Length . For base $\mathrm{e}=2,71828$ and $\mathrm{k}=6 \rightarrow \mathrm{Lv}=\mathrm{e}^{\wedge} \mathrm{i} \cdot(-13 . \pi / 2) \mathrm{b}$ then $\mathrm{e}^{\wedge}(-204,2040)=7,155.10^{-} 89 \mathrm{~m}$ 1st Layer length . For base $e=2,71828$ and base $b=10$, then $e^{-78,5398}=8,906.10-35 \mathrm{~m}$ and divided by $\sqrt{3} \pi=1,616199.10-35 \mathrm{~m}$ Since cave is a versor then $\rightarrow$ Planck's Length

$\mathrm{Lp}=\mathrm{ei} \cdot(\pi 2+2 \mathrm{k} \pi) \cdot \mathrm{b}=\mathrm{e}-\mathrm{i} \cdot(5 \pi 2) \cdot \mathrm{b}=\mathrm{ei} \cdot(-5 \pi 2) \cdot 10=\mathrm{e}^{-} \cdot(78,5398) .=\mathbf{8 , 9 0 6} \cdot 10-35 \mathrm{~m}=\{\sqrt{3} \cdot \pi \cdot \mathbf{1 , 6 1 6 1 9 9} \cdot 10-35 \mathrm{~m}$ \}. i.e. Planck`s length, which is a universal constant, and is not becoming from any measurements, but of the Geometric logic of Caves, cavity, for the minimum element of Energy, the Photon .

Conservative Planck's constant L-Planck $=1,616.10-35 \mathrm{~m}=\sqrt{\mathrm{h}} . \mathrm{G}^{3}$ is consisted of the three compromising constants h,G,c, instead of geometry cave mould as above.

Lp = e i. ( $-5 \pi / 2$ ).10, which is based on the two natural constants e , $\pi$. [29-30] .

\subsection{The How Work is Quantized in Spaces and How it is into Equilibrium [40-41]}

In Mechanics, a force , $\mathbf{P}$, is said to do work , $\mathbf{W}$, if when acting on a body (something which has mass and has a resistance to any change of motion) there is a displacement, $\mathbf{d s}$, of the point of application in the Direction of the force, i.e. $\mathrm{W}=\mathrm{P}$. ds . For any direction with an angle, $\varphi$, between $\mathrm{P}$, ds then $\mathrm{W}=\mathrm{P} \cdot \mathrm{ds} \cdot \cos \varphi$ and for $\varphi=0,90 \mathrm{o}$ then it is $\mathrm{W}=\mathrm{P} \cdot \mathrm{ds}$, and $\mathrm{W}=0$.

In the three dimensional Euclidean Space P,ds are considered vectors, and their Dot-product $\mathrm{W}=\mathrm{P} . \mathrm{ds}$, define the geometric significance which is the directed Area, P.ds, while Cross-product W = Pxds defines the geometric significance, which is the directed perpendicular vector, to both vectors . Above definition defines work as quaternion, i.e. Dot-product W=P.ds to be the real part and Cross-product $\mathrm{W}=$ Pxds to be the imaginary part as work, $\mathrm{W}=[\mathrm{P} . \mathrm{ds}]+\mathrm{i} .[\mathrm{Pxds}]$.

Work is conserved as, as the Universal Principle of Virtual work on Geometry Primary dipole ,ds, W= $\oint$ F.ds , and as Energy is then Quantized, or converted, in Space monads , caves, cells, $\bar{x}=\overline{d s}=\lambda \cdot \mathrm{m}$, as pressure $\sigma, T$, the pressure is converted in caves as a Standing Electromagnetic Wave E,P which consists the Standing monad (Displacement current) and the moving Energy monad ( by altering the inner wavelength or Period of monad ) in Gravity`s field medium [MFMF] and is dissipated as Quaternion monads ( Particles or Waves, matter or vectors) as Forces ( displacements, masses, pressure etc . ) using modulus, coefficients, reactions to the motion and all other geometrical indices. Part of W, is kept as Dark-matter-energy Heap which is Rolling with lightvelocity on Gravity-field, thus causing the universe expansion.

Stability on monads is obtained by two ways .

1.Stability In-Out wavelength is obtained by the Isochronous Anti-Standing Electromagnetic Wave E,P which happens on Anti-cycloid, Evolute, and whirling, Curl, by the equality of the two transverse Complex Envelope displacements, Amplitudes, that consist the Standing monad (Coriolis force curl helix Displacement current in monads), or

2. Orbital stability is obtained by the Circular glue Motion of the Negative on Positive , where is created an Energy Wheel-Rim or a Cave, in where Negative breakage the Electron, of radius $\mathrm{r}$ and mass $\mathrm{m}$ is rolling on Cave`s Wall, and because executes a pressure then is positive .

\section{Remarks :}

Work as Energy is Quantized, converted, in Space monads, caves , cells, $\bar{x}=\mathrm{ds}=\lambda \mathrm{m}$, as pressure , $\sigma, \mathrm{T}$ , the pressure is converted in caves as a Standing Electromagnetic Wave E, P which consists the Standing monad ( Displacement current) and the moving Energy monad ( by altering the inner wavelength or Period of monad ) in Gravity`s field medium [MFMF] and is dissipated as Quaternion monads ( Particles or Waves , matter or vectors) as Forces ( displacements, masses, pressure etc.) using modulus, coefficients, reactions to the motion and all other geometrical indices.

2.The two $\bigoplus \ominus$ Particles, when in contact, equilibrium because of the two opposite and equal action forces F, - F, on their common point Because the two Forces, the Glued-Bond, are opposite and continually acting on this common point then according to Newton`s third law, This Common Point executes a circular motion with velocity $\mathrm{v}=\boldsymbol{v}=\sqrt{\boldsymbol{F} \cdot \frac{\boldsymbol{r}}{\boldsymbol{m}}}$ where, $\mathrm{r}=$ the radius of the $\bigoplus$ breakage and

$\mathbf{m}=$ the mass of the $\ominus$ breakage $=$ The reaction to the change of Velocity direction .

Generally, Monad NN $=\mathrm{N}(1) \leftrightarrow \mathrm{N}(2)$ is the dipole,$(\mathrm{P} 1 \leftrightarrow \mathrm{P} 2)$, or $[\{\mathrm{N}(\mathrm{P} 1) \leftarrow 0 \rightarrow(\mathrm{P} 2) \mathrm{N}\}]$ and it is the symbolism of the two opposite forces $(\mathrm{P} 1),(\mathrm{P} 2)$ which vibrate perpendicularly in monad (resonance cave) and are created as force at the edge points N1, N2 . Balancing of monads, Quaternions, happens on Evolutes cycloid, Anti-cycloid . F-18. 
For $\bar{v}=\bar{c}, \rho=X X^{\prime}=2 c \sqrt{ } r / g$, and $\operatorname{Spin} \bar{S}=\bar{V} X(T) \times X X^{\prime}=[g \cdot \sin \varphi] \cdot \rho=[g \cdot \sin \varphi] \cdot[2 c \sqrt{ } / g]=2 c \cdot \sin \varphi \cdot \sqrt{ } \mathbf{r g}=\mathbf{2 c} \sqrt{ } \mathbf{r g}$ . $\sin \varphi$, i.e Energy $c$, as Spin, is Unified with Space ,r, and as was shown by -GSSM- Mechanism.

The Cycloid , Anti-cycloid Evolute :

Cycloidal motion of equilibrium in moving monads by the Anti-cycloidal ( Evolute ).

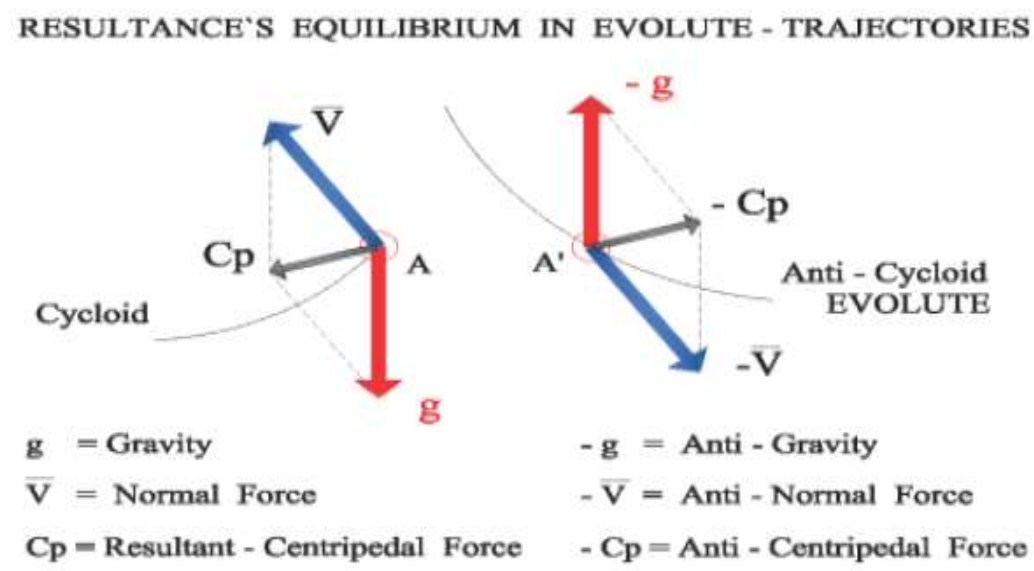

Figure 20.

Equilibrium of the Resultant Forces, in the Cycloid and as motion Wavelength, and of any Removal Monad . The Intrinsic Space (Wavelength $=[\lambda]$ ) is the Energy Quanta .The equilibrium of the Resultant forces is obtained by the above Cycloid, Anti-cycloid, Evolute, Forces or , by the Rotor and Spin in one Rim ,as was shown. In Rims is possible of forming velocities greater than that of light, and this because of different ,r, and the Tangential velocities are kept with the same angular velocity, the Spinning, unless spinning changes in such way to keep light-velocity constant .

Since, Energy of the first Wheel-Rim or Orbital is distributed to the couple of Electrons (2e) of the orbital then this is a Cycloid, Anti-cycloid, stability system of the closed systems. F-11

This is seemed in Extrema cases where Energy as Force is converted as Stress-Strain system or as Velocity-transverse velocity systems.

In the following figure, Work as Force x Displacement, is transformed into Principal-Stresses , which is transformed into Electromagnetic-Field, and for Zero surface is transformed into moving force which is Linear-momentum . For none-Zero surface Force is transformed into Work changed in time .

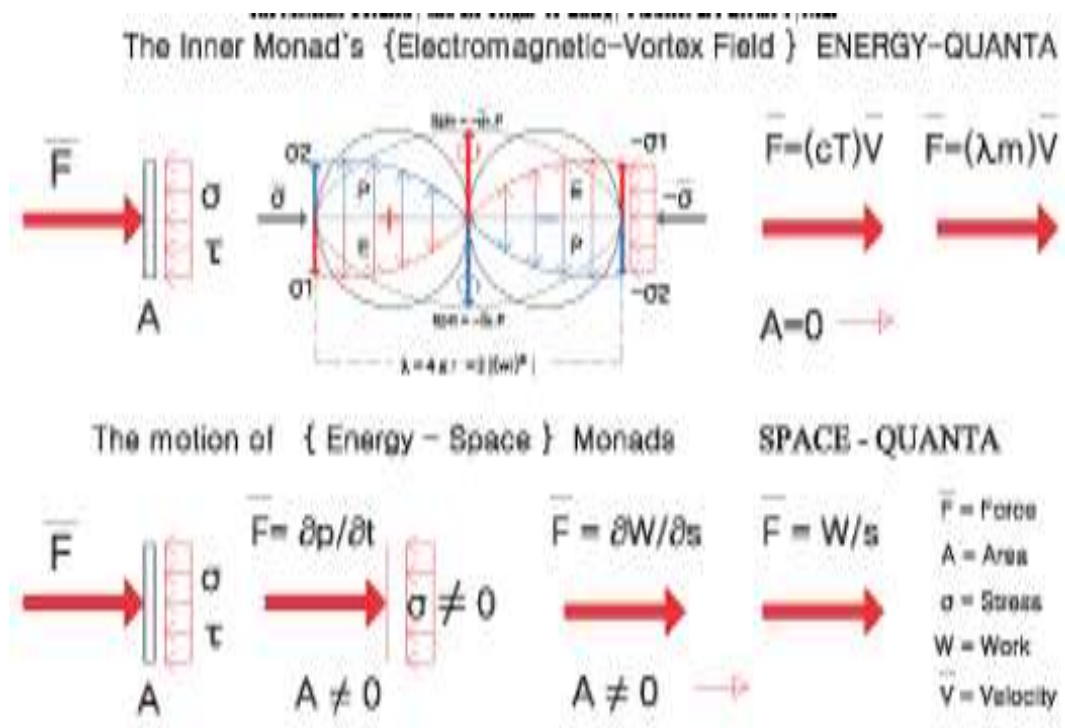

Figure. 21.

The Quantization of Work as discrete Force ( Energy ) = Electromagnetic field [E,P] . When Force F is applied on zero Surface, then is converted to velocity Vector which 
follows Quaternion rules, differently Mechanical .

\subsection{Remarks :}

The origin of Energy becomes, through the Principle of Virtual Displacements ,as the Work of the Inner Impulse distance of Space and Anti-Space embedded in all points of universe. The minimum Quantized Space, Quanta , $\mathrm{s}^{2}$, are in all quantized Spaces, i.e. Particles , [MFMF] Field, moving vectors, free velocity monads , Material Points and lines $\rightarrow$ Surfaces and bodies.

A comparison to Quantum-Mechanics is as follows :

Since each atom is consisted of the prior one , Plus energy of Electrons per Shell, then when energy is filling the Space Shell, a new Wheel-Rim of Proton is added as follows,

$\mathbf{n}=$ Orbital $\mathbf{l}=$ Shape-Orbital-Number $\mathbf{m}=$ Magnetic Quantum Number Pc=Number of Orbitals in the Subshell Orbital-Rims Positions Rc=Number of Rims , TE-Total Rims-Electrons , ES-Electrons per Subshell n-Orbital 1 -Number $\mathbf{m}$-Number Rc- Number E-per Shell S-Notation ES TE

00 Nucleus-Rotor $01+\uparrow 11$

1.. s 00 P1 $21[+\uparrow-\downarrow] 62$

2.. p $1-\mathbf{1 , 0}, \mathbf{0 1}, 2 \mathrm{P} 181[+\uparrow-\downarrow] 68$

$2 \mathrm{P} 1+\mathrm{P} 22[+\uparrow-\downarrow] 1010$

3.. d $2-2,-\mathbf{1}, 0$, +1, +2 2P1+2P2 $182[+\uparrow-\downarrow] 1018$

$2 \mathrm{P} 1+2 \mathrm{P} 2+\mathrm{P} 34[+\uparrow-\downarrow] 1436$

4.. f $3-3,-2,-\mathbf{1}, \mathbf{0},+\mathbf{1},+2,+32 \mathrm{P} 1+2 \mathrm{P} 2+2 \mathrm{P} 3324[+\uparrow-\downarrow] 1454$

$2 \mathrm{P} 1+2 \mathrm{P} 2+2 \mathrm{P} 3+\mathrm{P} 46[+\uparrow-\downarrow] 1886$

5.. g $4-4,-3,-2,-\mathbf{1}, \mathbf{0},+\mathbf{1},+2,+3,+42 \mathrm{P} 1+2 \mathrm{P} 2+2 \mathrm{P} 3+2 \mathrm{P} 4506[+\uparrow-\downarrow] 18118$

$2 \mathrm{P} 1+2 \mathrm{P} 2+2 \mathrm{P} 3+2 \mathrm{P} 4+\mathrm{P} 58[+\uparrow-\downarrow] 22168$

6.. h $5-5,-4,-3,-2,-\mathbf{1}, \mathbf{0},+\mathbf{1},+2,+3,+4,+5$ 2P1+2P $2+2 \mathrm{P} 3+2 \mathrm{P} 4+2 \mathrm{P} 5728[+\uparrow-\downarrow] 22218$

where,

$\mathrm{n}=$ Principle Quantum Number $\mathrm{n}=1,2,3, . . \mathrm{n}$

$1=\mathrm{n}-1=$ Orbital Quantum Number $1=0,1,2,3, . . \mathrm{n}-1$, which is the number of orbital shapes .

$\mathrm{ml}=2 \mathrm{l}+1=2 \mathrm{n}-1$ values $(\mathrm{it}$ is magnetic Quantum Number values .

ms $=[+\uparrow-\downarrow],($ The Spin Quantum Numbers $+1 / 2-1 / 2=0$, which is a Stable - State .

The New Position-Proposal of Material-Geometry :

The Idea of Positions and the Glued-Bond-Motion of opposites, applied on the Geometrical-Atom-RimConstruction for a continual action is as follows .

1. Continuous action of Negative $\Theta$, on One Positive $\Theta$, is equivalent to a Circular motion of $\Theta$ to $\bigoplus$.

2. Continuous action of Negative $\Theta$, on Two Positive $\bigoplus \bigoplus$ of glued bond, is equivalent to a Circular-and Other of one-step longer motion of $\ominus$ to $\bigoplus \bigoplus$, or Circular motion on the Common-Conduct.

3. Continuous action of Negative $\ominus$, on Three Positive $\bigoplus \bigoplus \bigoplus$ of glued bond, is equivalent to a Circular-and Other of two-step longer motion of $\ominus$ to $\bigoplus \bigoplus \bigoplus$, or Circular and one-step longer motion on the Common-Conduct .

4. Continuous action of Negative $\ominus$, on Four Positive $\bigoplus \bigoplus \bigoplus \bigoplus$ of glued bond , is equivalent to a Circular and Other of three-step longer motion of $\ominus$ to $\bigoplus \bigoplus \bigoplus \bigoplus$, or Circular and two-step longer motion on the Common-Conduct

5. Continuous action of Negative $\ominus$, on Five Positive $\bigoplus \bigoplus \bigoplus \bigoplus \bigoplus$ of glued bond , is equivalent to a Circular and Other of four-step longer motion of $\ominus$ to $\bigoplus \bigoplus \bigoplus \bigoplus \bigoplus$, or Circular and three-step longer motion on the Common-Conduct, and so on as below Table .

i.e. in W-Rim.5 of Nucleus exist $\rightarrow 54$ Protons in Nucleus $\rightarrow 6$ Orbitals of 6 Protons $\rightarrow 3$ Sub-Rims available $\rightarrow 2$ Containers of 18 Protons each and 36 Protons in both $\rightarrow 54$ the Total number of Protons $\rightarrow 18^{\circ}$ is the Sub-Rim angle, $\rightarrow 10^{\circ}$ is the demanded Stable-System-Helix-angle.

The Total Quantity of Quantized-Energy stored in Rim.5 is as before found as E $=[\mathrm{E} 1+\mathrm{E} 14+\mathrm{E} 19+\mathrm{E} 116+\mathrm{E} 125]$ All in Table below are geometrically shown in Figure 18.

Rim Protons Orbitals Available Available Protons Sub-Rim Available Protons Total-Protons Angle No Number Total-Rims Sub-Rims in-Rims Number Protons in Nucleus in Rims $\varphi=180 / R$

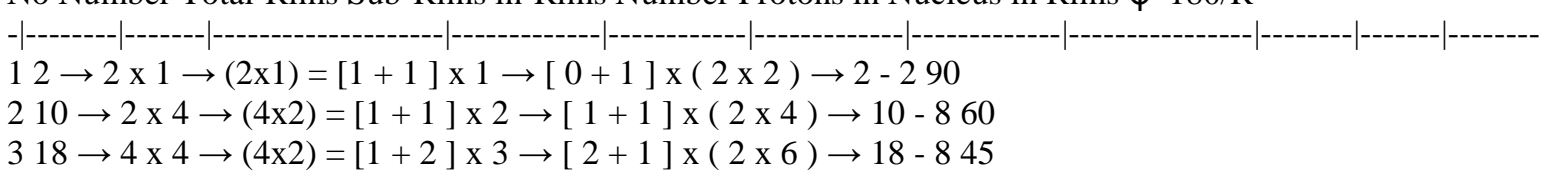


$436 \rightarrow 3 \times 6 \rightarrow(3 \times 6)=[1+2] \times 3 \rightarrow[3+1] \times(2 \times 8) \rightarrow 36-1822,5$

$\mathbf{5 5 4} \rightarrow 6 \times 6 \rightarrow(3 \times 6)=[\mathbf{1}+\mathbf{2}] \times \mathbf{3} \rightarrow[\mathbf{4}+\mathbf{1}] \times(2 \times 10) \rightarrow \mathbf{5 4}-18 \mathbf{1 8}$

$686 \rightarrow 4 \times 8 \rightarrow(4 \times 8)=[1+2] \times 4 \rightarrow[5+1] \times(2 \times 12) \rightarrow 86-3215$

$7118 \rightarrow 8 \times 8 \rightarrow(4 \times 8)=[1+2] \times 4 \rightarrow[6+1] \times(2 \times 14) \rightarrow 118-3212,5$

$8168 \rightarrow 5 \times 10 \rightarrow(5 \times 10)=[1+2] \times 5 \rightarrow[7+1] \times(2 \times 16) \rightarrow 168-5011,2$

$9218 \rightarrow 10 \times 10 \rightarrow(5 \times 10)=[1+2] \times 5 \rightarrow[8+1] \times(2 \times 18) \rightarrow 218-5010$

$10268 \rightarrow 6 \times 12 \rightarrow(6 \times 12)=[1+2] \times 6 \rightarrow[9+1] \times(2 \times 20) \rightarrow 290-729$

6.. Wheel Rims Order :

It was shown that The Possible Repetitive Permutations for Moulds and Units are 2. $\square \square \square \square \square \square \square \square \square \square=2 . \mathrm{M}^{2}$, for every Mould $=$ Space $=$ Any Wheel Rim $($ W-R $)$. Applying above logic for Nucleus-Protons then ,

$\mathrm{W}-\mathrm{R} 1$ contains $\rightarrow 2=2$ Positions, or $\mathrm{W}-\mathrm{R} 1=2\left[1.1^{2}\right]$

$\mathrm{W}-\mathrm{R} 2$ contains $\rightarrow 2+1.8=10$ Positions, or W-R2 $=2\left[1.1^{2}+1.2^{2}\right]$

$\mathrm{W}-\mathrm{R} 3$ contains $\rightarrow 2+2.8=18$ Positions, or $\mathrm{W}-\mathrm{R} 3=2\left[1.1^{2}+2.2^{2}\right]$

$\mathrm{W}-\mathrm{R} 4$ contains $\rightarrow 2+2.8+1.18=36$ Positions, or $\mathrm{W}-\mathrm{R} 4=2\left[1.1^{2}+2.2^{2}+1.3^{2}\right]$

$\mathrm{W}-\mathrm{R} 5$ contains $\rightarrow 2+2.8+2.18=54$ Positions, or $\mathrm{W}-\mathrm{R} 5=2\left[1.1^{2}+2.2^{2}+2.3^{2}\right]$

$\mathrm{W}-\mathrm{R} 6$ contains $\rightarrow 2+2.8+2.18+1.32=86$ Positions, or $\mathrm{W}-\mathrm{R} 6=2\left[1.1^{2}+2.2^{2}+2.3^{2}+1.4^{2}\right]$

$\mathrm{W}-\mathrm{R} 7$ contains $\rightarrow 2+2.8+2.18+2.32=118$ Positions, or $\mathrm{W}-\mathrm{R} 7=2\left[1.1^{2}+2.2^{2}+2.3^{2}+2.4^{2}\right]$

$\mathrm{W}-\mathrm{R} 8$ contains $\rightarrow 2+2.8+2.18+2.32+1.50=168$ Positions , or W-R $8=2\left[1.1^{2}+2.2^{2}+2.3^{2}+2.4^{2}+1.5^{2}\right]$

$\mathrm{W}-\mathrm{R} 9$ contains $\rightarrow 2+2.8+2.18+2.32+2.50=218$ Positions, or $\mathrm{W}-\mathrm{R} 9=2\left[1.1^{2}+2.2^{2}+2.3^{2}+2.4^{2}+2.5^{2}\right]$

$\mathrm{W}-\mathrm{Rc}$ contains $\rightarrow 2+2.8+2.18+2.32+2.50+\ldots .2 . \mathrm{R}^{2} \mathrm{c}=$ ccccccc Positions, or

$\mathrm{W}-\mathrm{Rc}=2[1+8+18+32+50+\ldots]=.2+4\left[2^{2}+3^{2}+4^{2}+5^{2}+\ldots . \mathrm{R}^{2} \mathrm{c}\right]=2 . \mathrm{M}^{2} 1+4 \cdot\left[\mathrm{M}^{2} 2+\mathrm{M}^{2} 3+\mathrm{M}^{2} 4+\ldots \mathrm{M}^{2} 1+\mathrm{c}\right]$

\subsection{The Nucleus Structure :}

The equilibrium of the Resultant forces in Monads is obtained by the Cycloid, Anti-cycloid, Evolute , Forces or, by the Rotor and Spin in Rims, as was shown. The two Particles, when in contact $\bigoplus \Theta$, equilibrium $\bigoplus \leftrightarrow \Theta$, because of the two opposite and equal action forces F, - F , on their common point . Because the two Forces, the Glued-Bond, are opposite and continually acting on this common point then according to Newton`s third law this Common Point executes a Circular-motion with velocity $\bar{v}$. Adding positive $\bigoplus$ particles then the negative particle $\ominus$ executes a , coupler curve, dependent on the curve of rotation of the Negative particle . Because the Evolute is the Coupler-Curve of a curve, therefore , Euler-Savary equation gives the radius of curvature and the center of curvature of this coupler curve and the Cube of Stationary Curvature.

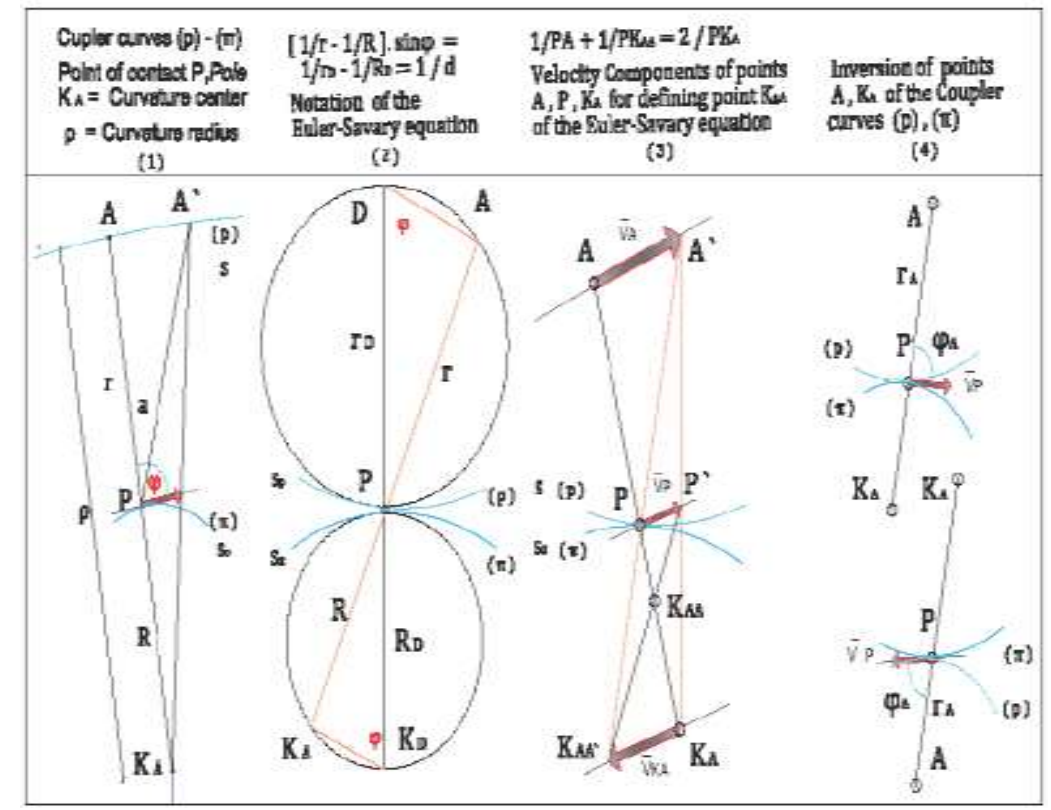

Figure. 22.

In (1) A point A on Coupler-curves (p), ( $\pi$ ) define the point of curvature KA, the Instaneous point $\mathbf{P}$, the pole on (Ti) .

In (2) is the case of point A lying on radius of curvature of polar path (point D) where then the paths of points $A$ in $\mathrm{S}$ system have the Instaneous center of curvature KA on the fixed system So.

In (3) The Velocity Instaneous center, for curvature point KA in So system is point KAA . 
In (4) The two points A, KA of Coupler-curves (p), (Ti), follow the inversed motion .

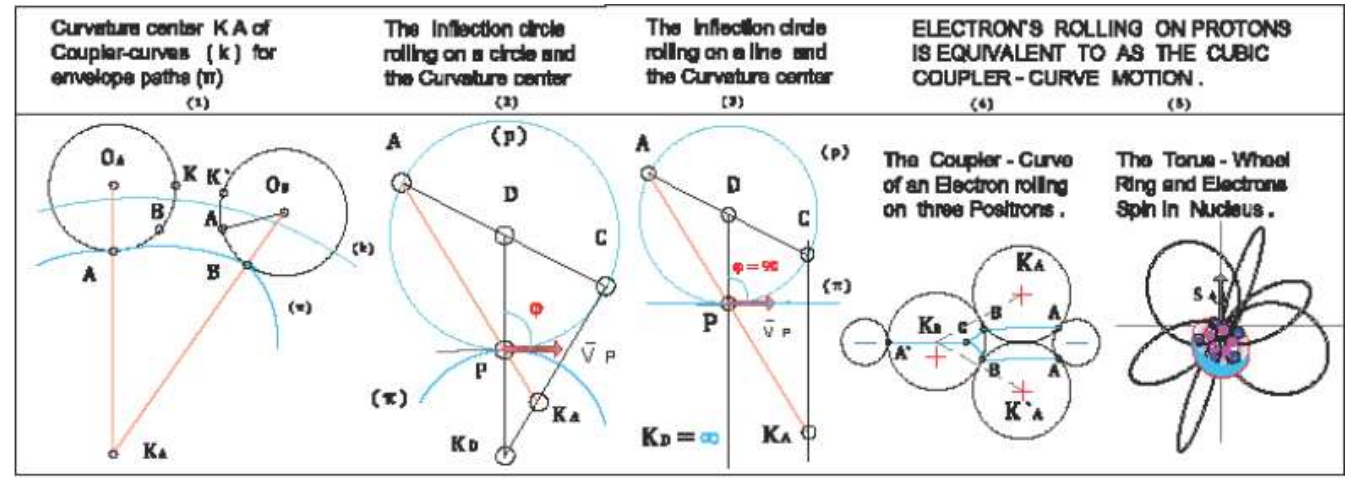

Figure. 23

In (1) Point of curvature KA of (k) path on the constant envelope ( $\pi$ ) coincides with that of (k) .

In (2) The rolling of (p) circle on (Ti) circle defines the curvature center KA on AP produced

In (3) The rolling of (p) circle on ( $\pi$ ) line defines the curvature center KA on AP produced on parallel to DP, from point $\mathrm{C}$

In (4) Electron $\ominus$ rolling on three Protons $\bigoplus$ is drawing the two branches of ABCA '

One is the straight line, first degree, the other is a circle, second degree. The COSC curve

At point B , Concavity point, Evolute changes sign while at $\mathrm{C}$ point, Inflection point , direction.

In (5) Wheel-Rims of Nucleus appear as magnetic dipoles in magnetism .

A.. The Coupler Curves .

Geometry :

Let $\mathrm{A}$ be a point on a Plane System ,S, rolling on the fixed system ,So, as in Fig-22.1 KA is the center of curvature, the Instaneous center on the fix system . P is the Instaneous center of curvature on the fix curve So (the pole $\mathrm{P}$ ), (p), (T) are the coupler curves on $\mathrm{S}$, So $\mathrm{u}=$ The translational velocity of pole $\mathrm{P}$ equal to $\mathrm{ds} / \mathrm{dt}=$ $\mathrm{AA} \% \mathrm{dt} \mathrm{w}=$ Angular velocity of pole $\mathrm{P}$ equal to $\mathrm{dr} / \mathrm{dt}=\mathrm{d}\left(\mathrm{APA}^{\top}\right) / \mathrm{dt}$ and for $\mathrm{d}=\mathrm{u} / \mathrm{w}$ then , Euler-Savary equation is $[1 / \mathrm{rD}-1 / \mathrm{RD}] \sin \varphi=1 / \mathrm{d}$

When point $P$ lies on the radius of curvature of Polar path $(\varphi=90)$ then $\sin \varphi=1$ and from Fig-22.2

$[1 / \mathrm{rD}-1 / \mathrm{RD}]=1 / \mathrm{d}$ and issues $\mathrm{r}=\mathrm{rD} \cdot \sin \varphi$ and $\mathrm{R}=\mathrm{RD} \cdot \sin \varphi$

i.e.

curvature

The trajectories of points $A$ on the circumference of circle radius $\square \square$, have their center of on circumference of circle of radius $\square \square$.

Motion :

The motion of curves $(\mathbf{p}),(\pi)$ is in Fig-22.3 Let vA, $\mathrm{p}$, to their systems .

For system $\mathrm{S}$ the curvature center KA, the Instaneous center, is found from the intersection of $\mathrm{A}^{`} \mathrm{P}^{`}$ and $\mathrm{AP}$. For system So the curvature center KAA, the Instaneous center of KA on fixed system ( $\pi$ ) , is found from the intersection of $\mathrm{P}^{`}$ KAA` and PKA . From the above similar triangle KAAA`, KAPP` exists ,

$(\mathrm{KAA} / \mathrm{PA})=\left(\mathrm{KAA}^{\prime} / \mathrm{P}^{\prime} \mathrm{A}^{\prime}\right)=\left(\mathrm{KAA}^{\prime} \mathrm{A}^{\prime} / \mathrm{PKAA}^{\prime}\right)=\mathrm{KA} \mathrm{KAA} / \mathrm{P} \mathrm{KAA}$ or $\{\mathrm{KAA} / \mathrm{PA}\}=\{\mathrm{KA} \mathrm{KAA} /$ KAAP $\} \ldots$ (b)

i.e. The Points A, KAA are harmonically divided by the points $\mathrm{P}, \mathrm{KA}$ and exists $1 / \mathrm{PA}+1 / \mathrm{P} \mathrm{KAA}=2 / \mathrm{PKA}$

Inversing the two Systems by considering fixed system ,So, rolling on ,S, as in Fig-22.4 then, [ 1/rA $1 / \mathrm{RA}] \sin \varphi \mathrm{A}=1 / \mathrm{d}$ and [ $\left.1 / \mathrm{rA}^{`}-1 / \mathrm{RA}^{\prime}\right] \sin \varphi^{\prime} \mathrm{A}=1 / \mathrm{d}$ where in both cases issues ,

$(\mathrm{PKA}-\mathrm{PA}) /(\mathrm{PKA} . \mathrm{PA})=-\left(\mathrm{PK}^{\prime} \mathrm{A}-\mathrm{PA}^{\prime}\right) /\left(\mathrm{PKA}^{\prime} . \mathrm{PA}^{\prime}\right)$ or $(1 / \mathrm{PA}-1 / \mathrm{PKA})=\left(1 / \mathrm{PKA}^{\prime}-\mathrm{PA}^{\prime}\right)=1 / \mathrm{d} \ldots(\mathrm{b})$

The Path of the Instaneous-center of curvature, $\square \square$, on $(\mathrm{k})$, ( $(\pi)$ coupler envelope curves is proved that, During the rolling of curve $(\mathrm{k})$ of system $\mathrm{S}$ and the fixed to it envelope $(\pi)$, then the Instaneous-center of curvature and those of the constant envelope ( $\pi$ ) , coincides to the Instaneous-center of curvature KA of (k) as in Fig-23.1 The center D, of a Rolling circle (p) on another circle ( $\pi$ ), executes a circular motion with KD as center which coincides with the center of curvature of the second circle. Because angle $\varphi=90^{\circ}$, then for every point A on (p) exists a center of curvature KA on AP and C Kp as in Fig-23.2

During the rolling of a circle (p) on ( $\pi$ ) line , then the corresponding Instaneous-center of curvature KA of any point $\mathrm{A}$ is the common point of intersection of AP produced and the parallel to DP from point $\mathrm{C}$ and the Instaneous-center of curvature $\mathrm{KD}$ for point $\mathrm{D}$ is in infinite and $\mathrm{KD}=\infty$. The Euler-Savary equation 
involves the four points A, P, KA, KAA lying on the path normal. Equation (b) may be written in the form PA / $\mathrm{AKAA}=\mathrm{A} \mathrm{KAA} / \mathrm{AKA}$ and is recognized that AKAA is the mean proportional between PA and KAA .

The Cubic of Stationary curvature :

Euler-Savary formula apply to the analysis of a mechanism in a given position and vicinity . It gives also the radius of curvature and the center of curvature of a couple-curve. Because couple-curve ( Path $\leftrightarrow$ Evolute) isthe equilibrium of any moving system, then Complex-plane is involved and the E-S geometrical equations

$(1 / \mathrm{PA}-1 / \mathrm{PKA})$ i.ei $\varphi=\mathrm{h}[1 / \mathrm{PA}-1 / \mathrm{PKA}]=\mathrm{h} .(\mathrm{d} \varphi \mathrm{ds})$ and for the homothetic motion $(\mathrm{h}=1)$ then,

$$
\text { 1PA -1PKA = 1P KAA }(\mathrm{d} \varphi \mathrm{ds})
$$

Equation (c) is that of Rhodonea Hypocycloid curves .

The Inflation circle shows the location of coupler points whose curves have an infinite radius of curvature, i.e. on inflection circle lie all centers of curvature of System curves and which these are rolling on inflection point on the envelope . (Envelope here are the two or more surfaces in direct contact ). The Cubic of Stationary curvature [COSC] indicates the location of coupler points that will trace segments of approximate circular arcs . In Geometry, the rolling of a circle, on a circle and or on a line is likewise to Mechanism as , a Negative particle, Electron, on a Positive particle, Proton, or on many Protons, so the Wheel-Rims represent the , COSC in Mechanics .

Figure Fig-23.4 demonstrates the two symmetrical paths on, arc circles and on lines, of the Electron`s Direct-Contact-Point motion, where Electron represents the moving System, and Proton -Protons represent the fixed System .The Geometrical construction of COSC .

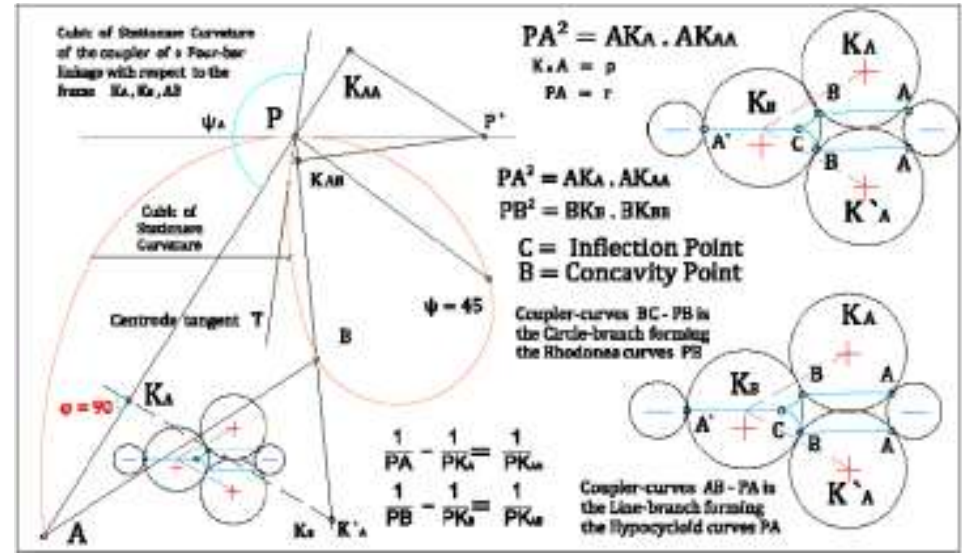

Figure. 24.

The Cubic of stationary curvature, COSC, of the coupler of a four bar linkage with respect to the fixed System $\mathrm{S} \rightarrow \mathrm{KA}, \mathrm{K} ` \mathrm{~A}, \mathrm{~KB}, \mathrm{~A}$.

In First Coupler-curve $\mathbf{A B}$, Electron $\Theta$ is rolling on three Protons $\bigoplus$ and is drawing branch $A B-\mathrm{CA}^{`}$, Spherical circle, is forming the Rhodonea -Hypocycloid curves.

In Second Coupler-curve AB , Electron $\ominus$ is rolling on three Protons $\bigoplus$ and is drawing branch BCA ', two Spherical circles, is forming the Archimedean Hypocycloidal-Spiral

At point B , COSB changes direction and is formed the symmetrical Pedal of the epicycloid while at Inflection Point $\mathrm{C}$, changes only the sign .

In Figure.24, point A is rolling on circle ( $\mathrm{KA}, \mathrm{KBA}$ ) from point $\mathrm{A}$ to $\mathrm{B}$ executing the circular arc AB . Points $\mathrm{KA}, \mathrm{KB}$ are the centers of curvature for points $\mathrm{A}, \mathrm{B}$. Point $\mathrm{A}^{\prime}$ is the end of arc BA`. Mechanism is a Four-bar linkage for points A, B , C , A` for the first position shown .

Step 1.. Locate the instaneous center P from the intersection of AKA, BK'A

Step 2.. Determine the direction of the common tangent PT to the centrodes by constructing the inflection circle of diameter PP and by $90^{\circ}$ rotation of its diameter. The inflection circle passes through the instaneous center P and the two points KAA, KAB on lines KAA and K'AB respectively. Euler-Savary equation gives ,AKAA = $(\mathrm{PA})^{2} \mathrm{AKA} \mathrm{PKAA}=\mathrm{PA}-\mathrm{AKAA}$ and $\mathrm{BKAB}=(\mathrm{PB})^{2} \mathrm{~K} ` \mathrm{AB} \mathrm{PKAB}=\mathrm{PB}-\mathrm{BKAB}$

Step 3. Confirmation of $\mathrm{PA}, \mathrm{PB}$ that is done using the values of angles $\psi$ and $\mathrm{X}, \mathrm{Y}$ functions, and also the cubic equation for points $\mathrm{A}, \mathrm{B}$. Equations are $1 \mathrm{X} \cdot \sin \psi \mathrm{A}+1 \mathrm{Y} \cdot \sin \psi \mathrm{A}=1 \mathrm{PA}$ and $1 \mathrm{X} \cdot \sin \psi \mathrm{B}+1 \mathrm{Y} \cdot \sin \psi \mathrm{B}=$ ${ }^{1} \mathrm{~PB}$ and for $\mathrm{PA}=\mathrm{PB}=\infty$ then $1 \mathrm{X} \cdot \sin \psi \mathrm{A}=-1 \mathrm{Y} \cdot \sin \psi \mathrm{A}$ and $1 \mathrm{X} \cdot \sin \psi \mathrm{B}=-1 \mathrm{Y} \cdot \sin \psi \mathrm{B}$

\section{Remarks :}


a.The diameter of Electron is nearly $2.10^{3}$ smaller than that of Proton, therefore angle $\psi$ is very small and in vicinity then corresponds to an angle $\varphi=0,318^{\circ}$.

b.At point $B$ there is a cusp so the intersection of the coupler curve with the cubic is a point of maximum or minimum curvature . Coupler point for B being very close to the fixed centrode of the two Protons will describe circular arcs as this happens to Helium element which has two Protons .

c.At pole $\mathrm{P}$ the two tangents are the centrode Normal and the centrode Tangent for the inflection circles. d.On arc AB, COSB draws PB Pedal, On arc BC draws the Symmetrical-negative Pedal because changes the direction and, and from Inflection Point C, On arc CA` the perpendicular CA` Pedal .

\section{STABILITY:}

According to Mechanics, Stability of a Wheel Rim is obtained through spinning in shortest or longest axis , which is that through the center of mass, elsewhere when with the Resultant of Spins to be zero.

In a Principle Axis-Frame, Space-configuration follows the shape of a Sphere as $\mathrm{Sx}^{2}+\mathrm{Sy}^{2}+\mathrm{Sz}^{2}=$ constant in the three dimensional Space, occupied by the angular momentum vector and rotating about the longest axis , either an Ellipsoid Sx ${ }^{2} I x \cdot x+S x^{2} I x \cdot x+S x^{2} I x \cdot x=$ constant , and rotating about the shortest axis as this happens to the Hydrogen-Rotor in W-Rim.1. , [25]

\section{i.e. Bonds of Hydrogen with that Atom which have Zero Resultant magnetic Spin $[\square \square=0$ ] are Stable.} a.. Wheel-Rims Rotating Unbalance :

Unbalance of rotating Electrons, is a common source of vibration excitation. Considering a Spring system constrained to move in the vertical direction $(\mathbf{Z}$ axis) and excited by a rotating electron is unbalanced . Electron mass , $\mathbf{m}$, in Rim`s mass ,M, of eccentricity ,e, and rotating with angular velocity ,w, in constant non-rotating mass M-m then gives equation of motion $[\mathrm{M}-\mathrm{m}] \ddot{\mathrm{x}}+\mathrm{m} \mathrm{d}^{2} \mathrm{dt}^{2}(\mathrm{x}+\mathrm{e} \cdot \operatorname{sinwt})=-\mathrm{k} \mathrm{x}-\mathrm{c} \dot{\mathrm{x}}$, which has the SteadyState Solution $X=\operatorname{mew}^{2} \sqrt{ }(\mathrm{k}-\mathrm{Mw} 2) 2+(\mathrm{cw})^{2}$ and $\tan \varphi=\mathrm{cwk}^{-\mathrm{Mw}^{2}}$, where $\mathrm{X}=$ the Resonant amplitude , and by $\mathrm{wn}=$ The Natural frequency,$\zeta=$ The Dumping factor of the system , then reduces to the Non - dimensional form,$\frac{\mathbf{M}}{\mathbf{m}} \frac{\mathbf{X}}{\mathbf{e}}=\frac{(\mathrm{w} / \mathrm{wn})^{2}}{\sqrt{\left(1-(\mathrm{w} / \mathrm{wn})^{2}\right)^{2}}+(2 \zeta \cdot \mathrm{w} / \mathrm{wn})^{2}}$, and $\tan \varphi=\frac{2 \zeta(\mathrm{w} / \mathrm{wn})}{1-(\mathrm{w} / \mathrm{wn})^{2}} \ldots . . . .$. (a) which is

A Rotor Balancing machine depended on simultaneously translation and angular motion .

\section{b.. Stability of Whirling of monads :}

Monads travel with a velocity, $\mathrm{v}$, which is analyzed to two perpendicular velocities $\overline{\mathrm{v}} 1, \overline{\mathrm{v}} 2$ (The thrusts) figure.5, following the cycloid axis , shaft (1)-(2) with center $\mathrm{O}$, and are synchronous and acquire equal speed of rotation, w, and equal to the whirling speed $\theta$ and thus exist rotational $\theta=\mathrm{w}$.

Equilibrium of whirling presupposes the balancing of the Space, Anti-space opposite and equal motion, as SB and DB .

On intergrading the speed is obtained $\varphi=w t$, where $\varphi$ is the phase angle between moving shaft center $\mathrm{O}$, of AA , OA is the eccentricity from (1)-(2) axis , and $\mathrm{O}$ the geometric center of , $|\bar{v}|=r \rightarrow R$ which is constant . Since $\theta=\mathrm{w}=$ constant then $\theta=\dot{\mathrm{r}}=\ddot{\mathrm{r}}=0$ and the problem reduces to that of one degree of freedom with mass $\mathrm{m}=\varepsilon$ $=\mu=1$ for monads. By using the general equations of whirling then $\rightarrow\left(\mathrm{km}-\mathrm{w}^{2}\right) \cdot \mathrm{R}=\mathrm{e} \cdot \mathrm{w}^{2} \cdot \cos \varphi, \mathrm{cm} \cdot \mathrm{w} \cdot \mathrm{R}=$ e. $\mathrm{w}^{2} \cdot \sin \varphi \leftarrow$ and by division equation is obtained, $\tan \varphi=(\mathrm{Cm} \mathrm{w} \cdot \mathrm{R}):\left(\mathrm{km}-\mathrm{w}^{2}\right)=[2 \zeta \mathrm{wwc}]:\left[1-(\mathrm{wwc})^{2}\right]$ where, $\mathrm{wc}=\sqrt{\mathrm{k}} / \mathrm{m}$ is the critical speed, $\zeta=\mathrm{CCc}, \mathrm{wc}=\mathrm{n}=\sqrt{\mathrm{k} / \mathrm{m}}=$ The Critical frequency or Natural Electron-frequency, and $\mathrm{e}=\mathrm{OA}^{\prime}$ is the eccentricity where from Pythagoras theorem the Phase, $\varphi$, is

$\cos \varphi=\left(\mathrm{km}-\mathrm{w}^{2}\right): \sqrt{ }(\mathrm{km}-\mathrm{w} 2) 2+(\mathrm{cm} . \mathrm{w})^{2}$ and the Amplitude equation becomes, for Resonant Amplitude

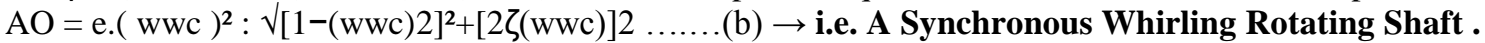

\section{Remarks :}

Equation indicates that eccentricity line $\mathrm{e}=\mathrm{OA}^{`}$ leads the displacement line $\mathrm{OA}=0 \rightarrow \mathrm{R}$ by the phase $\varphi$ , which depends on the amount of damping and the rotation speed ratio wwc, and in critical ratio $\square \square=\sqrt{\mathrm{k}} / \mathrm{m}$ frequency in lateral vibration, the amplitude is restrained by the damping $\varepsilon=\mu=1$. Point $\mathrm{O}$ is always on axis ,shaft, and point A rotates about it on circle of radius OA. The existing force is equal to $\rightarrow$ m.e. $w^{2}=m \cdot O A \cdot w^{2}$. In wavelength`s monad $\mathrm{AO}: \mathrm{e}=\mathrm{AO}: \mathrm{OA}^{`}=\mathrm{wwc}^{2}{ }^{2} \sqrt{ }[1-(\mathrm{wwc}) 2]^{2}+[2 \zeta(\mathrm{wwc})] 2$ and for $\zeta=0$ then $\mathrm{AO}: \mathrm{AO}^{`}=($ wwc) 2: [1-(wwc) ${ }^{2}$ ] with static Unbalanced and because of the Space, Anti-space equilibrium in monads $\mathrm{wc}=\sqrt{\mathrm{k}} / \mathrm{m}$, then $\mathrm{AO}: \mathrm{AO}^{`}=( \pm \mathrm{i})$, i.e. Space , Anti-space are interchanged having equal eccentricity because for $\mathrm{AO}: \mathrm{AO}^{`}=1 \rightarrow(\mathrm{wwc})=1 / \sqrt{2}$ which happens at point $(1)$ of Stationary Electromagnetic wave . Resonant Amplitude AO of eccentricity ,e, is perpendicular to Rim and for Rotor is $\mathbf{Z}$, axis .

\section{c.Static and Dynamic Unbalance of Rims :}

The unbalance is when all masses lie in a Single Plane as the case of the thin Wheel-Rim or Rotor disk , the resultant Unbalance is a single radiant force which is rolling the Rim to a Position where the heavy point is 
directly below the axis and since can be appeared, detected, without Spinning the Wheel-Rim is then called SU $=$ Static-Unbalance .

When the unbalance appears in more than one Plane the Resultant is a Force and a rocking Moment which is referred as $\mathbf{D U}=$ Dynamic-Unbalance and is appeared by the Rotor-Spinning .

\section{Stability Of Nucleus :}

1.. On one Proton, an Electron is sufficient to produce rotation, which rotation is originated from the continually equal and opposite Centrifugal , CF , and Centripetal , CP , forces . Infinite positions are possible for this equilibrium .

2.. On two Protons, an Electron is sufficient to produce rotation following a steady circular orbit, i.e. It is the first Wheel Rim , a solid ring, which equilibrium angular Momentum in four Modes of rotation ,the three in $\mathrm{X}, \mathrm{Y}, \mathrm{Z}$ axis and fourth on Spin direction which is perpendicular to its plane .The Rotor is a ring which rotates like a flipped coin about two different diameters perpendicular to each other, therefore the Dynamic-structure of a nucleus Wheel-Rim is like the Synchronous Whirling Rotating Shaft .

3.. On three or , N , Protons, three or , N , Electron are sufficient to produce rotation and three or , N, on Rotor . The dynamical nucleus is composed of the Rotor and the concentric Spherical or Hypocycloid Rhodonea Pedal-Shells.

A nucleus is held together by the linkages created by the formation of Spin pairs of the Wheel-Rims on Rotor Spin and this for conservation of System`s Total-energy.

The Wheel-Rims of the , Helium-Modulus-Rotor, rotate and create the Dynamic appearance, which are held together through the repulsion of the Proton forces. Neutrons Spin-pairs outside of the W-Rims are held by their attraction to the Rotors spin. The moving Electrons around the Nucleus protons, create an Electromagnetic field.

4.. In all Dynamic Systems energy is conserved, therefore Work produced by the Rotor and Wheel-Rims is Conserved as Torsional momentum. The dynamic stability of the nucleus torsional-momentum is assured by the Resultance of the partial momentums of the wheel-Rims which have their envelope centers of curvature on Euler-Savary coupler-curves. The determination of Nucleus curvature centers is done either by the Bobillier method, or with Euler-Savary Cubic-of-Stationary-curvature equation .

5.. Nucleus torsional momentum may be considered as the natural frequency of a uniform line-rod, T,in torsional oscillation with one end fixed in nucleus and the other end free. Applyıng the boundary conditions when $x=0, \theta=0, x=T$, torque $=0$ or $\partial \theta / \partial x=0$ then is proved that , cosw. $\sqrt{\rho} / G T=0$ which is satisfied by the angles $[w n \sqrt{ } \rho / G] . T=\pi 2,3 \pi 2,5 \pi 2, \ldots \ldots(n+1 / 2) . \pi$ and which agree with Wheel-Rim positions The Nucleus frequency is $w n=[\mathrm{n}+12] . \pi T . \sqrt{ } G \rho$ where, $\mathrm{G}=$ the shear modulus of Elasticity and,$\rho=$ the density of the line sector $\mathrm{T}$ equal to $\left[\varepsilon \mathrm{E}^{2}+\mu \mathrm{H}^{2}\right]$

In this way all the nucleus forces, Repulsions and Attractions are involved in holding a nucleus together .

\section{Hydrogen -Bonds}

1.. Hydrogen : The Quantum numbers are $\mathrm{n}=1, \mathrm{E}=1,1=\mathrm{n}-1=0 \quad m_{l}=21+1=-1 m_{l} \cdot \operatorname{Rim}, m_{s}=[-\downarrow] \neq 0$

2.. Helium : The Quantum numbers are $\mathrm{n}=2, \mathrm{E}=2,1=\mathrm{n}-1=1 \quad m_{l}=21+1=3 m_{l} \cdot \operatorname{Rim}, \boldsymbol{m}_{\boldsymbol{s}}=2[+\uparrow-\downarrow]=\mathbf{0}$

3.. Lithium : The Quantum numbers are $\mathrm{n}=3, \mathrm{E}=1,1=\mathrm{n}-1=2 \quad m_{l}=21+1=5 m_{l} \cdot \operatorname{Rim}, m_{s}=2[+\uparrow-\downarrow]+\uparrow \neq 0$

4.. Beryllium :The Quantum numbers are $\mathrm{n}=4, \mathrm{E}=2,1=\mathrm{n}-1=3 m_{l}=21+1=7 m_{l} \cdot \operatorname{Rim}, \boldsymbol{m}_{\boldsymbol{s}}=2[+\uparrow-\downarrow]+\uparrow-\downarrow=\mathbf{0}$

5.. Boron : The Quantum numbers are $\mathrm{n}=5, \mathrm{E}=3,1=\mathrm{n}-1=4 \quad m_{l}=21+1=9 m_{l} \cdot \operatorname{Rim}, m_{s}=2[+\uparrow-\downarrow]+\uparrow-\downarrow \neq 0$

6.. Carbon : The Quantum numbers are $\mathrm{n}=6, \mathrm{E}=4,1=\mathrm{n}-1=5 \quad m_{l}=21+1=11 m_{l} \cdot \operatorname{Rim}, \boldsymbol{m}_{s}=2[+\uparrow-\downarrow]+\uparrow-\downarrow=\mathbf{0}$

7.. Nitrogen : The Quantum numbers are $\mathrm{n}=7, \mathrm{E}=5,1=\mathrm{n}-1=6 \quad m_{l}=21+1=13 m_{l} \cdot \operatorname{Rim}, m_{s}=2[+\uparrow-\downarrow]+\uparrow-\downarrow \neq 0$

i.e. Helium is the First Stable, Plane, Stereo-Slate-Wheel-Rim, while Carbon is the First Stable, Space , three-dimensional structure Stereo-Slate-Wheel-Rim, and this is the why Hydrogen covalent Bonds with Carbon are the strongest that allows carbon to share its outer valence electrons with up to four Hydrogen .

The Obvious Inference is that the First-Gap can be filled with Neutrons Units only, $\varnothing$, While, The SecondGap can be filled with Negative Units only , $\Theta$, i.e. Two different containers in each Rim .Noble-atoms are exceptionally Stable therefore reflect their Electro-configuration.

1.. Hydrogen Atom Rotor:

It was shown that, the continuous Contact of opposite creates Circular Motion by following Newton`s Third law. This motion creates an Energy Wheel-Rim or a Cave in where Negative breakage, the Electron ,of radius $\mathbf{r}$, and mass $\mathbf{m}$, Rotates on the Positive breakage, the Nucleus, of the same radius $\mathbf{r}$. Inference are the two Gaps which can be filled with Neutrons Units only, $\varnothing$, while the Second-Gap can be filled with Negative Units only ,$\ominus$, and in atoms from Neutrons and Electrons , i.e. Two different containers in the same Gap.

Magnetic Spin of a single Hydrogen Electron is either $+1 / 2$ or $-1 / 2$ i.e. unstable, while for a Covalent bond is zero 
and this because each of the shared electrons belongs to both atoms in the filled shell . Figure 20 . Because of the Zero magnetic Spin, $\boldsymbol{m}_{\boldsymbol{s}}=0$ and in the absence of a Dipole-moment, C-H bonds is regarded as being nonpolar and this completes both of their outer shells making them stable. This logic in Particles follows, The First Law of Thermodynamics which states that, The Total energy of a System and its surroundings is constant, which is the same as, Energy can be neither created nor destroyed but can take different forms as above referred. Unzipping of DNA is a breaking of Hydrogen bonds which help hold the two strands of the double helix together. Kinetic-Energy is associated with the random motion of molecules while Potential-Energy is referred to the ability of Energy to be released on the occurrence of some process and by following the Euclidean logic . The one Electron is spinning because of Electrons mass , and SU = Static-Unbalance while the Wheel-Rim is Stable because of the rocking Moment and is referred to as DB = Dynamic-Balance.

\section{Helium Atom Rotor :}

Generally is holding most of these of Hydrogen . Nucleus has Two Protons and One Wheel-Rim which is filled with two Electrons of magnetic Spin $\boldsymbol{m}_{\boldsymbol{s}}=0$, and Zero Dipole-moment i.e. is the First Stable, Plane , Stereo-Slate-Wheel-Rim. Because of Two Protons and Electrons Wheel-Rim is greater, containing all Electrons of Two available Positions. Covalent bonds are zero because each Pair of the shared electrons in the over-filled-Shell is completed with another Pair in the outer shell making them Stable. The Two pairs of Electrons are inversely spinning and this of their mass in the Filling Rim, and exists $\mathbf{S B}=$ Static-Balance i.e. Stable, while the Wheel-Rim is Stable because of the rocking Moment and is referred to as DB = DynamicBalance .

\section{3.. Lithium Atom Rotor :}

Generally is holding most of these of prior . Nucleus has Three Protons and Two Wheel-Rims, One of which is filled with two Electrons of magnetic $\operatorname{Spin} \boldsymbol{m}_{\boldsymbol{s}}=0$, and Zero Dipole-moment while the Second Rim is of One Electron ( from the 8 available ) with Non-zero Dipole-moment, i.e. is Unstable . Because of the Three Protons and Electron, Wheel-Rim is greater, containing all Electrons available Positions . Covalent bonds are Not-zero because the Pair of the shared electrons in the Empty-Rim is not completed with another Pair in the Outer shell and to make them Stable i.e. exists $\mathbf{S U}=$ Static-Unbalance and $\mathbf{D U}=$ Dynamic-Unbalance because the One pair of Electrons is spinning additionally of their mass in the Empty Rim, while the Rotor is Stable because of the rocking Moment and is referred to as $\mathbf{D B}=$ Dynamic-Balance .

\section{4.. Beryllium Atom Rotor :}

Generally is holding most of these of prior . Nucleus has Four Protons and Two Wheel-Rims, the One of which is filled with two Electrons of magnetic Spin $\boldsymbol{m}_{\boldsymbol{s}}=0$, and Zero Dipole-moment while the Second Rim is of Two Electrons ( from the 8 available ) with Zero Dipole-moment, i.e. is Stable . Because of the Four Protons and the Two Electrons, Wheel-Rim is greater, containing all Electrons available Positions. The covalent bonds are Zero because the Pairs of the shared electrons in the Empty-Rim are completed with opposite Pairs in the Outer Non-filled-Shell and make them Stable, i.e. exists SB = Static-Balance and because the Two pairs of Electrons are spinning eccentrically with their mass in the Empty Rim, so exists DU = Dynamic Unbalance, while main Rotor is Stable because of the rocking Moment and is referred as DB = DynamicBalance .

\section{Boron Atom Rotor :}

Generally is holding most of these of prior . Nucleus has Five Protons and Two Wheel-Rims, the One of which is filled with two Electrons of magnetic Spin $\boldsymbol{m}_{\boldsymbol{s}}=0$, and Zero Dipole-moment while the Second Rim is of Three Electrons ( from the 8 available ) with Non-Zero Dipole-moment, i.e. is Un-stable . Because of the Five Protons and the three Electrons, Wheel-Rim is greater, containing all Electrons available Positions. The covalent bonds are Not-zero because the Pairs of the shared electrons in the Empty-Rim are not completed with opposite Pairs in the Outer Non-filled-Shell and to make them Stable, i.e. exists SU = Static-Unbalance and because the Three pairs of Electrons are spinning eccentrically with their mass in the Empty Rim, so exists DU $=$ Dynamic - Unbalance, while main Rotor is Stable because of the rocking Moment and is referred as $\mathbf{D B}=$ Dynamic-Balance . 


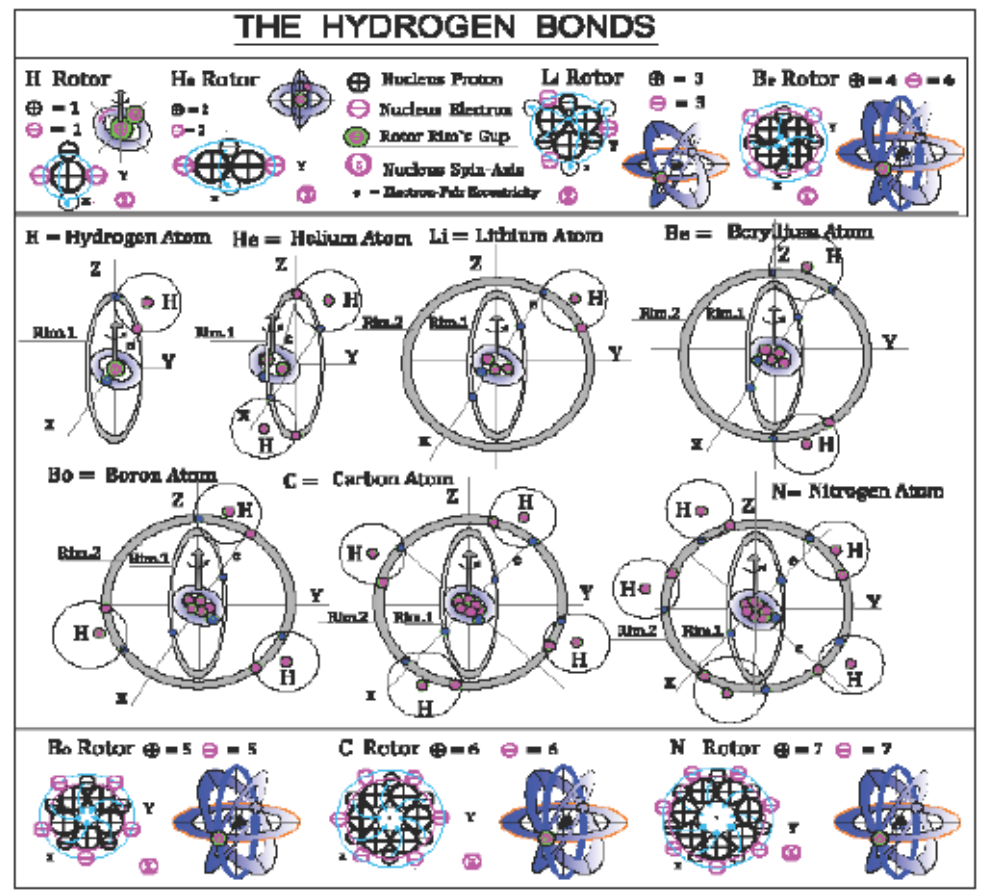

Figure. 25.

The Hydrogen-Atoms Bonds concerning Stability of Wheel-Rims and Protons in Nucleus. H to C Rotor -Electrons follow circular motion while $\mathbf{N}$ Rotor follows circular and Elliptical.

\section{6.. Carbon Atom Rotor :}

Generally is holding most of these of prior . Nucleus has Six Protons and Two Wheel-Rims, the One of which is filled with two Electrons of magnetic Spin $\boldsymbol{m}_{\boldsymbol{s}}=0$ and Zero Dipole-moment while the Second Rim is of Four Electrons ( from the 8 available ) with Zero Dipole-moment, i.e. is Stable. Because of the Six Protons and the Four Electrons, Wheel-Rim is greater, containing all Electrons available Positions . The covalent bonds are Zero because the Pairs of the shared electrons in the Empty-Rim are completed with opposite Pairs in the Outer Non-filled-Shell and make them Stable, i.e. exists SB = Static-Balance and because the Four pairs of Electrons are spinning symmetrically with their mass in the Empty Rim , so exists DB = Dynamic -Balance , while main Rotor is Stable because of the rocking Moment and is referred as

DB = Dynamic-Balance . i.e.

Carbon atom is the First Stable, Space, Stereo-Slate-Wheel-Rim occupying Static-Balance in Rim and Dynamic -Balance of the three axial Rims X, Y, Z and can Combine with Hydrogen forming CH4. This Unique property of Carbon allows to form a wide variety of Compounds, using any Chemical-Reaction. Since Atoms combine in certain fix ratios determined by the combining Power of atoms then in the Four available positions, the first Atom of Hydrogen forms $\mathrm{CH} 4$ which produces the first Unreactive Organic Compound and with other carbon atoms making Strong and Stable chains and rings .

\section{7.. Nitrogen Atom Rotor :}

Generally is holding most of these of prior . Nucleus has Seven Protons and Two Wheel-Rims, the One of which is filled with two Electrons of magnetic Spin $\boldsymbol{m}_{\boldsymbol{s}}=0$, and Zero Dipole-moment while the Second Rim is of Five Electrons ( from the 8 available ) with Non-Zero Dipole-moment, i.e. is Un-stable . Because of the Seven Protons and the Five Electrons, Wheel-Rim is greater, containing all Electrons available Positions. The covalent bonds are Not-zero because the Pairs of the shared electrons in the Empty-Rim are not completed with opposite Pairs in the Outer Non-filled-Shell and to make them Stable, i.e. exists $\mathbf{S U}=$ Static-Unbalance and because the Five pairs of Electrons are spinning eccentrically with their mass in the Empty Rim ,so exists $\mathbf{D U}=$ Dynamic -Unbalance, while main Rotor is Stable because of the rocking Moment and is referred as DB = Dynamic-Balance .

\section{8.. Atoms Rotor :}

For $\rightarrow \mathbf{m}=$ Electron mass $-\mathbf{M}=$ Rim`s Total mass $-\mathbf{e}=$ Eccentricity of Electrons Pair $-\mathbf{w}=$ Rotating angular velocity $-\mathbf{X}=$ The Resonant amplitude $-w_{n}=$ The Natural Electron-frequency $\boldsymbol{\zeta}=$ The Dumping factor Exists the Static and Dynamic Rotating Unbalance of Rims as below . 
Resonant Amplitude $\frac{\mathbf{M}}{\mathbf{m}} \frac{\mathbf{X}}{\mathbf{e}}=\frac{(\mathrm{w} / \mathrm{wn})^{2}}{\sqrt{\left(1-(\mathrm{w} / \mathrm{wn})^{2}\right)^{2}}+(2 \zeta \cdot \mathrm{w} / \mathrm{wn})^{2}}, \tan \boldsymbol{\varphi}=\frac{2 \zeta(\mathrm{w} / \mathrm{wn})}{1-(\mathrm{w} / \mathrm{wn})^{2}}$

The Steady-state Rotating Unbalancing •

Resonant Amplitude AO $=$ e $\cdot\left(\frac{w}{w n}\right)^{2}: \sqrt{\left[1-\left(\frac{w}{w n}\right)^{2}\right]^{2}+\left[2 \zeta\left(\frac{w}{w n}\right)\right]^{2}}$

A Synchronous Whirling Rotating Shaft .

(a).. Is the equation of motion of a Harmonic disturbing force resulting from rotating Unbalance . (b).. Is the equation of motion of Synchronous whirl indicating that ,e, leads Resonant amplitude by the phase angle,$\varphi$, depending on damping $\zeta$, and rotation speed ratio w/wn. When w/wn coincides with the critical speed $w n=\sqrt{\mathrm{k} / \mathrm{m}}$ the amplitude is restrained only by the damping.

\subsection{Why , Glue-Bond Electrons of Nucleus Rotor , form Rhodonea -like-Curves ?}

Curves created by Rotor`s electron are such that they keep a continuous pressure on Protons as in Fig-26.

1.Continuous action of Negative $\Theta$, on One Positive $\bigoplus$, is equivalent to a Circular motion of $\ominus$ to $\bigoplus$, with velocity $\mathrm{v}=\sqrt{\mathrm{F}} \mathrm{r} / \mathrm{m}$. The circular motion is because $\mathrm{l}=\mathrm{r}=$ constant.

2. Continuous action of Negative $\ominus$, on Two Positive $\bigoplus \bigoplus$ of glued bond, is equivalent to a Circular - and Other of one-step longer motion of $\ominus$ to $\bigoplus \bigoplus$, or Circular motion on the Common-length . Length , 1 , in polar equations is, $\mathbf{l}=\mathbf{a} \cdot \cos (\mathbf{c} \cdot \boldsymbol{\theta})$ or $\mathbf{l}=\mathbf{a} \cdot \sin (\mathbf{c} \cdot \boldsymbol{\theta})$ where $\mathbf{a}=$ the length of the base $=2 \mathrm{r}+2 \mathrm{r}=4 \mathrm{r}$, $\mathbf{c}=$ Rim`s number which produce a c-petaled rose, if $\mathbf{c}$ is odd or 2c-petaled rose and if $\mathbf{c}$ is even number. The period of both $\cos (c \theta), \sin (c \theta)$ is $2 \pi / c$, and the number of petals for the period is $[0,2 \pi / c]$ will be $\mathrm{c}$ or $2 \mathrm{c}$, according to as $\mathrm{c}$ is odd or $\mathrm{c}$ is even, so the degree of the Cartesian equation of the curve is , $\mathrm{c}+1$ for odd-c , and 2(c+1) for even-c .

3. Continuous action of Negative $\ominus$, on Three Positive $\bigoplus \bigoplus \bigoplus$ of glued bond, is equivalent to a Circular-and Other of two-step longer motion of $\ominus$ to $\bigoplus \bigoplus \bigoplus$, or Circular motion on the Common-length . Length, 1, in polar equations is, $\mathbf{l}=\mathbf{a} \cdot \cos (\mathbf{c} \cdot \boldsymbol{\theta})$ or $\mathbf{l}=\mathbf{a} \cdot \sin (\mathbf{c} \cdot \boldsymbol{\theta})$ where $\mathbf{a}=$ the length of the base $=2 r+2 r+2 r=6 r$,

$\mathbf{c}=$ Rim`s number which produce a c-petaled rose , where $\mathbf{c}$ is odd or $2 \mathrm{c}$-petaled rose number.

This is seen and from equations issuing in Figure.22-2 $\rightarrow r=r D \cdot \sin \varphi$ and $R=R D \cdot \sin \varphi$ where,$a$, are the equal radius of point $\mathrm{A}$ of polar path .

Rhodonea curves follow the Odd or and Even number of Protons in Nucleus by changing Rolling-length $\mathrm{a}=(2 \mathrm{r})$ , and the Polar - length $1=\mathrm{a} \cdot \cos (\mathrm{c} \cdot \theta)$ depending on,$\theta$, as above. The common bonding point of the Negative circle and the Positive line of the rolling circle obliges that point to follow the cycloid motion in the maximum degree and thus executing Hypocycloid motion .

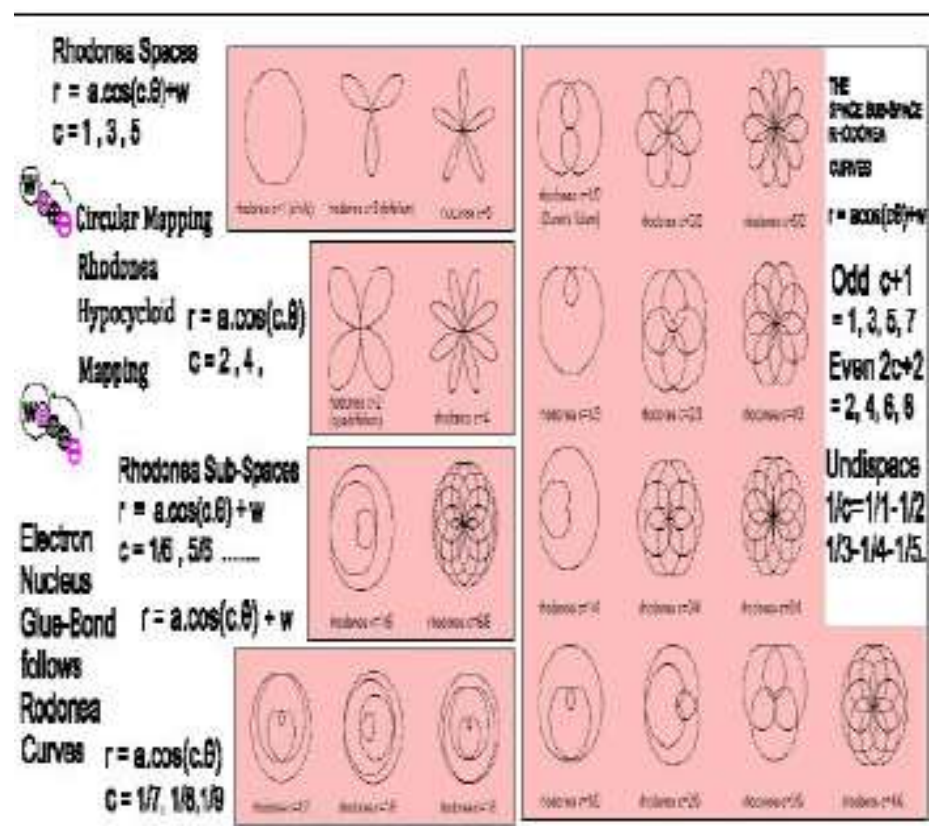

Figure. 26.

The Rhodonea Curves Configuration where $\mathrm{a}=$ the length of the petals of the rose , $\mathrm{c}=$ Number of Protons in Rims . Rotor`s Electrons follow circular motion while , 
c.th Rotor follows Rhodonea Hypocycloid curves.

7.10. Epilogue .

The origin of Space [S] becomes, through the Principle of Virtual Displacements $\mathrm{W}=\int \square . \square \square \square \square=0$, from Primary Point A which is the Space, to B which is the Anti-space as the Inner distance of Space and Anti-Space in all Layers .

1) The origin of Energy becomes, through the Principle of Virtual Displacements, as the Work of the Inner Impulse distance of Space and Anti-Space embedded in all points of universe.

2) The minimum Quantized Space, Quanta, $\mathrm{s}^{2}$, are in all quantized Spaces, i.e. Particles , [MFMF] Field , moving vectors, free velocity monads, Material Points and lines $\rightarrow$ Surfaces and bodies.

3) The minimum Quantized Energy, Quanta $2 \mathrm{~s}^{2}$, is diffused as energy, SPIN ,in all quantized spaces as material volumes.

4) The definition of Material Point as $( \pm)$ Dipole Breakage in [MFMF] medium.

5) The definition of Material Object as the Wavelength,$\lambda$, of the $( \pm)$ Dipole of $[$ MFMF] Field which is a Standing Electromagnetic wave in tiny energy volumes and exists in beyond Planck`s cavity.

6) The Time in Euclidean geometry is not distinguished, because time is a conversion factor, a relative measure of changes, existing only in its confined-Plank's length level because there exists motion -and is neither Space from Energy -because Energy exists as quanta on any first dimensional Unit $A B$ with infinite or zero time which connects the only two fundamental elements of Universe, that of Points and that of Energy. Time is designated as the meter of changes, or as the conversion factor, between time (second) and space (mass) units and not essence of Space-energy Configuration. This age-old question, of what is time, was standing for many centuries in Philosophy, and was recently adapted by GR, by considering Time, as an essence of Space-time and accepting as base the wrong Non-

Euclidean geometries.

7) The [STPL] line-cylinder as the passage of energy particles from Absolute [S] Frame to all Relative [R] Frames without any diffusion which consists the Navel cord, the string, of galaxies.

8) The geometrical Reasoning of Planck Length, Gravity`s length, and all Spaces length caves .

9) Work as Energy is Quantized, converted, in Space monads, caves, $\bar{x}=\overline{d s}=\lambda \mathrm{m}$, as pressure $\sigma, T$, the pressure is converted in caves as a Standing Electromagnetic Wave [E,P] which consists the Standing monad ( Maxwell's Displacement current ) and the moving Energy monad ( by altering the inner wavelength $\lambda$ or Period $\mathrm{T}$ of monad ) in Gravity`s field medium [MFMF] and is dissipated as Quaternion monads ( Particles or Waves, matter or vectors) as Forces (displacements, masses, pressure etc.) using modulus, coefficients, reactions to the motion and all other geometrical indices as prior referred.

10) The Geometries related to the Euclidean by Lorentz factor,,$\gamma$, consist, The Geometrical expression of Spaces and Anti-Spaces in Relative systems and is represented by STPL cylinder lines .

11) The Cause and Events as it is the Energy quantization in caves $\lambda$ and the velocity Breakages as , masses , and velocity, $\overline{\mathrm{v}}$, only is the Thrust of masses.

12) The Origin and the base of Gravity from $[\mathrm{S}]$ to $[\mathrm{R}]$ frame, and the Equations of the inner standing Electromagnetic wave.

13) The Structure of the Energy -Space Universe and the boundaries of the preceding Space-time of General Relativity.

14) The Origin of Particles from velocity -The Thrust -on Breakages and the Breakages as the Fragments of the Space, Anti-Space collision, of a cave.

15) The Origin of Color -forces from the Retardation and the Birefringence of Spaces .

16) The Dual nature of Monads, of wavelength $|\lambda|$ as Particle , and Wave as the Stationary Electromagnetic Wave in $|\lambda|$, which is an intrinsic property of the Cycloid motion of velocity , $\bar{c}$, and Spin, forming the plane and spherical standing waves which are < The Inner structure of Particles >.

17) The Cycloidal motion as the intrinsic property of vectors which is a Stationary wave on wavelength of vector, where < Poinsot's ellipsoid > becomes $\rightarrow$ cycloid Ellipsoid and spin to be $1 / 2$ and 1 ,

18) The mechanical relation between the speed of light and principal stresses , guided to, Permittivity , Permeability, in free space medium.

19) Michelson`s-Morley experiments cannot prove reality because in Planck`s cave occurs isochrones motions , and Gravity is the force (energy) which is connecting the Material points of the Medium with the constant light velocity $\bar{c}$.

20) The Relative motion of moving systems through Stationary system can be detected only by the Tangential velocities $\bar{v}=\bar{w} . r$ on circumference of a cave,$r$, with radius $R>r$.

21) The equations of inner Structure of Gravity field and the stability of the whirling Space, Anti-space, the Spin and Momentum, are extended to moving monads. 
22) The immense confusion in the basic ideas regarding the Quantized Energy-Space, what are The Energy and Space Quanta , $\rightarrow$ vanishes.

23) The Manifold of mathematics from Astrophysics to Quantum mechanics have been progressively developed on Non-Euclid Geometries, resulting to Relativity`s Space-time confinement, unable to conceive the under Planck`s cavity energy existence. The only two fundamental elements of Universe, is that of Space ( points ) and that of Work = Energy (motion) and is the only way to explain rightly the outside Reality .

24) In articles was presented the Creation Hypothesis without Big-Bang and without any other coherent Energylevel. An extend elucidation has been previously done for some very important definitions as that of Quanta of Space and the Quanta of Energy. The expansion of the universe occurs by THE rolling, of the light velocity Moving Dark-Matter -Energy Heap mixture, on the Rest Gravity-Field-Energy Base, which consists a parallel motion and not parallel universes as said. It has been elucidated the Origin and the Nature of Particles Fermions and Bosons, what is Gravity -field , Gravity -force , Dark -matter and Dark-energy . It was analyzed that Gravity-field is a Rest of two opposite signed elements which consist the minimum energy quanta Spinning space and this because of dipole nature, and jointed by the Gravity-force which is the Maxwell`s-Gravity`s Displacement current, and which acts on any other moving or not particle . Because Dark-matter moves with the constant velocity ,c, and is composed of the two opposite signed elements also, and Dark-energy moves also with velocity of light, so is continually effecting on the two fragments separately and are slinging them further , formulating the Infinite and attracting Geometrical Universe , i.e. Thrust $[\overline{\mathrm{c}}$. $\square \mathrm{i}]$ with light velocity , $\overline{\mathrm{c}}$, is acting on the Five Energy and Space Fragments $\rightarrow$

$\left\{(\square \mathrm{i}),\left(+\mathrm{s}^{2}\right),\left(-\mathrm{s}^{2}\right),\left(+\mathrm{cs}^{2}\right),\left(-\mathrm{cs}^{2}\right)\right\}$ and on $\rightarrow\left[(\square \mathrm{i}) \rightarrow\left(+\mathrm{s}^{2}\right) \leftrightarrow\left(-\mathrm{s}^{2}\right)\right]=$ The Energy-dipole Gravity-field $]$ and also on $\rightarrow\left[(\square \mathrm{i}) \rightarrow\left(+\mathrm{cs}^{2}\right) \leftrightarrow\left(-\mathrm{cs}^{2}\right)=\right.$ The Energy-dipole Gravity-Dark-matter -field $]$ carrying them in the three dimensional parallel space with the light velocity , $\bar{c}$, as the Rest Gravity -field -Energy , and the rolling Movable Heap on it, the Dark-matter-energy, or the mixture of the spherical and opposite signed elements and which highlight, and the dipole from them jointed both by the Gravity force, which form the heavy and massive invisible Dark matter, the dipole energy blobby volumes which are the massive Dark Fringes which repel . This is the Expanding universe without Big-Bang, based on Euclidean Geometrical logic only, which is the outer reality of it, and not on Axioms and Postulates which do not agree with this Physical reality.

25) In article is shown the How Positive (+) and Negative (-) are joint to create material point which is Zero and by adding it to monads, to create all Primary Particles, following Extrema Geometry-Spaces-Moulds , which are the in Periodic Table elements in each Space-Level . It was shown also the How Rest-Gravity constituent MFMF field is joint and creates the Electromagnetic Waves E,P and Gravity-Spin, which External Spin , is continually converted to the Inner Electromagnetic Waves E,P and which continue this eternal cycle.

26) In summary, my personal confidence is that nature is produced from Euclidean Geometry only .\{ i.e. $\rightarrow$ from Primary Point A which is nothing ( the non-existence ) by becoming Anti-point B ( the opposite to nonexistence also $)$ as Segment $\mathrm{AB},($ discrete $=$ existence $=$ monad $)$, which Equilibrium by opposite forces following Virtual work and thus being the Quaternion ( the monad $\mathrm{AB}$ as Space $\mathrm{AB}$ and as energy equal to Virtual work ) and consisting the Primary Neutral Space .

1.. Equilibrium on a Straight line , of a Rolling -Particle is assured by Cycloidal -Evolute motion,representing the Cycloid $=$ The - Space and Evolute $=$ The Anti-space, and happens to Fundamental moving - Particles .

2.. Equilibrium on a Circle, of a Rolling -Particle, a breakage, is assured by the Wheel-Rims caves motion , epresenting the Nucleus $=$ The - Space and Orbitals $=$ The Anti-space, and happens to Atoms and Particles .

3.. Equilibrium In-Out wavelength is obtained by the Isochronous Anti-Standing Electromagnetic

Wave E,P which happens on Anti-cycloid, Evolute, and whirling, Curl , by the equality of the two transverse Complex Envelope displacements, Amplitudes, that consist the Standing monad as

(Coriolis force curl helix Displacement current in monads), or

4.. Orbital Equilibrium is obtained by the Circular glue Motion of the Negative on Positive, where is created an Energy Wheel-Rim or a Cave, in where Negative breakage the Electron, of radius $r$ and mass $m$ is rolling on Cave's Wall, and because executes a pressure then is positive .

5.. Stability of the Nucleus Dynamic System is maintained by the Work produced by the Rotor on to RhodoneaWheel-Rims as Torsional momentum .

Orbital Equilibrium is generally obtained by the Circular glue Motion of the Negative on Positive , where is The accumulation of disarrangement of Sub -Spaces leads to a powerful vortex from the infinite small vortices, which by entering in Geometry Caves, and through the most important Geometrical mould [the STPL cylinder] on cave, where there $\rightarrow$ Spaces (A), Anti-spaces (B) and Sub-spaces (AB) co-exist on a common circle of diameter $\mathrm{AB}$, and by the composition of opposites ( it is the dipole $\rightarrow[+\leftrightarrow-]$ ), through GSSMMechanism produce through the quantization of energy to quantization of Spaces , to Particles, to Gravityfield-forces , to Dark-matter-gravity forces, following Geometry principles, which become thus Physical , and not from any other tile-guided center or logical starting point . 


\section{References}

[1]. Matrix Structure of Analysis by J.L.MEEK library of Congress Catalog 1971

[2]. Der Zweck im Rect by Rudolf V. Jhering 1935

[3]. The great text of J. L.Heisenberg (1883-1886) and the English translation by Richard Fitzpatrick. Elements Book 1.

[4]. Wikipedia.org, the free Encyclopedia.

[5]. Greek Mathematics, Sir Thomas L.Heath -Dover Publications, Inc, New York. 63-3571.

[6]. [T] Theory of Vibrations by William T. Thomson (Fourth edition).

[7]. A Simplified Approach of Squaring the circle, http://www.scribd.com/mobile/doc/33887739

[8]. The Parallel Postulate is depended on the other axioms, http://vixra.org/abs/1103.0042

[9]. Measuring Regular Polygons and Heptagon in a circle, http://www.scribd.com/mobile/doc/33887268

[10]. The Trisection of any angle ,http://vixra.org/abs/1103.0119

[11]. The Euclidean philosophy of Universe, http://vixra.org/abs/1103.0043

[12]. Universe originated not with BIG BANG, http://www.vixra.org/pdf/1310.0146v1.pdf

[13]. Complex numbers Quantum mechanics spring from Euclidean Universe,

[14]. http://www.scribd.com/mobile/doc/57533734

[15]. Zeno`s Paradox, nature of points in quantized Euclidean geometry,

[16]. http://www.scribd.com/mobile/doc/59304295

[17]. The decreasing tunnel, by Pr. Florentine Smarandashe, http://vixra.org/abs/111201.0047

[18]. The Six-Triple concurrency line -points, http://vixra.org/abs/1203.0006

[19]. Energy laws follow Euclidean Moulds, http://vixra.org/abs/1203.006

[20]. Higgs particle and Euclidean geometry, http://www.scribd.com/mobile/doc/105109978

[21]. Higgs Boson and Euclidean geometry, http://vixra.org/abs/1209.0081

[22]. The outside relativity space - energy universe, http://www.scribd.com/mobile/doc/223253928

[23]. Quantization of Points and of Energy, http://www.vixra.org/pdf/1303.015v21.pdf

[24]. Quantization of Points and Energy on Dipole Vectors and on Spin, http://www.vixra.org/abs/1303.0152

[25]. Quaternion`s, Spaces and the Parallel Postulate, http://www.vixra.org/abs/1310.0146

[26]. Gravity as the Intrinsic Vorticity of Points, http://www.vixra.org/abs/1401.0062

[27]. The Beyond Gravity Forced fields, http://www.scribd.com/mobile/doc/203167317

[28]. The Wave nature of the geometry dipole, http://www.vixra.org/abs/1404.0023

[29]. The Outside Relativity Space-Energy Universe, http://www.scribd.com/mobile/doc/223253928

[30]. Planks Length as Geometrical Exponential of Spaces, http://www.vixra.org/abs/1406.0063

[31]. Universe is built only from Geometry Dipole, Scribd: http://www.scribd.com/mobile/doc/122970530

[32]. Gravity and Planck`s Length as the Exponential Geometry Base of Spaces, http://vixra.org/abs/1406.0063

[33]. The Parallel Postulate and Spaces ( IN SciEP )

[34]. The Origin of the fundamental particles in Planck`s Confinement. On Scribd \& Vixra (FUNDAPAR.doc)

[35]. The fundamental particles of Planck`s Confinement. www.ijesi.com (IJPST14-082601)

[36]. The origin of The fundamental particles www.ethanpublishing.com(IJPST-E140620-01)

[37]. The nature of fundamental particles, (Fundapa.doc).www.ijesit.com-Paper ID:IJESIT ID: 1491

[38]. The Energy-Space Universe and Relativity IJISM, www.ijism.org-Paper ID: IJISM -294

[39]. [V2,I6,2347-9051]

[40]. The Parallel Postulate, the other four and Relativity (American Journal of modern Physics, Science PG -

[41]. Publication group USA), 1800978 paper

[42]. Space-time OR, Space-Energy Universe ( American Journal of modern Physics, science PG Publication group USA ) 1221001Paper.

[43]. The Origin of ,Maxwell`s-Gravity`s, Displacement current . GJSFR (Journalofscience.org), Volume 15-A, Issue 3 , Version 1.0

[44]. Young`s double slit experiment [ Vixra: 1505.0105] Scribd : https://www.scribd.com/doc/265195121/

[45]. The Creation Hypothesis of Nature without Big-Bang. Scribd : ttps://www.scribd.com/doc/267917624 /

[46]. The Expanding Universe without Big-Bang . (American Journal of modern Physics and Applications

[47]. Special issue: http://www.sciencepublishinggroup.com/j/Science PG-Publication group USA -622012001-Paper.

[48]. The Parallel Postulate and the other four, The Doubling of the Cube ,The Special problems and Relativity. https://www.lappublishing.com/. E-book. LAMBERT Academic Publication .

[49]. The Moulds for E-Geometry Quantization and Relativity , International Journal of Advances of

[50]. Innovative Research in Science Engineering and Technology IJIRSET

[51]. http://www.ijirset.com/..Markos Georgallides

[52]. [M] The Special Problems of E-geometry and Relativity http://viXra.org/abs/1510.0328

[53]. [M] The Ancient Greek Special Problems as the Quantization Moulds of Spaces. www.submission.arpweb.com(ID-44031-SR-015.0

[54]. [M] The Quantization of E-geometry as Energy monads and the Unification of Space and Energy .

[55]. Www.ijera.com(ID-512080.0

[56]. [51] The Why Intrinsic SPIN (Angular Momentum ) 1/2 -1 ,Into Particles .

[57]. www.oalib.com(ID-1102480.0

[58]. [M] The Kinematic Geometrical solution of the Unsolved ancient - Greek Problems and their Physical nature

[59]. http:www.jiaats.com/paper/3068.ISO 9001

[60]. [M] The Nature of Geometry the Unsolved Ancient-Greek Problems and their Geometrical solution .

[61]. www.oalib.com(paper. ID-1102605.0 http:www.oalib.com/Journal:paper/1102605

[62]. E-Geometry, Mechanics-Physics and Relativity, http:gpcpublishing.com/GPC : volume 4, number 2

\section{Journal Homepage}

[63]. [M] Material-Geometry and The Elements of the Periodic-Table .

[64]. www.ijerm.com(ID-0306031.0)

[65]. [M] The Material-Geometry Periodic Table of Particles and Chemistry . ??

[66]. [M] Material-Geometry and Physics.

[67]. [M] The origin of Black-holes and Black-matter . 
[68]. [M] The origin of SPIN of the fundamental Particles and their Eternal motion.

[69]. [M] The Doubling of the Cube .

[70]. The Squaring of the circle.

[71]. [M] The origin of, Maxwell`s Postulates.

[72]. $\quad[\mathrm{M}]$ The Quantization of Points and Potential and the Unification of Space and Energy with the

[73]. universal principle of Virtual work, on Geometry Primary dipole.

[74]. by Markos Georgallides.

Markos Georgallides comes from Cyprus and currently resides in the city of Larnaca, after being expelled from his home town Famagusta by the Barbaric Turks in August 1974. He works as a consultant civil and architect engineer having his own business. He is also the author of numerous scholarly articles focusing on Euclidean Geometry and mathematical to physics related subjects. He obtained his degree from the Athens, National Technical, Polytechnic University [NATUA] and subsequently studied in Germany, Math theory of Photoelasticity. 